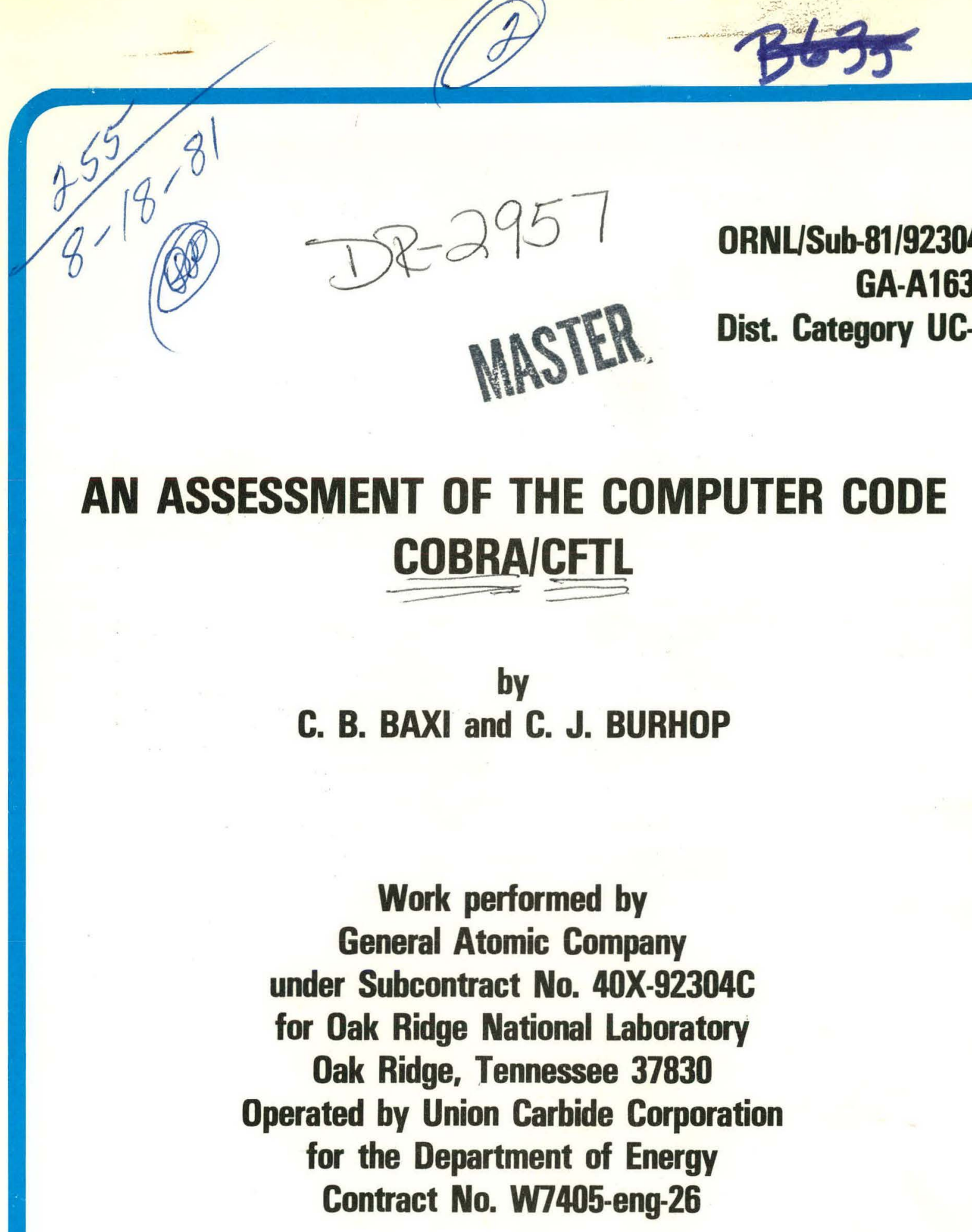

\title{
AN ASSESSMENT OF THE COMPUTER CODE COBRA/CFTL
}

\author{
by
}

C. B. BAXI and C. J. BURHOP

Work performed by

General Atomic Company

under Subcontract No. 40X-92304C

for Oak Ridge National Laboratory

Oak Ridge, Tennessee 37830

for the Department of Energy

Contract No. W7405-eng-26

JULY 1981 


\section{DISCLAIMER}

This report was prepared as an account of work sponsored by an agency of the United States Government. Neither the United States Government nor any agency Thereof, nor any of their employees, makes any warranty, express or implied, or assumes any legal liability or responsibility for the accuracy, completeness, or usefulness of any information, apparatus, product, or process disclosed, or represents that its use would not infringe privately owned rights. Reference herein to any specific commercial product, process, or service by trade name, trademark, manufacturer, or otherwise does not necessarily constitute or imply its endorsement, recommendation, or favoring by the United States Government or any agency thereof. The views and opinions of authors expressed herein do not necessarily state or reflect those of the United States Government or any agency thereof. 


\section{DISCLAIMER}

Portions of this document may be illegible in electronic image products. Images are produced from the best available original document. 
Printed in the United States of America. Available from National Technical Information Service

U.S. Department of Commerce

5285 Port Royal Road, Springfield, Virginia 22161

NTIS price codes-Printed Copy: A011; Microfiche: A01

\begin{abstract}
This report was prepared as an account of work sponsored by an agency of the United States Government. Neither the United States Government nor any agency thereof, nor any of their employees, makes any warranty, express or implied, or assumes any legal liability or responsibility for the accuracy, completeness, or usefulness of any information, apparatus, product, or process disclosed, or represents that its use would not infringe privately owned rights. Reference herein to any specific commercial product, process, or service by trade name, trademark, manufacturer, or otherwise, does not necessarily constitute or imply its endorsement, recommendation, or favoring by the United States Government or any agency thereof. The views and opinions of authors expressed herein do not necessarily state or reflect those of the United States Government or any agency thereof.
\end{abstract}




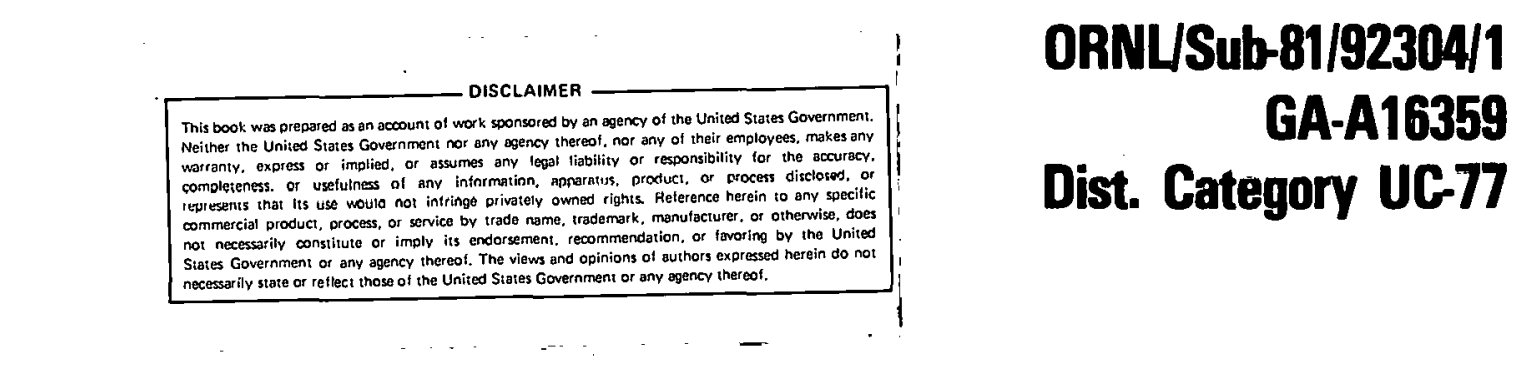

\section{AN ASSESSMENT OF THE COMPUTER CODE COBRA/CFTL}

by

C. B. BAXI and C. J. BURHOP

Work performed by

General Atomic Company under Subcontract No. 40X-92304C for Oak Ridge National Laboratory

Oak Ridge, Tennessee 37830 Operated by Union Carbide Corporation for the Department of Energy

Contract No. W7405-eng-26 JULY 1981 
THIS PAGE

\section{WAS INTENTIONALLY \\ LEFT BLANK}




\section{ABSTRACT}

The COBRA/CFTL code has been developed by Oak Ridge National Laboratory (ORNL) for thermal-hydraulic analys is of simulated gas-cooled fast breeder reactor (GCFR) core assemblies to be tested in the core flow test loop (CFTL). The COBRA/CFTL code was obtained by modifying the General Atomic code COBRA*GCFR.

This report discusses these modifications, compares the two code results for three cases which represent conditions from fully rough turbulent flow to laminar flow. Case 1 represented fully rough turbulent flow in the bundle. For this case, the agreement between the two code results for the pressure drop and coolant temperature calculations was within $1 \%$. The agreement for the interior channel rod temperatures was within $3 \%$. However, the wall channel rod and shroud temperatures differed by up to $8.5 \%$ and $23 \%$, respectively. Cases 2 and 3 represented laminar and transition flow regimes. For these cases, the agreement between the pressure drop and coolant temperature calculations was within 5\%. However, the rod surface temperatures varied by as much as $25 \%$ due to different spacer enhancement correlations and a different definition of start of heating location in the two cases. The reasons for the differences are discussed in detail in the report.

The required input for the COBRA/CFTL code, a sample problem input/ output and the code listing are included in the Apperidices. 
THIS PAGE

\section{WAS INTENTIONALLY \\ LEFT BLANK}




\section{NOMENCLATURE}

$A D$

$\mathrm{AH}$

AR

$C G(1)$ to $C G(7)$

CR(1) to $\operatorname{CR}(8)$

$\mathrm{D}_{\mathrm{H}}$

$f$

$G\left(h^{+}\right)$

$\mathrm{h}^{+}$

$\mathrm{h}_{\mathrm{R}}$

$\mathrm{K}$

$K(\operatorname{Re})$

m

$\mathrm{Nu}$

$\mathrm{Nu} u^{*}$

$\operatorname{Pr}$

Re

$R\left(h^{+}\right)$

$r_{0}$

$r_{1}$

$r$ function defined by Eqs. 19 and 21

slope of the temperature profile, Eq. 2

slope of the velocity profile, Eq. 1

constants, Eqs. 9 through 11

constants, Eqs. 3 through 6

hydraulic diameter

friction factor

function, Eq. 2

roughness cavity Reynolds number

rib height

function defined by Eqs. 24 and 26

function defined by Eq. 18

exponent, Eqs. 23 and 25

Nusselt number

multiplier on the Nusselt number due to spacer effect, Eqs. 16 and 22

Prandtl number'

Reynolds number

function, Eq. 1

radius of zero shear

volumetric radius of rough rod

radius of equivalent annulus 


$\begin{array}{ll}\mathrm{SH}_{1} & \begin{array}{l}\text { spacer length } \\ \text { Stanton number for the inner zone or } \\ \text { interior channel }\end{array} \\ \mathrm{St}_{1} & \begin{array}{l}\text { temperature } \\ \text { nondimensional temperature }\end{array} \\ \mathrm{t}^{+} & \text {nondimensional velocity } \\ \mathrm{u}^{+} & \text {axial distance } \\ \mathrm{Y}_{\mathrm{L}} & \text { radial distance } \\ \mathrm{Y}_{\mathrm{L}} & \text { length of the velocity profile } \\ \alpha & \text { radius ratio } \\ \varepsilon & \begin{array}{l}\text { ratio of spacer area to free flow area } \\ \text { in a channel }\end{array}\end{array}$

Subscripts

B

W

MAX

1 bulk temperature

wa11 temperature

maximum value

inner zone or interior channel 


\section{CONTENTS}

\section{$\underline{\text { Page }}$}

ABSTRACT

NOMENCLATURE

1. INTRODUCTION

2. MODIFICATIONS , 2

2.1 Removal of Unnecessary Routines 2

2.2 Modified $\mathrm{R}\left(\mathrm{h}^{+}\right)$and $\mathrm{G}\left(\mathrm{h}^{+}\right)$Functions 2

2.3 Stanton Number in. Interior Channels . 3

2.4 Variable Slopes AR and AH 4

2.5 SI (Metric) Units 4

2.6 Laminar Nusselt Numbers 1

2.7 Shroud Temperatures During Laminar Flow 5

2.8 Effect of Spacers on Heat Transfer During 5 Laminar and Transition Flow.

2.9 Input/Output Changes $\quad 7$

2.10 Step Change in Heat Flux 7

2.11 Correction for Smooth Geometry 7

2.12 Property Table Range 8

2.13 Test for Laminar Friction Factor 8

2.14 Imposition of Laminar Flow Calculations 8

2.15 Correction for Axial Flux when Rod Conduction 8 Calculations are Done

2.16 Correction to Bundle Averaged Results 8

2.17 Symmetry Option 8

2.18 Removal of Unnecessary Options · 9

3. CODE COMPARISONS 10

3.1 Test 1

3.2 Test 2 
3.3 Test 3

3.4 Summary of Code Comparisons

4. COST COMPARISONS

12

5: SUMMARY AND CONCLUSIONS

13

6. REFERENCES

15

APPENDIX A: INPUT CARD DATA DESCRIPTION

A-1

APPENDIX B: SAMPLE INPUT

B-1

APPENDIX C: SAMPLE OUTPUT

C-1

APPENDIX D: COBRA/CFTL CODE LISTING

D-1 


\section{FIGURES}

1. Subchannel and Rod Identification Numbers for the Test Bundie.

2. Comparison of COBRA*GCFR and COBRA/CFTL Results - Test 1: Pressure Drop.

3. Comparison of COBRA*GCFR and COBRA/CFTL Results - Test 1: Conlant and Rod Surface Temperatures in Interior Channel.

4. Comparison of COBRA $\star G C F R$ and COBRA/CFTL Results - Test 1: Cooland and Rod Surface Temperatures in Wall Channel.

5. Comparison of COBRA*GCFR and COBRA/CFTL Results - Test 1: Shroud Temperatures in Wall Channel.

6. Comparison of COBRA $\star G C F R$ and COBRA/CFTL Results - Test 1: Coolant and Rod Surface Temperatures in Corner Channel.

7. Comparison of COBRA $\star G C F R$ and COBRA/CFTL Results - Test 1: Coolant Temperatures in Channel Next to Hanger Rod.

8. Comparison of COBRA*GCFR and COBRA/CFTL Results - Test 2: Pressure Drop.

9. Comparison of COBRA*GCFR and COBRA/CFTL Results - Test 2: Coolant and Rod Surface Temperatures in Interior Channel.

10. Comparison of COBRA*GCFR and COBRA/CFTL Result.s - Test 2: Coolant and Kod Surface Temperatures in Wall Channel.

11. Comparison of COBRA*GCFR and COBRA/CFTL Results - Test 2:- Shroud Temperatures in Wall Channel.

12. Comparison of COBRA*GCFR and COBRA/CFTL Results - Test 2: Coolant and Rod Surface Temperatures in Corner Channel.

13. Comparison of COBRA*GCFR and COBRA/CFTL Results - Test 2: Coolant Temperatures in Channel Next to Hanger Rod.

14. Comparison of COBRA*GCFR and COBRA/CFTL Results - Test 3: Pressure Drop.

15. Comparison of COBRA*GCFR and COBRA/CFTL Results - Test 3: Coolant and Rod Surface Temperatures in Interior Channel. 
16. Comparison of COBRA*GCFR and COBRA/CFTL Results - Test 3: Coolant and Rod Surface Temperatures in Wall Channel.

17. Comparison of COBRA $\star G C F R$ and COBRA/CFTL Results - Test 3: Shroud Temperatures in Wall Channel.

18. Comparison of COBRA ${ }^{\star} G C F R$ and COBRA/CFTL Results - Test 3: Coolant and Rod Surface Temperatures in Corner Channel.

19. Comparison of COBRA*GCFR and COBRA/CFTL Results - Test 3: Coolant Temperatures in Channel Next to Hanger Rod. 


\section{TABLES}

1. Subroutines Removed From and Added to the COBRA*GCFR Code to Obtain the COBRA/CFTL Code.

2. Test Conditions for Code Comparisons.

3. Summary of Code Comparison.

4. Cost Comparison. 


\section{PAGES xii to xiii WERE INTENTIONALLY LEFT BLANK}




\section{INTRODUCTION}

The COBRA/CFTL code was developed by the Oak Ridge National Laboratory (ORNL) for thermal-hydraulic analysis of gas-cooled fast breeder reactor (GCFR) simulated core assemblies to be tested in the core flow test loop (CFTL) (Ref. 1) under construction at ORNL. The COBRA/CFTL code is essentially a modified version of the COBRA*GCFR code (Ref. 2) developed by General Atomic Company (GA). Thesc modifications (1) simplify the code for specific application to CFTL test bundles, (2) incorporate a new correlation method developed for rough surfaces by ORNL (Refs. 3 through $5)$, (3) incorporate new thermal-hydraulic correlations, (4) convert the code to metric units, and (5) incorporate new input and output formats. This report describes only these modifications to the COBRA*GCFR code. The report is intended as a supplement to Ref. 2. Together, these two reports provide a complete description and user's manual for the COBRA/CFTL code.

To evaluate the effect of the code modifications, calculation results of the COBRA/CFTL and COBRA*GCFR codes are compared with each other for three problems that cover all flow regimes. This report discusses these comparisons.

A set of input instructions, a sample input and output, and the listing for the COBRA/CFTL code are included in the Appendices. 


\section{MODIFICATIONS}

This section describes modifications made to the COBRA*GCFR code to obtain the COBRA/CFTL code. These changes were made to obtain a computer code that was simpler for specific application to thermal-hydraulic analys is of test bundles in the CFTL (Ref. 1). In addition, some changes were made to include correlations that were not available when development of the COBRA*GCFR code was stopped in February 1980.

\subsection{REMOVAL OF UNNECESSARY ROUTINES}

The COBRA/CFTL code is expected to be used at ORNL for specific application to CFTL with helium coolant. Hence, a number of subroutines related to coolants other than helium were removed from the COBRA*GCFR code. In addition, subroutines related to rod conduction and radiation heat transfer calculations were modified (Ref. 6). Table 1 1ists the subroutines that were removed from the COBRA*GCFR code and the subroutines that were added.

\subsection{USE OF MODIFIED $R\left(h^{+}\right)$AND $G\left(h^{+}\right)$FUNCTIONS}

As discussed in kefs. 3 through 5 , the CUBKA/LrIL code uses the following velocity and temperature profiles in the rough portion of the bundle:

$$
\begin{aligned}
& u^{+}=\operatorname{ARen}\left(Y / h_{R}\right)+R\left(h^{+}\right) \\
& t^{+}=\operatorname{AHen}\left(Y / h_{R}\right)+G\left(h^{+}\right)
\end{aligned}
$$

The correlations for the $R\left(h^{+}\right)$and $G\left(h^{+}\right)$functions and the slopes of the velocity and temperature profiles are of the form

\section{$\underline{R\left(h^{+}\right) \text {and } A R}$}

1. Fully Rough Flow: $\left[h_{w}^{+}>C R(3)\right]$

$$
\begin{aligned}
R\left(h^{+}\right) & =C R(1) \\
A R & =C R(2)
\end{aligned}
$$


2. Transition Flow:

$$
\begin{aligned}
R\left(h^{+}\right) & =C R(4)-C R(5) \ln \left(h^{+}\right) \\
A R & =C R(6)-C R(7) \ln \left(h^{+}\right)+C R(8)\left[\ln \left(h^{+}\right)\right]^{2}
\end{aligned}
$$

3. Hydraulically Smooth Flow:

$$
\begin{aligned}
{\left[R\left(h^{+}\right)+(A R-2.5)\left\{\text { en } \frac{Y_{L}}{h_{R}}-0.5-\frac{1}{2+\frac{Y_{L}}{r_{1}}}\right)\right]>\left[2.5 \ln \left(h_{B}^{+}\right)+5.5\right] } \\
R\left(h^{+}\right)=5.5+2.5 \ln \left(h_{B}^{+}\right) \\
A R=2.5
\end{aligned}
$$

\section{$\underline{G\left(h^{+}\right) \text {and } A H}$}

For $h_{w}^{+} \geq C G(6)$ :

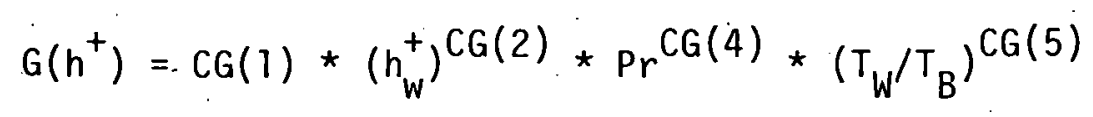

$$
\text { For } \begin{aligned}
h_{W}^{+} & <C G(6): \\
G\left(h^{+}\right) & =C G(7) \\
A H & =C G(3)
\end{aligned}
$$

The coefficients $C R(1)$ to $C R(8)$ and $C G(1)$ to $C G(7)$ in Eqs. 3 to 11 depend on the roughness geometry as given in Refs. 3 through 5 and are.input to the code.

\subsection{STANTON NUMBER IN INTERIOR CHANNELS}

The COBRA*GCFR code calculates the Stanton number in the interior channel (see Fig. 1) from Eqs. 1 and 2 by using the Dipprey-Saberskey heat 
transfer similarity law (Ref. 7). This has been replaced in the COBRA/CFTL code by the relation obtained by solving the equation.

$$
\frac{\sqrt{f_{1} / 2}}{S t_{1}}=A H\left[\ln \left(Y_{L} / h_{R}\right)-0.5-1 /\left(2+Y_{L} / r_{1}\right)\right]+G\left(h^{+}\right)
$$

\subsection{MODIFICATIONS FOR VARIABLE SLOPES AR AND AH}

In the COBRA*GCFR code (Ref. 2), it is assumed that

$$
A R=A H=2.5
$$

However, as discussed above, in the COBRA/CFTL code AR and AH are calculated from input quantities. Hence, a number of modifications were made to subroutines BASIC and IDDT to accommodate this change.

\subsection{SI (METRIC) UNITS}

A number of appropriate changes have been made so that all the input, the internal calculations, and the output are in SI units.

\subsection{LAMINAR NUSSELT NUMBERS}

In the COBRA*GCFR code, correlations for laminar heat transfer coefficients are part of the input. This has been modified and the following correlations from Ref. 8 have been made part of the coding in the COBRA/ CFTL code for laminar flow calculations.

Interior Channels

$$
\mathrm{Nu}=\frac{\alpha}{1+\alpha}\left[14.12+4.13 \text { en }\left(\frac{0.95}{\alpha-1}\right)\right]
$$

Wall and Corner Channels

$$
\mathrm{Nu}=4.07+\frac{1.24}{(\alpha)^{0.803}}
$$

where $\alpha=r_{1} / r_{2}$ for wall. and corner channels

$$
=r_{1} / r_{0} \text { for interior channels. }
$$




\subsection{SHROUD TEMPERATURE DURING LAMINAR FLOW}

The COBRA*GCFR code calculates shroud temperatures only when $R\left(h^{+}\right)$ and $G\left(h^{+}\right)$functions are used for friction factor and heat transfer coefficient calculations. In the COBRA/CFTL code, a method of the SAGAPO code (Ref. 8) has been incorporated to calculate the shroud temperatures during laminar flow. However, there seems to be an error in the programing of this calculation. Results of the test Cases 2 and 3 (presented in later in tiin report) indicate that the COBRA/CFTL code calculates the shroud temperature to be less than the inlet coolant temperature at a number of locations during laminar flow. This is possible only when there is a heat loss from the shroud. In these test cases, no such heat loss was considered.

\subsection{EFFECT OF SPACERS ON HEAT TRANSFER DURING LAMINAR AND TRANSITION FLOW}

The correlations for this effect were not available when development work on the COBRA*GCFR code was stopped in. February 1980. These correlations from Ref. 9 have been included in the COBRA/CFTL code.

$$
\begin{aligned}
& \text { Upstream and Under-Space Effect }\left(-1 \leq \frac{x}{D_{H}}<A D\right) \\
& \qquad N u^{*}=1+\frac{N{ }^{*}{ }_{\max }-1}{A D+1}\left(\frac{x}{D_{H}}+1\right)
\end{aligned}
$$

For Rough Surface: $N u_{\max }^{\star}=1+K(\operatorname{Re}) \varepsilon^{2}$

$$
\begin{aligned}
& \text { where } K(\operatorname{Re})= \begin{cases}.174 \cdot \operatorname{Re}^{.5} & \operatorname{Re}<2653 \\
3.3+72700 \operatorname{Re}^{-1.2} & \operatorname{Re}>2653\end{cases} \\
& A D=\frac{S H / 2}{D_{H}}
\end{aligned}
$$

For Smooth Surface:

$$
N u_{\max }^{*}=\operatorname{Min}\left\{\begin{array}{l}
1 .+.174 \operatorname{Re}^{: 5} \varepsilon^{2} \\
1 .+\left(6.38+4550 \mathrm{Re}^{-0.8}\right) \varepsilon^{2 \cdot 4}
\end{array}\right.
$$




$$
A D= \begin{cases}\frac{S H / 2}{D_{H}} & R e<3000 \\ S H / D_{H} & R e>3000\end{cases}
$$

Downs tream of Spacer $\left(\frac{x}{D_{H}}>A D\right)$

$$
N u^{*}=K *\left(\frac{x / D_{H}}{\operatorname{RePr}}\right)^{m}
$$

For Rough Surface:

$$
\begin{aligned}
& m= \begin{cases}1.1855 \times 10^{-3} \operatorname{Re} \varepsilon^{2} & \operatorname{Re}<3000 \\
\left(1+4.9 \times 10^{4} \operatorname{Re}^{-1.2}\right) \varepsilon^{2} & \operatorname{Re}>3000\end{cases} \\
& m>-4 \varepsilon^{2}, \quad m=-4 \varepsilon^{2} \\
& K=\left\{\begin{array}{lr}
4.42-1.05 \log _{10}(\operatorname{Re})-2.25 \varepsilon & \operatorname{Re} 3000 \\
-.1298\left[\log _{10}(\operatorname{Re})\right]^{2}+1.2466\left[\log _{10}(\operatorname{Re})\right]-1.8478-2.25 \varepsilon ; \operatorname{Re}>3000
\end{array}\right. \\
& K<.885-2.25 \varepsilon, \quad K=.885-2.25 \varepsilon \text {. }
\end{aligned}
$$

For Smooth Surface:

$$
\begin{aligned}
& m= \begin{cases}-1.855 \times 10^{-3} \operatorname{Re} \varepsilon^{2} & \operatorname{Re}<3000 \\
-30.34 \mathrm{Re}^{-0.253} \varepsilon^{2} & \operatorname{Re}>3000\end{cases} \\
& m<-4 \varepsilon^{2}, \quad m=-4 \varepsilon^{2}
\end{aligned}
$$




$$
\begin{aligned}
& K= \begin{cases}4.42-1.05 \log _{10}(\operatorname{Re})-2.25 \varepsilon & \operatorname{Re}<3000 \\
.426+.113 \log _{10}(\operatorname{Re})-2.25 \varepsilon & \operatorname{Re}>3000\end{cases} \\
& K<.895-2.25 \varepsilon, \quad K=.885-2.25 \varepsilon
\end{aligned}
$$

\subsection{INPUT/OUTPUT CHANGES}

A number of changes in input and output have been made for convenience. Appendix $A$ describes the input instructions and Appendices $B$ and $C$ show sample input/output from the COBRA/CFTL code.

\subsection{SEARCH FOR STEP CHANGE IN HEAT FLUXX}

In the COBRA*GCFR code, a search for a step change in heat flux between nodes consists essentially of a change from zero to a non-zero value or vice-versa. This was modified such that the test in COBRA/CFTL is for a factor of three increase or decrease in heat flux at adjacent nodes. If the test is positive, the distance between nodes is subdivided into 20 parts for accurate calculation of the energy added. Also, this modification has altered the definition of "location of the start of heating:" In the COBRA*GCFR code, the heating is assumed to start whenever heat flux is non-zero. However, COBRA/CFTL defines the start of heating when heat flux has a factor of three increase, as explained above. This difference in the definition of "location of start of heating" leads to significant differences in laminar heat transfer coefficients in the developing region.

\subsection{CORRECTION FOR SMOOTH GEOMETRY}

Since the more important calculations in the codes are in the rough portion of the bundle, the geometry of the rough part of the bundle (i.e., flow area, wetted perimeter, etc.) is an input to the codes. The flow area and wetted perimeter for the smooth part of the bundle differ from those of the rough part of the bundle. The flow area can be correctly accounted for by using the axial flow area variation option in the codes. Programming has been provided in the COBRA/CFTL code to reduce the hydraulic diameter by $1.7 \%$ whenever the flow area variation factor is less than 0.99 . The $1.7 \%$ correction to the hydraulic diameter represents the average for the CFTL test bundles. 


\subsection{PROPERTY TABLE RANGE}

The subroutine SETUP was modified to permit setting up minimum temperatures in the property table in the event that individual subchannel temperatures and enthalpies are read in.

\subsection{TEST FOR LAMINAR FRICTION FACTOR}

Changes were made to subroutines PROP, SETUP, and SETUP2 to test the turbulent friction factor value (FSP(I)) against the laminar friction factor value (FLAM) whenever the index LAMNF >0. If FLAM < FSP(I), the flow is assumed to be turbulent.

\subsection{IMPOSITION OF LAMINAR FLOW CALCULATIONS}

When the laminar option (Card 2A) is selected, the COBRA/CFTL code checks whether the flow is laminar or turbulent in a given channel by calculating and comparing the laminar and turbulent friction factors. In some instances the flow rate is low enough that this test is unnecessary. The code has been modified to permit the user to select an option so that only laminar calculations are performed ( LAMNF $=2$ ).

\subsection{CORRECTION FOR AXIAL FLOW WHEN ROD CONDUCTION CALCULATIONS ARE MADE}

The heat fluxes computed in the subroutine RODCON (see Ref. 6). are at the midpoint between the nodes. However, the clad temperature in the COBRA/CFTL code are at the nodes. The code has been modified to adjust the flux values to the node locations for clad temperature calculations. This adjustment is required only for the case in which rod conduction calculations are made.

\subsection{CORRECTION TO BUNDLE-AVERAGED RESULTS}

In the COBRA/CFTL and the COBRA*GCFR codes, heat transfer to and from the bundle is modeled by creating a dummy flow channel outside the bundle. In the COBRA*GCFR code, the parameters of the dummy channel are included in the bundle-averaged results. However, in the COBRA/CFTL code an option has been added such that the dummy channel can be excluded from the bundle-average calculation by specifying that it is of a type greater than NFT (Card 4B).

\subsection{SYMMETRY OPTION}

The symmetry option in the COBRA*GCFR code consisted of making the adjacent channel number ( $L C(I, J))$ in group 4 negative at the symmetry lines. This adjusted the cross flow and conduction through the coolant to $50 \%$ of the actual values. This option has been removed in the COBRA/CFTL code. 


\subsection{REMOVAL OF UNNECESSARY OPTIONS}

The following options, which were considered unnecessary for the CFTL calculations or for the ORNL computer, were removed from the COBRA* GCFR code.

1. COBRA-IV fuel rod model

2. Roll option

3. Restart option

4. Coolant properties other than helium 


\section{COMPARISON OF COBRA*GCFR AND COBRA/CFTL RESULTS}

To investigate the effect of various changes discussed in Section 2, calculation results of the COBRA*GCFR and the COBRA/CFTL codes were compared for three tests which covered the turbulent flow, transition flow, hydraulically smooth, and laminar flow regimes. The test section considered was a sixth section (Fig. 1) of the AG-1 bundle designed for testing in the CFTL (Ref. 1). The test conditions are summarized in Table 2.

\section{1 TEST 1}

The flow conditions for Test 1 (Table 2) are fully turbulent rough flow in all channels. The results of the two codes are likely to be affected by different forms of $R\left(h^{+}\right)$and $G\left(h^{+}\right)$functions.

Figures 2 through 7 show the comparison of results for this test. The pressure drop and coolant temperature calculations in all channels agree very closely. The maximum deviation in these quantities is less than $1 \%$. Similarly, there is a close agreement in rod surface temperatures of interior and corner channels.

However, rod and shroud temperatures in the wall channel (Figs. 4 and 5) differ by as much as $20^{\circ} \mathrm{C}$ (out of a film drop of $200^{\circ} \mathrm{C}$ ). The procedure of inverse transformation used for calculating the Stanton numbers in the wall channel (Ref. 4) indicates that this difference is likely to be due to different forms of $R\left(h^{+}\right)$and $G\left(h^{+}\right)$functions used in the two codes. The difference in the calculated shroud temperatures is also due to different forms of the $R\left(h^{+}\right)$and $G\left(h^{+}\right)$functions.

\subsection{TEST 2}

The flow conditions for Test 2 are such that the flow is hydraulically smooth in the interior channel and laminar in the corner and wall channels. Figures 8 through 13 show the comparison of the results obtained by the two codes.

The pressure drop results (Fig. 8) are within $5 \%$. The slight difference is due to the difference in the calculated friction factors in the interior channels. Similarly, there is close agreement in coolant temperature (Figs. 9 through 13) in all channels. This indicates that the flow distribution calculated by the two codes was quite close.

However, there is substantial disagreement in the rod surface temperatures in all channels. This is due to the different correlations for the 
$R\left(h^{+}\right)$and $G\left(h^{+}\right)$functions used by the two codes in this flow regime and different correlations for space enhancement of heat transfer. The correlations used in COBRA/CFTL are based on published results which were not available when development of COBRA*GCFR code was stopped.

Another difference in the two codes which affects the heat transfer coefficients is the definition of the start of heating point. For the axial heat flux under consideration, COBRA*GCFR calculates the start of heating at $x=0$, whereas COBRA/CFTL calculates the start of heating at $x=939 \mathrm{~mm}$. This affects the laminar heat transfer coefficient, since this coefficient is a function of distance from the start of heating point (Ref. 2).

There is a large disagreement in the shroud temperatures calculated by the two codes (Fig. 11). However, shroud temperatures calculated by COBRA/CFTL are obviously in error since some of the shroud temperatures are lower than the inlet temperatures. This must have been due to some programming error in implementing the methods of the SAGAPO code.

\subsection{TEST 3}

The flow conditions for Test 3 are such that the flow is in the transition regime in all channels. Figures 14 through 19 show the comparison of results.

Similar to Test 2, the results from the two codes for pressure drop and coolant temperatures agree well. Also, there is a close agreement in the rod surface temperatures for the interior channels. However, rod surface temperature for wall and corner channels differ because of different $R\left(h^{+}\right)$and $G\left(h^{+}\right)$functions and different spacer enhancement correlations.

The remarks about shroud temperature calculations for Test 2 also apply to the results of this test.

\subsection{SUMMARY OF CODE COMPARISON}

The results of the code comparison have shown that the pressure drop and coolant temperature calculations agree closely for all flow conditions. The close agreement in the interior channel rod temperature for fully turbulent rough flow is not surprising. Because although the form of $R\left(h^{+}\right)$ and $G\left(h^{+}\right)$functions used in the lwo codes is different, the correlations are such that the values of the $R\left(h^{+}\right)$and $G\left(h^{+}\right)$. functions for fully turbulent flow are equal. The codes predict different results for rod surface temperatures and duct temperatures, especially during laminar and transition flow due to different correlations for the $R\left(h^{+}\right)$and $G\left(h^{+}\right)$functions, different definitions for the start of heating point, a programming error in the COBRA/CFTL code in laminar shroud temperature calculations, and different spacer enhancement correlations. Table 3 summarizes these comparison results. 


\section{COST COMPARISONS}

Table 4 compares costs and standard units of processing (SUP) time on the COBRA/CFTL and COBRA*GCFR codes. For Test 1 , costs and SUP times are very close. For Tests 2 and 3 , costs on the COBRA*GCFR code are about $70 \%$ of those on the COBRA/CFTL code. The reason for this may be that the COBRA/CFTL code used $R\left(h^{+}\right)$and $G\left(h^{+}\right)$functions for Tests 2 and 3 , where as COBRA*GCFR uses input correlations for Tests 2 and 3 . Use of $R\left(h^{+}\right)$and $G\left(h^{+}\right)$functions result in added iteration for convergence and, hence, added costs.

Interestingly, reducing the size of the code by removing a number of subroutines (Table 1) did not reduce the time or computation cost of the code on the Univac computer at GA. However, the shortened code does provide substantial benefit when run on the IBM computer at ORNL. 


\section{SUMMARY AND CONCLUSIONS}

The COBRA/CFTL code has been developed for thermal-hydraulic analys is of simulated GCFR core assemblies to be tested in the CFTL. The code was obtained by modification of the COBRA*GCFR code. These modifications consisted of:

1. Removing of unnecessary routines.

2. Changing velocity and temperature profile correlations.

3. Changing the method of calculating Stanton number in the interior channels.

4. Converting to SI units.

5. Building-in laminar Nusselt number correlations.

6. Including shroud temperature calculation during laminar flow.

7. Incorporating effect of spacer on heat transfer coefficient during laminar flow.

8. Incorporating changes in input/output.

9. Changing the method for step change in heat flux.

10. Correcting for smooth geometry.

Some of the above changes were made for convenience; others were made to include more up-to-date information in the code. Comparing the calculations of the two codes shows that

1. Pressure drop calculations for all flow conditions agree well.

2. Coolant temperatures and flow distributions also agree well for all flow conditions.

3. The rod and shroud temperatures in the wall channel during fully turbulent rough and transition flow differ because of different forms of $R\left(h^{+}\right)$and $G\left(h^{+}\right)$functions.

4. The rod temperatures during laminar flow differ because of code differences in the spacer enhancement correlation and in the definition of the start of heating point. 
5. Shroud temperatures during the laminar flow differ because the COBRA/CFTL code uses the method from the SAGAPO code for this calculation, whereas the COBRA*GCFR code assumes the shroud temperature to be equal to the coolant temperature during laminar flow. The difference in this result seems to be due to a programming error in the COBRA/CFTL code.

Some of the modifications incorporated in the COBRA/CFTL code are clearly improvements over the COBRA*GCFR code (e.g., laminar space enhancement, correction for smooth region, shroud temperature calculation during laminar flow, method for step change in heat flux). 0ther changes connected with the new form of $R\left(h^{+}\right)$and $G\left(h^{+}\right)$functions and variable slopes of the velocity and temperature profiles are based on the development work done at ORNL over the last three years. The procedure suggested has been supported to some extent by recent experiments at KfK. Unfortunately, because of the termination of the GCFR program, comparison of both code calculations with same bundle experiment has not been done. 


\section{REFERENCES}

1. Gat, U. and A. C. Grinde11, "The Core Flow Test Loop (CFTL) GasCooled Fast Reactor Program," May 30 - June 1, 1979, Rancho Bernardo, California, Helium Breeder Associates (1979).

2. Baxi, C. B., F. 0. Bennett, C. J. Burhop, and G. Schlueter, "COBRA*GCFR: A. Computer Code for Steady-State and Transient Thermal-Hydraulic Analysis of GCFR Core Assemblies," General Atomic Report GA-A16002, September 1980.

3. Hodge, S. A., J. P. Sanders, and D. E. Klein, "Determination of Friction Factors and Heat Transfer Coefficients for Flow Past Artifically Roughened Surfaces," ORNL-5599 (1979).

4. Hodge, S. A., J. P. Sanders, and D. E. Klein, "Slope and Intercept of the Dimensionless Velocity Profile for Artificially Roughened Surfaces," Int. J. Heat Mass Transfer, 23, 135-140 (1980).

5. Hodge, S. A., "Determination of Coefficients for the Universal Laws of Friction and Heat Transfer for CFTL Calculations, "ORNL Report ORNL/TM-7418, August 1980.

6. Conklin, J. C., "Development and Adaption of Conduction and Radiation Heat Transfer Computer Codes for the CFTL," ORNL/TM-7854, July 1981.

7. Dipprey, D. F. and D. H. Sabersky, "Heat and Momentum Transfer in Smooth and Rough Tubes at Various Prandt1 Numbers," Int. J. Heat Mass Transfer, $\underline{6}, 329-353$ (1963).

8. Martel1i, A., "SAGAPO-2, An Improved Version of the SAGAPO Code: Main Features and New Capabilities," OECD-NEA Coordinating Group on Gas-Cooled Fast Reactor Development, Heat Transfer Specialist Meeting; Wurenlingen, Switzeriand, May 14-18, 1979.

9. Hassan, M. A., "Wärmeübergang in Abstanelshalterbereich Gasgekuhlter Stabbundel," Ph.D. Thesis, University of Karlsruhe, Federal Republic of Germany, February 1980. 
TABLE 1

SUBROUTINES REMOVED FROM AND ADDED TO. THE COBRA*GCFR CODE TO OBTAIN THE COBRA/CFTL CODE

\begin{tabular}{l|l}
\hline \multicolumn{1}{c|}{ Routines Removed } & \multicolumn{1}{|c}{ Routines Added } \\
\hline APF; BVOID; CHAIN; CHECK; & BLOCKDATA; CONROD; ELAPI; \\
CHF; CHFCOR; CHFI; CHF2; & ERROR; RODCON; SAXPY; \\
CIRCON; CLEAR; CO2; & SDOT; SPBFA; SPBSL; \\
DFAULT; DRIFT (dUMmY); & ZEROIT; ZERSET. \\
DUMPIT; ELAP; ERRORS; & \\
FILES; GAUSS; GROUP9; & \\
HCOOL; HTCOR; IAD; & \\
ISWAP; LESCON; LOADER; & \\
LOADL; LOCATE; LOCATR; & \\
LOOP; MOVEAB; NAMTAB; & \\
NEWTAB; PBOUND; READ; & \\
RESLTM; RESTRT; REWIND; & \\
ROLLIT; SCAN; SCQUAL; & \\
SIGN2, 3,4,5,7,8; SREAD; & \\
SRITE; STEAM; SIREAD; & \\
SIRITE; TEMP; TERM; \\
TODS; TRIDAG; VOID; \\
XSCHEM.
\end{tabular}


TABLE 2

TEST CONDITIONS FOR CODE COMPARISONS

\begin{tabular}{|c|c|c|c|c|c|c|c|}
\hline \multirow{2}{*}{$\begin{array}{c}\text { Test } \\
\text { No. }\end{array}$} & \multirow{2}{*}{$\begin{array}{l}\text { Bundle } \\
\text { Average } \\
\text { Re }\end{array}$} & \multicolumn{2}{|c|}{ Interior Channels } & \multicolumn{2}{|c|}{ Wall Channels } & \multicolumn{2}{|c|}{ Corner. Channel } \\
\hline & & $\operatorname{Re}$ & Flow & $\operatorname{Re}$ & Flow & $\operatorname{Re}$ & Flow \\
\hline 1 & 83,100 & 100,400 & $\begin{array}{c}\text { Fully } \\
\text { Turbulent } \\
\text { Rough } \\
\end{array}$ & 63,600 & $\begin{array}{c}\text { Fully } \\
\text { Turbulent } \\
\text { Rough }\end{array}$ & 44,800 & $\begin{array}{c}\text { Fully } \\
\text { Turbulent } \\
\text { Rough }\end{array}$ \\
\hline 2 & 3,310 & 4,120 . & $\begin{array}{l}\text { Hydraulically } \\
\text { smooth }\end{array}$ & 2,484 & $\begin{array}{l}\text { Hydraulically } \\
\text { smooth/laminar }\end{array}$ & ],720. & $\begin{array}{l}\text { Hydraul ically } \\
\text { smooth/laminar }\end{array}$ \\
\hline 3 & 6,000 & 7,420 . & Transition & 4,420 & $\begin{array}{l}\text { Transition } \\
\text { hydraulically } \\
\text { smooth }\end{array}$ & 3,100 . & $\begin{array}{l}\text { Transition } \\
\text { hydraulically } \\
\text { smooth }\end{array}$ \\
\hline
\end{tabular}


TABLE 3

SUMMARY OF CODE COMPARISON

\begin{tabular}{|c|c|c|c|c|c|c|}
\hline$\cdot$ & \multirow[b]{2}{*}{$\begin{array}{l}\text { Difference } \\
\text { in Pressure } \\
\text { Drop }(\%)\end{array}$} & \multicolumn{5}{|c|}{$\%$ Difference in Calculated Temperatures $(a)$} \\
\hline & & Coolant & $\begin{array}{l}\text { Interior } \\
\text { Channel } \\
\quad \text { Rod }\end{array}$ & $\begin{array}{l}\text { Wall } \\
\text { Channel } \\
\text { Rod }\end{array}$ & $\begin{array}{l}\text { Corner } \\
\text { Channel } \\
\text { Rod }\end{array}$ & $\begin{array}{l}\text { Wall. } \\
\text { Channel } \\
\text { Shroud }\end{array}$ \\
\hline $\begin{array}{l}\text { Test } 1 \\
\text { Fully turbulent } \\
\text { rough flow }\end{array}$ & 0.45 & 0.41 & 3.0 & 8.5 & 2.4 & 23.0 \\
\hline $\begin{array}{l}\text { Test } 2 \\
\text { Hydraulically } \\
\text { smooth/laminar } \\
\text { flow }\end{array}$ & 4.8 & 5.5 & 10.6 & 25.4 & 22.3 & $-(b)$ \\
\hline $\begin{array}{l}\text { Test } 3 \\
\text { Transition/ } \\
\text { hydraulically } \\
\text { smooth flow }\end{array}$ & 1. & 2.5 & 14.3 & 17.7 & 24.5 & $-(b)$ \\
\hline
\end{tabular}

\footnotetext{
(a) $\%$ difference $=-\frac{T_{1}-T_{2}}{T_{1}-T_{I}} \times 100$.
}

where,

(b)

For these cases, COBRA/CFTL code calculates the shroud temperatures less than the inlet temperatures:

$\mathrm{T}_{1}=$ temperature calculated by COBRA*GCFF code.

$\mathrm{T}_{2}=$ temperature calculated by COBRA/CFTL code.

$\mathrm{T}_{\mathrm{I}}=$ inlet temperature. 
TABLE 4

COMPARISON OF COMPUTER TIMES AND COSTS

\begin{tabular}{c|c|c|c|c}
\hline \multirow{2}{*}{ Test No. } & \multicolumn{2}{|c|}{ COBRA/CFTL at GA } & \multicolumn{2}{c}{ COBRA*GCFR at GA } \\
\cline { 2 - 5 } & $\begin{array}{c}\text { SUP } \\
\text { Time (Sec) }\end{array}$ & Cost (\$) & $\begin{array}{c}\text { SUP } \\
\text { Time (Sec) }\end{array}$ & Cost (\$) \\
\hline 1 & 290 & 34.39 & 269 & 32.13 \\
\hline 2 & 152 & 19.05 & 103 & 13.43 \\
\hline 3 & 155 & 19.36 & 102 & 13.43 \\
\hline
\end{tabular}




\section{Types of Channels}

(1) Interior

(2) Wall

(3) Corner

(4) Interior

(5) Interior

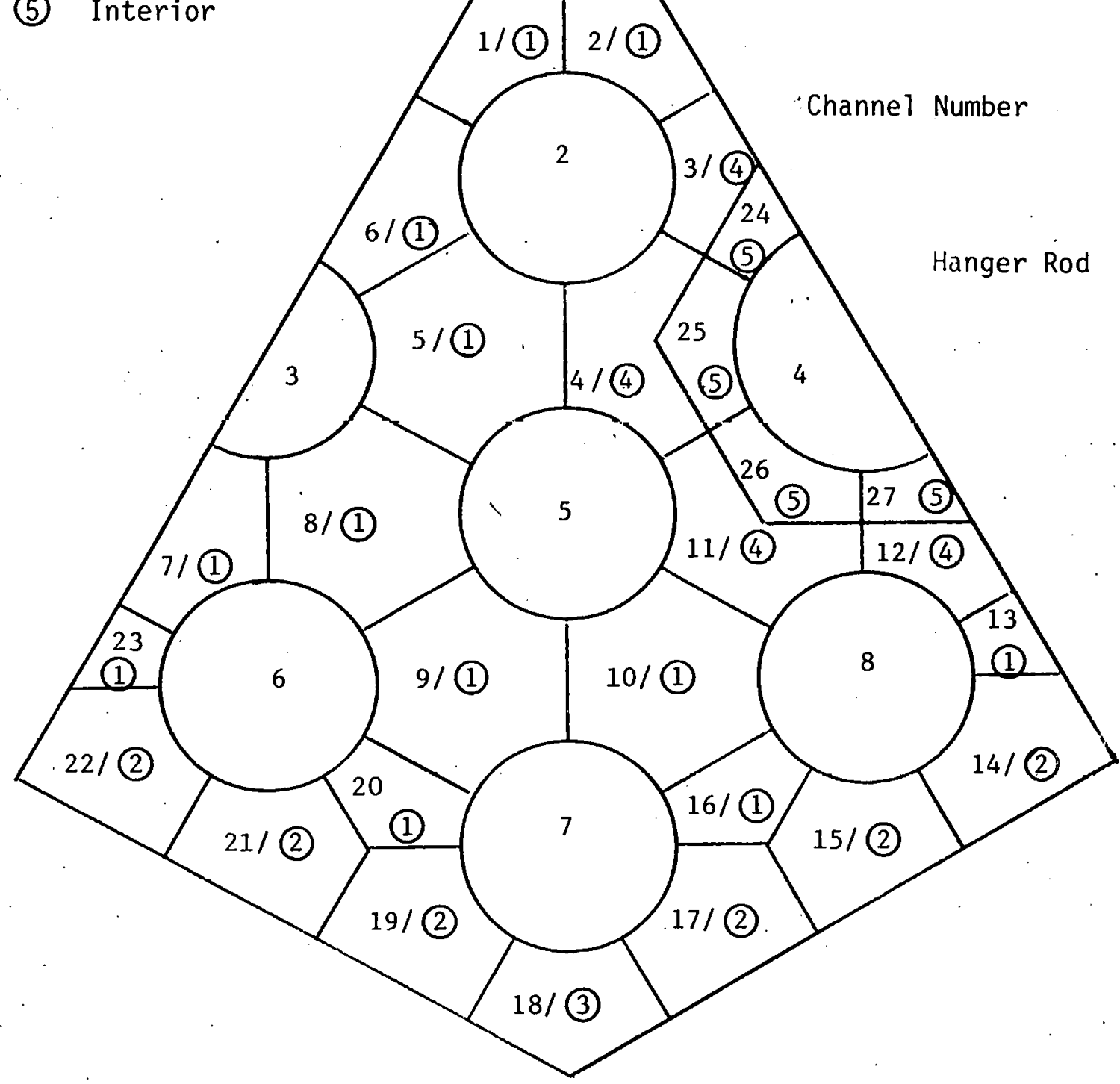

Figure 1. Subchannel and Rod Identification Numbers for the Test Bundle. 


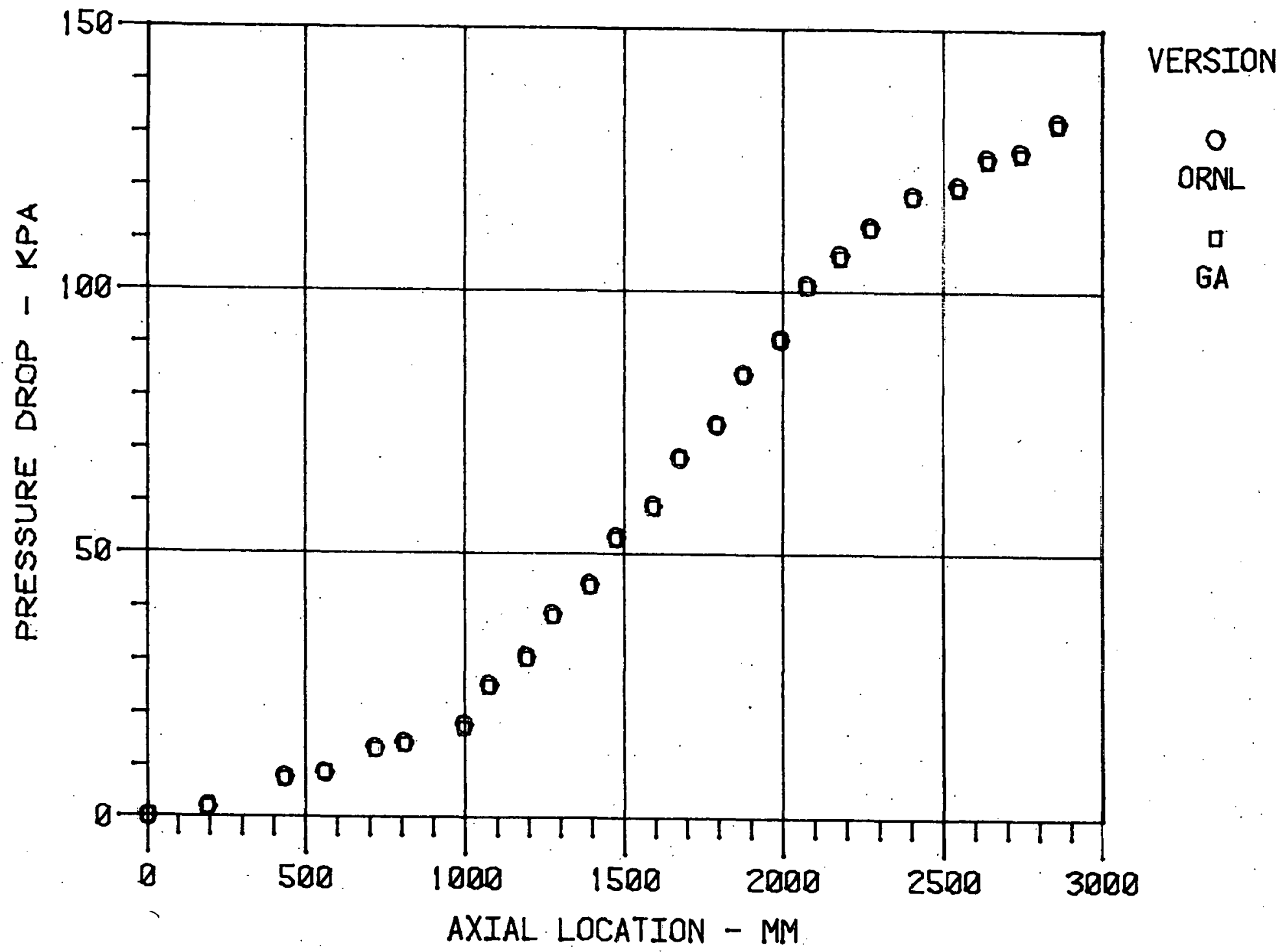

Fi.gure 2. Comparison of COBRA*GCFR and COBRA/CFTL Results - Test 1: Pressure Drop. 


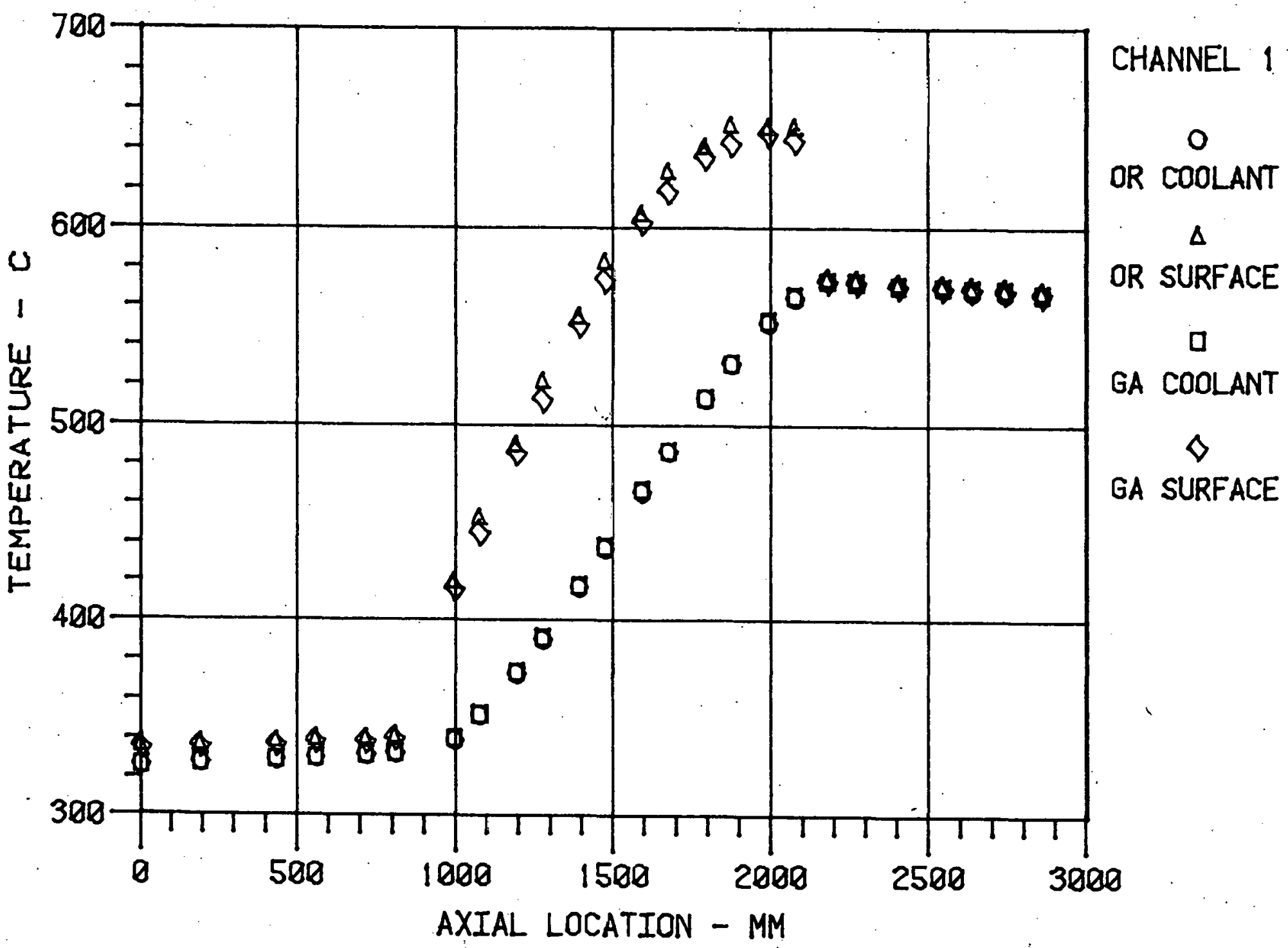

Figure 3. Comparison of COBRA*GCFR and COBRA/CFTL Results - Test 1: Coolant and Rod Surface Temperatures in Interior Channel. 


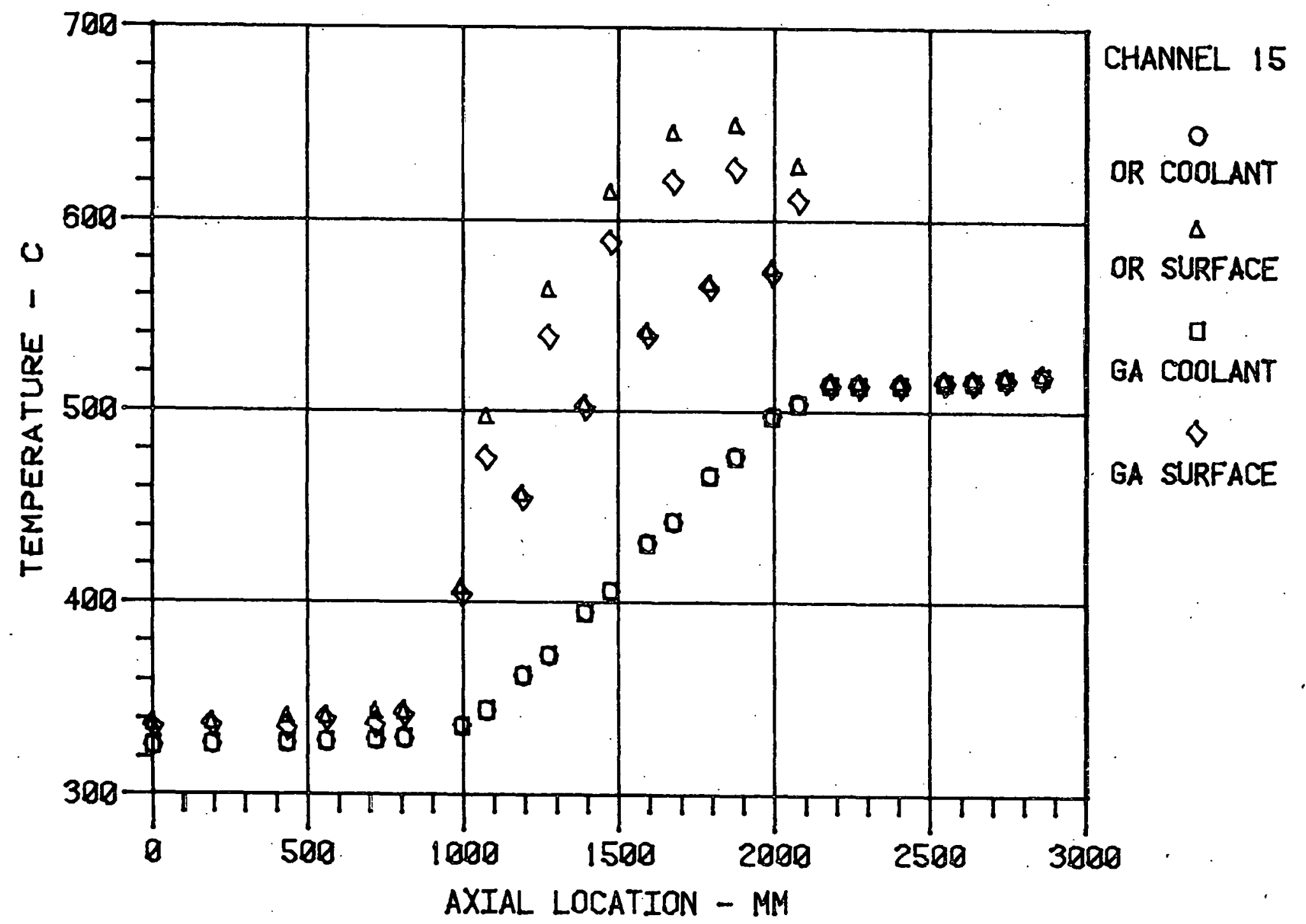

Figure 4. Comperison of COBRA*GCFR and COBRA/CFTL Results - Test 1: Coolent and Rod Surface Temperatures in Wall Channel. 


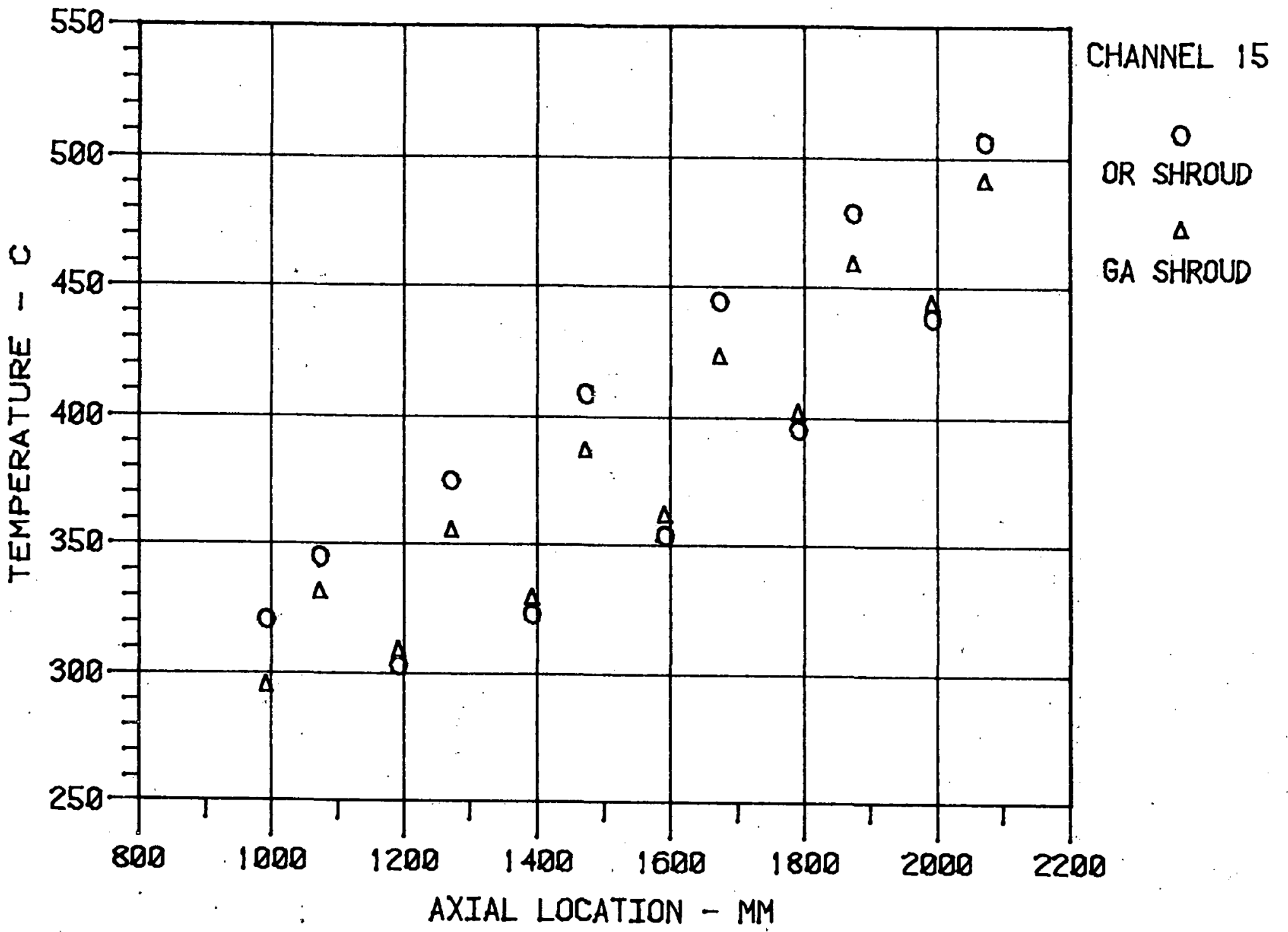

Figure 5. Comparison of COBRA*GCFR and COBRA/CFTL Results - Test 1: Shroud Temperatures in Wall Channel. 


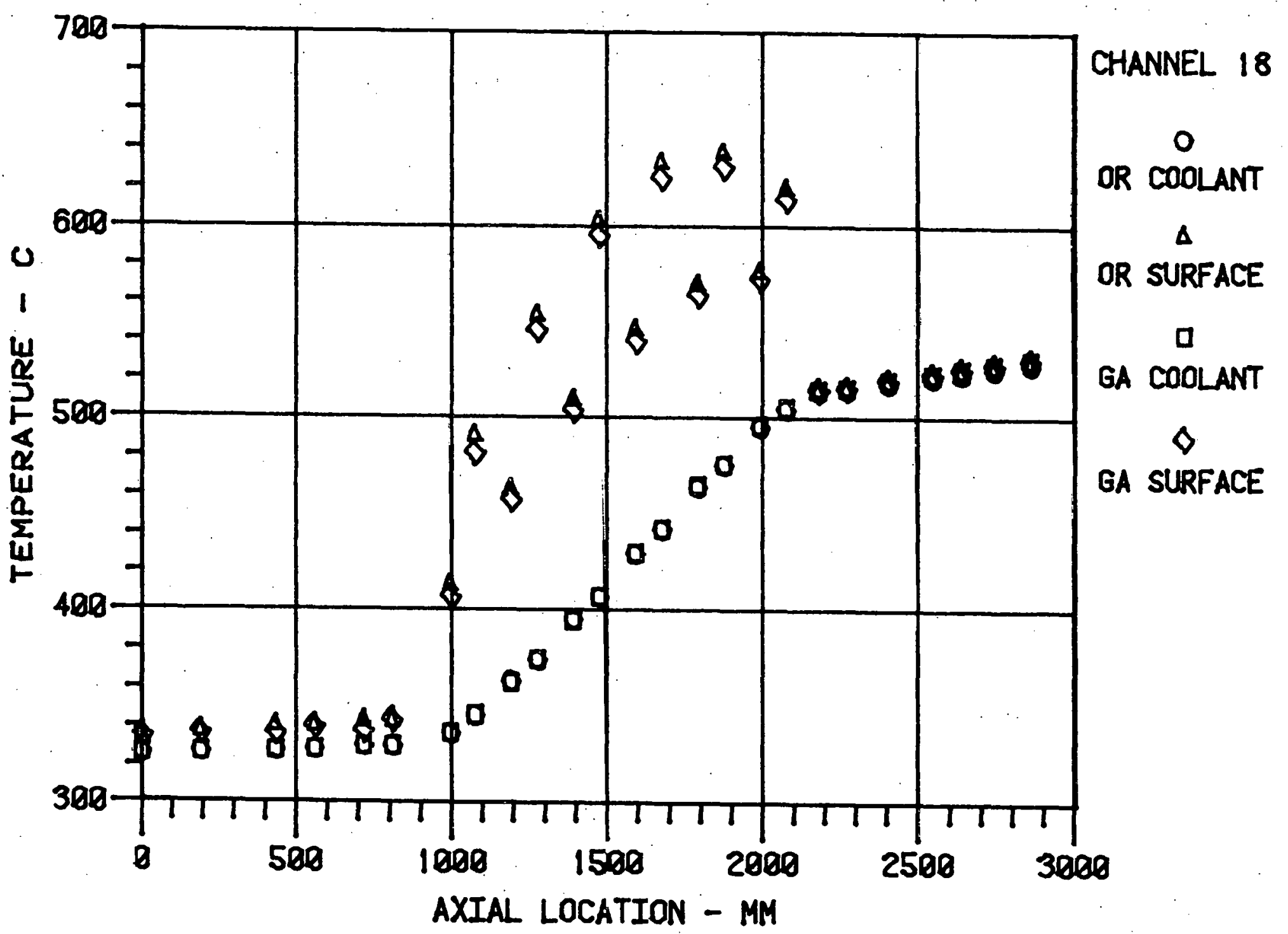

Figure 6. Comparison of COBRA*GCFR and COBRA/CFTL Results - Test 1: Coolant and Rod Surface Temperatures in Corner Channel. 


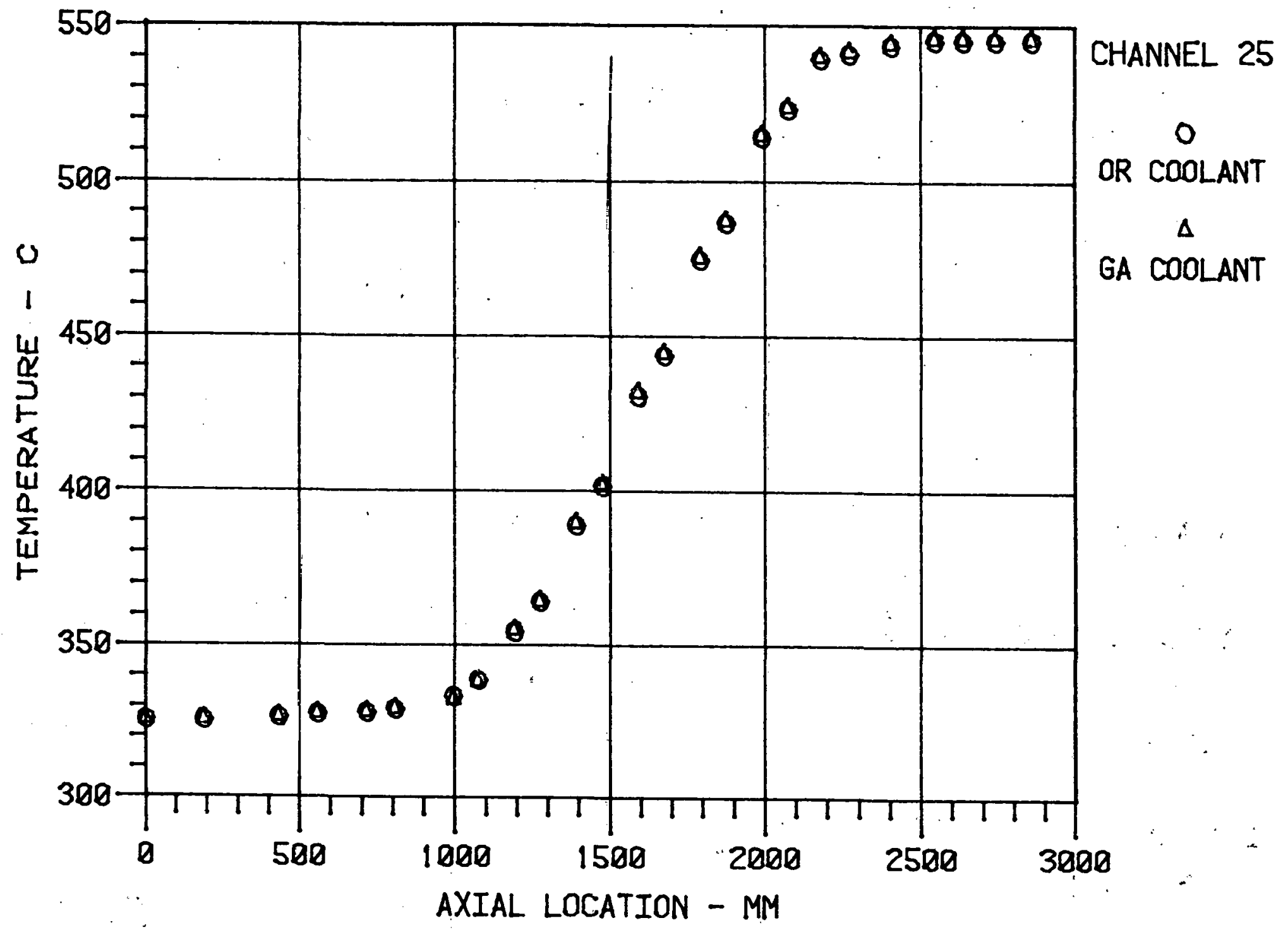

Figure 7. Comparison of COBRA*GCFR and COBRA/CFTL Results - Test 1: Coolant Temperatures in Channel Next to Hanger Rod. 


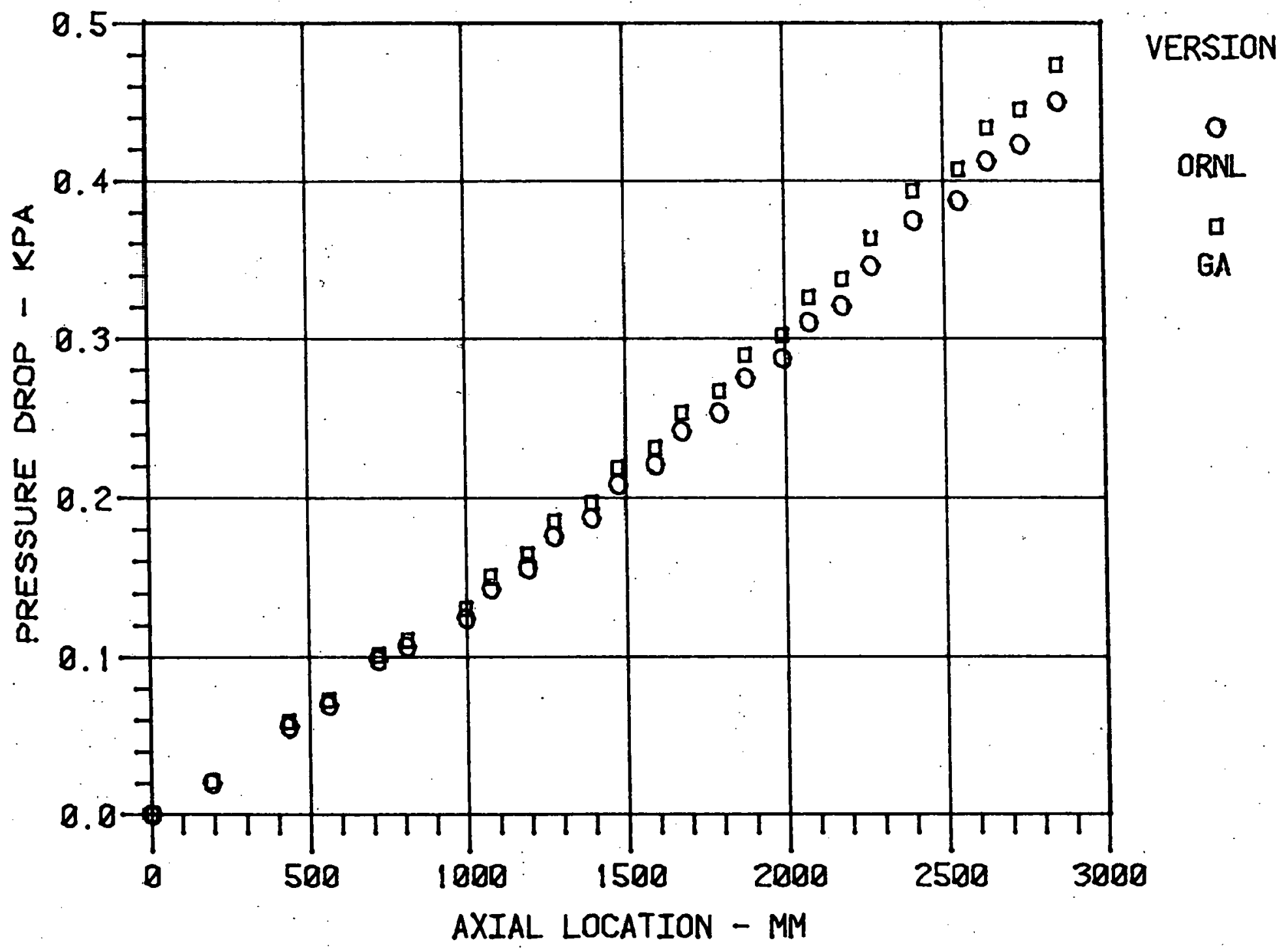

Figure 8. Comparison of COBRA*GCFR and COBRA/CFTL Results - Test 2: Pressure Drop. 


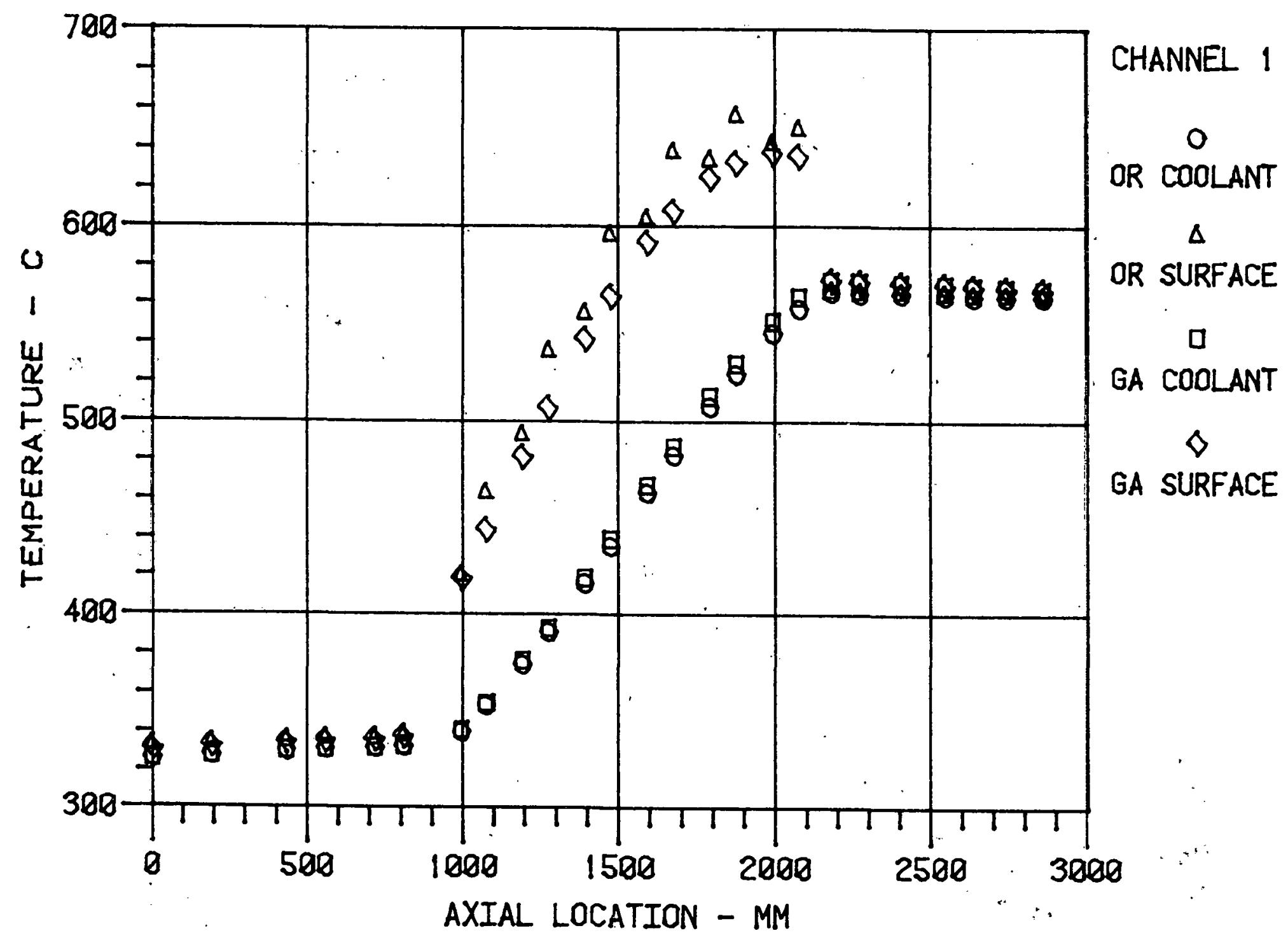

Figure 9. Comparison of COBRA*GCFR and COBRA/CFTL Results - Test 2: Coolant and Rod Surface Temperatures in Interior Channel. 


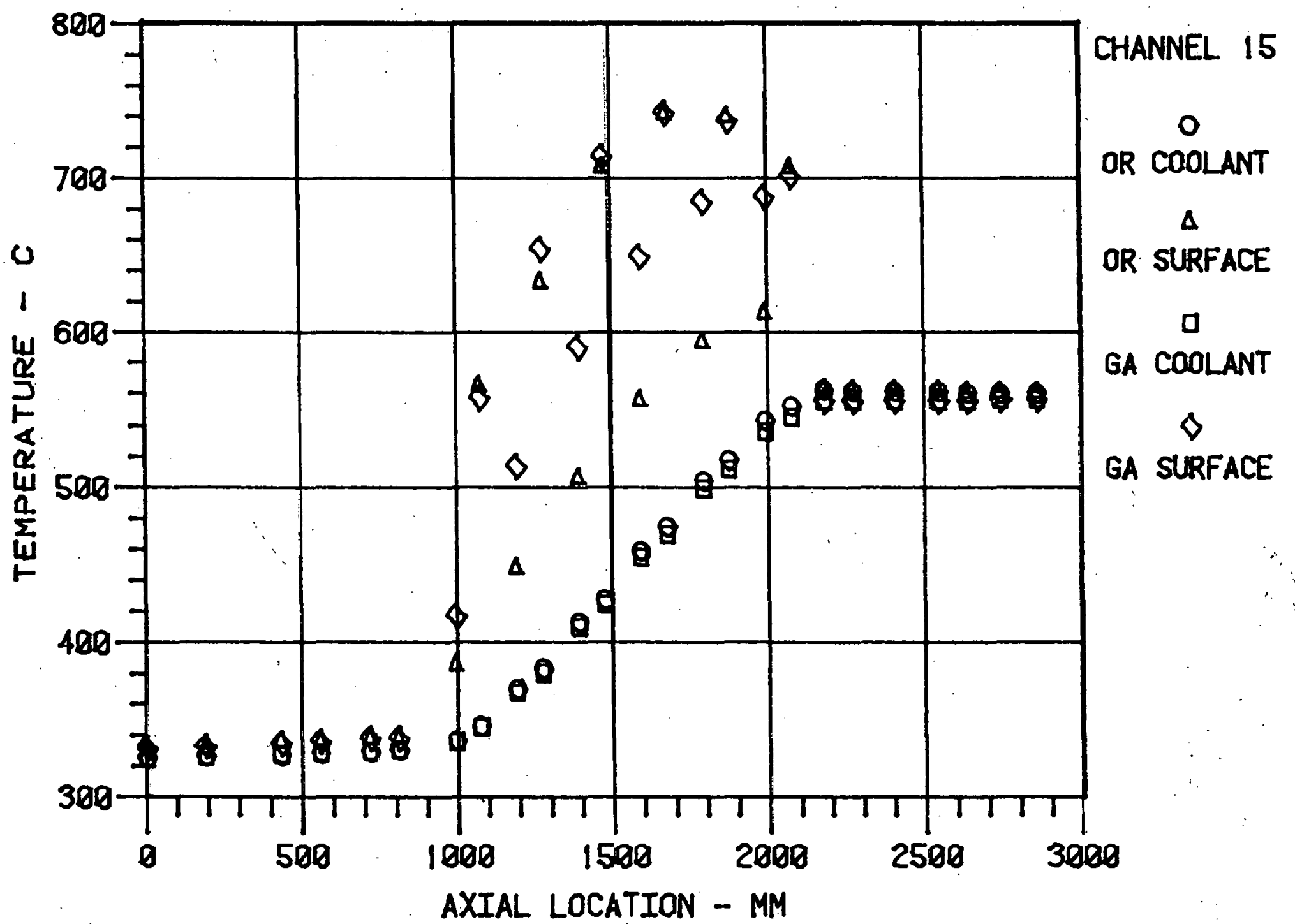

Fiçure 10. Comparison of COBRA*GC=R and COBRA/CFTL Results - Test 2: Coolant and Rod Surface Temperatures in Wall Channel. 


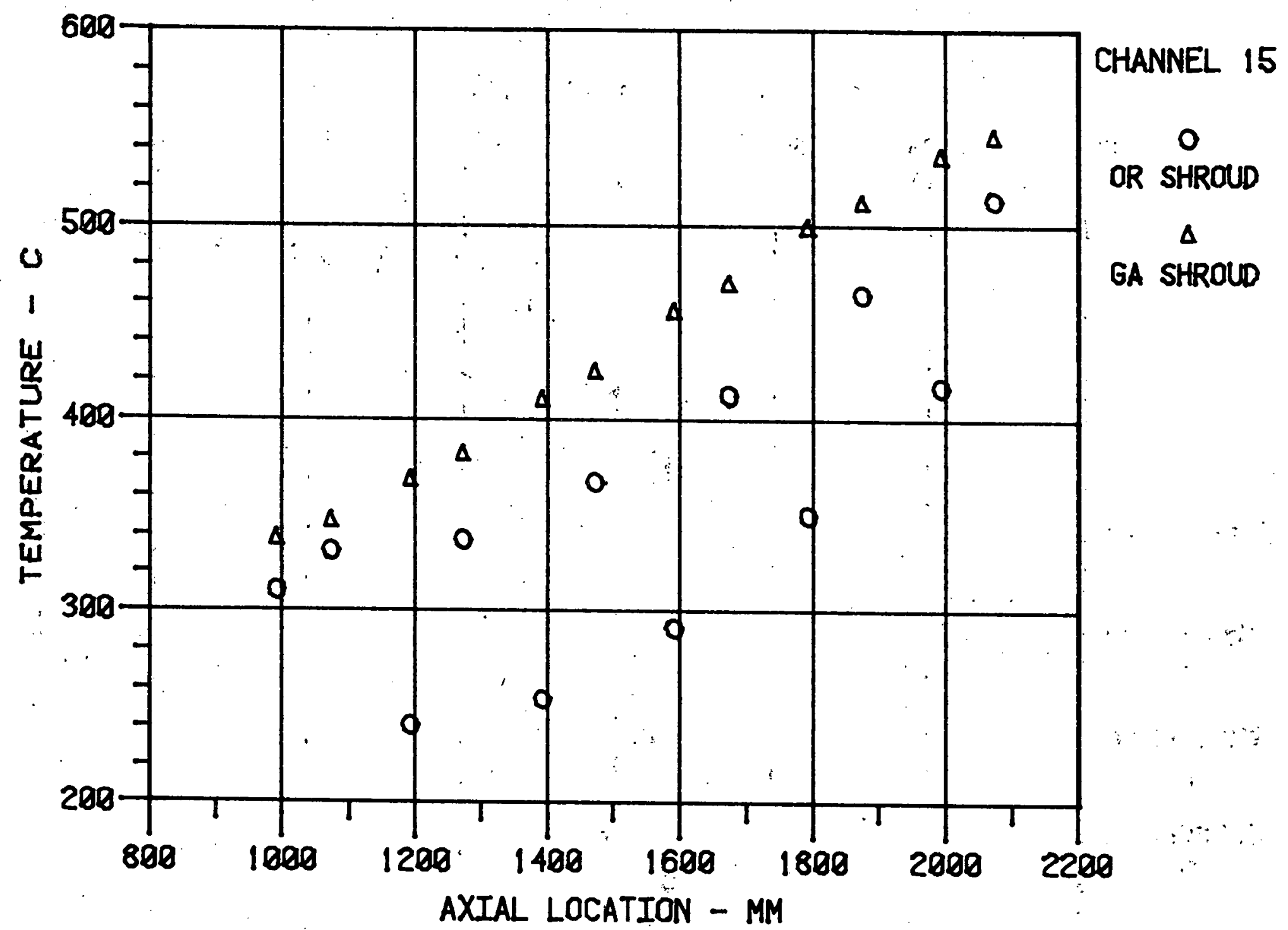

Figure 11. Comparison of COBRA*GCFR and COBRA/CFTL Results - Test 2: Shroud Temperatures ir Wal1. Channel. 


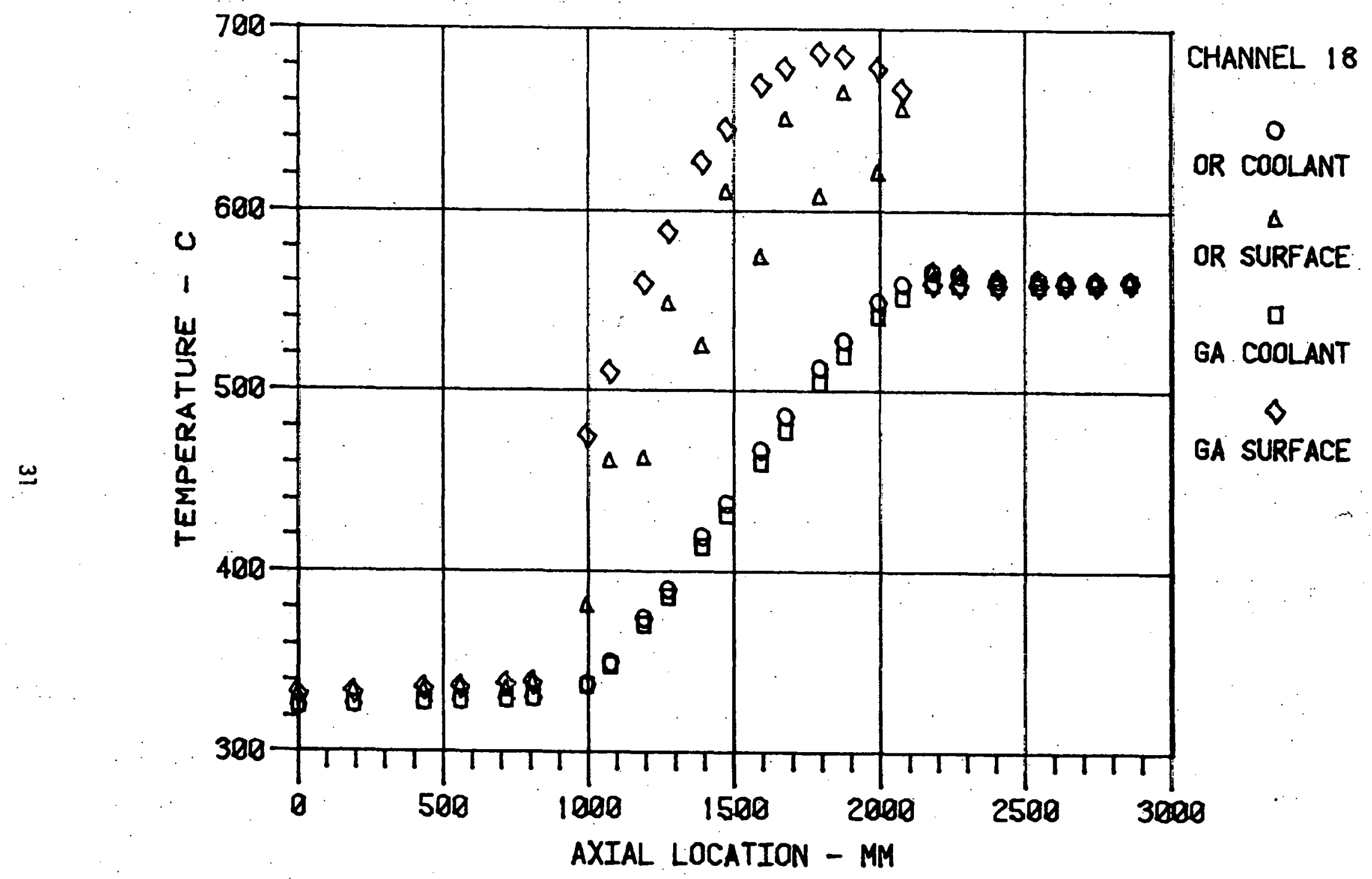

Figure 12. Comparison of COBRA*GCFR and COBRA/CFTL Results - Test 2: Coolant and Rod Surface Temperatures in Corner Channel. 


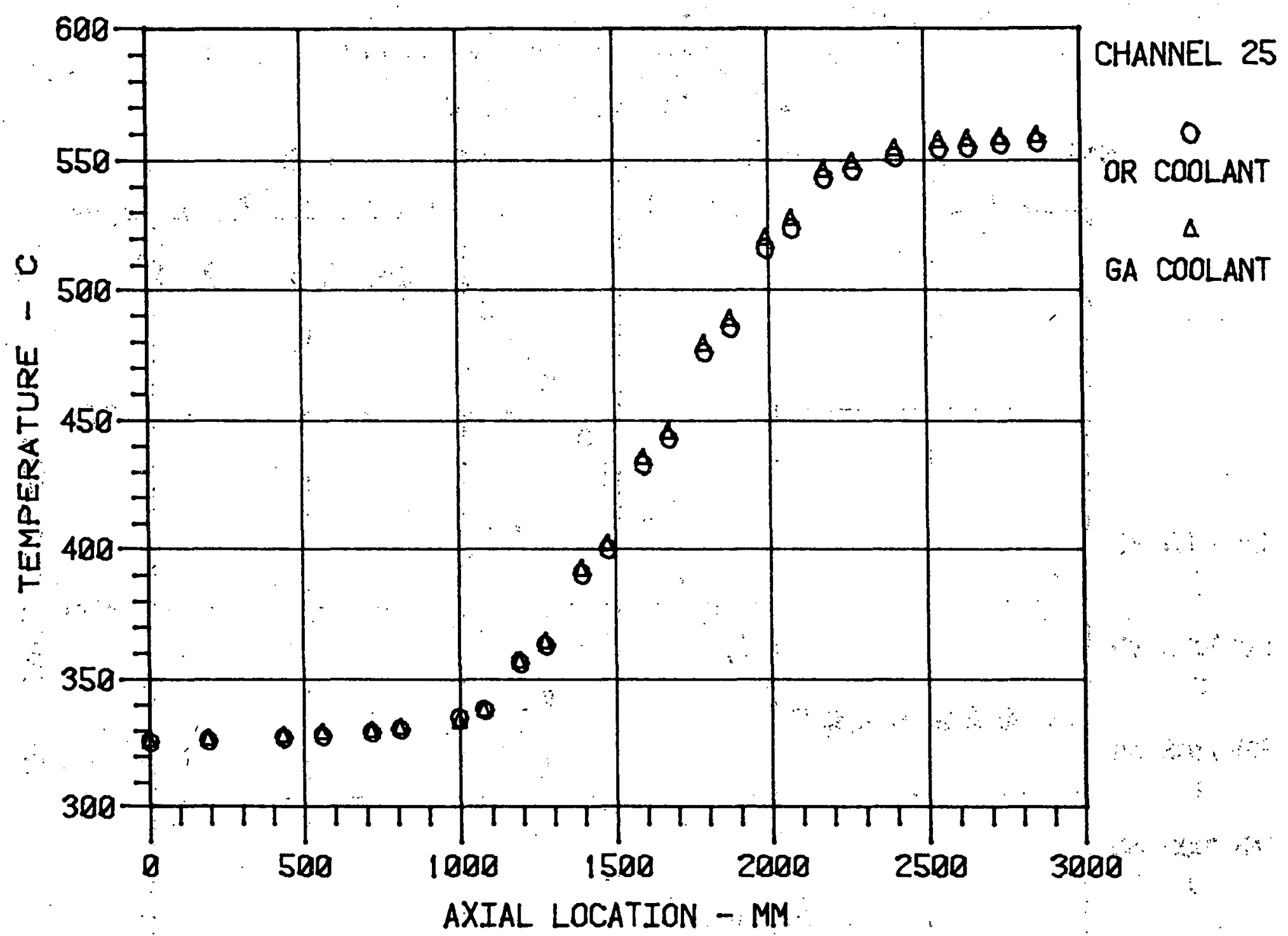

Figure 13. Comparison of COBRA ${ }^{\star} G C F R$ and COBRA/CFTL Results - Test 2: Coolant Temperatures in Channel Next to Hanger Rod. 


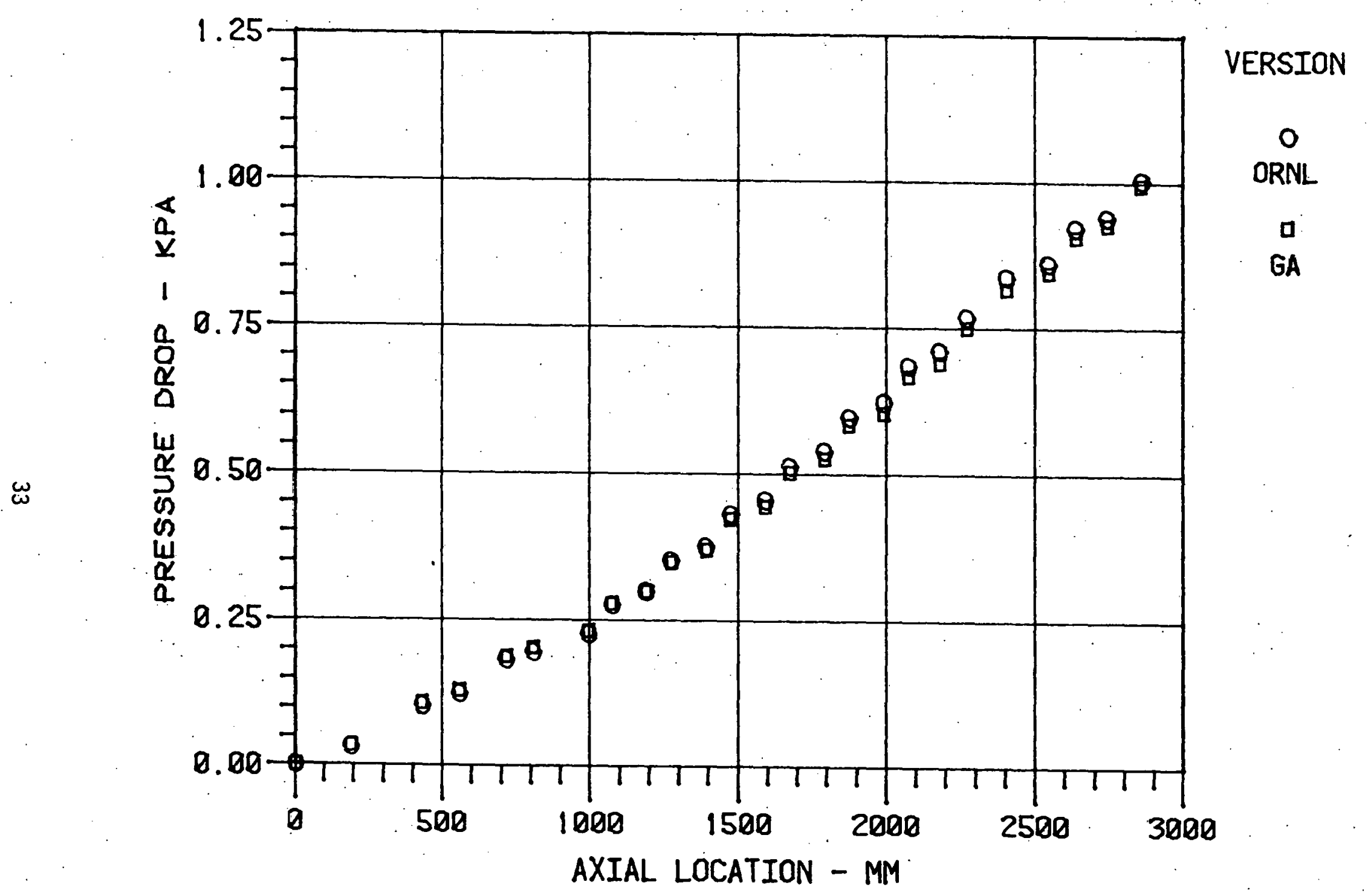

Figure 14. Comparison of COBRA*GCFR and COBRA/CFTL Results - Test 3: Pressure Drop. 


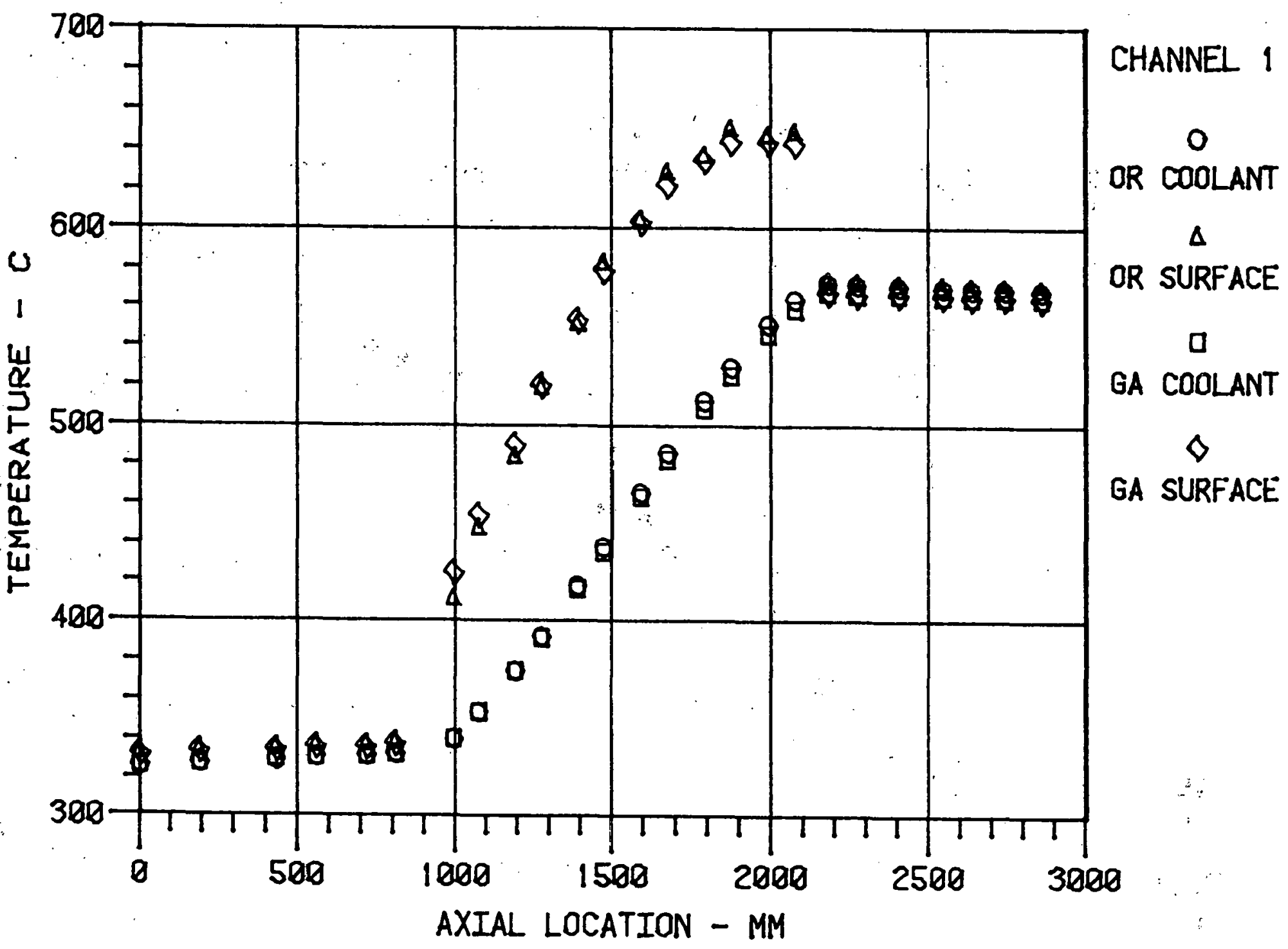

Figure 15. Comparison of COBRA*GCFR and COBRA/CFTL Results - Test 3: Coolant and Rod Surface Temperatures in Interior Channel. 


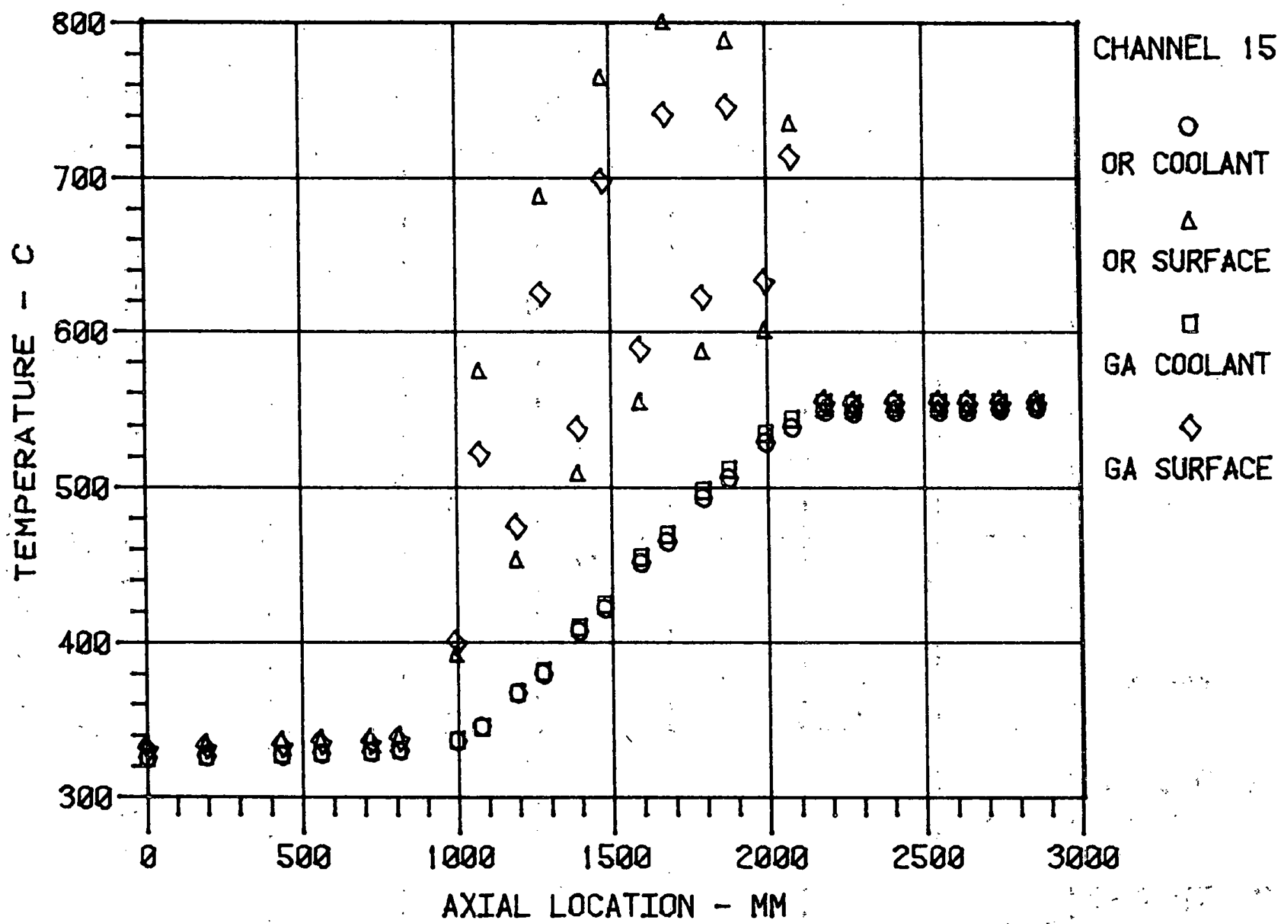

Figure 16. Comparison of COBRA*GCFR and COBRA/CFTL Results - Test 3: Coolant and Rod Surface Temperatures in Wall Channel. 


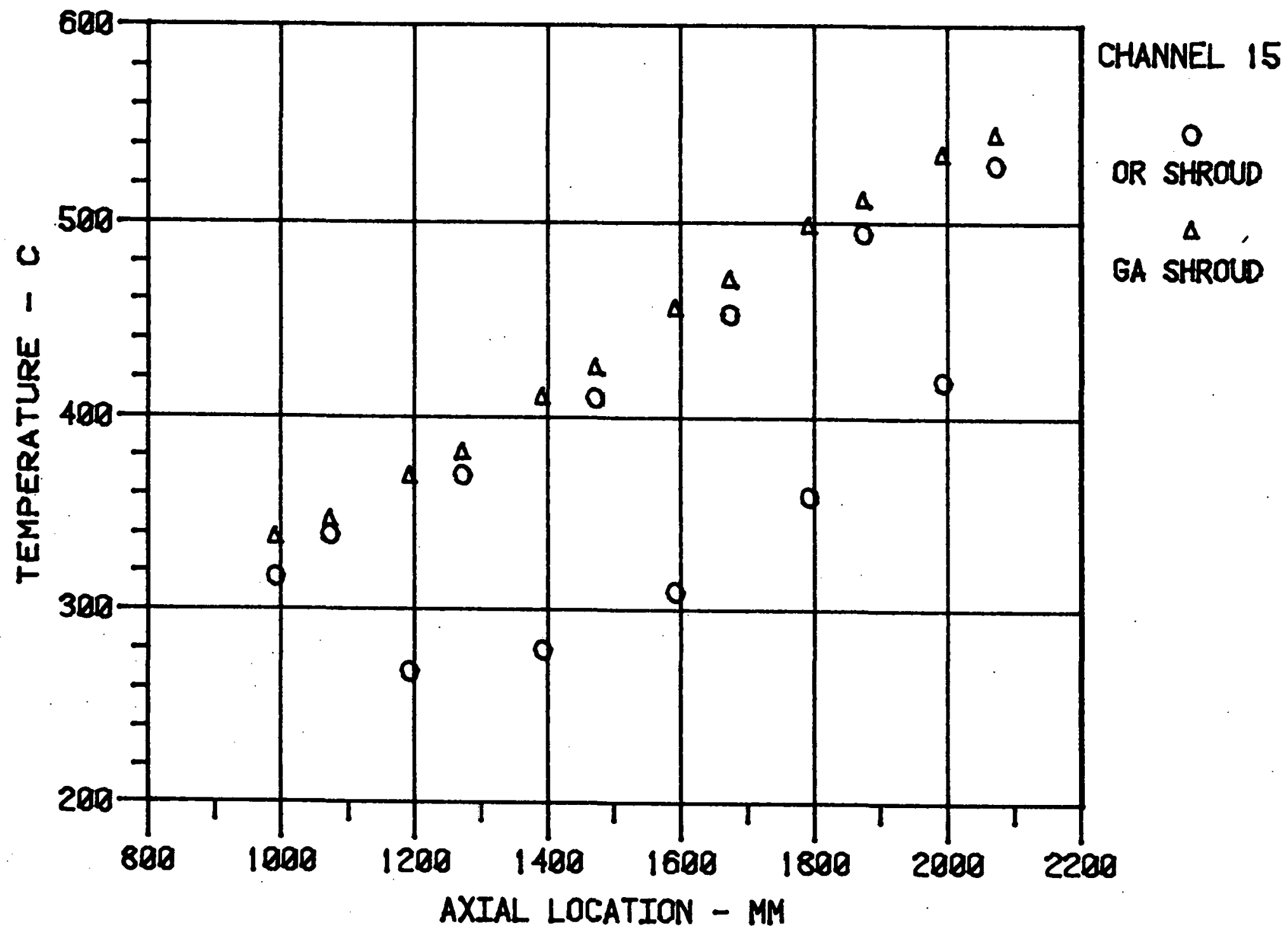

Figure 17. Comparison of COBRA ${ }^{*}$ CCFR and COBRA/CFTL Results - Test 3: Shroud Temperatures in Wall Channel. 


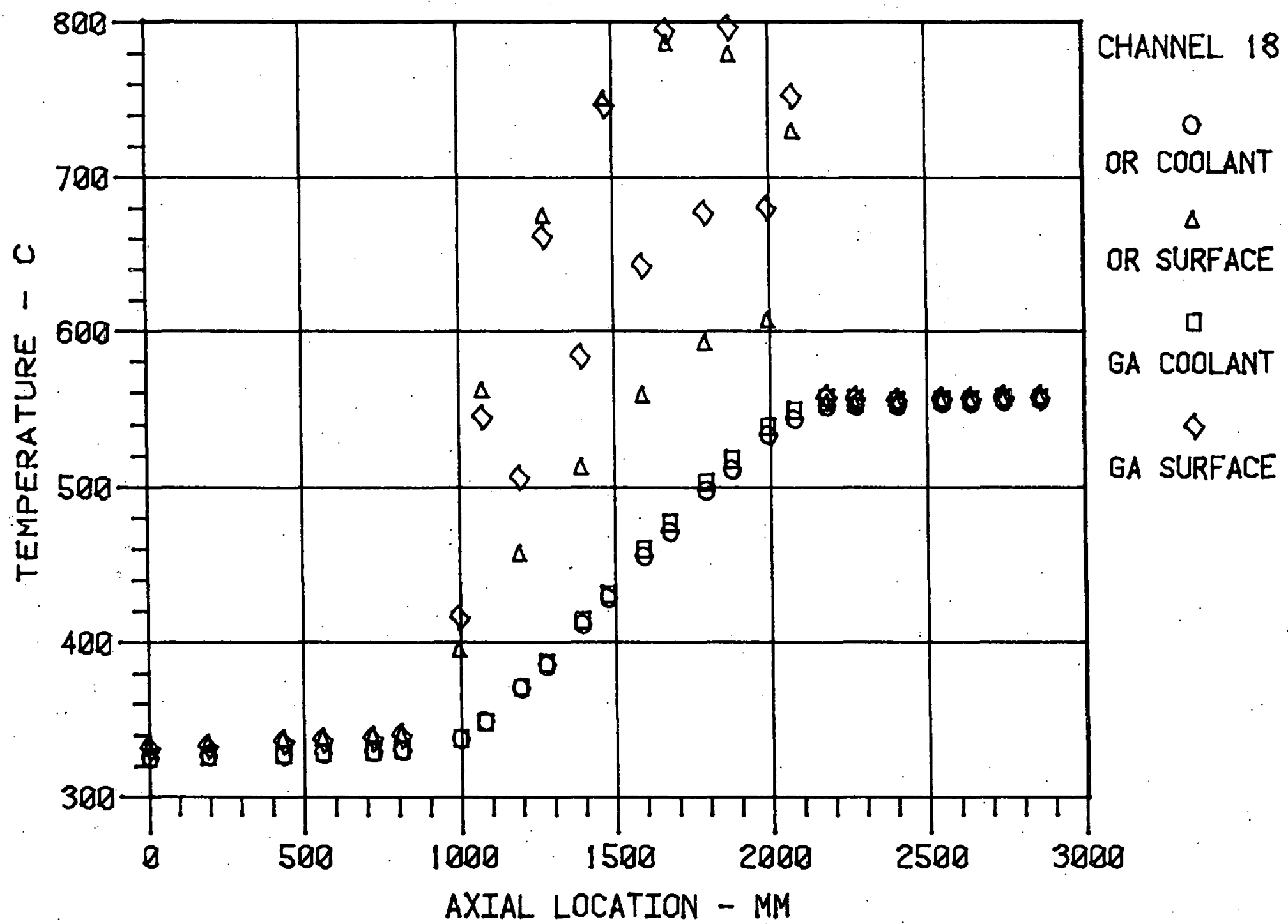

Figure 18. Comparison of COBRA*GCFR and COBRA/CFTL Results - Test 3: Coclant and Rod Surface Temperatures in Corner. Channel. 


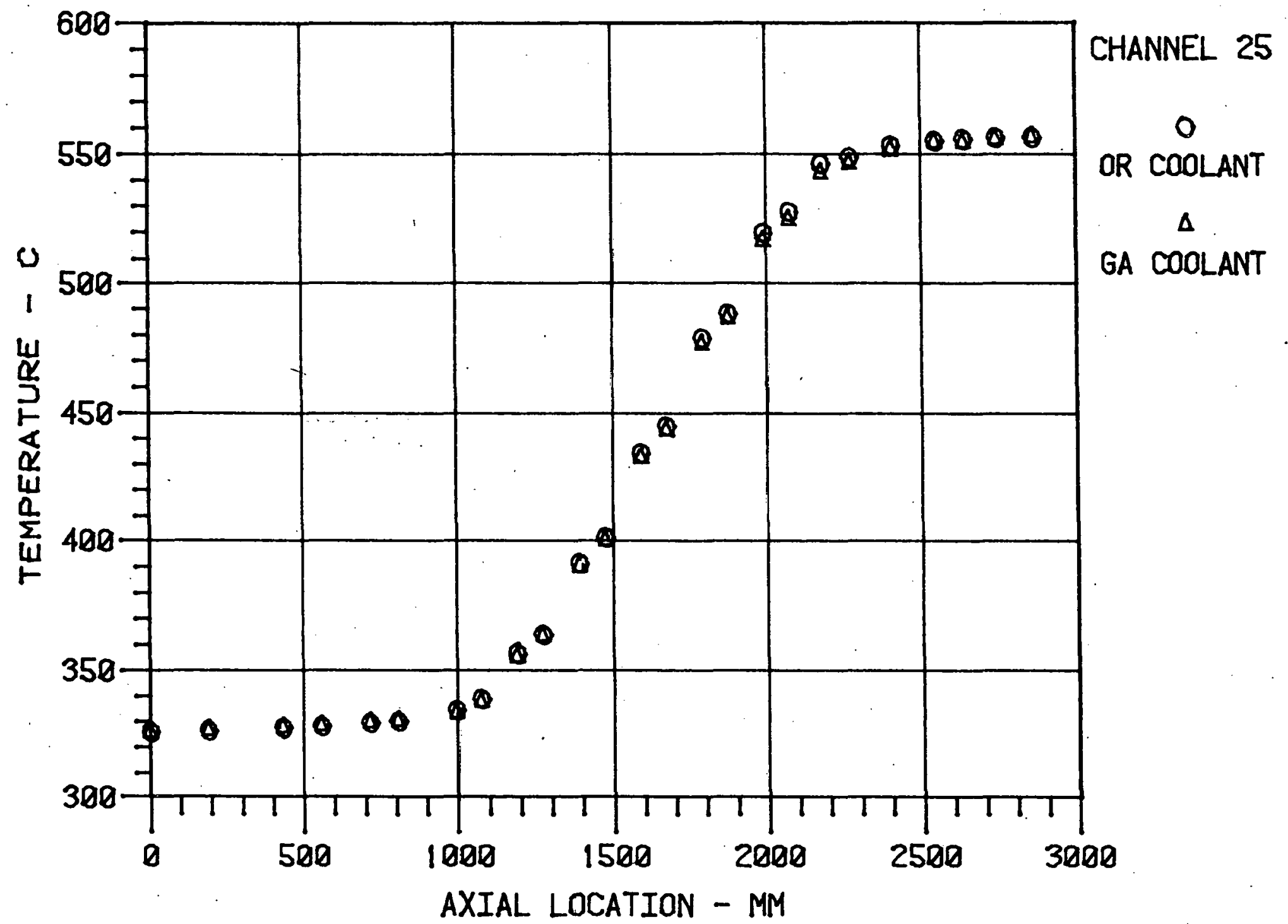

Figure 19. Comparison of COBRA $+G C F R$ and COBRA/CFTL Results - Test 3: Coolant Temperature; in Channel Next to Hanger Rod. 
APPENDIX A

INPUT DATA CARD DESCRIPTIONS FOR COBRA/CFTL CODE 


\begin{tabular}{|l|l|}
\hline WORD & 1 \\
\hline COLUMN & $1-5$ \\
\hline FORMAT & I 5 \\
\hline & Time Limit allowed for problem \\
OA & Default $=1000$, if MAXT read in as zero. \\
\hline SYMBOL & MAXT \\
\hline
\end{tabular}

\begin{tabular}{|l|c|c|}
\hline WORD & 1 & 2 \\
\hline COLUMN & $1-5$ & $6-71$ \\
\hline FORMAT & I 5 & 16 A 4, A 2 \\
\hline \multirow{2}{*}{} & $\begin{array}{l}\text { Read case number } \\
\text { if }<1, \text { program stop }\end{array}$ & $\begin{array}{l}\text { Output text for problem } \\
\text { identification }\end{array}$ \\
\hline OB & & \\
\hline SYMBOL & KASE & TEXT \\
\hline
\end{tabular}

\begin{tabular}{|c|c|c|c|}
\hline WORD & 1 & 2 & 3 \\
\hline COLUMN & $1-5$ & $6-10$ & $11-20$ \\
\hline FORMAT & I 5 & I 5 & $5 X, I 5$ \\
\hline$I A$ & $\begin{array}{l}\text { Group number } \\
(=1)\end{array}$ & $\begin{array}{l}\text { Number of values } \\
\text { in property table. }\end{array}$ & $\begin{array}{l}\text { Property range } \\
=0 \text {, calculates NPRQP properties from (inlet } \\
\text { temperature }-1 \text { ) to } 750^{\circ} \mathrm{C} \\
=1 \text {, calculates NPRQP properties over temper- } \\
\text { ature specified in DTMAX (Card } 1 B \text { ) }\end{array}$ \\
\hline SYMBOL & NGRQUP & $N T:=N P R \emptyset P$ & N3 \\
\hline
\end{tabular}

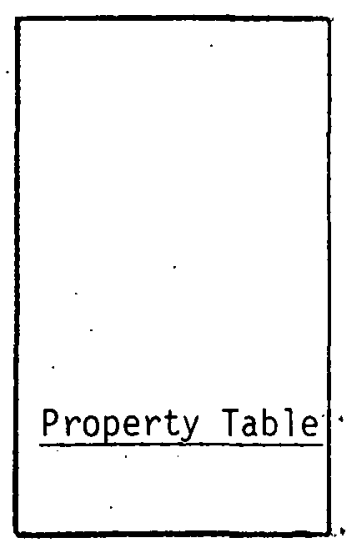

Case Control 
CØBRA/CFTL INPUT

\begin{tabular}{|c|c|}
\hline WORD & 1 \\
\hline COLUMN & $1-5$ \\
\hline FORMAT & F5.3 \\
\hline 18 & $\begin{array}{l}\text { Temperature range over which the } \\
\text { properties are to be calculated. }\end{array}$ \\
\hline SYMBOL & DTMAX \\
\hline
\end{tabular}

Read if

$\mathrm{N} 3=1$

\begin{tabular}{|c|c|c|}
\hline WORD & 1 & 2 \\
\hline COLUMN & $7-5$ & $6-30$ \\
\hline FORMAT & I 5 & $20 x, I 5$ \\
\hline $2 A$ & $\begin{array}{l}\text { Group Number } \\
(=2)\end{array}$ & $\begin{array}{l}\text { Laminar friction factar } \\
=0 \text {, no laminar correlation } \\
=1 \text {, read in up to NFT sets of laminar friction factor } \\
=2 \text {, Correlation constants } \\
\end{array}$ \\
\hline SYMBOL & NGRØUP & N5 $=$ LAMNF \\
\hline
\end{tabular}

Friction and Nusselt

Correlations

\begin{tabular}{|c|c|c|c|}
\hline WORD & 3 & 4 & 5 \\
\hline COLUMN & $31-35$ & $36-40$ & $41-45$ \\
\hline FORMAT & I 5 & I 5 & I 5 \\
\hline $\begin{array}{l}2 \mathrm{~A} \\
\text { (Cont. })\end{array}$ & $\begin{array}{l}\text { Rod-to-coolant single phase heat transfer } \\
=0 \text {, use Dittus-Boelter correlation } \\
>0 \text {, read a user-supplied Nusselt } \\
\text { correlation (Card 2D) }\end{array}$ & $\begin{array}{l}\text { Friction and heat } \\
\text { transfer correla- } \\
\text { tions (see below) }\end{array}$ & $\begin{array}{l}\text { Number of friction or } \\
\text { Stanton correlation types } \\
\text { to read if } \mathrm{NFH}>0 \text {. }\end{array}$ \\
\hline SYMBOL & N6 & $\mathrm{N} 7=\mathrm{NFH}$ & $N 8=N F T$ \\
\hline
\end{tabular}

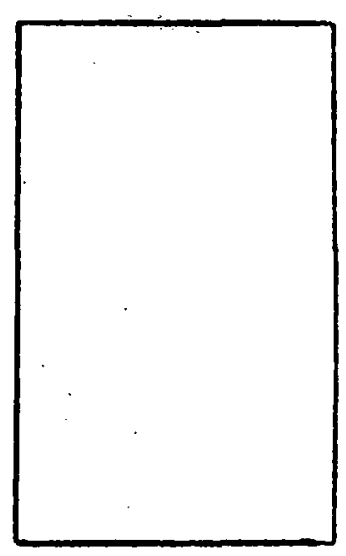




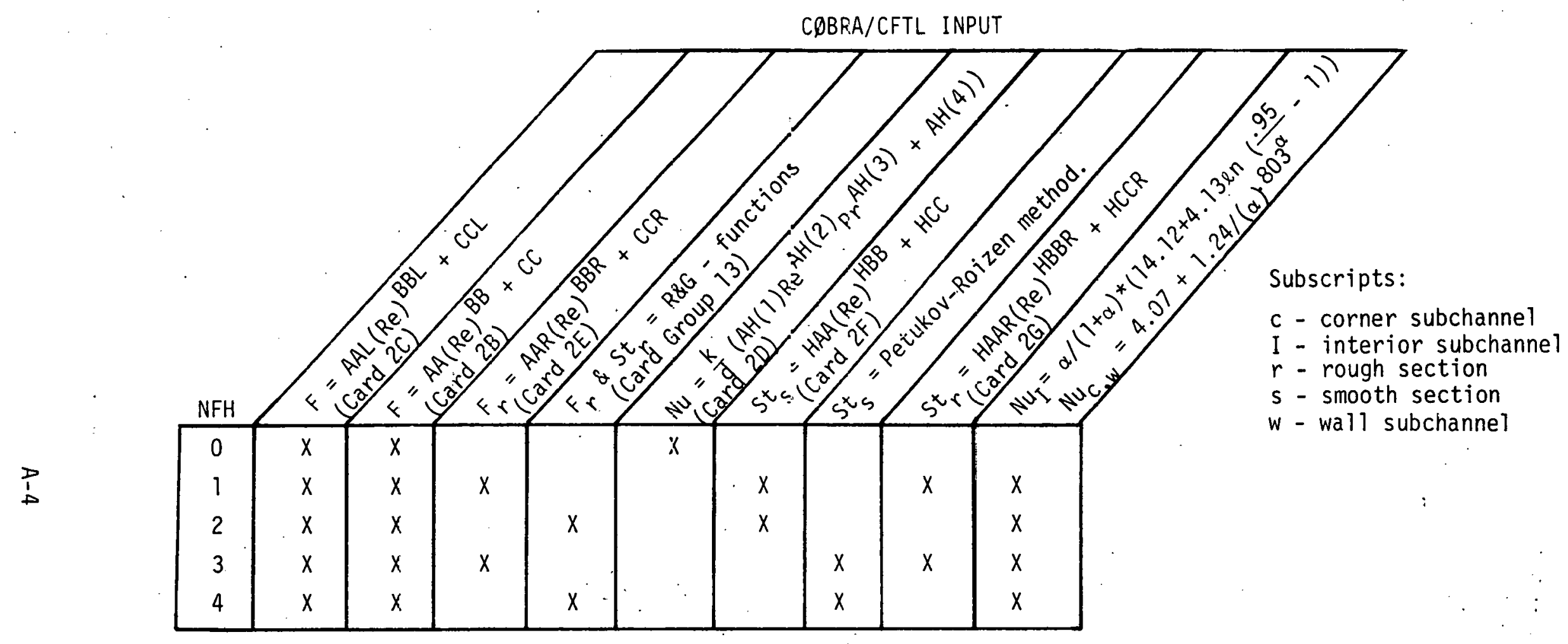

For LAMNF $>0, \quad F=$ Maximum of $\left\{A A L(R e)^{B B L}+C C L\right.$

$\{F$ for turbulert flow 


\begin{tabular}{|c|c|}
\hline WOF:D & $1-12$ \\
\hline COLUMN & $1-60$ \\
\hline FOFMAT & $6 \mathrm{E} 10.5$ \\
\hline $2 B^{*}$ & $\begin{array}{l}\text { Turbulent friction factor correlation coefficients. If } N F H>0 \text {, this is for the axially } \\
\text { smooth section; otherwise, it is used for the entire axial length. } \\
\qquad f=A A(\operatorname{Re})^{B B}+C C \quad[I=\text { subchannel type }]\end{array}$ \\
\hline SYMBOL. & $A A(I), B B(I), C C(I), I=1, N F T$ \\
\hline
\end{tabular}

\begin{tabular}{|c|c|}
\hline WORD & $1-12$ \\
\hline COLUMN & $1-60$ \\
\hline FORMAT & $6 \mathrm{E} 10.5$ \\
\hline $2 \mathrm{C}$ & $\begin{array}{l}\text { Laminar friction factor correlation coefficients } \\
\qquad f_{L}=A A L(R e)^{B B L}+C C L\end{array}$ \\
\hline SYMBOL & $\operatorname{AAL}(I), \operatorname{BBL}(I), \operatorname{CCL}(I), I=1, N F T$ \\
\hline
\end{tabular}

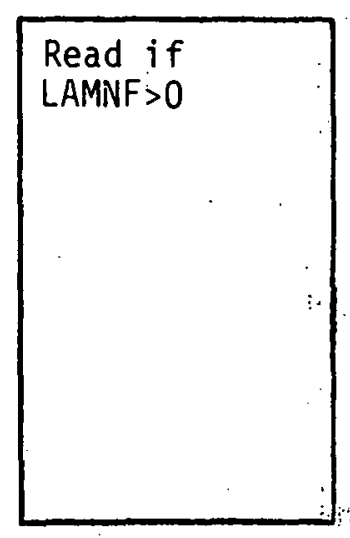

\begin{tabular}{|l|c|}
\hline WORD & $1-4$ \\
\hline COLUMN & $1-20$ \\
\hline FORMAT & 4 F5.3 \\
\hline & Single phase Nusselt number correlation coefficients \\
& $h=\frac{k}{D}\left[A H(1) \operatorname{Re}^{\mathrm{AH}(2)} \mathrm{Pr} A H(3)+\mathrm{AH}(4)\right]$ \\
& $\vdots$ \\
\hline SYMBOL & $\mathrm{AH}(\mathrm{I}), \mathrm{I}=1,4$ \\
\hline
\end{tabular}

$\underset{\substack{\text { Read } \\ \text { if }}}{ }$

*Put this card here after card 2D if $L A M N F>0$ and $N F H>0$. Coefficients are for the smooth section only. 


\begin{tabular}{|c|c|}
\hline WORD & $1-6$ \\
\hline COLUMN & $1-60$ \\
\hline FORMAT & $6 \mathrm{E} 10.5$ \\
\hline$\vdots$ & $\begin{array}{l}\text { Friction factor correlation coefficients for axially rough section. } \\
\qquad F_{R}=A A R(R e)^{B B R}+C C R \quad(I=\text { subcharnel type })\end{array}$ \\
\hline SYMBOL & $\operatorname{AAR}(I), \operatorname{BBR}(I), \operatorname{CCR}(I), I=1, N F T$ \\
\hline
\end{tabular}

\begin{tabular}{|c|c|}
\hline WORD & $1-6$ \\
\hline COLUMN & $1-60$ \\
\hline FORMAT & $6 \mathrm{E} 10.5$ \\
\hline $2 \mathrm{~F}$ & $\begin{array}{l}\text { Stanton number correlation coefficients for axially smooth section } \\
\qquad S t_{S}=H A A(R e)^{H B B}+H C C \quad(I=\text { subchannel type })\end{array}$ \\
\hline SYMBOL & $\operatorname{HAA}(I), \operatorname{HBB}(I), \operatorname{HCC}(I), I=1, N F \pi$ \\
\hline
\end{tabular}

\begin{tabular}{|c|}
\hline Read if \\
$N F H=1,2$
\end{tabular}

\begin{tabular}{|l|c|}
\hline WORD & $1-6$ \\
\hline COLUMN & $1-60$ \\
\hline FORMAT & $6 E 10.5$ \\
\hline & Stanton number correlation coefficients for axially rough section \\
& $S t_{R}=\operatorname{HAAR}(\mathrm{Re})^{\text {HBBR }}+\mathrm{HCCR} \quad(\mathrm{I}=$ subchannel type $)$ \\
\hline SYMBOL & $\operatorname{HAAR}(\mathrm{I}), \mathrm{HBBR}(\mathrm{I}), \mathrm{HCCR}(\mathrm{I}), \mathrm{I}=1, \mathrm{NFT}$ \\
\hline
\end{tabular}




\begin{tabular}{|c|c|c|c|c|}
\hline WORD & .1 & 2 & 3 & 4 \\
\hline COLUMN & $1-5$ & $6-10$ & $11-15$ & $16-20$ \\
\hline FORMAT & I 5 & I 5 & 15 & I 5 \\
\hline $3 \mathrm{~A}$ & $\begin{array}{l}\text { Group } \\
\text { Number } \\
(=3)\end{array}$ & $\begin{array}{l}\text { No: of entries in } \\
\text { axial heat flux } \\
\text { table: }\end{array}$ & $\begin{array}{l}\text { Radiation heat transfer model } \\
=0 \text {, no radiation } \\
=1 \text {, radiation }\end{array}$ & $\begin{array}{l}\text { Logical unit for reading } \\
\text { Hottel's radiation } \\
\text { exchange factors when } \\
\text { IRAD }=1\end{array}$ \\
\hline SYMEOL & NGRQUP & $N I=N A X$ & $\mathrm{~N} 2=\mathrm{IRAD}$ & N3 \\
\hline
\end{tabular}

Axial Heat

\begin{tabular}{|c|c|c|c|}
\hline WORL & \multicolumn{3}{|c|}{$1-12$} \\
\hline COLUMN & \multicolumn{3}{|c|}{$1-60$} \\
\hline FORMAT & \multicolumn{3}{|c|}{$12 F 5.3$} \\
\hline $3 B$ & $\begin{array}{l}\text { Axial heat flux table } \\
Y=\text { relative position }(X / L) \\
\text { AXIAL }=\text { relative heat flux (local/average) }\end{array}$ & 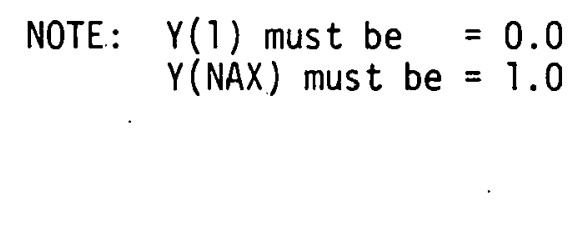 & $\therefore$ \\
\hline SYMBOL & $Y: I), \operatorname{AXIAL}(I), I=I, \operatorname{NAX}$ & & \\
\hline
\end{tabular}

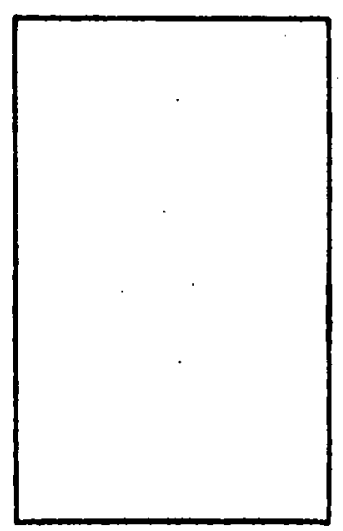

\begin{tabular}{|c|c|c|c|c|c|}
\hline WORD & 1 & 2 & 3 & 4 & 5 \\
\hline COLUMN & $1-5$ & $6-10$ & $11-15$ & $16-20$ & $27-25$ \\
\hline FORMAT & 15 & I 5 & I 5 & $:$ I 5 & I 5 \\
\hline $4 A$ & $\begin{array}{l}\text { Group } \\
\text { number } \\
(=4)\end{array}$ & $\begin{array}{l}\text { No. of subchannel } \\
\text { data cards to be } \\
\text { read }\end{array}$ & $\begin{array}{l}\text { Total no. } \\
\text { of sub-: } \\
\text { channels }\end{array}$ & $\begin{array}{l}=0 \text {, no directed crossflows } \\
>0 \text {, the number of gaps for } \\
\text { which directed cross- } \\
\text { flows are specified } \\
\text { (Card 4D). }\end{array}$ & $\begin{array}{l}=0, \text { no thermal } \\
\text { connections } \\
>0, \text { number of } \\
\text { thermal } \\
\text { connections } \\
\text { (Card 4C) }\end{array}$ \\
\hline SYMBILL & NGRØUP & N1 & $\mathrm{N} 2=\mathrm{NCHANL}$ & N3 $=N S$ & $N 4=N W K$ \\
\hline
\end{tabular}

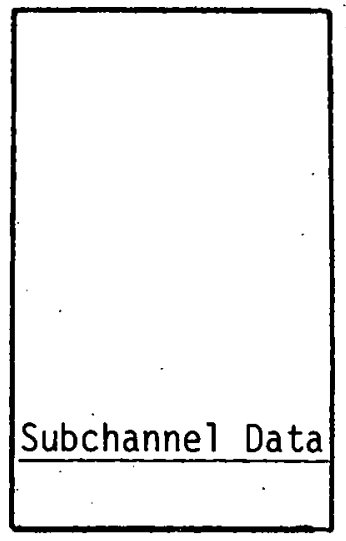


CØBRA/CFTL INPUT

\begin{tabular}{|l|c|c|}
\hline WORD & 6 & 7 \\
\hline COLUMN & $26-30$ & $31-35$ \\
\hline FORMAT & I 5 & \multicolumn{1}{|c|}{ I 5 } \\
\hline $\begin{array}{l}\text { AA } \\
\text { Cont.) }\end{array}$ & $\begin{array}{l}\text { Logical unit for reading } \\
\text { Subchannel geometry data } \\
=0, \text { read from cards } \\
>0, \text { number of logical unit }\end{array}$ & $\begin{array}{l}\text { Logical unit for reading } \\
\text { directed crossflow data } \\
=0, \text { read from cards } \\
>0 \text {, number of logical unit }\end{array}$ \\
\hline SYMBOL & N5 & N6 \\
\hline
\end{tabular}

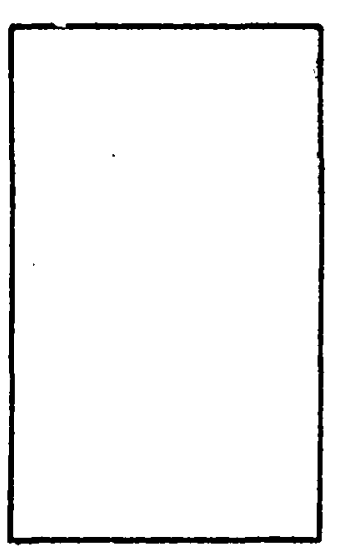

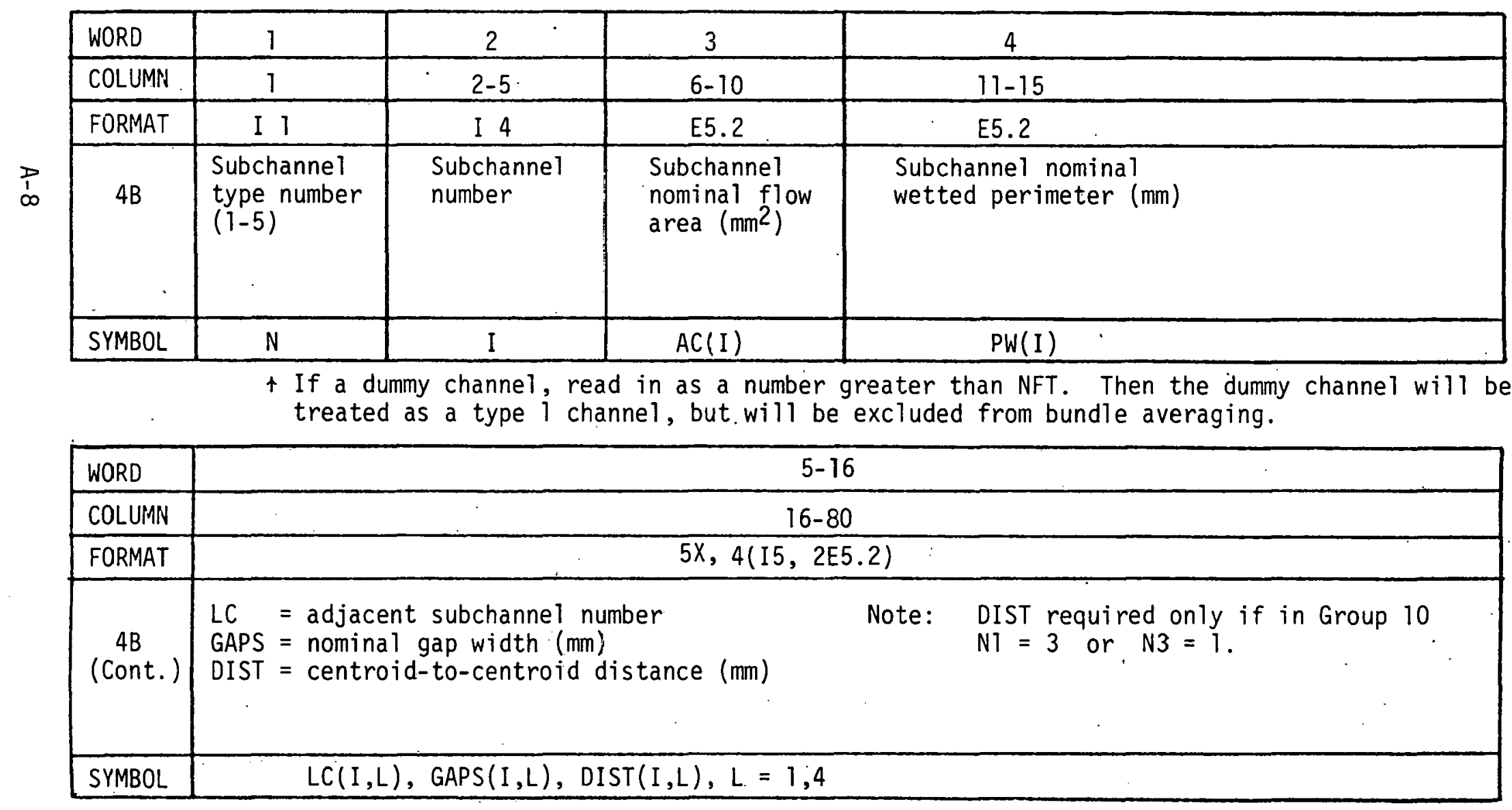
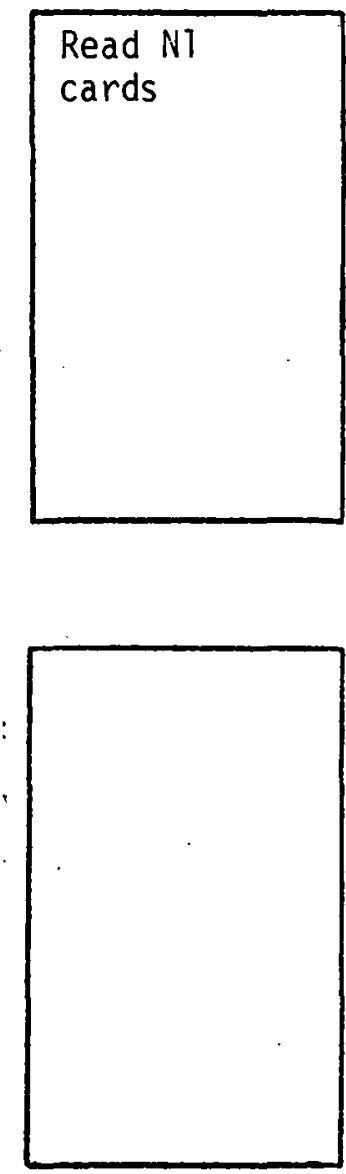
CØBRA/CFTL INPUT

\begin{tabular}{|c|c|c|c|c|c|}
\hline WORLI & 1 & 2 & 3 & 4 & 5 \\
\hline COLUMN & $3-5$ & $6-10$ & $11-15$ & $16-20$ & $21-25$ \\
\hline FORMAT & I 5 & E5.2 & $E 5.2$ & I 5 & $\mathrm{E} 5.2$ \\
\hline $4 C$ & $\begin{array}{l}\text { Therma } 1 \\
\text { connection } \\
\text { number. }\end{array}$ & $\begin{array}{l}\text { Wall heat } \\
\text { capacity } \\
\text { parameter } \\
\left(K_{1} J / m^{2}-K\right)\end{array}$ & $\begin{array}{l}\text { Width of wall } \\
{\left[\begin{array}{c}\text { heat conduction } \\
\text { area }=\text { WIDTH*DX }\end{array}\right] .} \\
(\mathrm{mm})\end{array}$ & $\begin{array}{l}\text { Subchannel no. } \\
\text { adjacent to } \\
\text { wal? }\end{array}$ & $\begin{array}{l}\text { Conductive resistance of } \\
\text { wall associated with IKW } \\
\text { subchannel } \\
\qquad\left(\frac{\mathrm{m}^{2}-\mathrm{K}}{\mathrm{kW}}\right) .\end{array}$ \\
\hline SYMBOL & $K W$ & RH $\emptyset \mathrm{LCP}$ & WIDTH & $I K W$ & $\operatorname{RWALL}(1, \mathrm{KW})$ \\
\hline
\end{tabular}

\begin{tabular}{|c|c|c|}
\hline WORD & 6 & 7 \\
\hline COLUMN & $26-30$ & $31-35$ \\
\hline FORMAT & I 5 & E5.2 \\
\hline $\begin{array}{c}4 C \\
\text { (Cont.) }\end{array}$ & $\begin{array}{l}\text { Subchannel number } \\
\text { adjacent to wall }\end{array}$ & $\begin{array}{l}\text { Conductive resistance of wall } \\
\text { associated with JKW subchannel } \\
\qquad\left(\frac{\mathrm{m}^{2}-\mathrm{K}}{\mathrm{kW}}\right)\end{array}$ \\
\hline SYMBOLL & JKW & $\operatorname{RWALL}(2 ; \mathrm{KW})$ \\
\hline
\end{tabular}

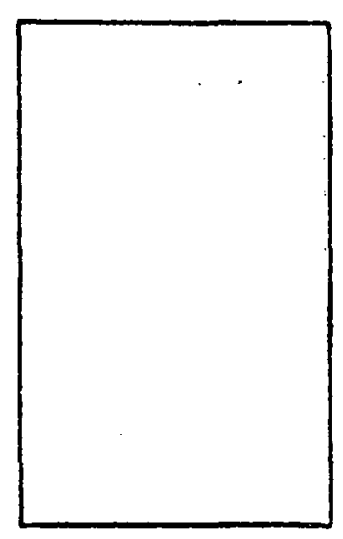

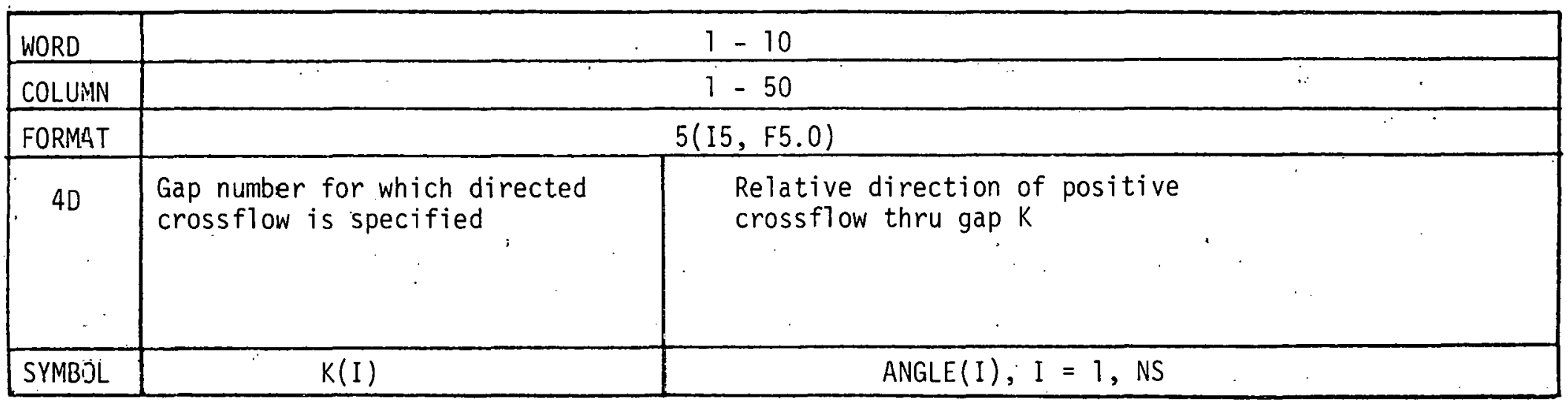

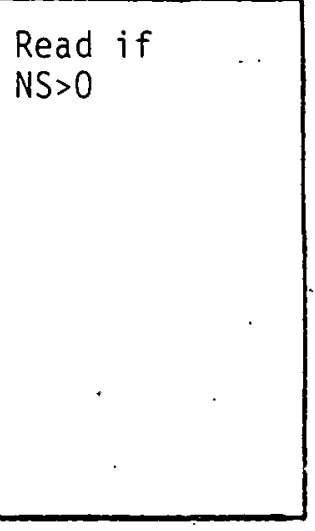


CØBRA/CFTL INPUT

\begin{tabular}{|c|c|c|c|c|}
\hline WORD. & 1 & 2 & 3 & 4 \\
\hline COLUMN & $1-5$ & $6-10$ & $11-15$ & $16-20$ \\
\hline FORMAT & I 5 & I. 5 & 15 & 15 \\
\hline $5 A$ & $\begin{array}{l}\text { Group number } \\
(=5)\end{array}$ & $\begin{array}{l}\text { Number of subchannels } \\
\text { with area variations }\end{array}$ & $\begin{array}{l}\text { Number of axial locations } \\
\text { for area variations }\end{array}$ & $\begin{array}{l}\text { Number of iterations } \\
\text { for gradual insertion } \\
\text { of area variations } \\
\text { (Default }=1)\end{array}$ \\
\hline SYMBOL & NGRØUP & $\mathrm{N} I=\mathrm{NAFACT}$ & N2-NAXL & $N 3=N A R A M P$ \\
\hline
\end{tabular}

Area variation

\begin{tabular}{|c|c|}
\hline WORD & $1--2$ \\
\hline COLUMN & $1-60$ \\
\hline FORMAT & $12 F 5.2$ \\
\hline $5 B$ & $\begin{array}{l}\text { Relative axial locations }(X / L) \text { where subchannel } \\
\text { area variations are being specified }\end{array}$ \\
\hline SYMBOL & $\operatorname{AXL}(I), I=1, \operatorname{NAXL}$ \\
\hline
\end{tabular}

\begin{tabular}{|c|c|c|}
\hline WORD & 1 & $1-12$ \\
\hline COLUMMN & $1-5$ & $1-60$ \\
\hline FORMAT & 15 & $12 F 5.3$ \\
\hline $\begin{array}{l}5 C \\
5 D\end{array}$ & Subchannel number & $\begin{array}{l}\text { Relative subchannel area }\left(A_{i} / A_{\text {nominall }}\right) \text { at each } \\
\text { axial location }(A L)\end{array}$ \\
\hline SYMBOL & I & AFACT \\
\hline
\end{tabular}

Read if

$N A X L>0$

Read NAXL

values for

each sub-

channel. 
COBRA/CFTL INPUT

\begin{tabular}{|l|c|c|c|}
\hline WORD & 1 & 2 & 3 \\
\hline COLIJMN & $1-5$ & $6-10$ & $11-15$ \\
\hline FORMAT & I 5 & I 5 & I 5 \\
\hline $6 A$ & $\begin{array}{l}\text { Group number } \\
(=6)\end{array}$ & $\begin{array}{l}\text { Number of gaps for which gap variation } \\
\text { tables are to be read }\end{array}$ & $\begin{array}{l}\text { Number of axial locations for } \\
\text { gap variation }\end{array}$ \\
\hline SYMBOL & NGR & N1 $=$ NGAPS & N2 $=$ NGXL \\
\hline
\end{tabular}

Gap Size

Variation

\begin{tabular}{|c|c|}
\hline WORD & $1-12$ \\
\hline COLUMN & $1-60$ \\
\hline FORMAT & $12 F 5.3$ \\
\hline $6 \mathrm{~B}$ & $\begin{array}{l}\text { Relative axial locations }(x / L) \text { where gap variations } \\
\text { are beir:g specified. }\end{array}$ \\
\hline SYMEOL & $\operatorname{GAPXL}(I), I=1, N G X L$ \\
\hline
\end{tabular}

Read if

$N G X L>0$

Read if

$N G X L>0$

Read NGXL

values for

each gap.

\begin{tabular}{|c|c|c|}
\hline $\begin{array}{l}6 C \\
60\end{array}$ & Gap number & Relative gap length (GAP ${ }_{i} /$ GAP nominal) at each axial location (GAPXL) \\
\hline SYMBOL & $k$ & GFACT \\
\hline
\end{tabular}


CØBRA $/ C F T L$ INPUT

\begin{tabular}{|c|c|c|c|c|c|}
\hline WORD & 1 & 2 & 3 & 4 & 5 \\
\hline COLUMN & $1-5$ & $6-10$ & $17-15$ & $\cdot 16-20$ & $21-25$ \\
\hline FORMAT & 15 & 15 & I 5 & I 5 & I 5 \\
\hline $7 \mathrm{~A}$ & $\begin{array}{l}\text { Group } \\
\text { number } \\
(=7)\end{array}$ & $\begin{array}{l}=1 \text {, wire wrap input only } \\
=2 \text {, grid spacer input only } \\
=3 \text {, both wire wrap and grid } \\
\text { spacer input (loss } \\
=0, \text { noefficients) } \\
\text { no wire wrap or spacers }\end{array}$ & $\begin{array}{l}\text { No. of gaps for } \\
\text { which wire wrap } \\
\text { crossing data } \\
\text { is supplied }\end{array}$ & $\begin{array}{l}\text { No. of axial } \\
\text { locations for } \\
\text { grid spacers }\end{array}$ & $\begin{array}{l}\text { No. of grid type } \\
\text { for which data } \\
\text { will be supplied }\end{array}$ \\
\hline SYMBOL & NGRØUP & $N]=J 6$ & $\mathrm{~N} 2$ & $N 3=N G R I D$ & $\mathrm{~N} 4=\mathrm{NGRIDT}$ \\
\hline
\end{tabular}

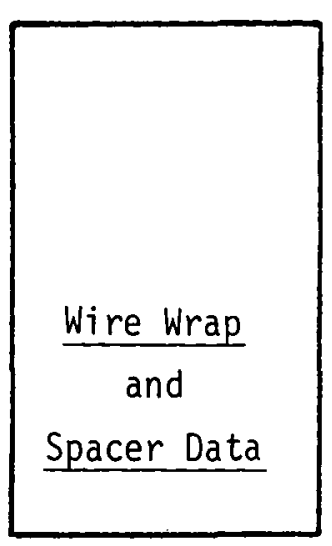

\begin{tabular}{|l|c|c|c|}
\hline WORD & 6 & 7 & 8 \\
\hline COLUMN & $26-30$ & $31-35$ & $36-40$ \\
\hline FORMAT & I 5 & I 5 & I 5 \\
\hline $\begin{array}{l}7 A \\
\text { (Cont.) }\end{array}$ & $\begin{array}{l}\text { Number of iterations over which } \\
\text { grid loss terms are to be } \\
\text { ramped into the solution. } \\
\text { [Default = 1] }\end{array}$ & $\begin{array}{l}\text { Logical unit for reading wrap } \\
\text { crossing data and wrap } \\
\text { inventory } \\
=0, \text { read from cards } \\
>0, \text { number of logical unit }\end{array}$ & $\begin{array}{l}\text { Number of channels for } \\
\text { which wire wrapping } \\
\text { information is to be } \\
\text { read } \\
\text { (Default }\end{array}$ \\
\hline SYMBOL & N5 = NRAMP & N6 & N7 \\
\hline
\end{tabular}

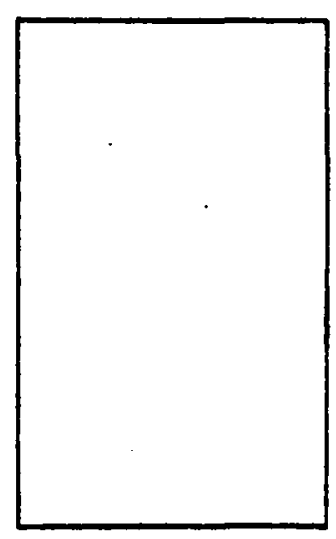

\begin{tabular}{|l|c|c|c|}
\hline WORD & 1 & 2 & 3 \\
\hline COLUMN & $1-10$ & $11-20$ & $21-30$ \\
\hline FORMAT & E10.5 & E10.5 & E10.5 \\
\hline 7B & $\begin{array}{l}\text { Wire wrap pitch } \\
(\mathrm{mm})\end{array}$ & $\begin{array}{l}\text { Rod or cladding outer diameter } \\
(\mathrm{mm})\end{array}$ & $\begin{array}{c}\text { Wire wrap diameter } \\
(\mathrm{mm})\end{array}$ \\
\hline SYMBOL & PITCH & DIA & THICK \\
\hline
\end{tabular}

Read if

$j 6=1,3$

Wire Wrap Specifications 
CØBRA/CFTL INPUT

\begin{tabular}{|l|c|c|c|}
\hline WORD & 1 & 2 & $3 \& 4$ \\
\hline COLUMN & $1-5$ & $6-10$ & $11-20$ \\
\hline FORMAT & $I 5$ & E5.2 & $2 E 5.2$ \\
\hline TC & Gap number & $\begin{array}{l}\text { Effective fraction of pitch length } \\
\text { for forced cross flow } \\
\text { axial node length }\end{array}$ & $\begin{array}{l}\text { Wire wrap crossing angle-positive if } \\
\text { wire moving from lower to higher } \\
\text { subchannel number } \\
\text { wire wrap pitch }\end{array}$ \\
\hline SYMBOL & $K$ & DUR & XCROSS $(K, L), L=1,2$ \\
\hline
\end{tabular}

Read if

$\mathrm{J} 6=1,3$

Read N2 cards

Wire Wrap

Crossing Data

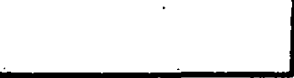

\begin{tabular}{|c|c|}
\hline WORD & $1-10$ \\
\hline COLLIMN & $1-50$ \\
\hline FORMAT & $10 I 5$ \\
\hline 70 & $\begin{array}{l}\text { Number of wires initially in each subchannel (at bundle inlet) } \\
\text { Wire on gap joundary assumed to be in subchannel into which it is proceeding }\end{array}$ \\
\hline SYMEOL & $\operatorname{NWRAPS}(I), I=1, N 7$ \\
\hline
\end{tabular}

Read if
$\mathrm{J} 6=1,3$
Wire
Inventory

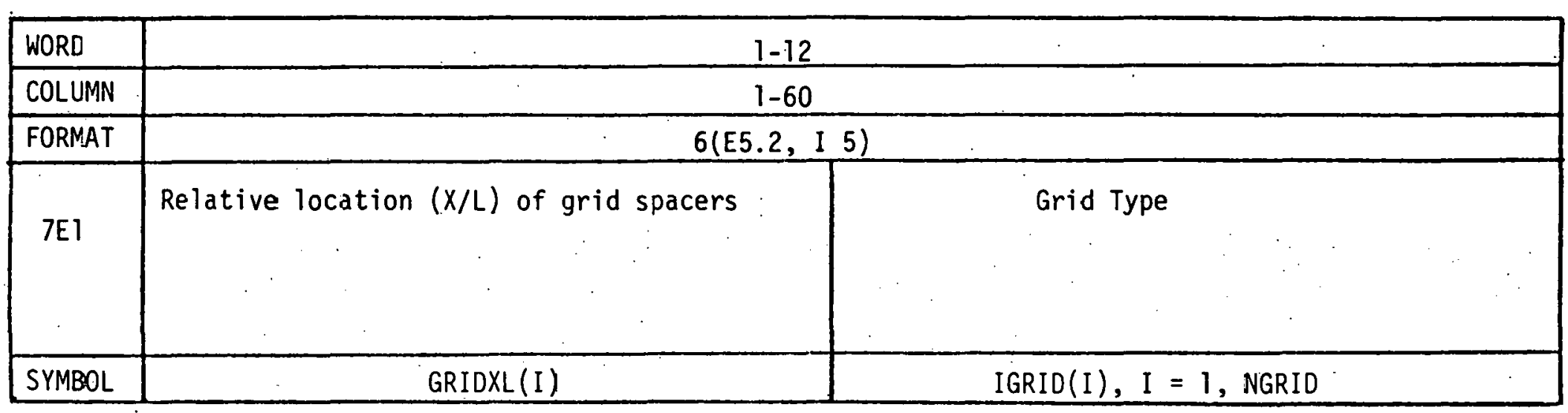

Read if

$\mathrm{J} 6=2,3$

Grid Spacer

Specifications 
CØBRA/CFTL INPUT

\begin{tabular}{|c|c|}
\hline WORD & $1-4$ \\
\hline COLUMN & $1-40$ \\
\hline FORMAT & $4 \mathrm{E} 10.5$ \\
\hline $7 E 2$ & $\begin{array}{l}\text { Equation for spacer loss coefficients } \\
\qquad \operatorname{COEF}=\operatorname{CD}(\mathrm{J}, \mathrm{I}) \star \star \operatorname{CDCl}(\mathrm{I}) \star\left(\operatorname{CDC} 2(\mathrm{I})+\frac{\operatorname{CDC} 3(\mathrm{I})}{\operatorname{Re} \operatorname{CDC}(\mathrm{I})}\right) \\
\text { where } \operatorname{CD}(\mathrm{J}, \mathrm{I}) \text { is read on Card } 7 \mathrm{~F}\end{array}$ \\
\hline SYMBOL & $\operatorname{CDCl}(I), \operatorname{CDC} 2(I), \operatorname{coc} 3(I), \operatorname{CDC} 4(I), I=1$, NGRIDT \\
\hline
\end{tabular}

Read if

$\mathrm{J} 6=2,3$

Read NGRIDT

cards

Read if

$J 6=2,3$

\begin{tabular}{|l|c|}
\hline WORD & 1 \\
\hline COLUMN & $1-10$ \\
\hline FORMAT & E10.5 \\
\hline $7 E 3$ & $\begin{array}{l}\text { Spacer height } \\
\text { (mm) }\end{array}$ \\
\hline SYMBOL & SH \\
\hline
\end{tabular}

Rough spacer coefficient

$Y S T=1+R S C * C D^{2} * F(X)$

Smooth spacer coefficient

YST $=1+S_{S C} \star C D^{2} \star F(X)$

SSC

\begin{tabular}{|c|c|c|c|c|}
\hline WORD & 1 & 2 & 3 & 4 \\
\hline COLUMN & $1-5$ & $6-10$ & $11-15$ & $16-20$ \\
\hline FORMAT & I 5 & $\mathrm{E} 5.2$ & I 5 & $E 5.2$ \\
\hline $7 F$ & Subchannel number & $\begin{array}{l}\text { Solidity for grid type } \\
\text { I in subchannel } \mathrm{J} \\
\left.\text { (A } A_{\text {spacer }} / A_{\text {channel }}\right)\end{array}$ & $\begin{array}{l}\text { Gap associated with } \mathrm{J} \text { thru } \\
\text { which forced diversion } \\
\text { crassflow is specified }\end{array}$ & $\begin{array}{l}\text { Fraction of axial flow } \\
\text { diverted thru gap } \mathrm{K} \\
\text { for grid type I }\end{array}$ \\
\hline SYMBOL & J & $\operatorname{CD}(\mathrm{J}, \mathrm{I})$ & K & $\operatorname{FXFL\emptyset W(K,I)}$ \\
\hline
\end{tabular}

Read if

$J 6=2,3$

Read NGRIDT

sets of

NCHANL cards 
CØBRA/CFTL INPUT

\begin{tabular}{|c|c|c|c|c|}
\hline WORD & 1 & 2 & 3 & 4 \\
\hline COLUMN: & $1-5$ & $6-10$ & $11-15$ & $16-20$ \\
\hline FORMAT & I 5 & I 5 & I 5 & I 5 \\
\hline $8 A$ & $\begin{array}{l}\text { Group number } \\
(=8)\end{array}$ & $\begin{array}{l}\text { Number of cards } \\
\text { of rod data }\end{array}$ & $\begin{array}{l}\text { Total number } \\
\text { of rods }\end{array}$ & $N C=\begin{array}{l}0 \text {, fuel properties not read } \\
>0 \text {, fuel projerties read }\end{array}$ \\
\hline SYMBOL & NGR@UP & N1 & $N 2=N R \emptyset D$ & $N 3=N C$ \\
\hline
\end{tabular}

Rod Data

\begin{tabular}{|c|c|c|}
\hline WORD & $\therefore$ & 6 \\
\hline COLUMN & $21-35$ & $36-50$ \\
\hline FORMAT & i0X, I 5 & $10 x, I 5$ \\
\hline $\begin{array}{c}8 \mathrm{~A} \\
\text { (Cọt.) }\end{array}$ & $\begin{array}{l}\text { Additional fuel model options } \\
=0 \text {, no additional options } \\
=4 \text {, circumferential rod conduction }\end{array}$ & $\begin{array}{l}\text { Logical unit from which rod input data to be read } \\
=0 \text {, read from cards } \\
>0 \text {, number of logical unit }\end{array}$ \\
\hline SYMBOL & $N 6=N O A X$ & $1 \$ 9^{\circ}$ \\
\hline
\end{tabular}

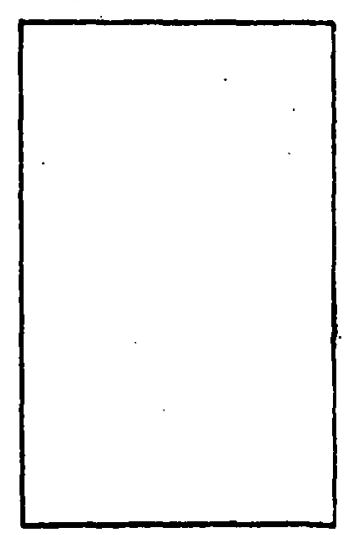

\begin{tabular}{|c|c|}
\hline WORD & 7 \\
\hline COLUMN & $51-55$ \\
\hline FORMAT & I 5 \\
\hline $\begin{array}{c}8 A \\
\text { (Cont.) }\end{array}$ & $\begin{array}{l}\text { Maximum number of subchannells connected to a rod } \\
\text { (Default }=6) \quad 7 \text { for CFTL with Hanger Channels }\end{array}$ \\
\hline SYMBOL & $N 10=M S C$ \\
\hline
\end{tabular}

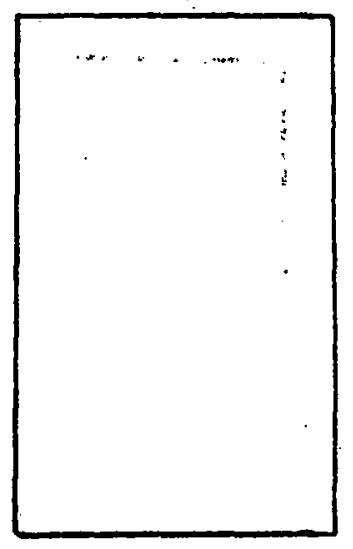


COBRA $/ C=T L$ INPUT

\begin{tabular}{|c|c|c|c|c|c|}
\hline WORD & 1 & 2 & 3 & \multicolumn{2}{|c|}{$4-15$} \\
\hline COLUMN & $1-5$ & $6-10$ & $11-15$ & \multicolumn{2}{|c|}{$16-75$} \\
\hline FORMAT & I 5 & $\mathrm{E} 5.2$ & $E 5.2$ & \multicolumn{2}{|c|}{$6(I 5, E 5.2)$} \\
\hline $8 B$ & Rod number & $\begin{array}{l}\text { Cladding outer } \\
\text { diameter (mm) }\end{array}$ & $\begin{array}{l}\text { Radial power } \\
\text { factor }\end{array}$ & $\begin{array}{l}\text { Subchannel number } \\
\text { adjacent to rod }\end{array}$ & $\begin{array}{l}\text { Fraction of total } \\
\text { power to subchannel }\end{array}$ \\
\hline SYMBOL & I & DR & RADIAL & \multicolumn{2}{|c|}{$(L R(I, L), \operatorname{PHI}(I, L), L=1,6)$} \\
\hline
\end{tabular}

Read $N 1$ sets of cards 8B and $8 \mathrm{BB}$

\begin{tabular}{|c|c|c|}
\hline WORD & & 1 \\
\hline COLUMN & & $16-25$ \\
\hline FORMAT & & $15 x, 15, E 5.2$ \\
\hline $8 B B$ & $\begin{array}{l}\text { Subchannel number } \\
\text { adjacent to rod }\end{array}$ & $\begin{array}{l}\text { Fraction of total } \\
\text { power to subchannel }\end{array}$ \\
\hline SYMBOL & \multicolumn{2}{|c|}{$\operatorname{LR}(I, L), \operatorname{PHI}(I ; L), L=7, \operatorname{MSC}$} \\
\hline
\end{tabular}

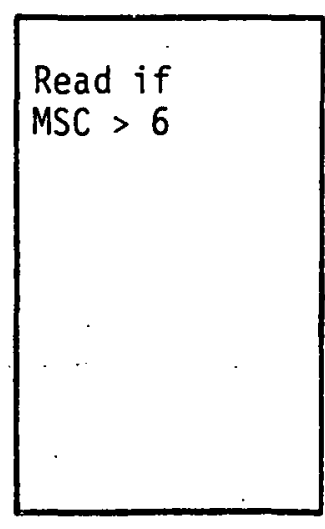

\begin{tabular}{|l|c|}
\hline WORD & $1-5$ \\
\hline COLUMN & $1-50$ \\
\hline FORMAT & $5 F 10.0$ \\
\hline $8 C 1$ & Fuer zonal radii \\
& \\
& \\
\hline SYMBOL & $R F(J), J=1,5$ \\
\hline
\end{tabular}

\section{Read if} $\mathrm{NC}>0$ 
COBRA/CFTL INPUT

\begin{tabular}{|l|c|}
\hline WORD & $1-5$ \\
\hline COLUMN & $1 .-50$ \\
\hline FORMAT & $5 F 10.0$ \\
\hline & Fuel conductivity \\
$8 C 2$ & \\
& \\
\hline & \\
\hline
\end{tabular}

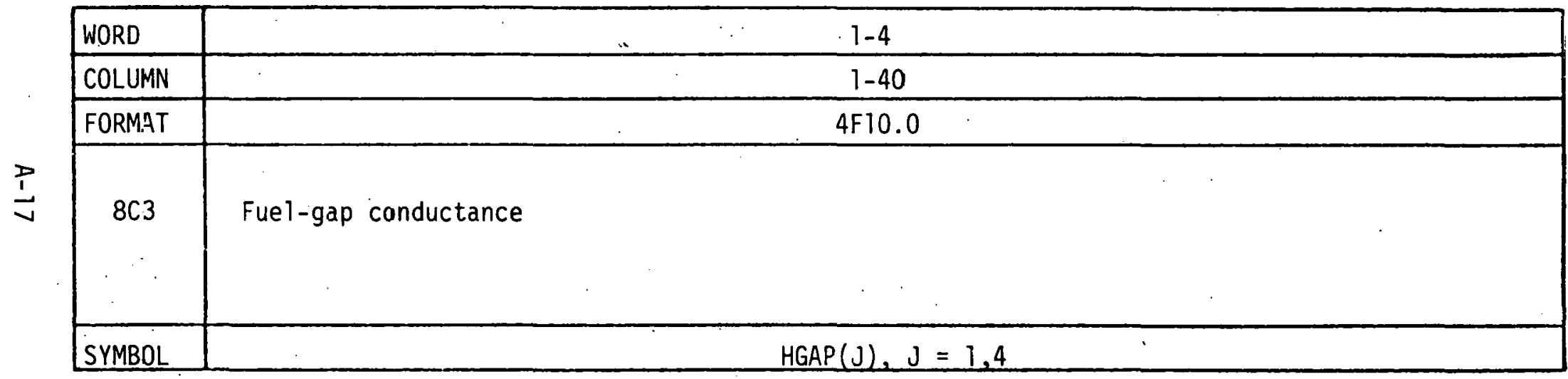

\begin{tabular}{|c|c|c|}
\hline WORD & & $1-5$ \\
\hline COLUMN & & $7-50$ \\
\hline FORMAT & 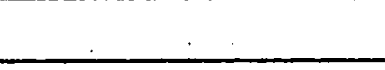 & $5 F 10.0$ \\
\hline $8 C 4$ & Fuel specific heat & 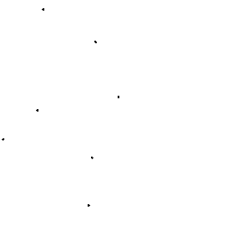 \\
\hline & & (J), $\mathrm{J}=1,5$ \\
\hline
\end{tabular}


COBRA,'CFTL INPUT

\begin{tabular}{|l|c|}
\hline WORD & $1-5$ \\
\hline COLUMN & $1-50$ \\
\hline FORMAT & $5 F 10.0$ \\
\hline $8 C 5$ & Fuel power fraction \\
& \\
& \\
\hline SYMBOL & \\
\hline
\end{tabular}

\begin{tabular}{|c|c|c|}
\hline WORD : & & $i-5$ \\
\hline COLUMN & & $i-50$ \\
\hline FORMAT & . & $5 F 10.0$ \\
\hline $8 \mathrm{C6}$ & Fuel density & \\
\hline SYMBOL & & $\operatorname{RHOF}(i), J=1,5$ \\
\hline
\end{tabular}

\begin{tabular}{|c|c|}
\hline WORD & 1 \\
\hline COLUMN & $1-5$ \\
\hline FORMAT & I 5 \\
\hline 8D1 & $\begin{array}{l}\text { Rods for calculating circumferential conductian } \\
=0 \text {, all rods } \\
>0 \text {, read NCONR rods for corduction (Card } 8 D 2 \text { ) }\end{array}$ \\
\hline SYMBOL & NCONR \\
\hline
\end{tabular}

Read if
$N 6=4$


COBRA/CFTL INPUT

\begin{tabular}{|l|c|}
\hline WORD & $1-11$ \\
\hline COLUMN & $1-55$ \\
\hline FORM:AT & $11(15)$ \\
\hline $8 D 2$ & Rod numbers for circumferential conduction \\
& \\
\hline SYMBOL & ICONR(I), I $=1$, NCONR \\
\hline
\end{tabular}

Read if

NCONR $>0$

Read as many

cards as

necessary.

Calculational

Variables

\begin{tabular}{|l|c|}
\hline WORD & 4 \\
\hline COLUMN & $16-40$ \\
\hline FORMAT & $20 \times$, I5 \\
\hline $\begin{array}{c}9 A \\
\text { (Cont.) }\end{array}$ & $=0$, let code calculate equal axial node locations \\
& $=i$, read axial node locations (Card 9CC) \\
\hline SYMBOL & N7 \\
\hline
\end{tabular}

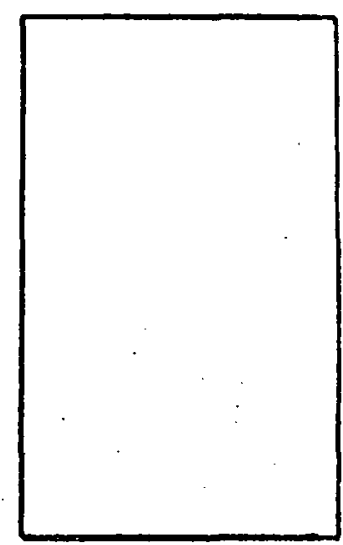


CØBRA, CFTL INPUT

\begin{tabular}{|l|c|c|c|c|c|}
\hline WORD & 1 & 2 & 3 & 4 & 5 \\
\hline COLUMN & $1-5$ & $6-10$ & $11-15$ & $16-20$ & $21-25$ \\
\hline FORMAT & E5.0 & E5.0 & E5.0 & E5.0 & E5.0 \\
\hline 9B & $\begin{array}{l}\text { Total axial } \\
\text { length (mm) }\end{array}$ & $\begin{array}{l}\text { Total transient } \\
\text { time (sec.) }\end{array}$ & $\begin{array}{l}\text { External crossflow } \\
\text { convergence factor } \\
\text { (Default }=0.1)\end{array}$ & $\begin{array}{l}\text { Internal crossflow } \\
\text { convergence factor } \\
\text { (Default }=0.001) .\end{array}$ & $\begin{array}{l}\text { External axial } \\
\text { flow convergence } \\
\text { factor } \\
\text { (Default }=0.01)\end{array}$ \\
\hline SYMBOL & $Z$ & TTIME & WERRX & WERRY & FERRQR \\
\hline
\end{tabular}

\begin{tabular}{|c|c|c|c|c|c|}
\hline WORD & 6 & 7 & 8 & 9 & 10 \\
\hline COLUMN & $26-30$ & $31-35$ & $36-40$ & $41-45$ & $46-50$ \\
\hline FORMAT & E5.0 & $E 5.0$ & E5.0 & E5.0 & E5.0 \\
\hline $\begin{array}{c}9 \mathrm{~B} \\
\text { (Cont.) }\end{array}$ & $\begin{array}{l}\text { Turbulent crossflow } \\
\text { resistance factor } \\
(\text { Default }=0.5)\end{array}$ & $\begin{array}{l}\text { Transverse } \\
\text { momentum } \\
\text { factor } \\
\text { (Default=0.5) }\end{array}$ & $\begin{array}{l}\text { Turbulent } \\
\text { momentum } \\
\text { factor } \\
\text { (Default = } \\
0.0 \text { ) }\end{array}$ & $\begin{array}{l}\text { Bundle } \\
\text { orientation } \\
\text { from } \\
\text { vertical } \\
\text { [0=up](deg.) }\end{array}$ & $\begin{array}{l}\text { Contribution of velocity from } \\
\text { donor and receiver subchannels } \\
\text { in } U^{*} \text { calculations } \\
0 \leq U S D Q N \leq 1 \\
(D e f a u T t=0.0) \\
\end{array}$ \\
\hline SYMBOL & $\mathrm{KIJ}$ & $S L$ & FTM & THETA & USDØN \\
\hline
\end{tabular}

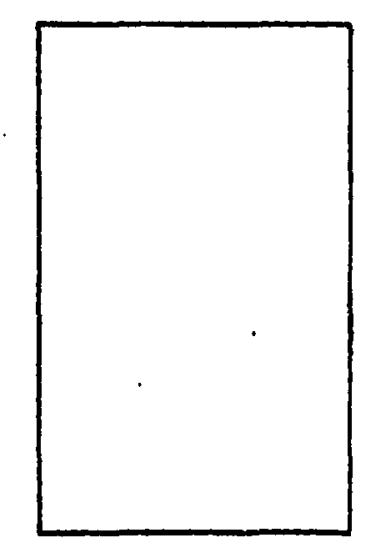

\begin{tabular}{|l|c|c|c|}
\hline WORD & 11 & 12 & 13 \\
\hline COLUMN & $51-55$ & $56-60$ & $61-65$ \\
\hline FORMAT & $E 5.0$ & $E 5.0$ & E5.0 \\
\hline $\begin{array}{c}9 B \\
\text { (Cont.) }\end{array}$ & $\begin{array}{l}\text { Damping factor for iterative } \\
\text { SP term }\end{array}$ & $\begin{array}{c}\text { Crossflow solution accelerator } \\
\text { Damping factor for } \\
\text { iterative axial flow }\end{array}$ \\
\hline SYMBOL & DAMPNG & (Default = 1.5) & (Default $=1.0)$ \\
\hline
\end{tabular}

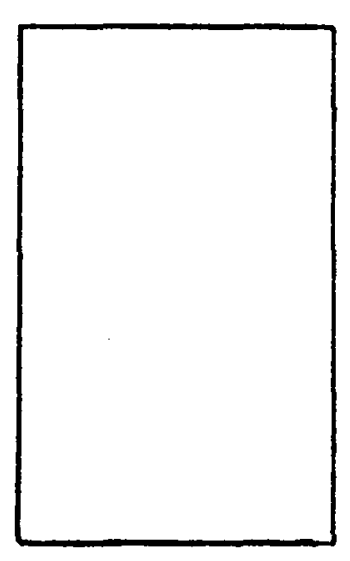




\begin{tabular}{|c|c|c|c|c|c|}
\hline WORD & 1 & 2 & 3 & 4 & 5 \\
\hline COLUMN & $1-5$ & $6-10$ & $11-15$ & $16-20$ & $21-25$ \\
\hline FORMAT & I 5 & I 5 & I 5 & I 5 & I 5 \\
\hline $9 C$ & $\begin{array}{l}\text { Number of } \\
\text { axial } \\
\text { nodes }\end{array}$ & $\begin{array}{l}\text { Total number } \\
\text { of time steps } \\
\text { allowed } \\
\text { DT }=\frac{\text { TTIME }}{\text { NDT }}\end{array}$ & $\begin{array}{l}\text { Max. number of } \\
\text { external itera- } \\
\text { tions allowed } \\
\text { (implicit scheme) } \\
\text { (Default }=20)\end{array}$ & $\begin{array}{l}\text { Max. number of } \\
\text { internal itera- } \\
\text { tions allowed } \\
\text { (implicit scheme) } \\
\text { (Default }=\text { max. of } \\
20 \text { or } 2 \star \text { no. gaps) }\end{array}$ & $\begin{array}{l}\text { Min number of iterations } \\
\text { in internal crossflow } \\
\text { solution } \\
\text { (implicit scheme) } \\
\text { (Default }=5 \text { ) }\end{array}$ \\
\hline SYMBOL & NDX & NDT & NTRIES & ITRY & ITRYM \\
\hline
\end{tabular}

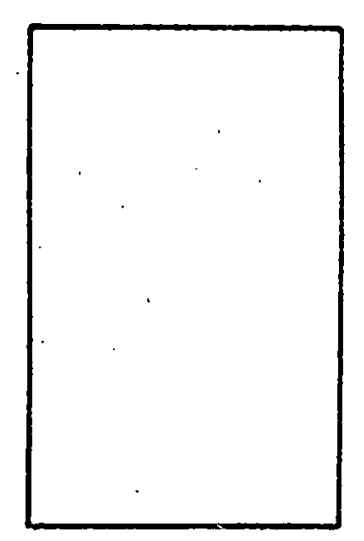

\begin{tabular}{|l|c|c|}
\hline WORD & 6 & 7 \\
\hline COLUMN & $26-30$ & $31-35$ \\
\hline FORMAT & E5.0 & E5.0 \\
\hline$\stackrel{1}{\sim}$ & $\begin{array}{l}\text { Distance from inlet where } \\
\text { roughening begins (mm) }\end{array}$ & $\begin{array}{l}\text { Distance from inlet where } \\
\text { roughening ends (mm) }\end{array}$ \\
\hline (Cont.) & & \\
\hline SYMBOL & LENSMQ & LENRQU \\
\hline
\end{tabular}

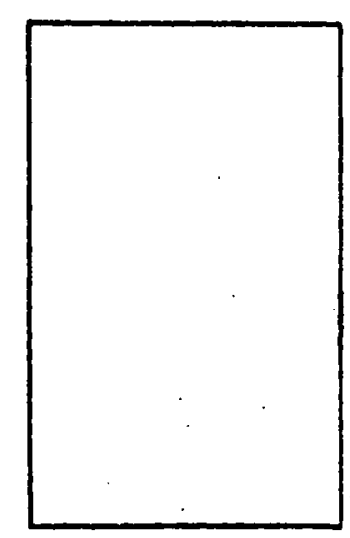

\begin{tabular}{|c|c|c|}
\hline WORD & & $1-6$ \\
\hline COLUMN & & $1-60$ \\
\hline FORMAT & & $6 E 10.5$ \\
\hline $9 C C$ & $\begin{array}{l}\text { Axial node locations } \\
\text { beginning with zero } \\
(\mathrm{mm})\end{array}$ & $\begin{array}{l}\text { Do not include a node at the bundle exit. } \\
\text { This is automatically done. }\end{array}$ \\
\hline SYMBOL & $X(J), J=1, N D X$ & \\
\hline
\end{tabular}


CQBRA/CFTL INPUT

\begin{tabular}{|c|c|c|c|}
\hline WORD & 1 & \multicolumn{2}{|c|}{2} \\
\hline COLUMN & $1-5$ & \multicolumn{2}{|c|}{$6-10$} \\
\hline FORMAT & I 5 & \multicolumn{2}{|c|}{15} \\
\hline $10 A$ & $\begin{array}{l}\text { Group number } \\
(=10)\end{array}$ & $\begin{array}{l}\text { Single phase mixing option } \\
=0, W^{\prime}=A B E T A^{\star}\left(S_{K} \bar{G}\right) \\
=1, w^{\prime}=A B E T A^{\star} R^{\star \star} B B E T A^{\star}\left(S_{K} \bar{G}\right) \\
=2, W^{\prime}=A B E T A * R e^{\star \star B B E T A *}(\overline{D G})\end{array}$ & 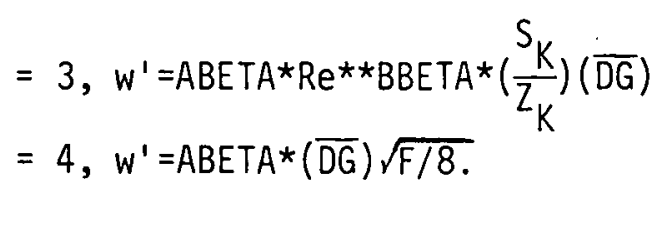 \\
\hline SYMBOL & NGRØUP & & $\mathrm{NSCBC}$ \\
\hline
\end{tabular}

Turbulent

Mixing

Correlation

\begin{tabular}{|c|c|}
\hline WORD & 3 \\
\hline COLUMN & $11-20$ \\
\hline FORMAT & $5 x, I 5$ \\
\hline $\begin{array}{l}10 \mathrm{~A} \\
\text { (Cont.) }\end{array}$ & $\begin{array}{l}\text { Radial thermal conduction mixing } \\
=0 \text {, no thermal conduction } \\
=1 \text {, read thermal conduction factor GK (Card 1OC) }\end{array}$ \\
\hline SYMBOL & $\mathrm{N} 3=\mathrm{J} 5$ \\
\hline
\end{tabular}

\begin{tabular}{|l|c|c|}
\hline WORD & 1 & 2 \\
\hline COLUMN & $1-5$ & $6-10$ \\
\hline FORMAT & F5.3 & F5.3 \\
\hline 1OB & $\begin{array}{l}\text { Turbulent mixing correlation constant } \\
(\text { see Card } 10 \mathrm{~A})\end{array}$ & $\begin{array}{l}\text { Turbulent mixing correlation constant } \\
(\text { See Card 1OA) } \\
\text { (omit if NSCBC }=0 \text { or 4) }\end{array}$ \\
\hline SYMBOL & ABETA & BBETA \\
\hline
\end{tabular}

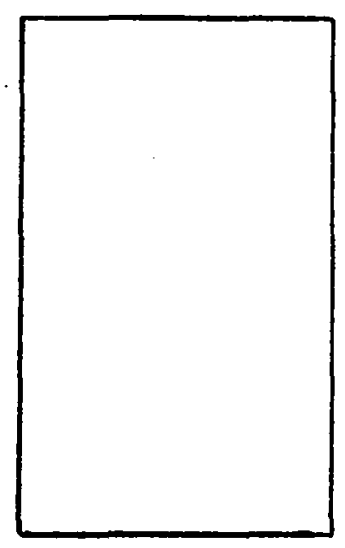




\begin{tabular}{|l|c|}
\hline WORD & 1 \\
\hline COLUUMN & $1-5$ \\
\hline FORMAT & F5.3 \\
\hline IOC & Geometry factor for radial thermal conduction mixing \\
& 1 \\
\hline SYMBOL & GK \\
\hline
\end{tabular}

\begin{tabular}{|c|c|c|c|}
\hline WORD & 1 & 2 & 3 \\
\hline COLUMN & $1-5$ & $6-10$ & $11-15$ \\
\hline FORMAT & I 5 & I 5 & I 5 \\
\hline $17 \mathrm{~A}$ & $\begin{array}{l}\text { Group number } \\
(=11)\end{array}$ & $\begin{array}{l}\text { Inlet enthalpy or temp. option } \\
=0 \text {, inlet } h=H I N \\
=1 \text {, inlet } T=H I N \\
=2 \text {, read inlet } h \text { for each subchannel } \\
=3 \text {, read inlet } T \text { for each subchannel }\end{array}$ & $\begin{array}{l}\text { Inlet mass flux option } \\
=0 \text {, inlet } G=G I N \text { for each subchannel } \\
=1 \text {, equal } \Delta P / \Delta X \text { across first axial node } \\
=2 \text {, flow split by flow fractions given } \\
\quad \text { in Card } 11 D\end{array}$ \\
\hline SYMBOL & NGRØUP & $\mathrm{NT}=\mathrm{IH}$ & $N 2=I G$ \\
\hline
\end{tabular}

\begin{tabular}{|c|c|c|c|c|}
\hline WORD & 4 & 5 & 6 & 7 \\
\hline COLUMN & $16-20$ & $27-25$ & $26-30$ & $31-35$ \\
\hline FORMAT & I 5 & I 5 & I 5 & 15 \\
\hline $\begin{array}{l}11 \mathrm{~A} \\
\text { (Cont.) }\end{array}$ & $\begin{array}{l}\text { Transient forcing } \\
\text { function for } \\
\text { system pressure } \\
\text { (Card } 11 E \text { ) }\end{array}$ & $\begin{array}{l}\text { Transient forcing } \\
\text { function for inlet } \\
h \text { or } T \\
\text { (Card } 11 F \text { ). }\end{array}$ & $\begin{array}{l}\text { Transient forcing } \\
\text { function for inlet } \\
G \text { or } \Delta P \\
\text { (Card } 17 G \text { ) }\end{array}$ & $\begin{array}{l}\text { Transient forcing } \\
\text { function for average } \\
\text { heat flux } \\
\text { (Card } 11 \mathrm{H} \text { ) }\end{array}$ \\
\hline SYMBOL & $N 3=N P$ & $\mathrm{~N} 4=\mathrm{NH}$ & $N 5=N G$ & $N 6=N Q$ \\
\hline
\end{tabular}

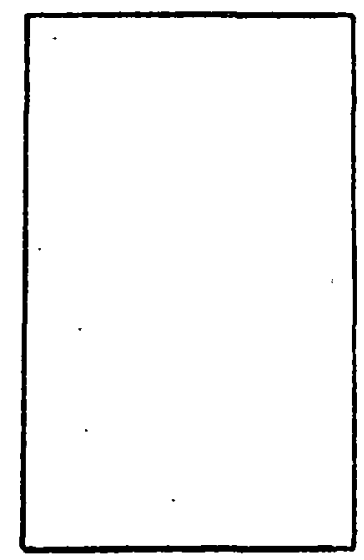


CØBRA/CFTL INPUT

\begin{tabular}{|l|c|c|c|c|}
\hline WORD & 1 & 2 & 3 & 4 \\
\hline COLUMN & $1-10$ & $11-20$ & $21-30$ & $31-40$ \\
\hline FORMAT & $\mathrm{F} 10.0$ & $\mathrm{~F} 10.0$ & $\mathrm{Fl0.0}$ & $\mathrm{Fl0} .0$ \\
\hline IIB & System Pressure & Inlet $\mathrm{h}$ or T depending on $\mathrm{HH}$ & Inlet mass flux & Average heat flux \\
& $(\mathrm{KPa})$ & $\left(\mathrm{KJ} / \mathrm{Kg}\right.$ or $\left.{ }^{\circ} \mathrm{C}\right)$ & $\left(\frac{\mathrm{g}}{\mathrm{s}-\mathrm{mm}^{2}}\right)$ & $\left(\frac{\mathrm{KW}}{\mathrm{m}^{2}}\right)$ \\
\hline SYMBOL & PEXIT & $\mathrm{HIN}$ & $\mathrm{GIN}$ & AFLUX \\
\hline
\end{tabular}

\begin{tabular}{|c|c|}
\hline WORD & $1-12$ \\
\hline COLUMN & $1-50$ \\
\hline FORMAT & $12 E j .0$ \\
\hline 110 & $\begin{array}{l}\text { Inlet enthalpy }(\mathrm{IH}=2) \text { or inlet temperature }(\mathrm{IH}=3) \text { for each subchancel. } \\
\left(\begin{array}{l}\mathrm{KJ} \\
\mathrm{Kg}\end{array}{ }^{\circ} \mathrm{C}\right)\end{array}$ \\
\hline SYMBOL & $\operatorname{HINLET}(\mathrm{I} I, I=1$, NCHANL \\
\hline
\end{tabular}

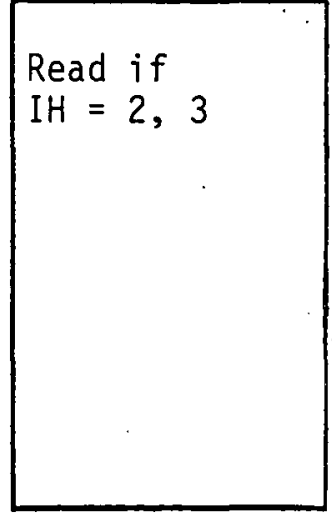

\begin{tabular}{|l|c|}
\hline WORD & $1-2$ \\
\hline COLUMN & $1-60$ \\
\hline FORMAT & $\begin{array}{c}\text { Individual subchannel inlet flow fraction } \\
\text { F(I)/FTOTAL }\end{array}$ \\
\hline 110 & FINLET(I:, I $=1$, NCHANL \\
\hline
\end{tabular}

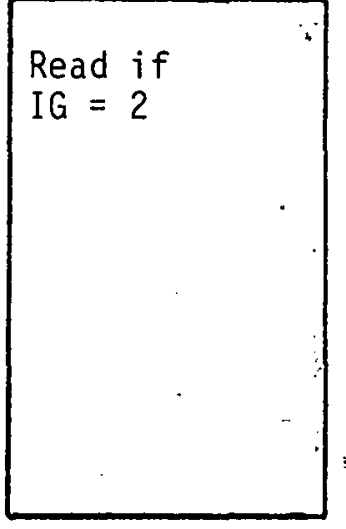


CØBRA/CFTL INPUT

\begin{tabular}{|l|c|c|}
\hline WORD & \multicolumn{2}{|c|}{$1-12$} \\
\hline COLUMN & \multicolumn{2}{|c|}{$1-60$} \\
\hline FORMAT & \multicolumn{2}{|c|}{$12 \mathrm{E} 5.0$} \\
\hline $11 E$ & \multicolumn{2}{|c|}{ Pressure Transient Table } \\
\cline { 2 - 3 } & $\begin{array}{l}\text { Transient time when } \\
\text { factor is applied }\end{array}$ & Fraction of SS \\
system pressure
\end{tabular}

\begin{tabular}{|c|c|c|}
\hline WORD & $\cdot$ & $1-12$ \\
\hline COLUNN & & $1-60$ \\
\hline FORMAT & & $12 E 5.0$ \\
\hline \multirow{2}{*}{$11 \mathrm{~F}$} & \multicolumn{2}{|c|}{ Enthalpy or Temperature Transient Table } \\
\hline & $\begin{array}{l}\text { Transient time } \\
\text { when factor } \\
\text { applied. }\end{array}$ & $\begin{array}{l}\text { Fraction of inlet enthalpy } \\
\text { (IH }=0 \text { or } 2) \text { or inlet } \\
\text { temperature }(I H=1 \text { or } 3)\end{array}$ \\
\hline SYMBOL & YH $H(I)$ & $\mathrm{FH}(\mathrm{I}), \mathrm{I}=1, \mathrm{NH}$ \\
\hline
\end{tabular}

\begin{tabular}{|c|c|c|}
\hline WORD & \multicolumn{2}{|c|}{$1-12$} \\
\hline COLUMN & \multicolumn{2}{|c|}{$1-60$} \\
\hline FORMAT & \multicolumn{2}{|c|}{$12 \mathrm{E} 5.0$} \\
\hline \multirow{2}{*}{$11 G$} & \multicolumn{2}{|c|}{ Inlet Flow Boundary Condition Transient Table } \\
\hline & $\begin{array}{l}\text { Transient time } \\
\text { when factor is } \\
\text { applied }\end{array}$ & $\begin{array}{l}\text { Fraction of SS } \\
\text { inlet flow }\end{array}$ \\
\hline SYMBOL & $Y G(I)$. & $F G(I), I=1, N G$ \\
\hline
\end{tabular}


CØBRA;CFTL INPUT

\begin{tabular}{|l|c|c|}
\hline WORD & \multicolumn{2}{|c|}{$1-12$} \\
\hline COLUMN & \multicolumn{2}{|c|}{$1-60$} \\
\hline FORMAT & \multicolumn{2}{|c|}{2 E5 .0 } \\
\hline $11 \mathrm{H}$ & \multicolumn{1}{|c|}{ Heat Flux Transient Table } \\
\hline SYMBOL & $\begin{array}{l}\text { Transient time } \\
\text { when factor is } \\
\text { applied }\end{array}$ & $\begin{array}{l}\text { Fraction of SS } \\
\text { heat flux }\end{array}$ \\
\hline
\end{tabular}

\begin{tabular}{|c|c|c|}
\hline WORD & 1 & 2 \\
\hline COLUMN & $1-5$ & $6-10$ \\
\hline FORMAT & I 5 & 15 \\
\hline $12 A$ & $\begin{array}{l}\text { Group number } \\
(=12)\end{array}$ & $\begin{array}{l}=10 \text {, subchannel data only } \\
=11 \text {, subchannel data and crossflows } \\
=12 \text {, subchannel data, fuel rod and duct temperatures } \\
=13 \text {, subchannel data, crosiflows, fuel rod, and duct temperatures }\end{array}$ \\
\hline SYMBOL & NGRQUP & $N 1=N \emptyset U T$ \\
\hline
\end{tabular}

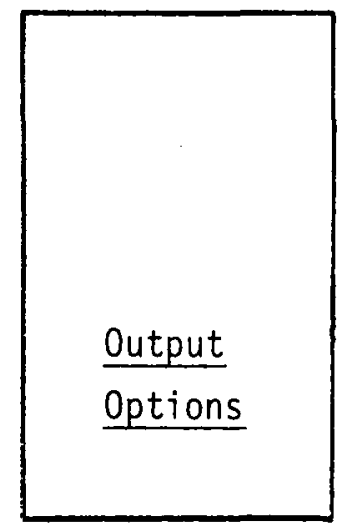

\begin{tabular}{|c|c|c|c|}
\hline WORD & 3 & 4 & 5 \\
\hline COLUMN & $11-15$ & $16-20$ & $20-30$ \\
\hline FORMAT & I 5 & I 5 & $5 X$, I 5 \\
\hline $\begin{array}{c}12 A \\
\text { (Cont.) }\end{array}$ & $\begin{array}{l}=0 \text {, print all subchannel data } \\
>0 \text {, read NPCHAN subchannel } \\
\text { numbers for printing } \\
\text { (Card 12B) }\end{array}$ & $\begin{array}{l}=0 \text {, print tata for a } 11 \text { rods } \\
>0 \text {, read } N \text { OROD rod numbers } \\
\text { for printing } \\
\text { (Card } 12 C)\end{array}$ & $\begin{array}{l}=0, \text { print crossflows for } \\
\text { all gaps } \\
>0, \text { read NPGAP gap numbers } \\
\text { for printing (Card 12D) }\end{array}$ \\
\hline SYMBOL & $N 2=N P C H A N$ & $N 3=N ? R \emptyset D$ & $N 5=: U P G A P$ \\
\hline
\end{tabular}

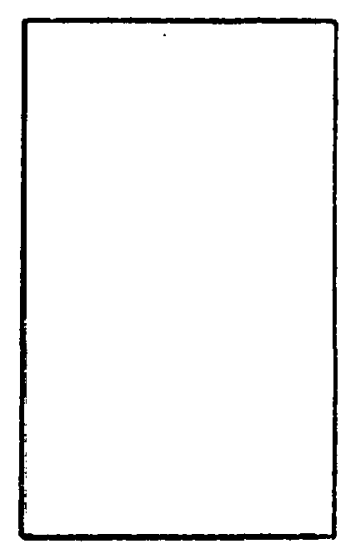


CØBRA/CFTL INPUT

\begin{tabular}{|l|c|}
\hline WORD & $1-24$ \\
\hline COLUMN & $1-72$ \\
\hline FORMPT & 2413 \\
\hline $12 B$ & Numbers of NPCHAN subchannels to be printed \\
& \\
& PRINTC (I),$I=1$, NPCHAN \\
\hline
\end{tabular}

\begin{tabular}{|c|c|c|}
\hline WORD & \multicolumn{2}{|c|}{$1-24$} \\
\hline COLUMN & \multicolumn{2}{|c|}{$1-72$} \\
\hline FORMAT & \multicolumn{2}{|c|}{2413} \\
\hline $12 C$ & $\begin{array}{l}\text { Indices of NPRQD rods to be printed } \\
\text { (heat flux and temperature) }\end{array}$ & Note: If use RODCON, must print for rod $\Perp$ 1. \\
\hline SYMBOL & PRINTR & $I=N P R \emptyset D$ \\
\hline
\end{tabular}

\section{Read if}

\begin{tabular}{|l|c|}
\hline WORD & $1-24$ \\
\hline COLUMN & $1-72$ \\
\hline FORMAT & 2413 \\
\hline 120 & $\begin{array}{l}\text { Number of NPGAP gaps to be printed } \\
\text { (crossflow) }\end{array}$ \\
\hline SYMBO: & PRINTG (I), I = I, NPGAP \\
\hline
\end{tabular}


СØBRA,'CFTL INPUT

\begin{tabular}{|c|c|}
\hline WORD & 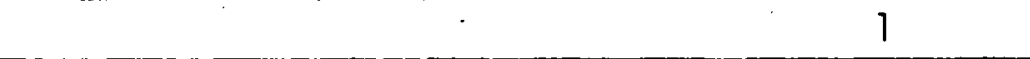 \\
\hline COLUMN. & $1-5$ \\
\hline FORMAT & [ 5 \\
\hline $13 A$ & $\begin{array}{l}\text { Group number } \\
(=1.3)\end{array}$ \\
\hline SYMBOL & NGRQUP \\
\hline
\end{tabular}

\begin{tabular}{|l|c|}
\hline WORD & $1-6$ \\
\hline COLUMN & $i-60$ \\
\hline FORMAT & $6 \mathrm{Fl0.0}$ \\
\hline 138 & $\begin{array}{l}\text { Tube radius for subchannel types I }\left(\begin{array}{l}\text { equivalent tube radi for wall and corner channels. } \\
\text { Should be zero for central and hanger channels. }\end{array}\right. \\
\text { SYMBOL }\end{array}$ \\
\hline
\end{tabular}

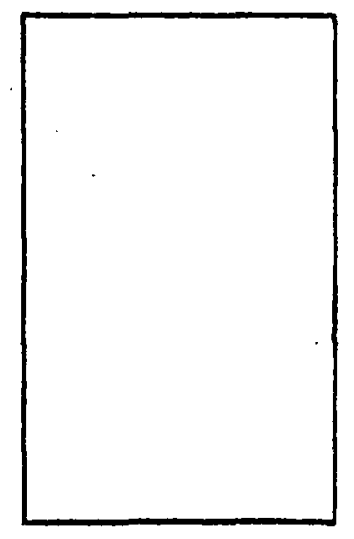

\begin{tabular}{|c|c|}
\hline WORD & $1-6$ \\
\hline COLUMN & $1-60$ \\
\hline FORMAT & $6 F 10.0$ \\
\hline $13 C$ & $\begin{array}{l}\text { Radius of zero shear for subchannel Types I. Must be read in as zero for } \\
\text { (malT and corner channels. }\end{array}$ \\
\hline SYMBOL & $R Z S(I), I=1, N F T$ \\
\hline
\end{tabular}

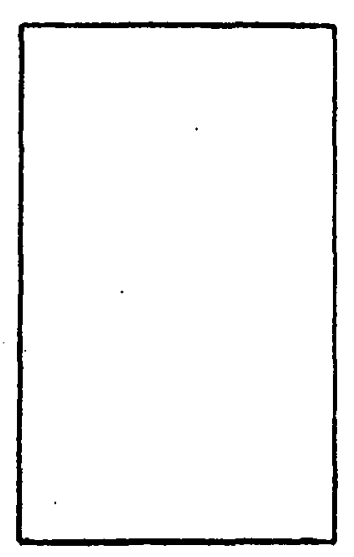


COBRA/CFTL INPUT

\begin{tabular}{|c|c|}
\hline WORD - & 1 \\
\hline COLUMN & $1-60$ \\
\hline FORMAT & $6 \mathrm{~F} 10.0$ \\
\hline 130 & Rod radius for subchannel types I (mm) \\
\hline SYMBOL & $\mathrm{RR}(\mathrm{I}), \mathrm{I}=1, \mathrm{NFT}$ \\
\hline
\end{tabular}

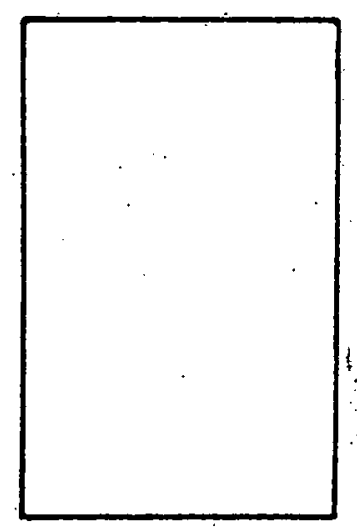

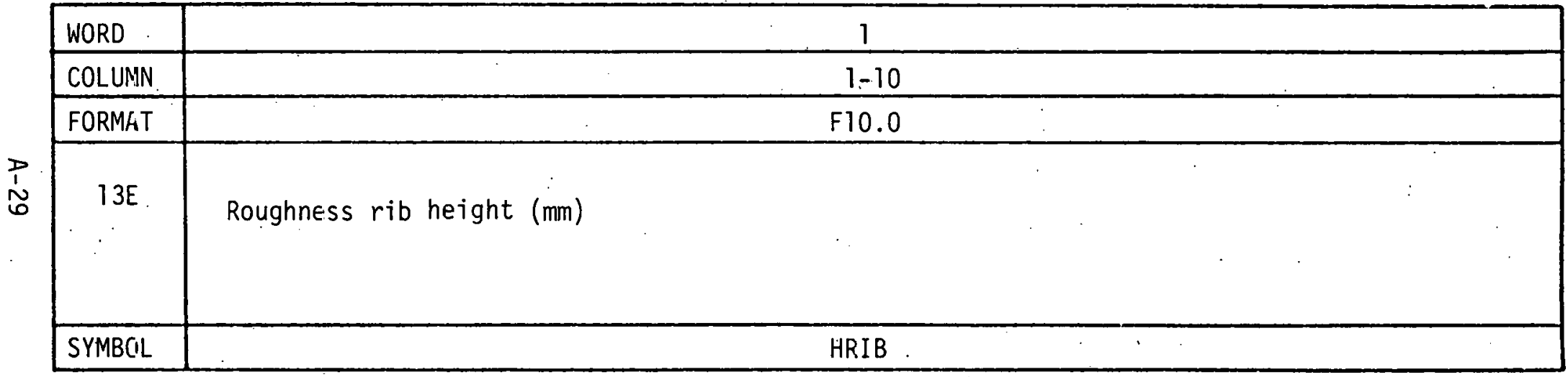

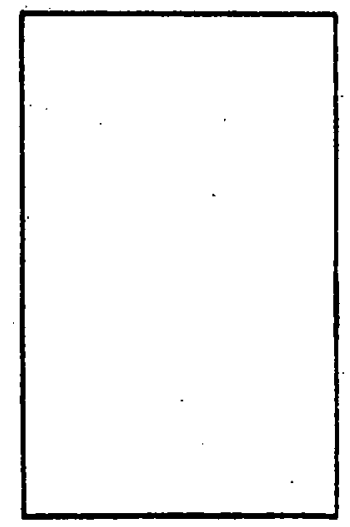

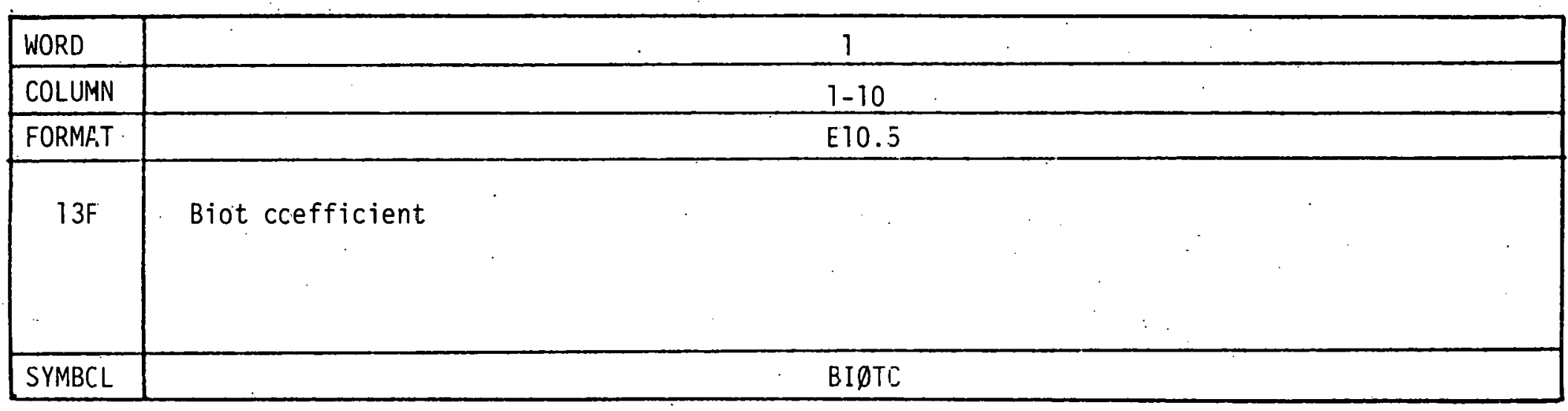

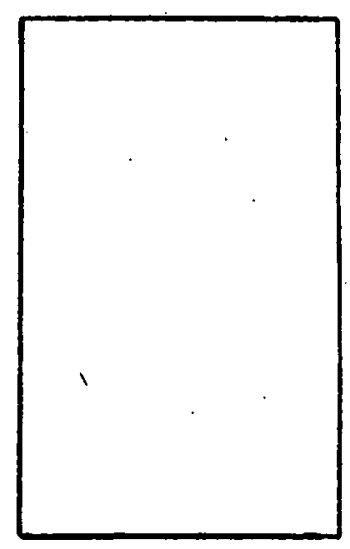




\begin{tabular}{|l|c|}
\hline WORD & $1-3$ \\
\hline COLUMN & $1-30$ \\
\hline FORMAT & $3 E 10.5$ \\
\hline $13 G$ & R-function coefficients. $\begin{array}{l}\text { Fully rough flow: }\left(H^{+}>C R(3)\right), R\left(H^{+}\right)=C R(1), A=C R(2) \\
\text { Transition region: } R\left(H^{+}\right)=C R(4)-C R(5) \ln \left(H^{+}\right) \\
\end{array}$ \\
\hline SYMBOL & $A=C R(6)-C R(7) \ln \left(H^{+}\right)+C R(8)\left[\ln \left(H^{+}\right)\right]^{2}$ \\
\hline
\end{tabular}

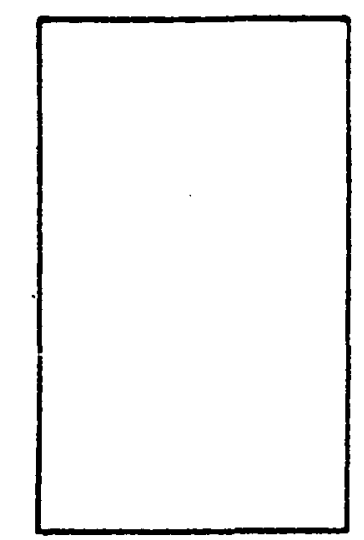

\begin{tabular}{|c|c|}
\hline WORD & $1-2$ \\
\hline COLUMN & $1-80$ \\
\hline FORMAT & $3 \mathrm{E} 10.5$ \\
\hline $13 \mathrm{H}$ & $\begin{array}{ll}\text { G-function coefficients. } & G\left(H W^{+}\right)=C G(1)\left[H N^{+}\right]^{C G(2)} *[P R]^{C G(4)} *[T W T B]^{C G(5)}, A H=C G(3) \\
& \text { If } H+<C G(6), j\left(H W^{+}\right)=C G(7), C G(8)=0 \text {, a spare for now. }\end{array}$ \\
\hline SYMBOL & $C G(I), I=1,8$ \\
\hline
\end{tabular}

\begin{tabular}{|l|c|}
\hline WORD & \\
\hline COLUMN & \\
\hline FORMAT & \\
\hline & indicates end of tata, begin execution \\
& \\
\hline SYMBOL & B $A$ N K \\
\hline
\end{tabular}

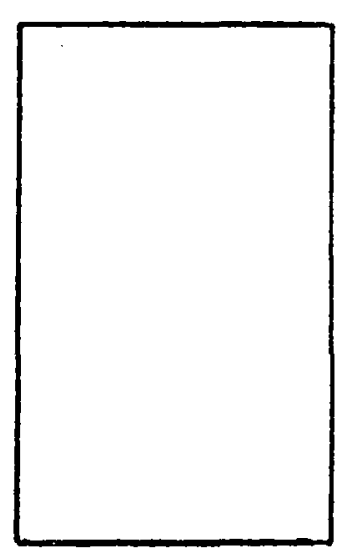


CØBRA/CFTL INPUT
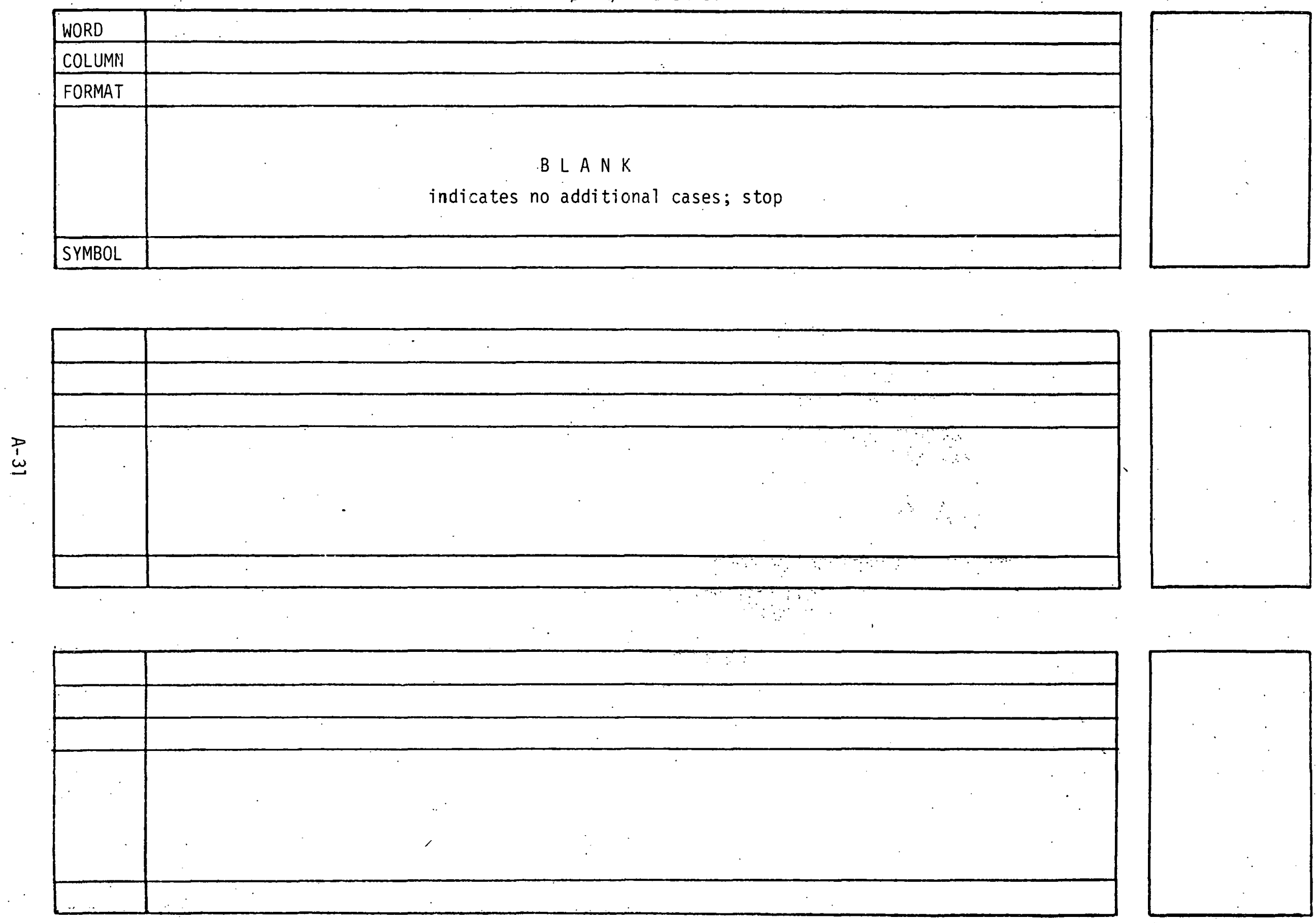


\section{THIS PAGE \\ WAS INTENTIONALLY LEFT BLANK}




\begin{abstract}
APPENDIX B
SAMPLE INPUT FOR COBRA/CFTL CODE
\end{abstract}

B-1 
DELT',L COBRA \#CFTL-PLOT.FILEZ, TPF \&.DATA

ELT BR1 $57401 C \quad 05 / 27 / 81 \quad 11: 21: 25 \quad 10->01$

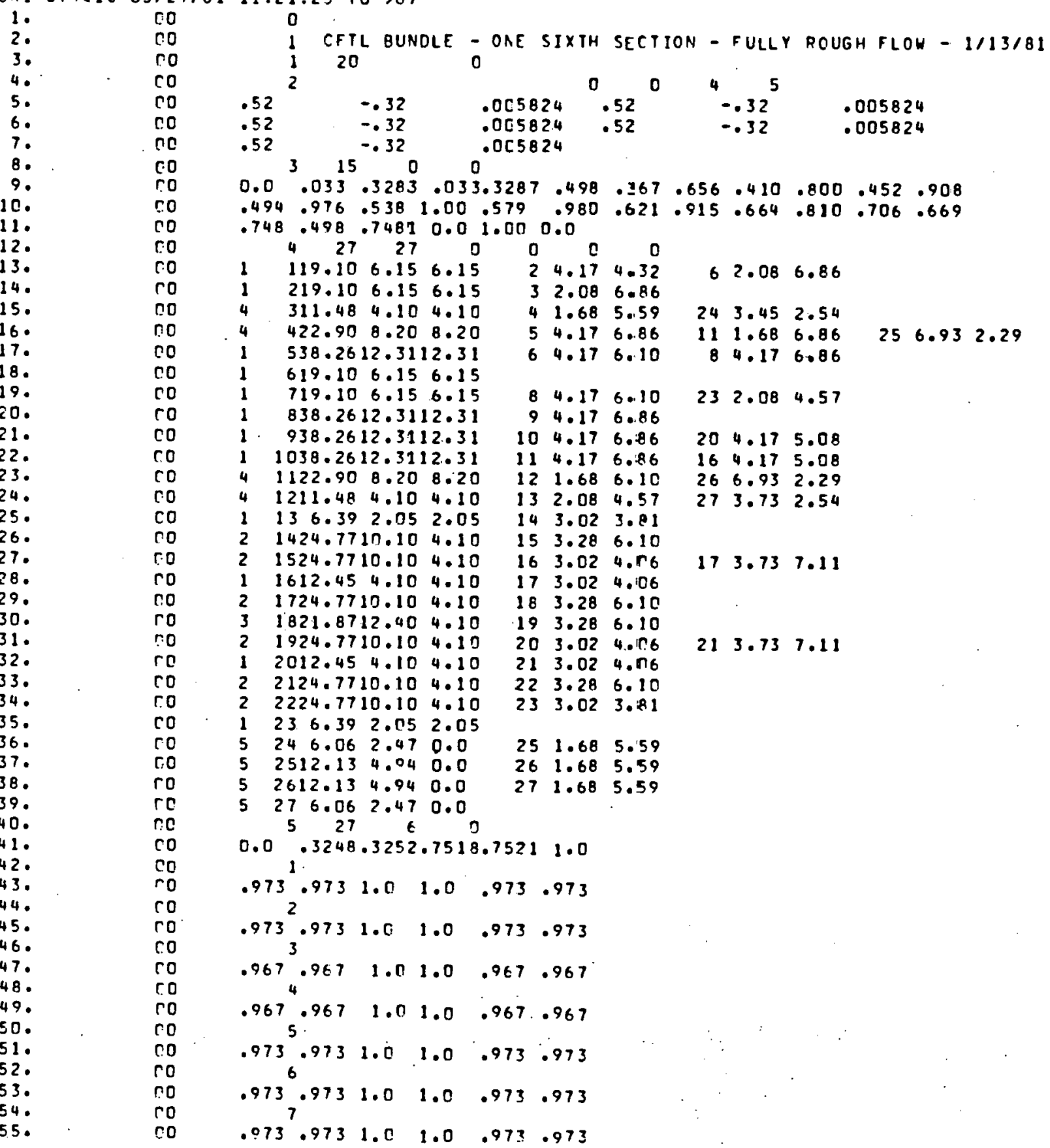




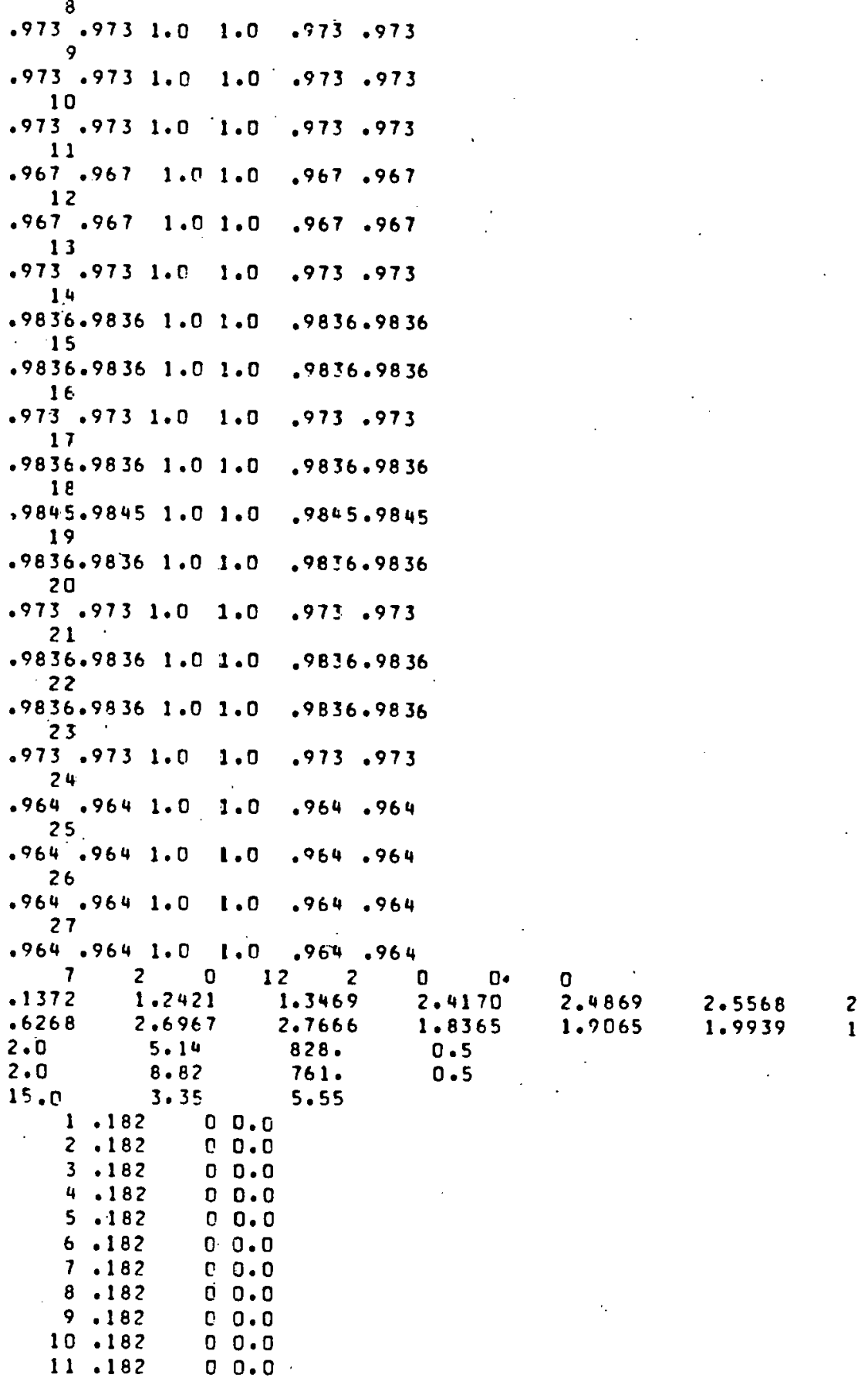




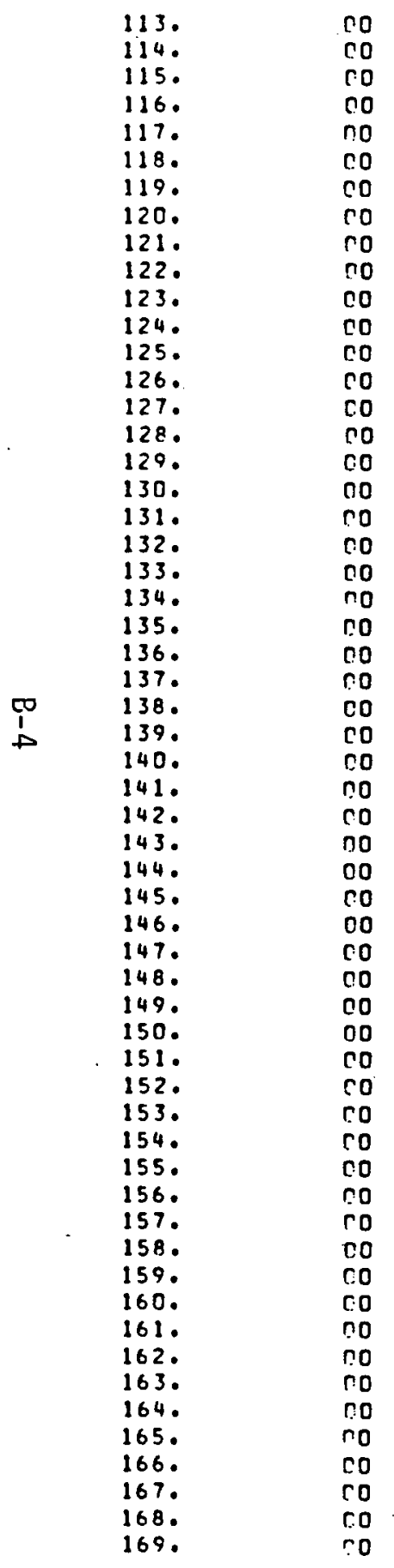

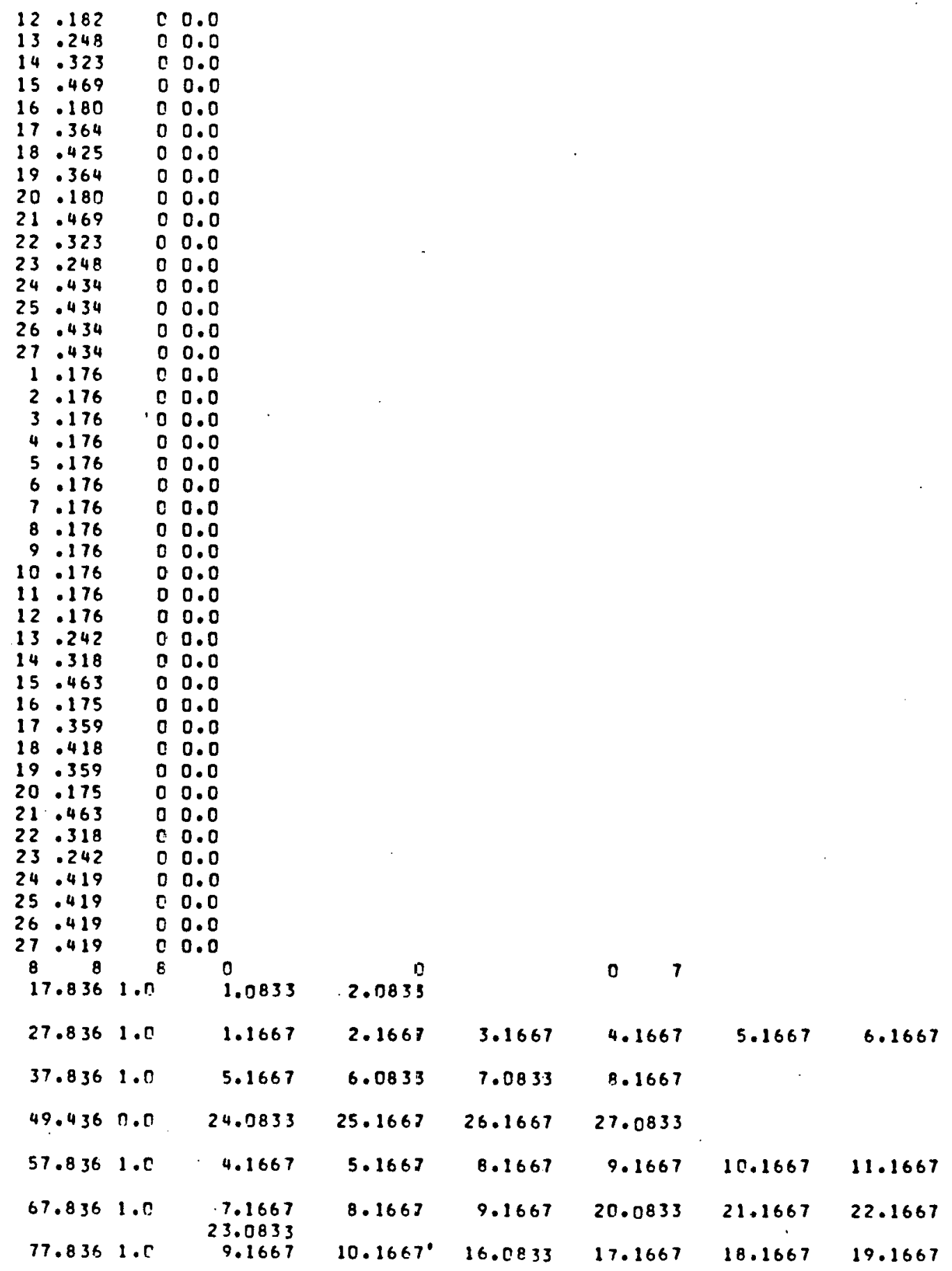




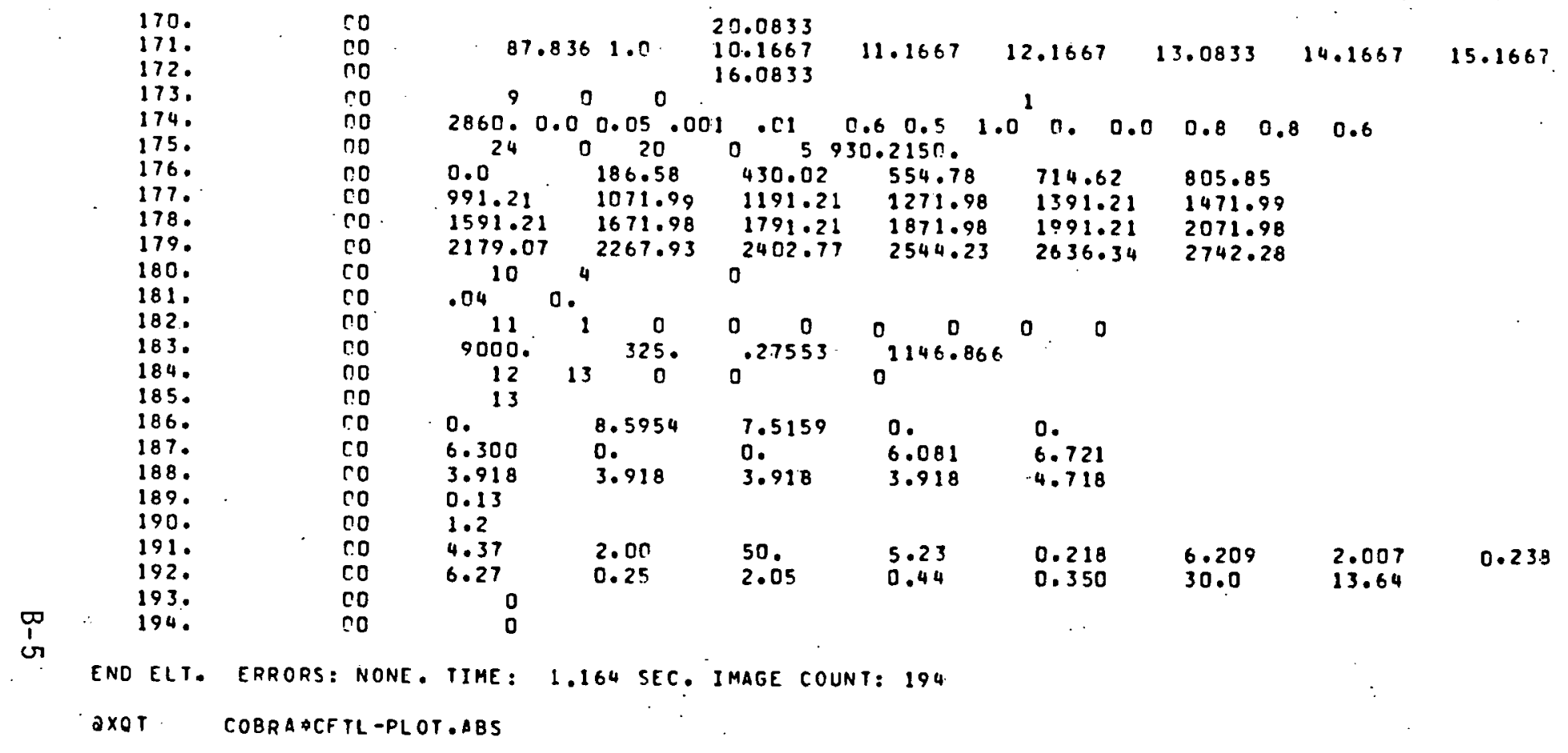




\section{THIS PAGE \\ WAS INTENTIONALLY \\ LEFT BLANK}




\section{APPENDIX C}

SAMPLE OUTPUT FOR COBRA/CFTL CODE 
THIS IS THE OAK RIDGE NATIONAL LABORATORY VERSION OF THE

COBRA-IV THERMAL/HYORAULIC ANALYSIS COMPUTER CODE

ADAPTED FROM THE BNHL PROGRAH COBRA-IV-I NITH UPOATES THROUGH FX 15 OATEO O62480.

COBRA DIMENSION PARAMETERS

\begin{tabular}{|c|c|c|c|}
\hline $\begin{array}{l}\text { MA } \\
\text { M.C }\end{array}$ & $\begin{array}{l}28 \\
28\end{array}$ & $\begin{array}{l}\text { SURCHANNELS WITH AREA VARIATIONS } \\
\text { SUBCHANNELS }\end{array}$ & $\begin{array}{l}\text { GRP } \\
\text { GRP }\end{array}$ \\
\hline M.E & 30 & $1=M \times I$ & GFP \\
\hline MG & 41 & SUECHANNEL GAP CONNECTIONS & GRP \\
\hline MI & 7 & CONNECTIONS TO A CHANNEL (THE RMAL \&LOH). & GRP \\
\hline MK & 6. & GRIO SPACFR TYPES & GRP \\
\hline ML & 6 & AXIAL LOCATIONS FOR GAP AREA VARIATIONS & GRP \\
\hline MO & 7 & WIDTH OF AAA ARRAYITRIANGLE $=5$, HANGAP: $=71$ & GRP \\
\hline M.P & 20 & CAROS IN COOLANT PROPERTY TABLE. & GRP \\
\hline MR & 8 & ROOS & GRP \\
\hline MS & 39 & GAPS WITH GAP SPACING VARIATIONS & GRP \\
\hline MT & 9 & CORRELATIONS & GRP \\
\hline MW & 12 & THERMAL CONNECTIONS & GRP \\
\hline$M x$ & 30 & AXIAL NODES PLUS ONE & GRP \\
\hline MY & 5 & RAOIAL ROD OIVISIONS & GRP \\
\hline M. 2 & 12 & AXIAL LOCATIONS FOR GRIO SPACERS & GRP \\
\hline NU & 55 & SURFACES & GRP \\
\hline
\end{tabular}

AXIAL LOCATIONS FOR GRIO SPACERS 


\begin{tabular}{|c|c|c|c|c|}
\hline \multicolumn{5}{|c|}{ INPUT FOR CASE } \\
\hline SUMMARY & $Y D F$ & INPUT & OPTI & NS \\
\hline GROUP & vI & N2 & $\mathrm{N3}$ & $\mathrm{N}_{4}$ \\
\hline 1 & 20 & 0 & 0 & 0 \\
\hline 2 & D & c & 0 & 0 \\
\hline 3 & 15 & 0 & $c$ & 0 \\
\hline 4 & 27 & 27 & 0 & 0 \\
\hline 5 & 27 & 6 & 0 & 0 \\
\hline 7 & 2 & 0 & 12 & 2 \\
\hline 8 & 8 & 8 & 0 & 0 \\
\hline 9 & 0 & c & 0 & 0 \\
\hline 10 & 4 & G & 0 & 0 \\
\hline 11 & 1 & 0 & 0 & 0 \\
\hline 12 & 13 & 0 & 0 & 0 \\
\hline 13 & 0 & 0 & 0 & 0 \\
\hline
\end{tabular}

HELIUM PROPERTIES AT 9000.JO KPA

T $H$ H TOODO KO KP

$\begin{array}{ccc}T & H & C F \\ D E G C & K J / K G & K J / K G-K \\ 324.0 & 3135.7 & 5.188 \\ 332.4 & 3179.0 & 5.188 \\ 340.7 & 3222.3 & 5.188 \\ 349.1 & 3265.7 & 5.138 \\ 374.1 & 3395.7 & 5.188 \\ 399.2 & 3525.6 & 5.188 \\ 424.2 & 3655.6 & 5.187 \\ 449.3 & 3785.6 & 5.187 \\ 474.4 & 3915.6 & 5.187 \\ 499.4 & 4045.6 & 5.187 \\ 524.5 & 4175.6 & 5.187 \\ 549.5 & 4305.6 & 5.187 \\ 574.6 & 4435.6 & 5.187 \\ 599.6 & 4565.6 & 5.187 \\ 624.7 & 4695.6 & 5.187 \\ 649.8 & 4825.6 & 5.187 \\ 674.8 & 4955.5 & 5.188 \\ 699.9 & 5085.5 & 5.188 \\ 724.9 & 5215.5 & 5.188 \\ 750.0 & 5345.5 & 5.188\end{array}$

$V$
$C U / K G$
.1405
.1424
.1443
.1462
.1520
.1578
.1635
.1693
.1750
.1808
.1866
.1923
.1981
.2039
.2096
.2154
.2212
.2269
.2327
.2385

$K$
$K W / M-K$

.000245

.000247

.000250

.000252

.000259

.000266

.000273

.000280

.000287

.000293

.000300

.000307

.000313

.000313

.000320

.000326
.000332

.000339

.000345

.0 .00351

.674

.674

.673

.673

.673

.0000406

.0000422

.0000431

.0000446

.0000454

$.0 n 004 B 2$

FRICTION FACTOR COFRELATION

$$
\text { SMOOTH SECTION }
$$

CHANAEL TYPE I FRICT $=.52000+00 \% R E \$ 1-.32000001+.58240-02$ CHANNEL TYPE 2 FRICT $=.52000+00$ RE\$1-.3200+001 \$.58240-02 CHANNEL TYPE 3 FRICT $=.52000+00 \%$ RE $\$ 1-.3200+001, .58240-02$ CHANPJEL TYPE 4 FRICT $=.52000+00 \%$ RE \$1-.3200+00)+.58240-02 CHANHEL TYPE 5 FRICT $=.52000+D 0$ RE \$1-.3200*001+.58240-02

STANTON NUMBER CORRELATIDN

$$
\text { SMOOTH SECTION }
$$

CHANVEL TYPE $1-5$ STN = PETUKHOV-ROIZEN METHOO

$.000357 \quad .671$

HEAT FLUX DISTRIEUTION

$\begin{array}{lcl}X / L & \text { RELATIVE FLUX . BEGINNIHS OF HEATING }=938.94 \mathrm{MM} \\ .0000 & .0330 & \end{array}$

CHANNEL TYPE 1 FRICT= CHANHEL TYPE 2 FRICT= CHANNEL TYPE 3 FRICT= CHANNEL TYPE 4 FRICT= CHANNEL TYPE 5 FPICT=
ROUGH SECTION

R -FUNCTION CORRELATION R-FUNCTION CORRELATION R-FUNCTION CORRELATION R-FUNCTION CORRELATION R-FUNCTION CORRELATION

ROUGH SECTION

CHANNEL TYPE $1-5$ STN = G-FUNCTION CORRELATION 


$\begin{array}{ll}.3287 & .4980 \\ .3670 & .6560 \\ .4100 & .8000 \\ .4520 & .9080 \\ .4940 & .9760 \\ .5380 & 1.0000 \\ .5790 & .9800 \\ .6210 & .9150 \\ .6640 & .8100 \\ .7060 & .6690 \\ .7480 & .4980 \\ .7481 & .0000 \\ 1.0000 & .0000\end{array}$

SUBCHANNEL INPUT DATA

CHANNEL TYPE

ISO-AMI HETTED

(MM)

19.10

11.48

22.90

38.26

19.10

19.10

38.26

$38 \cdot 26$

38.26

22.90

11.48

6.39

24.77

24.77

12.45

24.77

21.87

24.77

12.45

24.77

24.77

6.06

12.13

12.13

6.15

6.15
4.10

8.20

12.31

2.31

6.15

12.31

12.31

$12 \cdot 31$

4.10

2.05

10.10

10.10

$4 \cdot 10$
10.10

12.40

10.10

$4 \cdot 10$

10.10

10.10

2. 55

2.47

4.94

4.94
2.47
HYDRAULIC

DIAMETER

(MM)

12.423
12.423
11.200
11.171
12.432
12.423
12.423
12.432
12.432
12.432
11.171
11.200
12.468
9.810
9.810
12.146
9.810
7.055
9.810
12.146
9.810
9.810
12.468
9.814
9.822
9.822
9.814

IADJACENT CHANNEL NO., SFACING, CENTROIO DISTANCEI

12,4

$2,4.170,4.320116,2.080,6.86011$ 3.2 .080 .6 .86011
$4,1.680,5.59011$
$5,1.3 .400, .00011$ $5,4.170,6.8501111,1.680,6.86011$ $6,4.170 .6 .100118,4.170,6.86011$ $.0,4.170 .6 .100110,4.170,6.86011$ $8,4.170,6.1001123,2.080,4.57011$ $8,4.170 .6 .1001123,2.080,4.57011$
$9,4.170 .6 .86011$
$1, .000, .00011$ $10,4 \cdot 170.6 .8501120,4 \cdot 170,5.08011$

$11,4.170 .6 .8301116,4.170,5.08011$

$12,1.680,6.1001126,6.930,2.29011$

$13,2.080,4.5701127,3.730,2.54011$

$14,3.020,3.810110, .000, .00011$

$15,3.280 .6 .100110, .000, .00011$

$16,3.020 .4 .0601117,3.730,7.11011$

$17,3.020 .4 .060110, .000, .00011$

$18,3.280,6.300110, .000, .00011$

$19,3.280,6.100110, .000, .00011$

$20,3.020,4.0501121,3.730,7.11011$

$21,3.020,4.060110, .000, .00011$

$22,3.280,5.100110, .000, .00011$

$23,3.020,3.810110, .000, .00011$

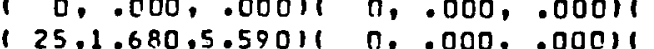

$26,1.680,5.590110, .000, .00011$

$27.1 .680 .5 .590110, .000, .00011$

$10, .000, .000110, .000, .00011$

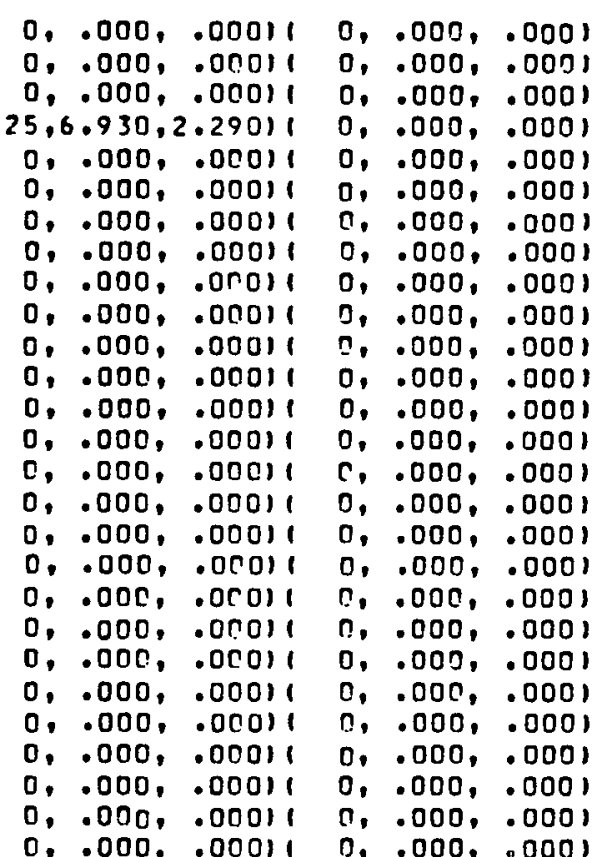

$0, .000, .000110, .000, .0001$ $0.000, .0001$ $0, .0001$ $0, .000, .0001$ 0.000 $.000, .0001$ $.000, .0001$ 0001 $0, .000, .0001$ $0, .000, .0001$ $0, .000, .0001$ $.000, .0001$ $, .000, .0001$ $0.000, .0001$ $0, .000, .0001$ $0.000, .0001$ $.000, .0001$

$X / L$ AREA VARIATION FACTORS FOR SUBCHANNEL III

\begin{tabular}{|c|c|c|c|c|c|c|c|c|c|c|}
\hline & & & & & & & & & & \\
\hline & 11 & 21 & 31 & 41 & 51 & 61 & 71 & 81 & 91 & $(10)$ \\
\hline .0000 & .9730 & .9730 & .9670 & .9670 & .9730 & .9730 & .973[ & .9730 & .9730 & .9730 \\
\hline 248 & .9730 & .9730 & .9670 & .9670 & .9730 & .9730 & $.97 \geq 0$ & .9730 & .9730 & .9730 \\
\hline .3252 & 1.0000 & 1.0000 & 1.0000 & 1.0050 & 1.0000 & 1.0000 & 1.0000 & 1.0000 & 1.0000 & 1.0000 \\
\hline .7518 & 1.0000 & 1.0000 & 1.0000 & $1.00 n 0$ & 1.0000 & 1.0000 & 1.0000 & 1.0000 & 1.0000 & 1.0000 \\
\hline .7521 & .9730 & .0730 & .9670 & .9670 & .9730 & .9730 & $.973 \pi$ & .0730 & .9730 & ? \\
\hline $.0 C 00$ & .9730 & .9730 & .9670 & .9670 & .9730 & .9730 & .9730 & .9730 & .9730 & .9730 \\
\hline (1 & AREA & ATION & CTORS & SU & & & & & & \\
\hline & 1111 & 1121 & 131 & 141 & $(15)$ & $(16)$ & 1271 & 1281 & 1191 & 1201 \\
\hline
\end{tabular}




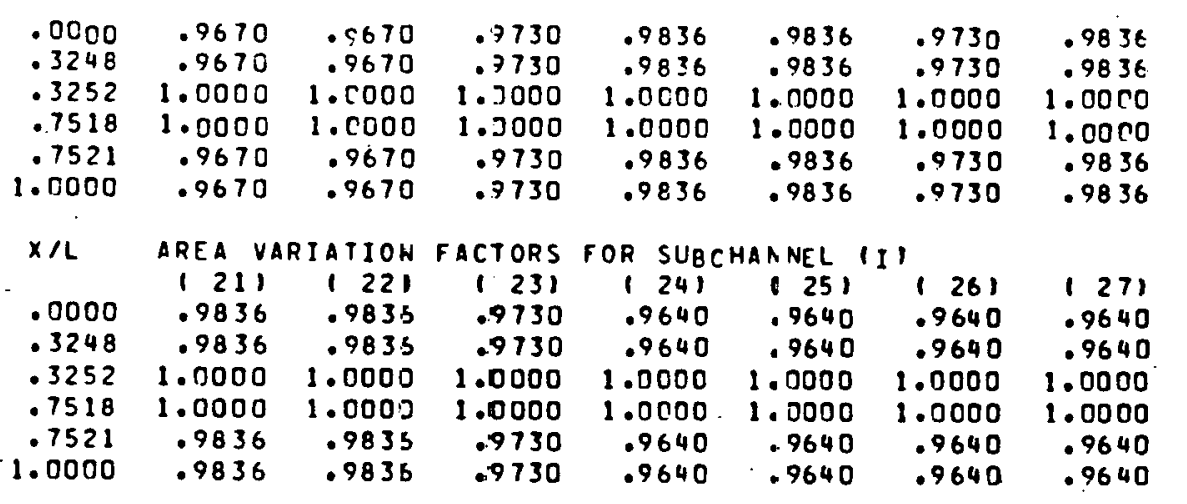

SPACER DATA

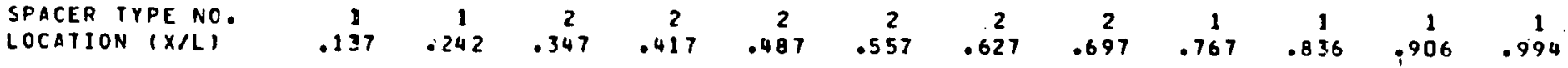

SPACER LOSS COEFFICIENT

SPACER IYPE 1 COEF $=$ CO* $\$ .20000+01 * 1.51400+01+.82800+03 / R E * .50000+C 01$

SPACER TYPE 2 COEF = CO\& .20000+01*1.38200+01:.76100+03/RE\$.50000+001

i SPACER HIDTH $=15.00 \mathrm{MM}$

HEAT TRANSFER COEFFICIENT ENHANCEMENT DUE TO SPACERS

ROUGH BUNOLE - YST $=1+3.350 * C D * 2 * F(X)$

SMOOTH BUNDLE - YST $=1+5.550 * C D * 2 * F(x)$

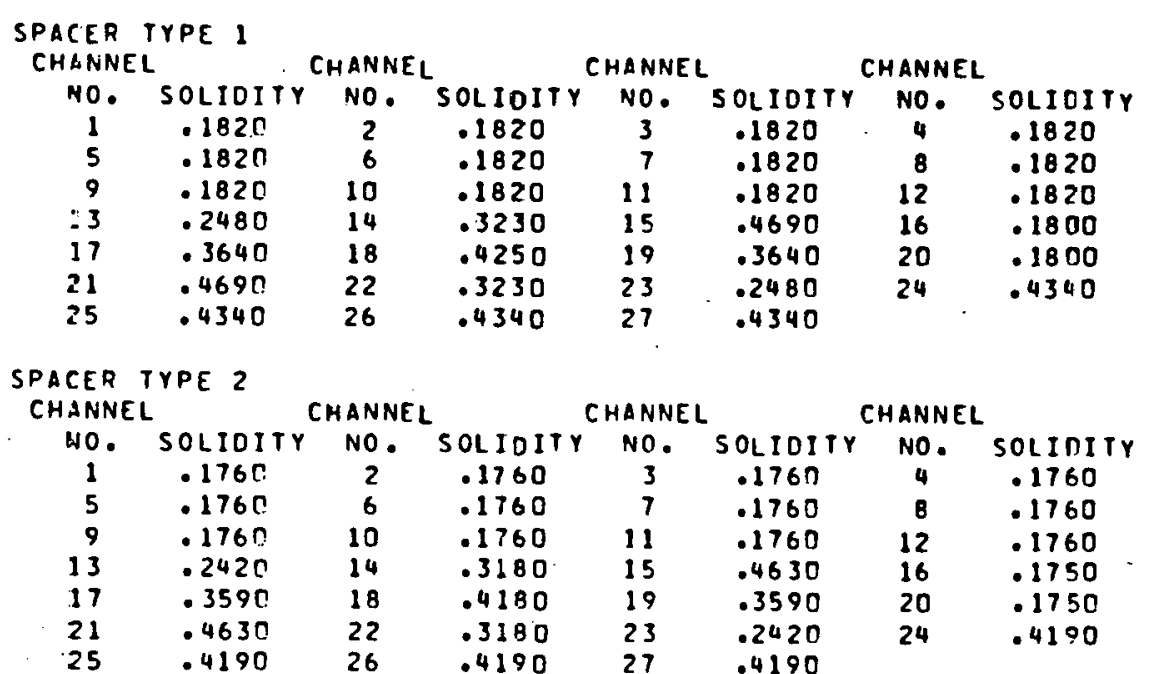

ROD INPUT OATA

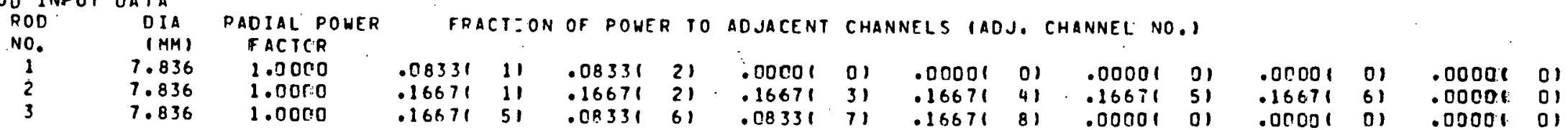




$\begin{array}{lll}4 & 9.436 & .0000 \\ 5 & 7.836 & 1.0000 \\ 6 & 7.836 & 1.0000 \\ 7 & 7.836 & 1.0000 \\ 8 & 7.836 & 1.0000\end{array}$

$.08331241 \quad .16671251$ .1667141 .16671 .51 .1667171 .1667181 .1667191 .16671101

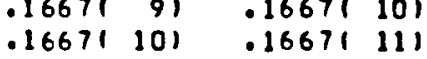

.16671261 .1667181 .1567191 .08331161 .15621121 .1667191 .08331201 .1667117 .08331131

.000010 .16671101 .16671211 .16671181 .0000101 .0000101 .16671111 .0000101 .16671 .221 .08331231 $.16671 .191 \quad .08331201$ IMPLICIT SOLUTION WITH INLET FLOWS SPECIFIEO

$\begin{array}{ll}\text { CALCULATION PARAMETERS } & \\ \text { LATERAL RESISTANCE FACTOR } & .6000 \\ \text { ISLLI PARAMETER } & .5000 \\ \text { IURBULENT MOMENTUM FACTOR } & 1.0000\end{array}$

CHANNEL ORIENTATION

. NOOO DEGREES

DATA FOR IMPLICIT SOLUTION EXTERNAL ITERATION LIMIT INTERNAL ITERATION LIMIT CONVERGENCE FACTORS

$$
\text { EXTERNAL (DW/H) }
$$$$
\text { FLOW }
$$

$$
\text { IDF/FI }
$$

$\triangle X I A L$ NOOE LOCATIONS IMMI

$\begin{array}{rr}.0 & 186.6 \\ 1391.2 & 1472.0 \\ 2402.8 & 2544.2\end{array}$

$$
\begin{array}{r}
186.6 \\
1472.0 \\
2544.2
\end{array}
$$

20
72
.0500
.0010
.0100

CHANNEL LENGTH NUMBER OF AXIAL NODES

ROUGHENING BEGINS ROUGHENING ENDS

TOTAL TRANSIENT TIME

NUMBER OF TIME STEPS

NOMINAL TIME STEP.

MINIMUM INTERNAL ITERATIONS

FRACTION DONOR CELL USTAR

ACCELEPATION FACTORS

CFOSSFLOW SOLUTION

LATERAL DELTA -P

FLOH

$16671 \cdot 151$

MIXING CORRELATIONS

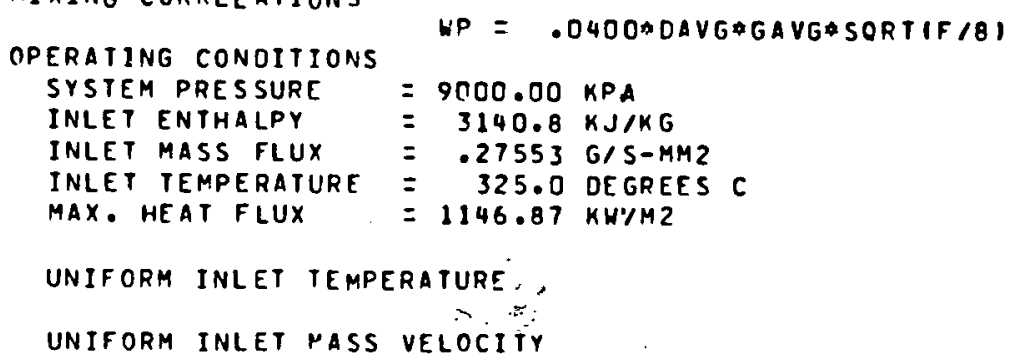

\begin{tabular}{|c|c|c|c|c|}
\hline 1 & 2 & 3 & 4 & 5 \\
\hline .000 & 8.595 & 7.516 & .000 & .000 \\
\hline $6.30 n$ & . & $.00 \%$ & 6.081 & 6.721 \\
\hline 3.918 & 3.918 & 7.918 & 3.918 & 4.71 \\
\hline
\end{tabular}




\section{FULLY ROUGH FLOW $\left(H^{+}\right) 50.1: \quad R(H+)=4.37$} TRANSITION REGION:

HYDRAULICALLY SMOOTH: $R(H+1)=5.23-.2180 \mathrm{LN}(\mathrm{H}+)$ $R(H+)=5.5+2.5(N(H B+)$

$G(H H+1=6.27 * 1 H H+1 * .25 * P R \# .44 *(T H / T B) * .35$ $F O Z H+R 30.0 \mathrm{G}(H \mathrm{HH})=13.64$
SLOPE $A=2.00$

$A=6.209-2.0070 \mathrm{LN}(\mathrm{H}+)+.2380 \mathrm{LN}(\mathrm{H}+1) * 2 * 2$

$A=2.50$ 
DATA =ROM ITERATIVE SOLUTION

\begin{tabular}{|c|c|c|c|c|c|c|c|c|}
\hline \multirow[t]{2}{*}{$\begin{array}{l}\text { ITERATION } \\
\text { NO. }\end{array}$} & \multirow{2}{*}{$\begin{array}{c}\text { TOTAL INTERNA: } \\
\text { ITERATIONS } \\
\text {. }\end{array}$} & LAST & $\begin{array}{l}\text { NODE OUT } \\
\text { IVERGENCE }\end{array}$ & \multirow[t]{2}{*}{$\begin{array}{l}\text { MAX IMUM } \\
\text { INTERNAL }\end{array}$} & \multirow[t]{2}{*}{$\begin{array}{l}\text { ERPOP } \\
\text { EXTERNAL }\end{array}$} & \multirow[t]{2}{*}{ FLOW } & \multirow[t]{2}{*}{ ENTHALPY } & \multirow[t]{2}{*}{ TIME } \\
\hline & & H & $\mathbf{F}$ & & & & & \\
\hline 1 & $14 i 2$ & 25 & 25 & 333.7152 & .9962 & .1535 & .0609 & 21 \\
\hline 2 & 1139 & 25 & 25 & 24.1977 & .7945 & .0740 & .0529 & 39 \\
\hline 3 & 1440 & 25 & 25 & 7.5051 & .5706 & .0425 & .0036 & 58 \\
\hline 4 & .1684 & 25 & 25. & 6.6605 & .1422 & .0230 & .0015 & 77 \\
\hline 5 & 1679 & 24 & 25 & 5.1748 & .1212 & .0130 & .0013 & 96 \\
\hline 6 & 1675 & 23 & o & 3.4874 & .1030 & .0082 & .0009 & 115 \\
\hline 7 & 1618 & 23 & 0 & 4.3545 & .0935 & .0057 & .0009 & 134 \\
\hline 8 & 1594 & 23 & a & 2.2465 & .0830 & .0039 & .0008 & 153 \\
\hline 9 & 1594 & 23 & o. & 2.9870 & .0718 & .0026 & .0008 & 171 \\
\hline 10 & 1594 & 23 & 0 & 4.1880 & .0616 & .0018 & .0010 & 190 \\
\hline 11 & 1594 & 23 & 0 & 4.5777 & .0565 & .0016 & .0012 & 209 \\
\hline 12 & 1594 & 18 & 0 & 2.5438 & .0520 & .0017 & .0010 & 228 \\
\hline 13 & 1529 & 0 & 0 & .1717 & .0477 & .0016 & .0009 & 246 \\
\hline
\end{tabular}


CHANNEL EXIT SUMMARY RESULTS

CASE 1 CFTL BUNDLE - ONE SIXTH SECTION - FULLY POUGH FLOH - 1113181

MASS BALANCE -
MASS FLOW IN

MASS FLOW OUT

$$
.14581 \text { KG/SEC }
$$

ENERGY BALANCE -

FLOW ENERGY IN

ENERGY ADOED

FLOW ENERGY OUT

ENERGY ERROR

$457.95291 K H$

$163.13234 \mathrm{KW}$

$621.08381 \mathrm{KH}$

$. .14305-02 \mathrm{KH}$ OR -.001 PCT.

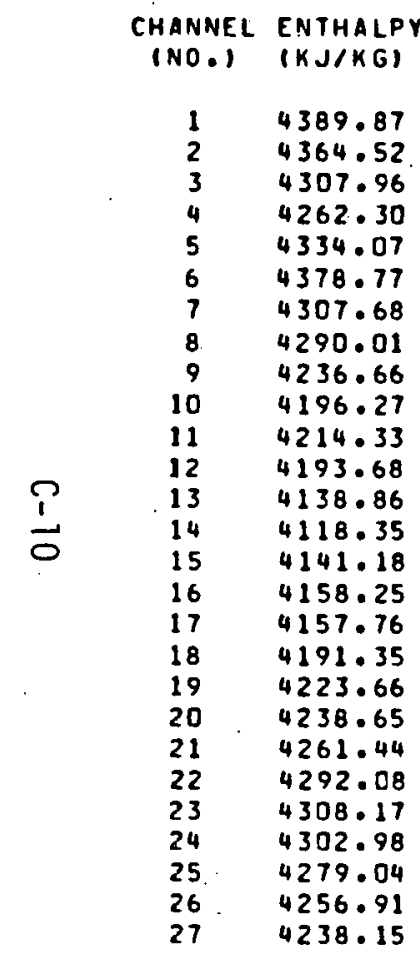

TEMPERATU
IOEG-CI
565.9
561.0
550.1
541.3
555.1
563.7
550.0
546.6
536.3
528.5
532.0
528.0
517.5
513.5
517.9
521.2
521.1
527.6
533.8
536.7
541.1
547.0
550.1
549.1
544.5
540.2
536.6

FLOW

Mass FLUX

(G/S-MM2)

5.027

.3247

$\begin{array}{ll}.00620 & .3247 \\ .00614 & .3215\end{array}$

00347

$\begin{array}{lll}5.122 & .00347 & .3022 \\ 5.177 & .00689 & .3009 \\ 5.091 & .01240 & .3242\end{array}$

$\begin{array}{lll}5.122 & .00347 & .3022 \\ 5.177 & .00689 & .3009 \\ 5.091 & .01240 & .3242\end{array}$

$\begin{array}{lll}5.091 & .01240 & .3242 \\ 5.040 & .00621 & .3254\end{array}$

$5.122 \quad .00627 \quad .3283$

$5.143 \quad .01251 .03270$

$5.208 \quad .01218 \quad .3184$

$5.257 \quad .01198 \quad .3131$

.00652

$5.330 \quad .00163 \quad .2561$

$00163 \quad .2549$

$.00554 \quad .2238$

$.00453 \quad .1827$

$\begin{array}{lll}5.305 & .00320 & .2574 \\ 5.306 & .00470 & .1896\end{array}$

$5.264 \quad .00378$

$5.224 \quad .00523$

$5.205 \quad .00335$

$5.178 \quad .00453$

5.1410 .00628

$5.122 \quad .00203$

$.00250 \quad .2064$

$\begin{array}{lll}5.183 & .00235 & .1937 \\ 5.206 & .00110 & .1822\end{array}$ 
CHANNEL RESULTS

EASE. 1 CFTL EUNDLE - ONE SIXTH SECTION - FULLY ROUGH FLOH - 1/13/81

BUNDLE AVERAGED RESULTS

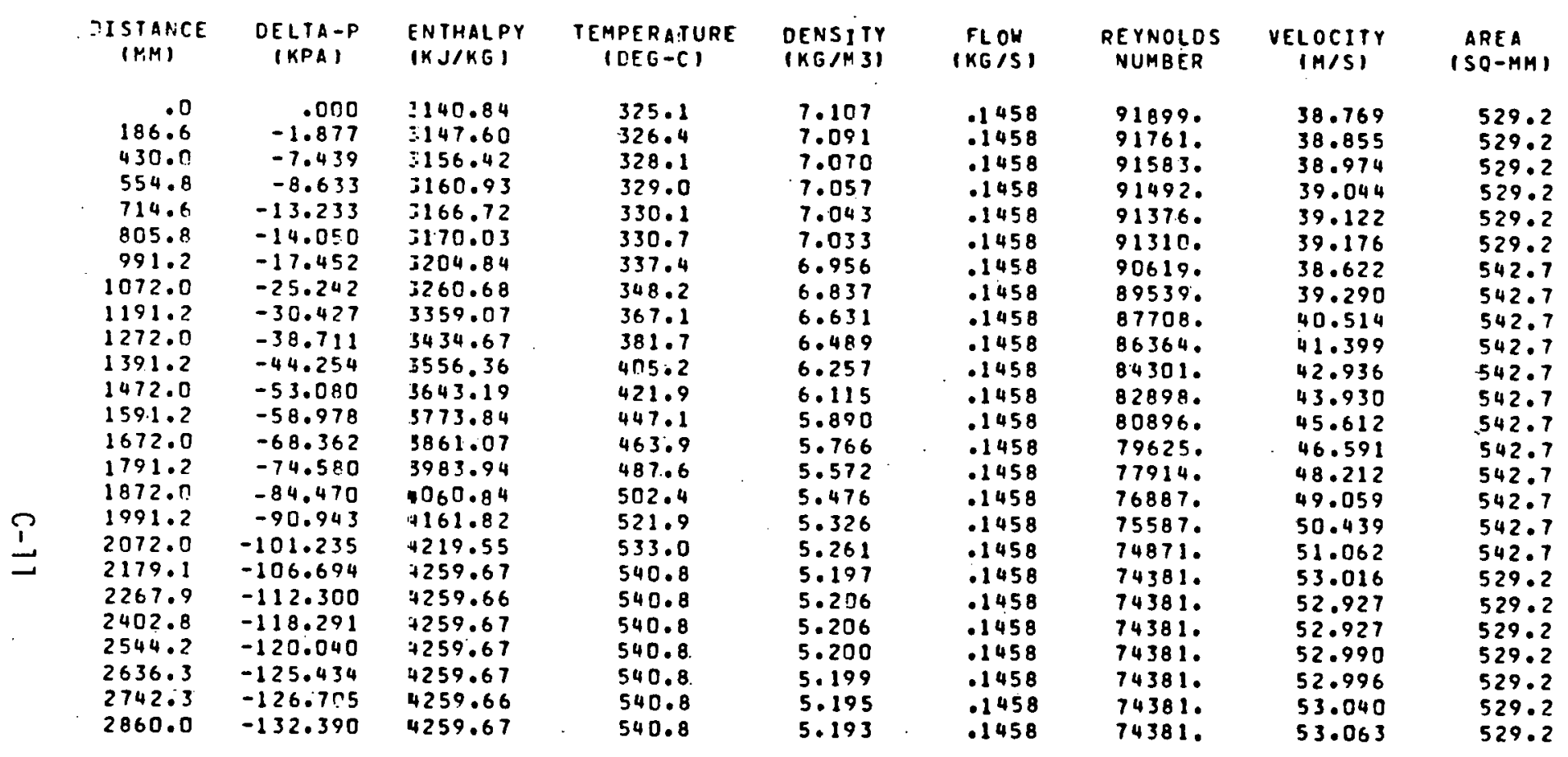


CHANNEL RESULTS
CASE 1

OSTE 27 MAY 81

IIME 11:21:28

IIME $=.00000$ SECONDS PRESSURE $=9000.0 \mathrm{KPA}$

DISTANCE DELTA-P TEMP (MM)

$\begin{array}{rrr}.0 & \text { (KPA) } \\ 186.0 & -1.000\end{array}$

$186.6-1.879$

186.6
430.0

554.8

554.8

805.8

991.2
1072.0

1191.2

1272.0

1391.2

1472.0

1591.2

1672.0
1791.2

1872.0

1991.2

$2072 . n$

2179.1

2402.8

2402.8
2544.2

2636.3

2742.3

$-1.430$

$-8.639$

$-13.218$

$-14.047$

-17.474
-25.201

$-30.496$

$-38.653$

$-44.318$

$-53.015$

$-59.047$

$-68.292$

$-74.654$

-84.397
-91.022

$-101.157$

$-106.785$

$-112.260$

$-118.268$

$-120.073$

$-125.401$

$-126.726$

TEMPER ATURE

OEG-C) OENSITY

325.1

326.6

328.7
329.7

330.9

331.5

338.8

351.2

372.3

389.9

416.6

437.0

465.6

485.9

512.6

530.2

551.8

564.4

572.3

571.3

570.0

568.9

567.9

567.0

565.9

7.107

7.061

7.047

7.031

7.021

6.800

6.577

6.401

6.154

5.976

5.744

5.588

5.397

5.276

5.135

5.004

5.006

5.011

5.015

5.029

5.022
5.027
DATA FOR CHANNEL I

$\begin{array}{ccc}\text { REYNOLDS } & \text { AREA } & \text { FRICIION } \\ \text { NUMBER } & \text { IMM21 } & \text { FACIOR } \\ 102448 . & 18.58 & .01878 \\ 104165 . & 18.58 & .01872 \\ 114914 . & 18.58 & .01832 \\ 113624 . & 18.58 & .01836 \\ 120103 . & 18.58 & .01814 \\ 118105 . & 18.58 & .01821 \\ 111492 . & 19.10 & .04911 \\ 113812 . & 19.10 & .10808 \\ 101694 . & 19.10 & .10761 \\ 106415 . & 19.10 & .10698 \\ 95666 . & 19.10 & .10693 \\ 100641 . & 19.10 & .10666 \\ 90684 . & 19.10 & .10696 \\ 95702 . & 19.10 & .10701 \\ 86621 . & 19.10 & .10756 \\ 91798 . & 19.10 & .10788 \\ 83637 . & 19.10 & .10857 \\ 89102 . & 19.10 & .10910 \\ 81868 . & 19.58 & .08494 \\ 90347 . & 18.58 & .01932 \\ 95145 . & 18.58 & .01910 \\ 92913 . & 18.58 & .01920 \\ 97153 . & 18.58 & .01901 \\ 95696 . & 19.58 & .01910 \\ 98347 . & 18.58 & .01896\end{array}$

18.58

$\begin{array}{ll}\text { STANTON } & \text { FILMH } \\ \text { NUMBER } & \text { KHIM2-C } \\ .00295 & 4.221 \\ .00294 & 4.281 \\ .00287 & 4.625 \\ .00288 & 4.589 \\ .00284 & 4.865 \\ .00285 & 4.735 \\ .00535 & 8.243 \\ .00536 & 7.853 \\ .00549 & 8.056 \\ .00545 & 7.808 \\ .00557 & 8.066 \\ .00551 & 7.856 \\ .00563 & 8.121 \\ .00557 & 7.911 \\ .00568 & 8.163 \\ .00561 & 7.954 \\ .00571 & 8.200 \\ .00563 & 7.979 \\ .00291 & 4.321 \\ .00284 & 4.542 \\ .00281 & 4.930 \\ .00283 & 4.634 \\ .00280 & 4.793 \\ .00281 & 4.712 \\ .00279 & 4.952 \\ & \end{array}$

H

KG/M3) FLOW

IKG/SI
.005121
.005216
.005767
.005709
.006043
.005947
.005565
.005759
.005265
.005612
.005184
.005564
.005151
.005539
.005134
.005524
.005125
.005517
.005190
.005722
.006020
.005873
.006136
.006002
.006201

98347 .
$R(H+)$

.0

$\begin{array}{lll}.0 & .00 & .0 \\ .0 & .00 & .0\end{array}$


CHANNEL RESULTS

CASE 1 CFTL BUNOLE - ONE SIXTH SECTION - FULLY ROUGH FLON - 1/13/81

TIME $=.00000$ SECONOS PRESSURE $=9000.0 \mathrm{KPA}$

\begin{tabular}{|c|c|c|c|c|}
\hline $\begin{array}{c}\text { DISTANCE } \\
\text { (MM) }\end{array}$ & $\begin{array}{l}\text { DELTA-P } \\
\text { (KPA) }\end{array}$ & $\begin{array}{l}\text { TEMPER ATURE } \\
\text { (DEG -C) }\end{array}$ & $\begin{array}{l}\text { DENSITY } \\
\text { (KG/M } 3 \text { ) }\end{array}$ & $\begin{array}{l}F L O W \\
\text { (KG) }\end{array}$ \\
\hline $\begin{array}{r}.0 \\
186.6\end{array}$ & $\begin{array}{r}.000 \\
-1.879\end{array}$ & $\begin{array}{l}325.1 \\
326.6\end{array}$ & $\begin{array}{l}7.107 \\
7.087\end{array}$ & $\begin{array}{l}.005121 \\
.005205\end{array}$ \\
\hline 430.0 & -7.432 & 328.6 & 7.062 & .005712 \\
\hline 554.8 & -8.638 & 329.6 & 7.048 & .005642 \\
\hline 714.6 & -13.220 & 330.7 & 7.033 & .005957 \\
\hline 805.8 & -14.047 & 331.4 & 7.023 & .005855 \\
\hline 991.2 & -17.472 & 338.7 & 6.940 & .005475 \\
\hline 1072.0 & -25.205 & 351.0 & 6.801 & .005684 \\
\hline 1191.2 & -30.482 & 372.1 & 6.579 & .005199 \\
\hline 1272.0 & -38.658 & 389.3 & 6.407 & .005548 \\
\hline 1391.2 & -44.314 & 415.8 & 6.161 & .005125 \\
\hline 1472.0 & -53.020 & 435.5 & 5.988 & .005506 \\
\hline 1591.2 & -59.043 & 464.0 & 5.756 & .005097 \\
\hline 1672.0 & -68.298 & 483.5 & 5.606 & .005485 \\
\hline 1791.2 & -74.650 & 510.2 & 5.413 & .005083 \\
\hline 1872.0 & -84.403 & 526.9 & 5.297 & .005474 \\
\hline 1991.2 & -91.017 & 548.6 & 5.155 & .005077 \\
\hline 2072.0 & -101.164 & 560.5 & 5.078 & .005470 \\
\hline 2179.1 & -106.779 & 568.6 & 5.026 & .005143 \\
\hline & 2.265 & 566.5 & .034 & .005669 \\
\hline 2402.8 & -118.272 & 565.0 & 5.040 & .005965 \\
\hline 2544.2 & -120.070 & 564.2 & 5.043 & .005814 \\
\hline 2636.3 & -125.407 & 562.8 & 5.049 & .006080 \\
\hline 2742.3 & -126.725 & 562.3 & 5.050 & .005940 \\
\hline 2860.0 & -132.390 & $561: 0$ & 5.056 & .006141 \\
\hline
\end{tabular}

DATA FOR CHANNEL

$\begin{array}{lll}\text { PEYNOLDS } & \text { AREA } & \text { FRICTION } \\ \text { NUHBER } & \text { IMM2I } & \text { FACTOR } \\ 102448 . & 18.58 & .01878 \\ 103956 . & 19.58 & .01872 \\ 113823 . & 18.58 & .01836 \\ 112297 . & 18.58 & .01841 \\ 118422 . & 18.58 & .01820 \\ 116292 . & 18.58 & .01827 \\ 109702 . & 19.10 & .04915 \\ 112345 . & 19.10 & .10804 \\ 100436 . & 19.10 & .10757 \\ 105263 . & 19.10 & .10693 \\ 94651 . & 19.10 & .10688 \\ 99732 . & 19.10 & .10661 \\ 89870 . & 19.10 & .10690 \\ 94987 . & 19.10 & .10696 \\ 85955 . & 19.10 & .10751 \\ 91222 . & 19.10 & .10783 \\ 83071 . & 19.10 & .10853 \\ 88619 . & 19.10 & .10906 \\ 81386 . & 18.58 & .08493 \\ 89849 . & 18.58 & .01934 \\ 94666 . & 18.58 & .01912 \\ 92325 . & 18.58 & .01922 \\ 96658 . & 18.58 & .01903 \\ 94483 . & 18.58 & .01913 \\ 97783 . & 18.58 & .01898\end{array}$

FILM H

0029

00288

.00289

.00285

00286

.00537

00546

.00558

00562

00572

.00564

.03291

00282

.00280
KHIMZ - C

4.221
4.274

4.591

4.547

4.812

4.678

8.144

7.778

7.981

7.742

7.997

7.793

8.056

7.851

8.099

7.896

8.138

7.922

4.292

4.510

4.896

4.599

4.760

4.676

4.916
$R(H+)$

.0
.0

.0
.0
.0

.0

27.4

110.0

95.9

97.1

87.1

90.4

82.8

81.8

81.8

88.3

83.5

91.6
84.7

4.7
.0

.0
.0
.0
.0

.0

.0
.00
.00
.00
.00
.00
.00
4.37
4.37
4.37
4.37
4.37
4.37
4.37
4.37
4.37
4.37
4.37
4.37
4.37
.00
.00
.00
.00
.00
.00
.0

.0

.0

.0

.0

2.0

2.0

2. 0

2.0

2.1

2.0

2.0

2.0

2.0

2.0

2.0

2.0

.0

.0

.0

.0 
CHANNEL RESULTS

CASE 1 CFTL BUNOLE - ONE SIXTH SECTION - FULLY ROUGH FLOH - 1/13/81

TIME $=.00000$ SECONDS PRESSURE $=9000.0 \mathrm{KPA}$

DISTANCE DELTA-P

IMM

(MM)

186.6

430.0

554.8

714.6

805.8

1072.0

191.2

1272.0

1391.2

1472.0

1591.2

1672.0

1791.2

1872.0

1991.2

ELTA

.000

$-1.878$

$-7.434$

$-8.637$

-13.225
-14.047

$-17.466$

$-25.215$

$-30.471$

$-38.670$

$-44.302$

$-53.034$

$-59.030$

$-68.313$

$-74.636$

$-84.419$

$-91.003$

$-101.180$

$-106.762$

2179.1

$-112.276$

$2402.8 \quad-118.278$

$2544.2 \quad-120.063$

$2636.3 \quad-125.418$

$2742.3 \quad-126.721$

2860.0
TEMPERATURE DENSITY

IXG/M3I FLOW

7.107

328.3

330.3

331.0

338.2

349.9

370.8

386.3

412.7

430.6

459.2

476.8

503.8

518.8

541.0

551.4

560.0

556.5

553.6

553.2

55.

551.

551.6

550.1

.003059

.003065

.003233

00331

003067

7.038

7.028

6.945

6.813

6.503 .

6.029

5.793

5.655

5.457

5.350

5.203

5.133

5.076

5.094

5.109

5.109

5.116

5.115

5.122

002907

.002875

.003084

. 002867

.003079

.002864

.003077

.002863

.003078

.002888

.003183

.003378

.003302

.003433

.003364

.003253

003098

.003469

DATA FOR CHANNEL 3

PEYNOS AREA FRICTION

O1794. 11.10

91794. 11.10 .01925

$96677 . \quad 11.10 \quad .01925$

$95033.11 .10 \quad 01910$

$98780 . \quad 11.10 \quad 001894$

$96961 . \quad 11.10 \quad .01902$

$92224.11 .48 \quad .05159$

$94045.11 .48 \quad .11369$

$84354 . \quad 11.48 \quad .11319$

$11.48 \quad .11248$

$79887 . \quad 11.48 \quad .11245$

$84194 . \quad 11.48 \quad .11212$

$76160 . \quad 11.48 \quad .11247$

$80454 . \quad 11.48 \quad .11249$

$73043 . \quad 11.48 \quad .11313$

$77447.11 .48 \quad .11343$

$70730 . \quad 11.48 \quad .1142$

$75375 . \quad 11.48 \quad .11477$

$69036.11 .10 \quad .08929$

76310. $11.10 \quad .02007$

$81165.11 .10 \quad .01979$

$79383.11 .10 \quad .01989$

$82616.11 .10 \quad .0197$

80962. $11.10 \quad .01980$

83605. $11.10 \quad .01966$ STANTON

.00300

H* $R(H+)$

AR

$.00300 \quad 4.290$

4.296

.002964 .476

00295

4.421

.00296400

00559

4.496

.00560

7.923
7.533

7.752

7.501

7.784

7.562

7.562

7.856

7.625
7.906

7.673

7.949

7.702

4. 144

4.372

4.755

4.498

4.635

0028

4.563

4.761

.00288

0
123.
105.
92.
93.5
84.
80.
85.
79.
86.0
89.6
82.6
.0
.0

.0
.0
.0
.0
.0
.0
23.8
05.8
92.5
93.5
84.4
87.4
80.5
85.2
79.9
86.0
81.8
89.6
82.6
.0
.0
.0
.0
.0
.0

.00
.00

.00

.00

.00

.00
4.37

4.37

4.37

4.37

4.37

4.37

4.37

4.37

4.37

4.37

4.37

4.37

.00

.00

.00
.00

.00
.00
.00

.0
.0
.0
.0
.0
.0
2.0
2.0
2.0
2.0
2.0
2.0
2.0
2.0
2.0
2.0
2.0
2.0
2.0
.0
.0
.0
.0
.0
.0


CHANNEL PESULIS

CASE 1 CFTL BUNOLE - ONE SIXTH SESTION - FULLY ROUGH FLOW - 1/13/81

TIME $=.00000$ SECONDS PRESSURE $=9000.0 \mathrm{KPA}$

$\begin{array}{ccccc}\text { OISTANCE } & \text { OELTA-P } & \text { TEMPERATURE } & \text { DENSITY } & \text { FLOW } \\ \text { (MM) } & \text { (KPA) } & \text { (OEE-C) } & \text { (KG/M3) } & \text { (KG/S) } \\ 10.0 & .0 C 0 & 325.1 & 7.107 & .006101 \\ 186.6 & -1.878 & 326.6 & 7.088 & .006111 \\ 430.0 & -7.435 & 32 E .4 & 7.064 & .006504 \\ 554.8 & -8.636 & 325.3 & 7.051 & .006384 \\ 714.6 & -13.226 & 330.4 & 7.037 & .006676 \\ 805.8 & -14.048 & 331.1 & 7.027 & .006536 \\ 991.2 & -17.464 & 338.5 & 6.942 & .005116 \\ 1072.0 & -25.219 & 358.6 & 6.806 & .006362 \\ 1191.2 & -30.464 & 37 . .7 & 6.583 & .005791 \\ 1272.0 & -38.676 & 387.6 & 6.423 & .006229 \\ 1391.2 & -44.294 & 413.8 & 6.178 & .005728 \\ 1472.0 & -53.041 & 431.5 & 6.021 & .006202 \\ 1591.2 & -59.021 & 459.6 & 5.790 & .005713 \\ 1672.0 & -68.320 & 476.5 & 5.657 & .006195 \\ 1791.2 & -74.627 & 502.6 & 5.465 & .005710 \\ 1872.0 & -84.427 & 516.5 & 5.366 & .006195 \\ 1991.2 & -90.993 & 537.6 & 5.224 & .005714 \\ 2072.0 & -101.189 & 545.8 & 5.161 & .006201 \\ 2179.1 & -106.751 & 554.3 & 5.111 & .005767 \\ 2267.9 & -112.279 & 549.2 & 5.139 & .006425 \\ 2402.8 & -118.281 & 545.1 & 5.161 & .006786 \\ 2544.2 & -120.059 & 543.8 & 5.167 & .006574 \\ 2636.3 & -125.421 & 542.7 & 5.171 & .006864 \\ 2742.3 & -126.718 & 542.3 & 5.172 & .006663 \\ 2860.0 & -132.392 & 541.3 & 5.177 & .006890\end{array}$

DATA FOR CHANNEL

$\begin{array}{ccc}\text { REYNOLOS } & \text { AREA } & \text { FRICTION } \\ \text { NUMBER } & \text { IHM? } & \text { FACTOR } \\ 91555 . & 22.14 & .01926 \\ 91532 . & 22.14 & .01926 \\ 97229 . & 22.14 & .01900 \\ 95325 . & 22.14 & .01909 \\ 99578 . & 22.14 & .01890 \\ 97404 . & 22.14 & .01900 \\ 91935 . & 22.90 & .05160 \\ 94356 . & 22.90 & .11372 \\ 83934 . & 22.90 & .11319 \\ 88796 . & 22.90 & .11253 \\ 79496 . & 22.90 & .11246 \\ 84585 . & 22.90 & .11217 \\ 75859 . & 22.90 & .11247 \\ 80970 . & 22.90 & .111253 \\ 72902 . & 22.90 & .11312 \\ 78128 . & 22.90 & .11345 \\ 70775 . & 22.90 & .111421 \\ 76211 . & 22.90 & .11478 \\ 69253 . & 22.14 & .08927 \\ 77481 . & 22.14 & .02000 \\ 82120 . & 22.14 & .01974 \\ 79632 . & 22.14 & .01987 \\ 83224 . & 22.14 & .01968 \\ 80818 . & 22.14 & .01981 \\ 83648 . & 22.14 & .01965\end{array}$

5

$\begin{array}{ll}\text { STANTON } & \text { FILMH } \\ \text { NUMEER } & \text { KHIM2-C } \\ .00300 & 4.290 \\ .00300 & 4.294 \\ .00296 & 4.505 \\ .00297 & 4.440 \\ .00294 & 4.637 \\ .00295 & 4.521 \\ .00560 & 7.927 \\ .00560 & 7.575 \\ .00574 & 7.750 \\ .00568 & 7.550 \\ .00581 & 7.784 \\ .00575 & 7.612 \\ .00587 & 7.855 \\ .00580 & 7.676 \\ .00591 & 7.906 \\ .00583 & 7.726 \\ .00594 & 7.950 \\ .00585 & 7.755 \\ .0030 .1 & 4.148 \\ .00293 & 4.413 \\ .00290 & 4.782 \\ .0029 ? & 4.493 \\ .00289 & 4.645 \\ .00291 & 4.540 \\ .00289 & 4.747\end{array}$

$R(H \bullet)$

.00

.00

.00
.00

.00

.00

4.37

$4 \cdot 3.7$

2.3 .72

$4.37 \quad 2.0$

$4.37 \quad 2.0$

$4.37 \quad 2.0$

$4.37 \quad 2.0$

$4.37 \quad 2.0$

$4.37 \quad 2.0$

$4.37 \quad 2.0$

$4.37 \quad 2.0$

$\begin{array}{ll}4.37 & 2.0 \\ 4.37 & 2.0\end{array}$

$4.37 \quad 2.0$

$.00 \quad .0$

.00

.00

.00

.00
.0
.0
.0
.0
.0
.0
.0
.0
.0
.0
.0
.0
.0
.0
.0
.0
.0
.0
.0
.0
.0
.0
.0
0 
CHANNEL RESULTS

TIME $=.00000$ SECONDS PRESSURE $=9000.0 \mathrm{KPA}$

$\begin{array}{ccccc}\text { DISTANCE } & \text { DELTA-P } & \text { TEMPERATURE } & \text { OENSITY } & \text { FLOW } \\ \text { (MM) } & \text { (KPAI } & \begin{array}{c}\text { (KEEG-CI } \\ \text { (KG/M3) }\end{array} & \text { (KG/S) } \\ 186.0 & .010 & 325.1 & 7.107 & .010257 \\ 186.6 & -1.879 & 326.6 & 7.087 & .010438 \\ 430.0 & -7.433 & 328.7 & 7.061 & .011511 \\ 554.8 & -8.638 & 329.6 & 7.048 & .011384 \\ 714.6 & -13.222 & 330.8 & 7.032 & .012040 \\ 805.8 & -14.048 & 331.5 & 7.022 & .011841 \\ 991.2 & -17.469 & 338.7 & 6.939 & .011169 \\ 1072.0 & -25.211 & 351.2 & 6.800 & .011479 \\ 1191.2 & -30.471 & 372.3 & 6.577 & .010480 \\ 1272.0 & -38.668 & 389.8 & 6.403 & .011199 \\ 1391.2 & -44.302 & 416.3 & 6.156 & .010324 \\ 1472.0 & -53.032 & 436.2 & 5.982 & .011109 \\ 1591.2 & -59.030 & 464.6 & 5.751 & .010265 \\ 1672.0 & -68.379 & 484.1 & 5.601 & .011067 \\ 1791.2 & -74.636 & 510.5 & 5.411 & .010236 \\ 1872.0 & -84.415 & 526.9 & 5.297 & .011047 \\ 1991.2 & -91.002 & 548.1 & 5.158 & .010227 \\ 2972.0 & -101.176 & 559.3 & 5.085 & .011044 \\ 2179.1 & -106.762 & 566.9 & 5.035 & .010363 \\ 2267.9 & -112.270 & 564.1 & 5.048 & .011460 \\ 2402.8 & -118.274 & 561.3 & 5.062 & .012057 \\ 2544.2 & -120.064 & 559.9 & 5.069 & .011743 \\ 2636.3 & -125.409 & 557.9 & 5.078 & .012289 \\ 2742.3 & -126.721 & 557.0 & 5.082 & .011998 \\ 2860.0 & -132.390 & 555.1 & 5.091 & .012404 \\ & & & & \end{array}$

DATA FOR CHANNEL

$\begin{array}{lll}\text { REYNOLOS } & \text { AREA } & \text { FRICTION } \\ \text { NUMBER } & \text { IHH2) } & \text { FACTOR } \\ 102525 . & 37.23 & .01878 \\ 104149 . & 37.23 & .01872 \\ 114591 . & 37.23 & .01833 \\ 113195 . & 37.23 & .01838 \\ 119563 . & 37.23 & .01816 \\ 117496 . & 37.23 & .01823 \\ 110800 . & 38.26 & .04912 \\ 113333 . & 38.26 & .10807 \\ 101127 . & 38.26 & .10759 \\ 106097 . & 38.26 & .10697 \\ 95209 . & 38.26 & .10691 \\ 110463 . & 38.26 & .10664 \\ 90361 . & 38.26 & .10693 \\ 95691 . & 38.26 & .10699 \\ 86453 . & 38.26 & .10753 \\ 91974 . & 38.26 & .10785 \\ 83635 . & 39.26 & .10854 \\ 89478 . & 38.26 & .10907 \\ 82034 . & 37.23 & .08492 \\ 90924 . & 37.23 & .01929 \\ 95885 . & 37.23 & .01906 \\ 93505 . & 37.23 & .01917 \\ 98001 . & 37.23 & .01897 \\ 95759 . & 37.23 & .01907 \\ 99151 . & 37.23 & .01892\end{array}$

$\begin{array}{ll}\text { STANION } & \text { FILMH } \\ \text { NUMBER } & \text { KH/M2-C } \\ .00295 & 4.221 \\ .00294 & 4.278 \\ .00288 & 4.613 \\ .00288 & 4.573 \\ .00285 & 4.845 \\ .00286 & 4.713 \\ .00536 & 8.199 \\ .00537 & 7.823 \\ .00550 & 8.017 \\ .00545 & 7.784 \\ .00557 & 8.030 \\ .00552 & 7.834 \\ .00563 & 8.087 \\ .00557 & 7.893 \\ .00568 & 8.131 \\ .00561 & 7.938 \\ .00571 & 8.170 \\ .00563 & 7.965 \\ .00291 & 4.311 \\ .00284 & 4.542 \\ .00281 & 4.931 \\ .00283 & 4.629 \\ .00280 & 4.794 \\ .00282 & 4.707 \\ .00279 & 4.949\end{array}$

H+

$R(H+)$

AR

$.0 \quad .00 \quad .0$

$\begin{array}{lll}.0 & .00 & .0\end{array}$

$.0 \quad .00 \quad .0$

$.0 \quad .00 \quad 00$

$\begin{array}{lll}128.8 & 4.37 & 2.0\end{array}$

$111.1 \quad 4.37 \quad 2.0$

$96.7 \quad 4.37 \quad 2.0$

$98.1 \quad 4.37 .2 .0$

$87.7 \quad 4.37 \quad 2.0$

$91.3 \quad 4.37 \quad 2.0$

$83.3 \quad 4.37$

$88.6 \quad 4.37 \quad 2.0$

$82.4 \quad 4.37 \quad 2.0$

$89.1 \quad 4.37 \quad 2.0$

$84.1 \quad 4.37 \quad 2.0$

$92.5 \quad 4.37 \quad 2.0$

$85.4 \quad 4.37 \quad 2.0$

$.0 \quad .00 \quad .0$

$.00 .00 \quad .0$

.00

.00

.00

.00 
CHANNEL QESULTS

CASE 1 CFTL BUNOLE - ONE SIXTH SECTION - FULLY ROUGH FLOH - 1/13/81

DATE 27 MAY 81 TIME $11: 21: 28$

TIME $=.00000$ SECONDS PRESSURE $=9000.0 \mathrm{KPA}$

DISTANCE DELTA-P TEMPERATURE DENSITY

\begin{tabular}{|c|c|c|c|c|}
\hline $\begin{array}{l}\text { STANCE } \\
\text { (MM) }\end{array}$ & $\begin{array}{l}\text { DELTA-P } \\
\text { (KPA) }\end{array}$ & $\begin{array}{c}T \equiv \text { MPERATURE } \\
\text { (DEG }-\mathrm{CI}\end{array}$ & $\begin{array}{l}\text { OENSITY } \\
\text { (KG/MSI) }\end{array}$ & $\begin{array}{l}F L O H \\
(K G / S)\end{array}$ \\
\hline $\begin{array}{r}.0 \\
186.6\end{array}$ & $\begin{array}{r}.000 \\
-1.879\end{array}$ & $\begin{array}{l}325.1 \\
326.6\end{array}$ & $\begin{array}{l}7.107 \\
7.087\end{array}$ & $\begin{array}{l}.005121 \\
.005216\end{array}$ \\
\hline 430.0 & -7.431 & 328.7 & 7.061 & .005772 \\
\hline 554.8 & -8.640 & 329.7 & 7.047 & .005715 \\
\hline 714.6 & -13.219 & 330.9 & 7.031 & .006051 \\
\hline 805.8 & -14.047 & 331.6 & 7.021 & .005956 \\
\hline 991.2 & -17.473 & 338.8 & 6.938 & .005575 \\
\hline 372.0 & -25.203 & 351.2 & 6.799 & .005767 \\
\hline 191.2 & -30.482 & 372.3 & 6.577 & .005271 \\
\hline 272.0 & -33.656 & 390.0 & 6.400 & .005619 \\
\hline 391.2 & -44.315 & 416.7 & 6.153 & .005188 \\
\hline 1472.0 & -53.019 & 437.1 & 5.975 & .005569 \\
\hline 1591.2 & -59.043 & 465.8 & 5.743 & .0051 .55 \\
\hline 672.0 & -68.296 & 486.0 & 5.587 & .005544 \\
\hline 1791.2 & -74.650 & 512.7 & 5.396 & .005137 \\
\hline 1872.0 & -84.401 & 530.1 & 5.277 & .005530 \\
\hline 991.2 & -91.017 & 551.6 & 5.136 & .005130 \\
\hline 2072.0 & $\begin{array}{l}-101.1 \in 2 \\
-106.779\end{array}$ & 563.9 & 5.057 & - 005525 \\
\hline 267.9 & $\begin{array}{r}-106.779 \\
-112.262\end{array}$ & $\begin{array}{l}571.8 \\
570.2\end{array}$ & $\begin{array}{l}5.007 \\
5.013\end{array}$ & $\begin{array}{r}.005195 \\
.005733\end{array}$ \\
\hline 402.8 & -118.270 & 568.4 & 5.020 & .006032 \\
\hline 2544.2 & -120.071 & 567.4 & 5.024 & .005885 \\
\hline 2636.3 & -125.403 & 566.0 & 5.030 & .006150 \\
\hline 742.3 & -126.725 & 565.2 & 33 & .006015 \\
\hline 860.0 & -132.390 & 563.7 & 5.040 & .006215 \\
\hline
\end{tabular}

OATA FOR CHANNEL 6

$\begin{array}{lll}\text { PEYNOLOS } & \text { AREA } & \text { FRICTION } \\ \text { NUMBER } & \text { IMM? } & \text { FACTOR } \\ 102448 . & 18.58 & .01878 \\ 104178 . & 18.58 & .01872 \\ 11503 . & 18.58 & .01831 \\ 113751 . & 18.58 & .01836 \\ 120260 . & 18.58 & .01814 \\ 118291 . & 18.58 & .01820 \\ 111704 . & 19.10 & .04910 \\ 113970 . & 19.10 & .10808 \\ 101806 . & 19.10 & .10762 \\ 106527 . & 19.10 & .10699 \\ 95736 . & 19.10 & .10694 \\ 100730 . & 19.10 & .10667 \\ 90736 . & 19.10 & .10696 \\ 95790 . & 19.10 & .10702 \\ 86679 . & 19.10 & .10757 \\ 91911 . & 19.10 & .10788 \\ 83721 . & 19.10 & .10857 \\ 89260 . & 19.10 & .10910 \\ 81989 . & 18.58 & .08494 \\ 90593 . & 18.58 & .01931 \\ 95460 . & 18.58 & .01908 \\ 93208 . & 18.58 & .01918 \\ 97513 . & 18.58 & .01899 \\ 95436 . & 18.58 & .01908 \\ 98736 . & 18.58 & .01894\end{array}$

$\begin{array}{ll}\text { STANTON } & \text { FILMH } \\ \text { NUMESR } & \text { KW/M2-C } \\ .00295 & 4.221 \\ .00294 & 4.231 \\ .00287 & 4.628 \\ .00288 & 4.593 \\ .00284 & 4.870 \\ .00285 & 4.741 \\ .00535 & 8.254 \\ .00536 & 7.861 \\ .00549 & 8.062 \\ .00545 & 7.814 \\ .00557 & 8.071 \\ .00551 & 7.861 \\ .00563 & 8.126 \\ .00557 & 7.917 \\ .00568 & 8.167 \\ .00561 & 7.961 \\ .00571 & 8.205 \\ .00563 & 7.986 \\ .00291 & 4.324 \\ .00284 & 4.548 \\ .00281 & 4.937 \\ .00283 & 4.641 \\ .00280 & 4.802 \\ .00281 & 4.720 \\ .00279 & 4.961\end{array}$

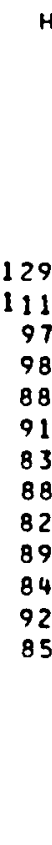

$H+$
.0
.0
.0
.0
.0
.0
29.9
11.8
97.4
98.6
88.4
91.8
83.8
88.8
82.7
89.2
84.3
92.4
85.5
.0
.0
.0
.0
.0
.0

$R(H \bullet)$ 
CHANNEL RESULTS

CASE I CFTL BUNOLE - ONE SIXTH SECTION - FULLY ROUGH FLOH - 1/13/81

TIME $=.00000$ SECONOS PRESSURE $=9000.0 \mathrm{KPA}$

DISTANCE DELTA-P TEMPERATURE DENSITY

\begin{tabular}{|c|c|c|c|c|}
\hline $\begin{array}{l}\text { (STANCE } \\
\text { (MM) }\end{array}$ & $\begin{array}{c}\text { DELTA-P } \\
\text { (KPA) }\end{array}$ & $\begin{array}{l}\text { TE MPERATURE } \\
\text { (DEG -C) }\end{array}$ & $\begin{array}{l}\text { DENSITY } \\
\text { IKG/MSI }\end{array}$ & $\begin{array}{l}F L O H \\
|K G / S|\end{array}$ \\
\hline .0 & .000 & 325.1 & 7.107 & .005121 \\
\hline 186.6 & -1.877 & 326.6 & 7.088 & .005211 \\
\hline 430.0 & -7.436 & 328.5 & 7.063 & .005685 \\
\hline 554.8 & -8.635 & 329.4 & 7.050 & .005617 \\
\hline 714.6 & -13.229 & 330.6 & 7.034 & \\
\hline 805.8 & $-14.0 \leq 0$ & 331.2 & 7.025 & .005818 \\
\hline 991.2 & -17.458 & 338.5 & 6.941 & .005482 \\
\hline 1072.0 & $-25.2 \geq 2$ & 350.6 & 6.805 & .005698 \\
\hline 191.2 & -30.438 & 371.5 & 6.585 & .005227 \\
\hline$? 72.0$ & $-38.7 C 0$ & 387.5 & 6.424 & .005590 \\
\hline 391.2 & -44.267 & 413.8 & 6.179 & .005168 \\
\hline 1472.0 & -53.067 & 431.6 & 6.020 & .005563 \\
\hline 591.2 & -58.993 & 460.1 & 5.786 & .005149 \\
\hline 672.0 & -68.347 & 477.8 & 5.648 & .005551 \\
\hline 1791.2 & -74.597 & 504.6 & 5.451 & .005141 \\
\hline 1872.0 & -84.454 & 519.9 & 5.343 & .005544 \\
\hline 1991.2 & -90.962 & 541.7 & 5.198 & .005140 \\
\hline 072.0 & -101.217 & 552.9 & 5.124 & .005543 \\
\hline 2179.1 & -106.715 & 560.9 & 5.071 & .005206 \\
\hline 2267.9 & -112.295 & 559.4 & 5.077 & .005748 \\
\hline 402.8 & -118.282 & 556.8 & 5.089 & .006072 \\
\hline 44.2 & -120.048 & 554.5 & 5.101 & .00592 \\
\hline 2636.3 & -125.426 & 553.3 & 5.106 & .006196 \\
\hline 2742.3 & 26.76 .9 & 551.7 & 5.114 & .00605 \\
\hline 16 & 32.390 & 550.0 & 5.122 & .00627 \\
\hline
\end{tabular}

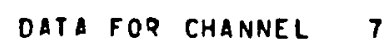

$\begin{array}{lll}\text { REYNOLOS } & \text { AREA } & \text { FRICIION } \\ \text { NUMEER } & \text { IMMEI } & \text { FACTOR } \\ 102448 . & 18.58 & .01878 \\ 104070 . & 18.58 & .01872 \\ 113291 . & 18.58 & .01837 \\ 111813 . & 18.58 & .01843 \\ 117627 . & 18.58 & .01822 \\ 115587 . & 18.58 & .01829 \\ 109860 . & 19.10 & .04914 \\ 112666 . & 19.10 & .10805 \\ 101042 . & 19.10 & .10758 \\ 106262 . & 19.10 & .10695 \\ 95643 . & 19.10 & .10690 \\ 101143 . & 19.10 & .10662 \\ 91110 . & 19.10 & .10691 \\ 96622 . & 19.10 & .10696 \\ 87357 . & 19.10 & .10751 \\ 92952 . & 19.10 & .10782 \\ 84583 . & 19.10 & .10853 \\ 90372 . & 19.10 & .10906 \\ 82908 . & 18.58 & .08490 \\ 91649 . & 18.58 & .01926 \\ 97024 . & 18.58 & .01901 \\ 94860 . & 18.58 & .01911 \\ 99284 . & 18.58 & .01892 \\ 97189 . & 18.58 & .01901 \\ 100756 . & 18.58 & .01885\end{array}$

DATE 27 MAY B1

TIME $11: 21: 28$

$\begin{array}{ccccc}\text { STANTON } & \text { FILMH } & H+ & R(H+1 & A R \\ \text { NUMBER } & \text { KHIM2-C } & & & \\ .00295 & 4.221 & .0 & .00 & .0 \\ .00294 & 4.278 & .0 & .00 & .0 \\ .00288 & 4.574 & .0 & .00 & .0 \\ .00289 & 4.532 & .0 & .00 & .0 \\ .00286 & 4.787 & .0 & .00 & .0 \\ .00287 & 4.656 & .0 & .00 & .0 \\ .00537 & 8.152 & 127.6 & 4.37 & 2.0 \\ .00537 & 7.791 & 110.3 & 4.37 & 2.0 \\ .00550 & 8.011 & 96.5 & 4.37 & 2.0 \\ .00545 & 7.781 & 98.1 & 4.37 & 2.0 \\ .00557 & 8.043 & 98.1 & 4.37 & 2.0 \\ .00551 & 7.845 & 91.7 & 4.37 & 2.0 \\ .00562 & 8.110 & 84.0 & 4.37 & 2.0 \\ .00556 & 7.910 & 89.3 & 4.37 & 2.0 \\ .00567 & 8.159 & 83.2 & 4.37 & 2.0 \\ .00559 & 7.960 & 90.0 & 4.37 & 2.0 \\ .00570 & 8.202 & 85.0 & 4.37 & 2.0 \\ .00562 & 7.989 & 93.4 & 4.37 & 2.0 \\ .00291 & 4.334 & 86.3 & 4.37 & 2.0 \\ .00284 & 4.560 & .0 & .00 & .0 \\ .00281 & 4.965 & .0 & .00 & .0 \\ .00282 & 4.669 & .0 & .00 & .0 \\ .00279 & 4.832 & .0 & .00 & .0 \\ .00281 & 4.749 & .0 & .00 & .0 \\ .00279 & 4.997 & .0 & .00 & .0\end{array}$


CHANNEL PESULTS

CASE

1
TIME $=.00000$ SECONOS PRESSURE $=9000.0 \mathrm{KPA}$

\begin{tabular}{|c|c|c|c|c|}
\hline $\begin{array}{l}\text { OISTANCE } \\
\text { (MM) }\end{array}$ & $\begin{array}{l}\text { DELTA -P } \\
\text { (KPA) }\end{array}$ & $\begin{array}{l}\text { TEMPERATURE } \\
\text { (OEG-C) }\end{array}$ & $\begin{array}{l}\text { DENSITY } \\
\text { (KE/MBI }\end{array}$ & $\begin{array}{l}F L O W \\
(K G / S)\end{array}$ \\
\hline .0 & .060 & 325.1 & 7.107 & $\because 010257$ \\
\hline 186.6 & -1.878 & 326.6 & 7.087 & .010448 \\
\hline 430.0 & -7.435 & 328.6 & 7.061 & .011543 \\
\hline 554.8 & -8.637 & 329.6 & 7.048 & .011418 \\
\hline 714.6 & -13.226 & 330.8 & 7.032 & .012085 \\
\hline 805.8 & -14.049 & 331.4 & 7.022 & .011886 \\
\hline 991.2 & -17.463 & 338.7 & 6.940 & .011126 \\
\hline 1072.0 & -25.223 & 351.0 & 6.801 & .011542 \\
\hline 1191.2 & -30.452 & 372.1 & 6.579 & .010535 \\
\hline 1272.0 & -38.686 & 389.3 & 6.407 & .011274 \\
\hline 1391.2 & $-44.2 E 2$ & $4 i 5.7$ & 6.162 & .010385 \\
\hline 1472.0 & -53.052 & 435.0 & 5.992 & .011192 \\
\hline 1591.2 & -59.009 & 463.1 & 5.763 & .010332 \\
\hline 1672.0 & -68.331 & 481.8 & 5.618 & .011158 \\
\hline 1791.2 & -74.613 & 508.0 & 5.428 & .010310 \\
\hline $1872 . n$ & -84.437 & 523.4 & 5.320 & .011147 \\
\hline 1991.2 & -90.979 & 544.4 & 5.181 & .010309 \\
\hline 2072.0 & -101.200 & $554 \cdot 6$ & 5.113 & .011154 \\
\hline 2179.1 & -106.734 & 562.1 & 5.064 & .010451 \\
\hline 2267.9 & $-112.2 \varepsilon 3$ & $558 \cdot 1$ & 5.084 & .011588 \\
\hline 2402.8 & -118.279 & 553.7 & 5.108 & .012179 \\
\hline 2544.2 & -120.056 & 551.6 & 5.119 & .011852 \\
\hline 2636.3 & -125.418 & 549.7 & 5.128 & .012407 \\
\hline 2742.3 & -126.715 & $548 \cdot 7$ & 5.133 & .012103 \\
\hline 2860.0 & -132.390 & 546.6 & 5.143 & .012512 \\
\hline
\end{tabular}

DATA FOR CHANNEL 8

$\begin{array}{ccc}\text { REYNOLOS } & \text { AREA } & \text { FRICIION } \\ \text { NUMBER } & \text { IMM2 } & \text { FACTOR } \\ 102525 . & 37.23 & .01878 \\ 104253 . & 37.23 & .01871 \\ 114909 . & 37.23 & .01832 \\ 113541 . & 37.23 & .01837 \\ 120010 . & 37.23 & .01815 \\ 117945 . & 37.23 & .01821 \\ 111377 . & 38.26 & .04911 \\ 113974 . & 38.26 & .10808 \\ 101676 . & 38.26 & .10761 \\ 106.861 . & 38.26 & .10699 \\ 95832 . & 38.26 & .10692 \\ 101334 . & 38.26 & .10666 \\ 91073 . & 38.26 & .10694 \\ 96681 . & 38.26 & .10700 \\ 87265 . & 38.26 & .10754 \\ 93087 . & 38.26 & .10786 \\ 84565 . & 38.26 & .10855 \\ 90720 . & 38.26 & .10908 \\ 83062 . & 37.23 & .08491 \\ 92395 . & 37.23 & .01922 \\ 97467 . & 37.23 & .01899 \\ 95015 . & 37.23 & .01910 \\ 99622 . & 37.23 & .01890 \\ 97261 . & 37.23 & .01900 \\ 100724 . & 37.23 & .01886\end{array}$

DATE 27 MAY 8

TIME

$11: 21: 28$

$\begin{array}{ll}\text { STANTON } & \text { FILMH } \\ \text { NUMBER } & \text { KHIMZ - } \\ .00295 & 4.221 \\ .00294 & 4.231 \\ .00287 & 4.622 \\ .00288 & 4.583 \\ .00284 & 4.859 \\ .00285 & 4.727 \\ .00535 & 8.230 \\ .00536 & 7.854 \\ .00549 & 8.047 \\ .00544 & 7.821 \\ .00557 & 8.064 \\ .00551 & 7.875 \\ .00562 & 8.124 \\ .00556 & 7.936 \\ .00567 & 8.170 \\ .00559 & 7.986 \\ .00570 & 8.213 \\ .00561 & 8.016 \\ .00291 & 4.340 \\ .00284 & 4.582 \\ .00281 & 4.970 \\ .00282 & 4.664 \\ .00279 & 4.832 \\ .00281 & 4.740 \\ .00279 & 4.983\end{array}$

\begin{tabular}{rrr}
$H+$ & $R(H+)$ & \multicolumn{1}{l}{$A R$} \\
.0 & .00 & .0 \\
.0 & .00 & .0 \\
.0 & .00 & .0 \\
.0 & .00 & .0 \\
.0 & .00 & .0 \\
.0 & .00 & .0 \\
129.5 & 4.37 & 2.0 \\
111.8 & 4.37 & 2.0 \\
97.3 & 4.37 & 2.0 \\
98.9 & 4.37 & 2.0 \\
88.4 & 4.37 & 2.0 \\
92.1 & 4.37 & 2.0 \\
84.1 & 4.37 & 2.0 \\
89.5 & 4.37 & 2.0 \\
83.2 & 4.37 & 2.0 \\
90.2 & 4.37 & 2.0 \\
85.0 & 4.37 & 2.0 \\
93.8 & 4.37 & 2.0 \\
86.5 & 4.37 & 2.0 \\
.0 & .00 & .0 \\
.0 & .00 & .0 \\
.0 & .00 & .0 \\
.0 & .00 & .0 \\
.0 & .00 & .0 \\
.0 & .00 & .0
\end{tabular}


CHANNEL RESULTS

TIME $=.00000$ SECONDS PRESSURE $=9000.0 \mathrm{KPA}$

$\begin{array}{ccccc}\text { DISTANCE } & \text { OELTA-P } & \text { TEMPERATURE } & \text { DENSITY } & \text { FLON } \\ \text { IMMI } & \text { (KPAI } & \begin{array}{c}\text { IOEG-CI } \\ \text { IKGIMSI }\end{array} & \text { IKG/SI } \\ .0 & .000 & 325.1 & 7.107 & .010257 \\ 186.6 & -1.877 & 326.6 & 7.088 & .010438 \\ 430.0 & -7.438 & 328.5 & 7.063 & .011426 \\ 554.8 & -8.624 & 329.4 & 7.049 & .011283 \\ 714.6 & -13.231 & 330.6 & 7.034 & .011997 \\ 805.8 & -14.051 & 331.2 & 7.025 & .011691 \\ 991.2 & -17.455 & 338.5 & 6.941 & .010984 \\ 1072.0 & -25.238 & 350.8 & 6.804 & .011450 \\ 1191.2 & -30.431 & 371.8 & 6.582 & .010475 \\ 1272.0 & -38.777 & 387.9 & 6.421 & .011233 \\ 1391.2 & -44.258 & 413.9 & 6.177 & .010354 \\ 1472.0 & -53.076 & 431.2 & 6.024 & .011178 \\ 1591.2 & -58.982 & 458.8 & 5.796 & .010321 \\ 1672.0 & -68.357 & 474.9 & 5.669 & .011166 \\ 1791.2 & -74.584 & 500.3 & 5.481 & .010319 \\ 1872.0 & -84.465 & 513.2 & 5.388 & .011173 \\ 1991.2 & -90.948 & 533.7 & 5.249 & .010333 \\ 2072.0 & -101.229 & 541.9 & 5.192 & .011190 \\ 2179.1 & -106.698 & 549.2 & 5.142 & .010475 \\ 2267.9 & -112.295 & 544.3 & 5.169 & .011539 \\ 2402.8 & -118.289 & 540.6 & 5.189 & .012010 \\ 2544.2 & -120.042 & 539.1 & 5.196 & .011625 \\ 2636.3 & -125.430 & 537.8 & 5.202 & .012153 \\ 2742.3 & -126.706 & 537.1 & 5.205 & .011808 \\ 2860.0 & -132.390 & 536.3 & 5.208 & .012182\end{array}$

DATE 27 MAY 81

IIME $11: 21: 28$
OATA FOR CHANNEL

$\begin{array}{ccc}\text { REYNOLDS } & \text { AREA } & \text { FRICIION } \\ \text { NUMAER } & \text { IMM? } & \text { FACTOR } \\ 102525 . & 37.23 & .01878 \\ 104151 . & 37.23 & .01872 \\ 113757 . & 37.23 & .01836 \\ 112221 . & 37.23 & .01841 \\ 118167 . & 37.23 & .01821 \\ 116042 . & 37.23 & .01828 \\ 169969 . & 38.26 & .04914 \\ 113093 . & 38.26 & .10806 \\ 101130 . & 38.26 & .10758 \\ 106641 . & 38.26 & .10696 \\ 95715 . & 38.26 & .10690 \\ 101592 . & 38.26 & .10662 \\ 91353 . & 38.26 & .10690 \\ 97368 . & 38.26 & .10695 \\ 87935 . & 38.26 & .10749 \\ 94137 . & 38.26 & .10780 \\ 85537 . & 38.26 & .10850 \\ 91989 . & 38.26 & .10903 \\ 84144 . & 37.23 & .08486 \\ 93072 . & 37.23 & .01919 \\ 97177 . & 37.23 & .01901 \\ 94181 . & 37.23 & .01914 \\ 98599 . & 37.23 & .01895 \\ 95821 . & 37.23 & .01907 \\ 98925 . & 37.23 & .01893\end{array}$

STANTON
NUMBER
.00295
.00294
.00288
.00289
.00285
.00286
.00537
.00537
.00550
.00544
.00557
.00550
.00562
.00555
.00566
.00558
.00568
.00560
.00291
.00284
.00282
.00284
.00281
.00283
.00281

FILMH
$K H / M 2-C$
4.221
4.278
4.586
4.542
4.801
4.667
8.152
7.808
8.013
7.798
8.043
7.861
8.110
7.929
8.163
7.983
8.210
8.016
4.350
4.570
4.920
4.597
4.757
4.653
4.884

$A P$
$R I H+1$

H4

0

.0

0

.0
.0
.0

127.0

96.6

88.1

92.2

84.1

89.9

83.6

91.0

05.

$94.9 \quad 4.37$

87.54 .37

4.37
.00

.00

.00

.00

.00
.00
.0
.0
.0
.0
.0
.0
2.0
2.0
2.0
2.0
2.0
2.0
2.0
2.0
2.0
2.0
2.0
2.0
2.0
.0
.0
.0
.0
.00
.0 
CHANNEL RESULTS

CASE I T CFTL BUNOLE - ONE SIXTH SEETION - FULLY FOUGH FLOH - $1 / 13 / 81$

TIME $=.00000$ SECONOS PRESSURE $=9000.0 \mathrm{KPA}$

\begin{tabular}{|c|c|c|c|c|c|}
\hline & $\begin{array}{l}\text { DISTANCE } \\
\text { (MM) }\end{array}$ & $\begin{array}{l}\text { OELTA-P } \\
\text { (KPA) }\end{array}$ & $\begin{array}{l}\text { TEMPERATURE } \\
\text { (OEE - CI }\end{array}$ & $\begin{array}{l}\text { DENSITY } \\
\text { (KG/MZI }\end{array}$ & $\begin{array}{l}\text { FLOH } \\
\text { |KG/S }\end{array}$ \\
\hline & $\begin{array}{r}.0 \\
.86 .6\end{array}$ & $\begin{array}{r}000 \\
-1076\end{array}$ & 325.1 & 7.107 & .010257 \\
\hline & 186.6 & -1.876 & 326.6 & 7.088 & .010426 \\
\hline & 430.0 & -7.438 & $32 E \cdot 5$ & 7.063 & .011375 \\
\hline & 554.8 & -8.633 & 320.4 & 7.050 & .011204 \\
\hline & 714.6 & -13.232 & 330.5 & 7.035 & .011804 \\
\hline & 805.8 & -14.051 & $33: .2$ & 7.026 & .011570 \\
\hline & 991.2 & -17.454 & 338.5 & 6.942 & .010826 \\
\hline & 1072.0 & -25.240 & 350.8 & 6.803 & .011352 \\
\hline & 1191.2 & -30.429 & $37: .8$ & 6.582 & .010355 \\
\hline & 1272.0 & -38.708. & $38^{\circ} .8$ & 6.421 & .011167 \\
\hline & 1391.2 & -44.256 & 413.4 & 6.182 & .010259 \\
\hline & 1472.0 & -53.078 & 431.3 & 6.031 & .011128 \\
\hline & 1591.2 & -58.979 & 457.1 & $5.800^{\circ}$ & .010237 \\
\hline & 1672.0 & -68.359 & $47 ? .6$ & 5.686 & .011128 \\
\hline & 1791.2 & -74.581 & 497.1 & 5.504 & .010245 \\
\hline & 1872.0 & -84.468 & 50.7 .1 & 5.416 & .011148 \\
\hline & 1991.2 & -90.943 & 528.6 & 5.281 & .010269 \\
\hline & 2072.0 & -101.232 & 535.9 & 5.230 & .011177 \\
\hline & 2179.1 & -106.693 & 542.8 & 5.182 & .010417 \\
\hline & 2267.9 & -112.294 & 536.8 & 5.216 & .011538 \\
\hline & 2402.8 & -118.291 & 552.4 & 5.241 & .011949 \\
\hline & 2544.2 & -120.041 & 531.0 & 5.247 & .011432 \\
\hline & 2636.3 & -125.431 & 529.6 & 5.255 & .012012 \\
\hline & 2742.3 & -126.706 & 529.3 & 5.254 & .011586 \\
\hline & 2860.0 & -132.390 & 528.5 & 5.257 & .011981 \\
\hline
\end{tabular}

DATA FOR CHANNEL 10

$\begin{array}{ll}\text { PEYNOLDS } & \text { AREA } \\ \text { NUMEER } & \text { IMM21 } \\ 102525 . & 37.23 \\ 104034 . & 37.23 \\ 113257 . & 37.23 \\ 111435 . & 37.23 \\ 117258 . & 37.23 \\ 114844 . & 37.23 \\ 108401 . & 38.26 \\ 112120 . & 38.26 \\ 99974 . & 38.26 \\ 106014 . & 38.26 \\ 94882 . & 38.26 \\ 101216 . & 38.26 \\ 90753 . & 38.26 \\ 97238 . & 38.26 \\ 8755 . & 38.26 \\ 94265 . & 38.26 \\ 85371 . & 38.26 \\ 92351 . & 38.26 \\ 84127 . & 37.23 \\ 93654 . & 37.23 \\ 97360 . & 37.23 \\ 93251 . & 37.23 \\ 98109 . & 37.23 \\ 94645 . & 37.23 \\ 97937 . & 37.23\end{array}$

DATE 27 MAY 1

TIME 11:21:28

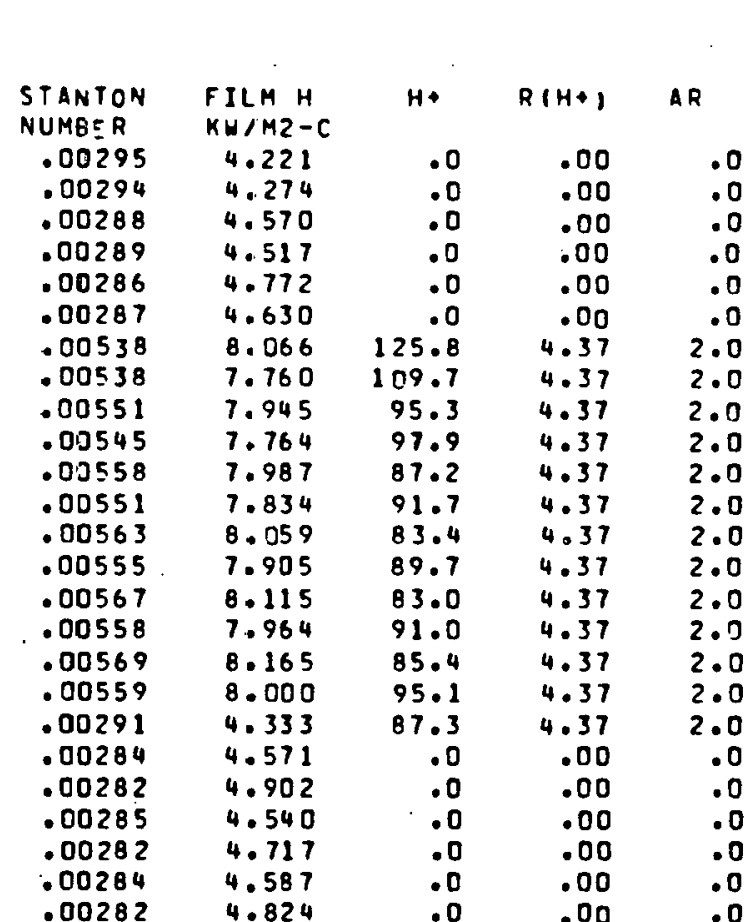


CHANNEL RESULIS

CASE 1 CFTL BUNDLE - ONE SIXTH SECTION - FULLY ROUGH FLON - 1/13/BI

TIME $=.00000$ SECONOS PRESSURE $=9000.0 \mathrm{KPA}$

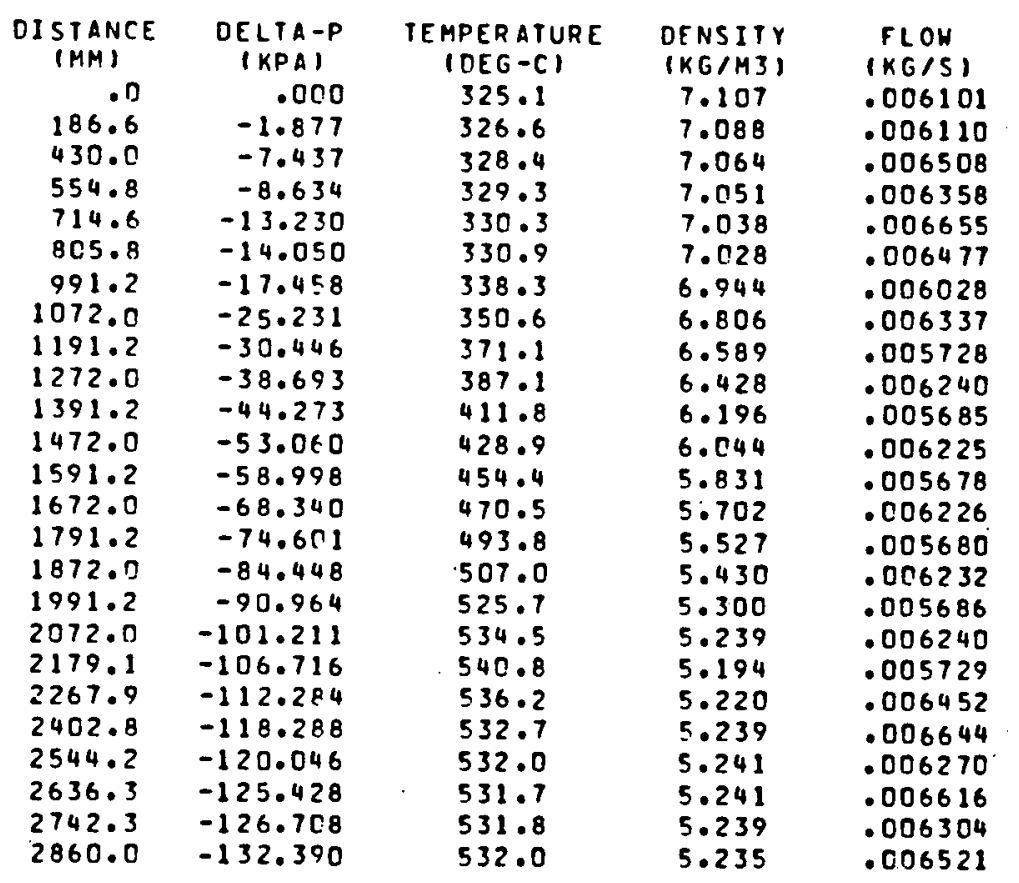

ME $\quad 11: 21: 28$
DATA FOR CHANNEL 11

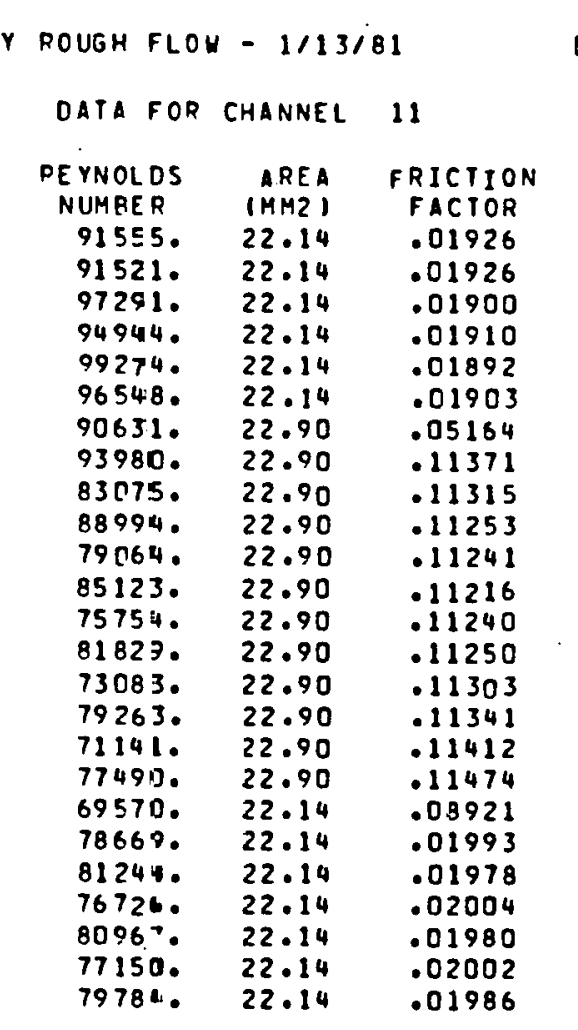

STANTON
NUMBER
.00300
.00300
.00296
.00297
.00294
.00296
.00561
.00561
.00575
.00568
.00582
.00574
.00587
.00578
.00591
.00581
.00594
.00583
.00301
.00293
.00291
.00295
.00291
.00295
.00292

$F I L M H$
$K H / M 2-C$
4.290
4.294
4.507
4.427
4.626
4.490
7.842
7.552
7.687
7.558
7.738
7.628
7.810
7.695
7.861
7.746
7.903
7.772
4.129
4.430
4.707
4.335
4.517
4.353
4.552

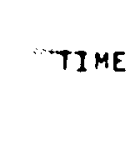
DATE 27 MAY 81 TIME

DATE 27

${ }_{R} N$

$\begin{array}{lll}532.0 & 5.235 & .006521\end{array}$


CHANNEL RESULTS

CASE I CFTL BUNDLE - ONE SIXTH SECTION - FULLY ROUGH FLON - 1/13/81

TIME $=.00000$ SECONLS PRESSURE $=9000.0 \mathrm{KPA}$

DISTANCE DELTA-P TEMPERATURE DENSITY

\begin{tabular}{|c|c|c|c|c|}
\hline $\begin{array}{l}\text { I STANCE } \\
\text { IMMI I }\end{array}$ & $\begin{array}{l}\text { DELTA-P } \\
\text { (KPA) }\end{array}$ & $\begin{array}{l}\text { TE MPER ATURE } \\
\text { (DEG -C) }\end{array}$ & $\begin{array}{l}\text { DENSITY } \\
\text { (KG/M3) }\end{array}$ & $\begin{array}{l}F L O N \\
(K G / S)\end{array}$ \\
\hline .0 & .000 & 325.1 & 7.107 & .003059 \\
\hline 186.6 & -1.876 & 326.5 & 7.089 & .003060 \\
\hline 430.0 & -7.439 & 32.1 .1 & 7.067 & .003190 \\
\hline $554 \cdot B$ & -8.633 & 327.0 & 7.055 & 03104 \\
\hline 714.5 & -13.233 & 327.9 & 7.042 & .003214 \\
\hline 805.8 & -14.050 & 333.5 & 7.033 & .003124 \\
\hline 991.2 & -17.453 & 337.4 & 6.954 & .002932 \\
\hline $1072: 0$ & -25.239 & 349.2 & 6.820 & .003096 \\
\hline 1191.2 & -30.434 & 367.9 & 6.622 & .002821 \\
\hline 1272.0 & -38.703 & 382.5 & 6.472 & .003074 \\
\hline 1391.2 & -44.260 & 404.3 & 6.263 & .002815 \\
\hline 1472.0 & -53.072 & 420.0 & 6.119 & .003076 \\
\hline 1591.2 & -58.984 & 443.1 & 5.921 & .002817 \\
\hline 1672.0 & -68.354 & 458.0 & 5.798 & $.003 n 81$ \\
\hline 1791.2 & -74.585 & 480.0 & 5.626 & .002819 \\
\hline 1872.0 & $-84.4 \in 2$ & 492.3 & 5.533 & .003083 \\
\hline 1991.2 & -90.946 & 511.3 & 5.396 & .002818 \\
\hline 2072.0 & -101.225 & 519.5 & 5.336 & .003082 \\
\hline 2179.1 & -106.694 & 528.0 & 5.276 & .002821 \\
\hline 2267.9 & -112.287 & $523 \cdot 0$ & 5.305 & .003125 \\
\hline 2402.8 & -118.291 & 522.9 & 5.303 & .003082 \\
\hline 2544.2 & -120.036 & 525.4 & 5.284 & .002861 \\
\hline 2636.3 & -125.433 & $52.5 \cdot 5$ & 5.281 & .003039 \\
\hline 2742.3 & -126.700 & 527.4 & 5.267 & .002849 \\
\hline $2860 \cdot \mathrm{C}$ & -132.389 & 528.0 & 5.261 & .00294 \\
\hline
\end{tabular}

DATA FOR CHANNEL 12

$\begin{array}{ll}\text { REYNOLDS } & \text { AREA } \\ \text { NUMBER } & \text { IAM? } \\ 91794 . & 11.10 \\ 91678 . & 11.10 \\ 95388 . & 11.10 \\ 92732 . & 11.10 \\ 95922 . & 11.10 \\ 92869 . & 11.10 \\ 88257 . & 11.48 \\ 91977 . & 11.48 \\ 82108 . & 11.48 \\ 88096 . & 11.48 \\ 78903 . & 11.48 \\ 84863 . & 11.48 \\ 75987 . & 11.48 \\ 81932 . & 11.48 \\ 73449 . & 11.48 \\ 79455 . & 11.48 \\ 71401 . & 11.48 \\ 77527 . & 11.48 \\ 69283 . & 11.10 \\ 77070 . & 11.10 \\ 76021 . & 11.10 \\ 70404 . & 11.10 \\ 74781 . & 11.10 \\ 69996 . & 11.10 \\ 72193 . & 11.10\end{array}$

$\begin{array}{lcc}\text { FRICTION } & \text { STANTON } & \text { FILMH } \\ \text { FACTOR } & \text { NUMBER } & \text { KH/M2-C } \\ .01925 & .00300 & 4.290 \\ .01925 & .00300 & 4.290 \\ .01908 & .00297 & 4.429 \\ .01921 & .00299 & 4.337 \\ .01906 & .00297 & 4.495 \\ .01920 & .00299 & 4.348 \\ .05170 & .00564 & 7.665 \\ .11361 & .00563 & 7.405 \\ .11305 & .00577 & 7.577 \\ .11242 & .00569 & 7.450 \\ .11228 & .00583 & 7.652 \\ .11202 & .00574 & 7.531 \\ .11226 & .00587 & 7.729 \\ .11234 & .00578 & 7.599 \\ .11288 & .00591 & 7.777 \\ .11326 & .00581 & 7.645 \\ .11400 & .00593 & 7.810 \\ .11462 & .00583 & 7.661 \\ .08914 & .00303 & 4.076 \\ .02002 & .00296 & 4.317 \\ .02008 & .00297 & 4.440 \\ .02044 & .00302 & 4.033 \\ .02016 & .00298 & 4.225 \\ .02047 & .00302 & 4.020 \\ .02032 & .00300 & 4.198\end{array}$

DATE 27 MAY 81 TIME $11: 21: 28$

\begin{tabular}{rrr}
$H+$ & $R I H+1$ & \multicolumn{1}{l}{$A R$} \\
.0 & .00 & .0 \\
.0 & .00 & .0 \\
.0 & .00 & .0 \\
.0 & .00 & .0 \\
.0 & .00 & .0 \\
.0 & .00 & .0 \\
118.2 & 4.37 & 2.0 \\
103.1 & 4.37 & 2.0 \\
89.4 & 4.37 & 2.0 \\
92.9 & 4.37 & 2.0 \\
82.6 & 4.37 & 2.0 \\
87.7 & 4.37 & 2.0 \\
79.5 & 4.37 & 2.0 \\
86.1 & 4.37 & 2.0 \\
79.3 & 4.37 & 2.0 \\
87.5 & 4.37 & 2.0 \\
81.6 & 4.37 & 2.0 \\
91.4 & 4.37 & 2.0 \\
82.1 & 4.37 & 2.0 \\
.0 & .00 & .0 \\
.0 & .00 & .0 \\
.0 & .00 & .0 \\
.0 & .00 & .0 \\
.0 & .00 & .0 \\
.0 & .00 & .0
\end{tabular}


CHANNEL RESULIS

CASE 1 CFTL BUNDLE - ONE SIXTH SECTION - FULLY ROUGH FLON - 1/13/81

TIME $=.00000$ SECONDS FRESSURE $=9000.0 \mathrm{KPA}$

DISTANCE DELTA-P TEMPERATURE DENSITY

186.

186.6
430.0

554.8

714.6

991.2

1072.0

1191.2

1272.0

1391.2

1472.0

1591.2

1672.0

1791.2

1872.0

1991.2

2072.0

2179.1

2267.9

2402.8

2544.2

2636.3
2742.3

2860.0

.000
-1.875

-1.875
-7.441

$-8.631$

$-13.238$

$-14.052$

$-17.446$

$-25.253$

$-30.412$

$-38.726$

$-44.236$

$-58.957$

$-68.381$

$-74.557$

$-84.491$

$-90.917$

$-101.256$

$-106.660$

$-118.299$

$-120.028$

$-120.028$

$-125.442$

-126.696
-132.391

325

325.1

(KG/MB)

FLON

(KG/S)

.001713

.001724

.001730

7.070

7.058

7.045

329.6

330.3

337.3

348.1

367.0

378.3

401.6

413.1

438.9

438.4

448.9

473.3

481.7

502.9

508.3

518.5

$5,3.1$

512.8

512.8

514.7

515.0

516.7
517.5

7.036

6.955

6.832

6.630

6.513

6.288

6.180

5.959

5.869

5.676

5.609

5.453

5.411

5.338

5.371

5.370

5.375

5.355

5.350

5.337

5.330

.001711

001713

.001696

001660

.001713

.001648

001730

001665

001744

.001675

$00 ! 753$

.001684

001760

.001691

.001764

001657

001750

.001716

.001647

.001666

.001613

.001629
DATA FOR CHANNEL 13

$\begin{array}{ccc}\text { REYNOLOS } & \text { AREA } & \text { FRICTION } \\ \text { NUMBER } & \text { IMAR I } & \text { FACTOR } \\ 102823 . & 6.22 & .01877 \\ 103310 . & 6.22 & .01875 \\ 103501 . & 6.22 & .01874 \\ 102297 . & 6.22 & .01879 \\ 102301 . & 6.22 & .01879 \\ 101163 . & 6.22 & .01884 \\ 99914 . & 6.39 & .04940 \\ 101915 . & 6.39 & .10786 \\ 96027 . & 6.39 & .10768 \\ 99602 . & 6.39 & .10676 \\ 93554 . & 6.39 & .10703 \\ 96879 . & 6.39 & .10639 \\ 90796 . & 6.39 & .10701 \\ 94041 . & 6.39 & .10670 \\ 88309 . & 6.39 & .10756 \\ 91555 . & 6.39 & .10756 \\ 86324 . & 6.39 & .10855 \\ 89614 . & 6.39 & .10884 \\ 84017 . & 6.22 & .08479 \\ 87078 . & 6.22 & .01948 \\ 85384 . & 6.22 & .01956 \\ 81827 . & 6.22 & .01975 \\ 82745 . & 6.22 & .01970 \\ 79967 . & 6.22 & .01985 \\ 80709 . & 6.22 & .01981\end{array}$

DATE 27 MAY 81 TIME 11:21:28

DATE 27 MAY 81 TIME 11:21:28

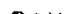

FILM H

H

$R(H+)$

$\begin{array}{llll}.00295 & 4.221 & .0 & .00 \\ .00295 & 4.241 & .0 & .00\end{array}$

$.00295 \quad 4.241$

4.961

4.397

00029

5.138

4.425

8.128

00547

7.498

8.209

7.632

8. 361

.00552

8.482

7.839

8.558

7.902

8.622

7.935

4.374

4.679

5. 419

4.169

4.745

5.040

.0
86.0
98.8
92.2
91.
86.7
84.
85.7
84.
86.8
91.5
86.9
.0
.0
.0

.00
.00

.00

.00

4.37

4.37

$4 \cdot 37$

$4 \cdot 37$

4.37

4.37

4.37

4.37

4.37

4.37

4.37

4.37

4.37

.00
.00
.00

.00

.00

.00

.00 
CHANNEL FESUL TS

CASE

T:ME $=.00000$ SECONOS

PRESSURE $=9000.3 \mathrm{KPA}$

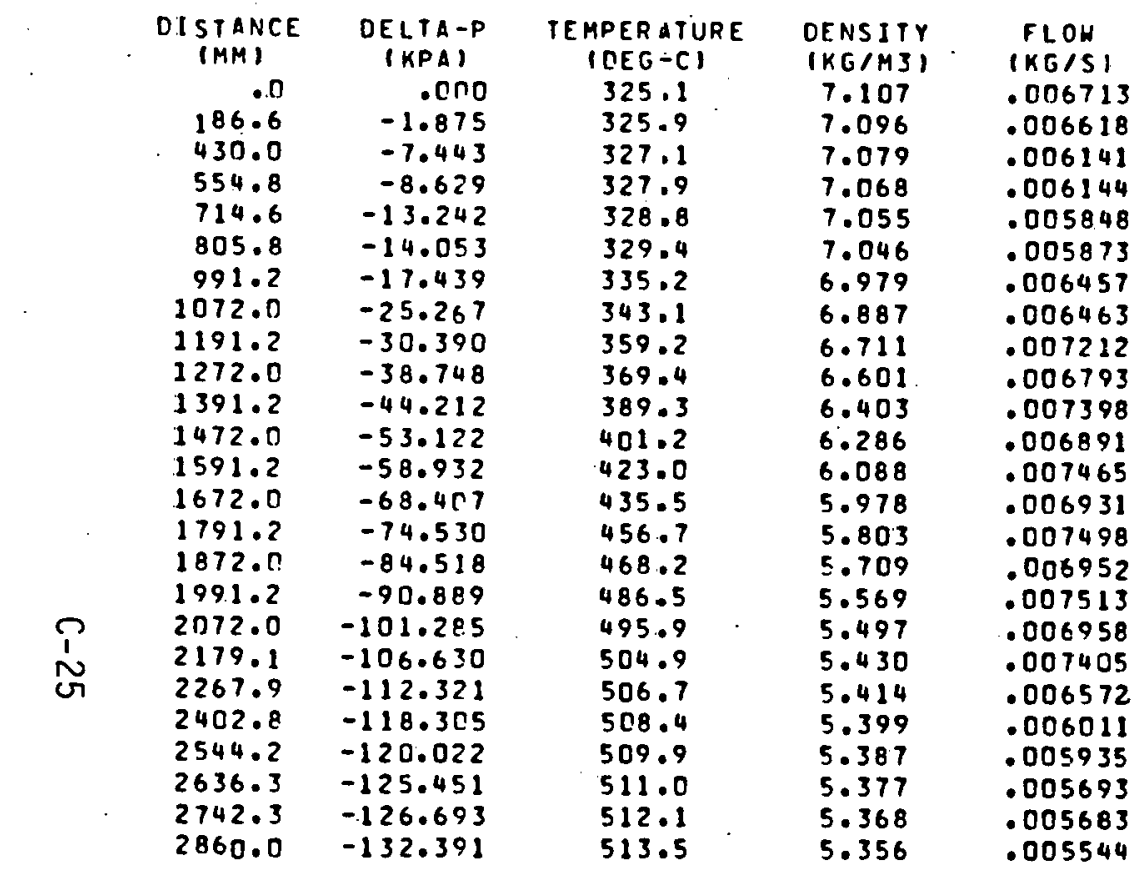

DATA FOR CHANNEL 14

$\begin{array}{cl}\text { REYNOLOS } & \text { AREA } \\ \text { NUMBER } & \text { (MM2) } \\ 81781 . & 24.36 \\ 80549 . & 24.36 \\ 74634 . & 24.36 \\ 74613 . & 24.36 \\ 70944 . & 24.36 \\ 71200 . & 24.36 \\ 79088 . & 24.77 \\ 78469 . & 24.77 \\ 86025 . & 24.77 \\ 80137 . & 24.77 \\ 85471 . & 24.77 \\ 78640 . & 24.77 \\ 83350 . & 24.77 \\ 76457 . & 24.77 \\ 81046 . & 24.77 \\ 74.334 . & 24.77 \\ 78998 . & 24.77 \\ 72553 . & 24.77 \\ 75310 . & 24.36 \\ 667350 & 24.36 \\ 60946 . & 24.36 \\ 60101 . & 24.36 \\ 57592 . & 24.36 \\ 57432 . & 24.36 \\ 55959 . & 24.36\end{array}$

DATE 27 MAY E 1

IIME $11: 21: 28$

\begin{tabular}{|c|c|c|c|c|c|}
\hline $\begin{array}{l}\text { RICTION } \\
\text { FACTOR }\end{array}$ & $\begin{array}{l}\text { STANTON } \\
\text { NUMBER }\end{array}$ & $\begin{array}{l}\text { FILM H } \\
\text { KWIMZ-C }\end{array}$ & H* & $R(H \bullet)$ & $A R$ \\
\hline $\begin{array}{r}.01975 \\
.01982\end{array}$ & $\begin{array}{l}.00267 \\
.00268\end{array}$ & $\begin{array}{l}3.920 \\
3.778\end{array}$ & .0 & $\begin{array}{l}.00 \\
.00\end{array}$ & .0 \\
\hline .02017 & .00273 & 4.956 & .0 & .00 & .0 \\
\hline .02017 & .00273 & 3.878 & .0 & .00 & .0 \\
\hline .02040 & .00276 & 5.173 & .0 & .00 & .0 \\
\hline .02039 & .00275 & 3.989 & .0 & .00 & .0 \\
\hline .02793 & .00462 & 7.704 & 108.8 & 4.41 & 2.0 \\
\hline .04255 & .00465 & 6.523 & 87.1 & 4.42 & 2.0 \\
\hline .04253 & .00456 & 8.429 & 98.7 & 4.42 & 2.0 \\
\hline .04213 & .00465 & 6.850 & 83.8 & 4.40 & 2.0 \\
\hline .04231 & .00458 & 8.681 & 94.8 & 4.42 & 2.0 \\
\hline .04194 & .00466 & 6.974 & 80.3 & 4.42 & 2.0 \\
\hline .04240 & .00460 & 8.826 & 92.4 & 4.41 & 2.0 \\
\hline .04218 & .00469 & 7.052 & 79.5 & 4.42 & 2.0 \\
\hline .04272 & .00462 & 8.889 & 92.5 & 4.41 & 2.0 \\
\hline .04268 & .00471 & 7.101 & B1.4 & 4.42 & 2.0 \\
\hline .04321 & .00464 & 8.952 & 95.0 & 4.40 & 2.0 \\
\hline .04335 & .00472 & 7.121 & 85.9 & 4.42 & 2.0 \\
\hline .03711 & .00 .259 & 4.354 & 92.2 & 4.42 & 2.0 \\
\hline .02069 & .00266 & 4.626 & .0 & .00 & .0 \\
\hline .02113 & .00271 & 5.832 & .0 & .00 & .0 \\
\hline .02120 & .00272 & 3.769 & .0 & .00 & .0 \\
\hline .02141 & .00274 & 4.564 & .0 & .00 & .0 \\
\hline .02142 & .00274 & 3.651 & .0 & .00 & .0 \\
\hline .02155 & .00276 & 5.166 & .0 & .00 & .0 \\
\hline
\end{tabular}


CHANNEL RESULTS

CASE 1 CFTL BUNOLE - ONE SIXTH SECTION - FULLY ROUGH FLOH - 1/13/81

TIME $=.00000$ SECONOS PRESSURE $=9000.0 \mathrm{KPA}$

\begin{tabular}{|c|c|c|c|c|}
\hline $\begin{array}{l}\text { OISTANCE } \\
\text { (MM) }\end{array}$ & $\begin{array}{l}\text { OELTA-P } \\
\text { (KPA) }\end{array}$ & $\begin{array}{l}\text { TEMPERATURE } \\
\text { (OEG-C) }\end{array}$ & $\begin{array}{l}\text { DENSITY } \\
\text { (KG/MB) }\end{array}$ & $\begin{array}{l}F(O W \\
(K G / S)\end{array}$ \\
\hline $\begin{array}{r}.0 \\
186.6 \\
430.0\end{array}$ & $\begin{array}{r}.000 \\
-1.874 \\
-7.445\end{array}$ & $\begin{array}{l}325.1 \\
325.9 \\
327.1\end{array}$ & $\begin{array}{l}7.107 \\
7.096 \\
7.079\end{array}$ & $\begin{array}{l}.006713 \\
.006615 \\
.005331\end{array}$ \\
\hline 554.8 & -8.628 & 328.0 & 7.067 & .005655 \\
\hline $\begin{array}{l}714.6 \\
805.8\end{array}$ & $\begin{array}{l}-13.244 \\
-14.052\end{array}$ & $\begin{array}{l}328.9 \\
329.6\end{array}$ & $\begin{array}{l}7.054 \\
7.044\end{array}$ & $\begin{array}{l}.004791 \\
.005181\end{array}$ \\
\hline 991.2 & $\begin{array}{l}-17.434 \\
-25.275\end{array}$ & $\begin{array}{l}335.9 \\
343.9\end{array}$ & 6.970 & $\begin{array}{r}.006218 \\
.00527\end{array}$ \\
\hline $\begin{array}{l}1072.0 \\
1191.2\end{array}$ & $\begin{array}{l}-25.275 \\
-30.378\end{array}$ & $\begin{array}{l}343.9 \\
362.2\end{array}$ & $\begin{array}{l}6.878 \\
6.680\end{array}$ & $\begin{array}{l}.005271 \\
.006820\end{array}$ \\
\hline $1272 . n$ & -38.759 & 372.1 & 6.574 & .005496 \\
\hline 1391.2 & -44.2000 & 394.7 & 6.351 & .006927 \\
\hline 1472.0 & $-53.1 \geq 4$ & 405.9 & 6.244 & .005543 \\
\hline 1591.2 & -58.919 & 430.8 & 6.022 & .006959 \\
\hline $1672 . \pi$ & -68.419 & 442.0 & 5.925 & .005562 \\
\hline 1791.2 & -74.517 & 466.3 & 5.729 & .006969 \\
\hline $\begin{array}{l}1872.0 \\
1991.2\end{array}$ & $\begin{array}{l}-84.530 \\
-90.876\end{array}$ & $\begin{array}{l}476.0 \\
496.9\end{array}$ & $\begin{array}{l}5.651 \\
5.495\end{array}$ & .005572 \\
\hline 2072.0 & -101.299 & $\begin{array}{l}496.9 \\
504.1\end{array}$ & 5.440 & $\begin{array}{l}.006970 \\
.005575\end{array}$ \\
\hline 2179.1 & -106.617 & 514.0 & 5.368 & .006812 \\
\hline 2267.9 & -112.329 & 513.8 & 5.366 & .005257 \\
\hline 2402.8 & -118.306 & 514.2 & 5.360 & .004652 \\
\hline 2544.2 & -120.017 & 515.3 & 5.350 & .005281 \\
\hline 2636.3 & -125.455 & 515.7 & 5.346 & .004533 \\
\hline 2742.3 & -126.690 & $516 \cdot B$ & 5.336 & .005088 \\
\hline 2860.0 & -132.390 & 517.9 & 5.327 & .004526 \\
\hline
\end{tabular}

DATA FOR CHANNEL 15

$\begin{array}{lll}\text { REYNOLDS } & \text { AREA } & \text { FRICTION } \\ \text { NUABER } & \text { IMM2I } & \text { FACTOR } \\ 81781 . & 24.36 & .01975 \\ 80513 . & 24.36 & .01982 \\ 64789 . & 24.36 & .02083 \\ 68668 . & 24.36 & .02056 \\ 58114 . & 24.36 & .02136 \\ 62796 . & 24.36 & .02098 \\ 76104 . & 24.77 & .02810 \\ 63942 . & 24.77 & .04284 \\ 81085 . & 24.77 & .04309 \\ 64648 . & 24.77 & .04224 \\ 79578 . & 24.77 & .04296 \\ 62957 . & -04.77 & .04214 \\ 77109 . & 24.77 & .04298 \\ 60967 . & 24.77 & .04234 \\ 74655 . & 24.77 & .04329 \\ 59156 . & 24.77 & .04294 \\ 72612 . & 24.77 & .04373 \\ 57710 . & 24.77 & .04377 \\ 68729 . & 24.36 & .03754 \\ 53054 . & 24.36 & .02182 \\ 46933 . & 24.36 & .02246 \\ 53221 . & 24.36 & .02181 \\ 45668 . & 24.36 & .02261 \\ 51204 . & 24.36 & .02201 \\ 45509 . & 24.36 & .02263\end{array}$

DATE 27 MAY 81

TIME $11: 21: 28$

$\begin{array}{lcccc}\text { STANTON } & \text { FILMH } & \text { H. } & R I H+1 & \text { AR } \\ \text { NUMBER } & \text { KHIM2-C } & & & \\ .00267 & 3.820 & .0 & .00 & .0 \\ .00268 & 3.777 & .0 & .00 & .0 \\ .00281 & 3.193 & .0 & .00 & .0 \\ .00278 & 3.343 & .0 & .00 & .0 \\ .00288 & 2.940 & .0 & .00 & .0 \\ .00283 & 3.123 & .0 & .00 & .0 \\ .00465 & 9.347 & 106.0 & 4.42 & 2.0 \\ .00486 & 5.156 & 66.0 & 4.41 & 2.0 \\ .00461 & 10.043 & 97.3 & 4.41 & 2.0 \\ .00486 & 5.377 & 61.8 & 4.41 & 2.0 \\ .00464 & 10.281 & 92.9 & 4.40 & 2.0 \\ .00490 & 5.463 & 58.6 & 4.40 & 2.0 \\ .00466 & 10.423 & 89.8 & 4.42 & 2.0 \\ .00492 & 5.509 & 58.0 & 4.42 & 2.0 \\ .00469 & 10.466 & 89.1 & 4.42 & 2.0 \\ .00494 & 5.543 & 60.0 & 4.42 & 2.0 \\ .00471 & 10.542 & 90.5 & 4.42 & 2.0 \\ .00495 & 5.556 & 64.5 & 4.42 & 2.0 \\ .00263 & 4.456 & 84.7 & 4.41 & 2.0 \\ .00279 & 3.127 & .0 & .00 & .0 \\ .00287 & 2.845 & .0 & .00 & .0 \\ .00279 & 3.137 & .0 & .00 & .00 \\ .00289 & 2.788 & .00 & .00 & .00 \\ .00281 & 3.048 & .00 & .00 & .0 \\ .00289 & 2.785 & .0 & .00 & .00 \\ & & & & \end{array}$


CHANNEL RESULTS

CASE I CFTL BUNOLE - ONE SIXTH SEETION - FULLY POUGH FLOW - 1/13/B1

TIME = .0O0OO SECONOS PRESSJRE $=9000.0 \mathrm{KPA}$

$$
186.0
$$

186.6
430.0

554.8

714.6

805.8

991.2

1072.0

1191.2

272.0

1391.2

1591.2

672.0

1791.0

1872.0

1991.2

2072.0

2179.1

2267.9

2402.8

2544.2

2636.3

2742.3
2860.0

\begin{abstract}
DELTA-P
\end{abstract}
(KPA)

TE MPERATURE
(DEG-C)

$325 \cdot 1$

$-1.875$

$-7.443$

$-8.630$

$-13.240$

$-14.053$

$-17.441$

$-25.264$

$-30.395$

$-38.744$

$-44.219$

$-53.117$

$-58.939$

$-68.401$

-74.538
-84.512

$-90.809$

$-101.279$

$-106.641$

$-112.319$

$-118.303$

$-120.027$

$-125.449$

$-126.697$

$-132.391$
326.4

327.9

.328 .8

329.7

330.5

337.9

348.8

369.3

381.0

406.6

418.0
445.7

455.7

455.7

481.

489.4

510.4

515.7

524.3

519.1

517.6

518.3

518.9

519.9

521.2
DATA FOR CHANNEL 16

$\begin{array}{ll}\text { OENSITY } & \text { FLOH } \\ \text { (KG/M3) } & \text { (KG/S) } \\ 7.107 & .003338 \\ 7.090 & .003358 \\ 7.070 & .003374 \\ 7.057 & .003327 \\ 7.044 & .003370 \\ 7.033 & .003323 \\ 6.948 & .003309 \\ 6.825 & .003474 \\ 6.608 & .003316 \\ 6.487 & .003479 \\ 6.243 & .003324 \\ 6.137 & .003485 \\ 5.900 & .003333 \\ 5.815 & .003495 \\ 5.616 & .003347 \\ 5.553 & .003506 \\ 5.402 & .003361 \\ 5.362 & .003514 \\ 5.300 & .003376 \\ 5.331 & .003447 \\ 5.337 & .003317 \\ 5.330 & .003124 \\ 5.324 & .003267 \\ 5.316 & .003129 \\ 5.305 & .003205\end{array}$

REYNOLDS

NUMBER

100168.

100610 .

100939.

99408 .

100585 .

99106.

99553.

103240 .

96374.012 .45

99872.12 .45

92941.12 .45

96345.12 .45

89683.12 .45

93154.12 .45

87106. 12.45

90590.12 .45

85233. 12.45

88704.12 .45

83162 . 12.11

$85289 . \quad 12.11$

82191.12 .11

77363.12 .11

80853.12 .11
$77382 . \quad 12.11$
DATE 27 MAY DI

TIME $11: 21: 28$
RICTION
FACTOR

.01888

.01886

.01885

.01891

.01886

.01892

.04939

.10785

.10750

.10673

10681

10636

10680

.10667

.10737

.10754

.10839

.10882

.08479

.01957

.01973

.02000

.01981

.02000

.01990

$\begin{array}{lc}\text { STANTON } & \text { FILMH } \\ \text { NUMEER } & \text { KHIMZ C C } \\ \text {.00295 } & 4.221 \\ .00295 & 4.240 \\ .00295 & 4.256 \\ .00295 & 4.209 \\ .00295 & 4.290 \\ .00295 & 4.205 \\ .00548 & 7.728 \\ .00547 & 7.446 \\ .00555 & 7.872 \\ .00552 & 7.539 \\ .00560 & 7.976 \\ .00556 & 7.627 \\ .00564 & 8.069 \\ .00560 & 7.705 \\ .00567 & 8.139 \\ .00563 & 7.765 \\ .00569 & 8.197 \\ .00564 & 7.797 \\ .00292 & 4.317 \\ .00290 & 4.284 \\ .00293 & 4.319 \\ .00297 & 3.972 \\ .00294 & 4.110 \\ .00297 & 3.976 \\ .00295 & 4.128\end{array}$

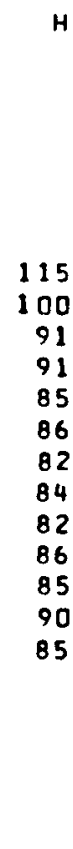

$R(H+)$

.00

.00
.00
.00
.00

.00

.00

4.37

4.37

4.37

4.37

4.37

4.37

4.37

4.37
4.37

4.37

4.37

4.37

4.37

4.37

4.37
.00

.00
.00

.00

.00

.00
AR

- 0

.0

.0

.0
.0

2.0

.

2.0

2.0

2.0

2.0

2.0

2.0

2.0

2.0

.0

.0

.0

.0
.0
.0 
CHANNEL RESULTS

CASE 1 CFIL BUNOLE - ONE SIXTH SECTION - FULLY ROUGH FLOH - 1/13/81

DATE 27 MAY RI

TIME

$11: 21: 28$

TIME $=.00000$ SECONDS PRESSURE $=9000.0 \mathrm{KPA}$

\begin{tabular}{|c|c|c|c|c|}
\hline $\begin{array}{l}\text { DISTANCE } \\
\text { (MM) }\end{array}$ & $\begin{array}{l}\text { DELTA -P } \\
\text { (KPA) }\end{array}$ & $\begin{array}{l}\text { TE MPER ATURE } \\
\text { (DEG -CI }\end{array}$ & $\begin{array}{l}\text { DENSITY } \\
\text { (KG/MZ) }\end{array}$ & $\begin{array}{l}F(O W \\
(K G / S)\end{array}$ \\
\hline 186 & $\begin{array}{r}.000 \\
-1.875\end{array}$ & 325.1 & 7.107 & .006713 \\
\hline 186.6 & -1.875 & 325.9 & 7.096 & .006588 \\
\hline 430.0 & -7.445 & 327.2 & 7.078 & .005844 \\
\hline 554.8 & -8.628 & 327.9 & 7.067 & .005893 \\
\hline 714.6 & -13.244 & 328.9 & 7.054 & .005441 \\
\hline 805.8 & -14.053 & 329.5 & 7.044 & .005532 \\
\hline 991.2 & -17.434 & 335.7 & 6.973 & .006264 \\
\hline 1072.0 & -25.277 & 343.9 & 6.878 & .006026 \\
\hline 1191.2 & -30.376 & 360.9 & 6.693 & .007005 \\
\hline 1272.0 & -38.762 & 371.4 & 6.581 & .006322 \\
\hline 1391.2 & -44.198 & 392.1 & 6.376 & .007152 \\
\hline 1472.0 & -53.138 & 404.3 & 6.258 & .006386 \\
\hline $\begin{array}{l}1591.2 \\
1672.0\end{array}$ & $\begin{array}{l}-58.917 \\
-68.423\end{array}$ & $\begin{array}{l}426.8 \\
439.4\end{array}$ & $\begin{array}{l}6.056 \\
5.046\end{array}$ & .007193 \\
\hline 1791.2 & -74.514 & 461.4 & $\begin{array}{l}5.946 \\
5.766\end{array}$ & $\begin{array}{l}.006404 \\
.007207\end{array}$ \\
\hline 1872.0 & -84.535 & 473.0 & 5.673 & .006406 \\
\hline 1991.2 & $=-90.873$ & 492.1 & 5.529 & .007205 \\
\hline 2072.0 & -101.304 & 501.3 & 5.459 & .006396 \\
\hline 2179.1 & -106.613 & 510.5 & 5.392 & .007047 \\
\hline $\begin{array}{l}2267.9 \\
2402.8\end{array}$ & $\begin{array}{l}-112.331 \\
-118.307\end{array}$ & $\begin{array}{l}511.8 \\
513.8\end{array}$ & $\begin{array}{l}5.380 \\
5.362\end{array}$ & $\begin{array}{r}.005950 \\
.005188\end{array}$ \\
\hline 2544.2 & -120.017 & 516.0 & 5.345 & .005130 \\
\hline 2636.3 & 25.456 & 517.4 & 5.334 & .004881 \\
\hline 2742.3 & -126.689 & 518.9 & 5.323 & .004885 \\
\hline 2860.0 & -132.390 & 521.1 & 5.306 & .004697 \\
\hline
\end{tabular}

DATA FOR CHANNEL 17

$\begin{array}{ll}\text { REYNOLOS } & \text { AREA } \\ \text { NUMBER } & \text { IMM2 I } \\ 81791 . & 24.36 \\ 80133 . & 24.36 \\ 71028 . & 24.36 \\ 71559 . & 24.36 \\ 65994 . & 24.36 \\ 67048 . & 24.36 \\ 76633 . & 24.77 \\ 73077 . & 24.77 \\ 83379 . & 24.77 \\ 74421 . & 24.77 \\ 82335 . & 24.77 \\ 72616 . & 24.77 \\ 80019 . & 24.77 \\ 70359 . & 24.77 \\ 77558 . & 24.77 \\ 68179 . & 24.77 \\ 753.32 . & 24.77 \\ 66377 . & 24.77 \\ 71315 . & 24.36 \\ 60119 . & 24.36 \\ 52351 . & 24.36 \\ 51670 . & 24.36 \\ 49102 . & 24.36 \\ 49073 . & 24.36 \\ 47004 . & 24.36 \\ & \end{array}$

FRICTION
FACTOR
.01975
.01984
.02040
.02036
.02074
.02067
.02808
.04276
.04273
.04223
.04254
.04215
.04265
.04242
.04287
.04294
.04336
.04365
.03734
.02119
.02189
.02196
.02222
.02223
.02244

(1)

$\begin{array}{ll}\text { STANTON } & \text { FILMH } \\ \text { NUMBER } & \text { KH/M2-C } \\ .00267 & 3.820 \\ .00268 & 3.765 \\ .00276 & 4.782 \\ .00275 & 3.512 \\ .00280 & 5.005 \\ .00279 & 3.610 \\ .00465 & 8.002 \\ .00472 & 6.273 \\ .00459 & 8.752 \\ .00471 & 6.574 \\ .00461 & 8.987 \\ .00474 & 6.680 \\ .00464 & 9.118 \\ .00477 & 6.738 \\ .00466 & 9.154 \\ .00479 & 6.770 \\ .00468 & 9.209 \\ .00481 & 6.773 \\ .00261 & 4.277 \\ .00272 & 4.117 \\ .00280 & 5.450 \\ .00281 & 3.073 \\ .00284 & 3.955 \\ .00284 & 2.953 \\ .00286 & 4.634\end{array}$

$R(H+)$

$A R$

.00
.00
.00
.00
.00
.00
4.40
4.41
4.41
4.42
4.41
4.41
4.40
4.41
4.42
4.41
4.42
4.41
4.41
.00
.00
.00
.00
.00
.00


CHANNEL RESULTS

CASE

TIME $=.00000$ SECONOS PRESSURE $=0,00 J .0 \mathrm{KPA}$

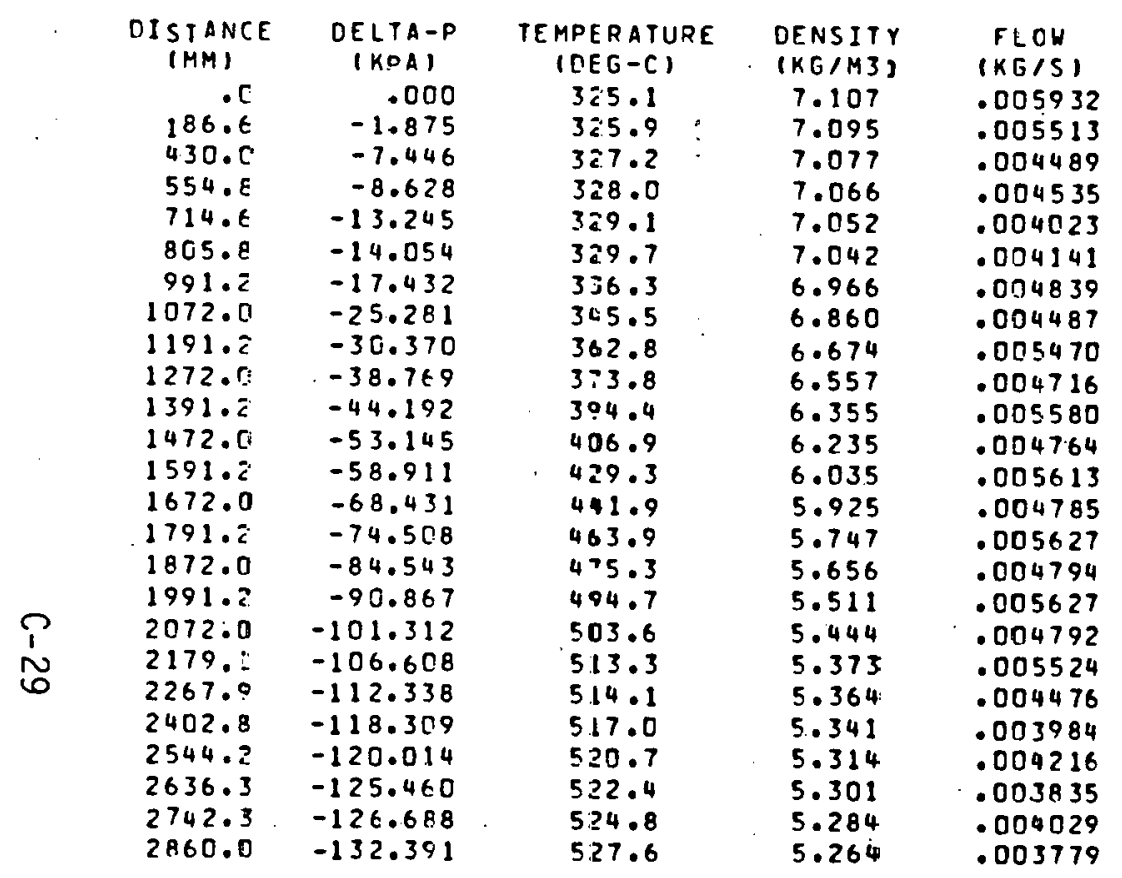

DATE 27 MAY 81 TIME

$11: 21: 28$

OATA FOR CHANNEL 18

$\begin{array}{ccccc}\text { PEYNOLDS } & \text { AREA } & \text { FRICTION } & \text { STANTON } & \text { FILMH } \\ \text { NUMEER } & \text { (MM2) } & \text { FACTOR } & \text { NUMBER } & \text { KHIMZ-C } \\ 58867 . & 21.53 & .02130 & .00277 & 3.760 \\ 54650 . & 21.53 & .02167 & .00282 & 3.741 \\ 44438 . & 21.53 & .02276 & .00295 & 3.192 \\ 44847 . & 21.53 & .02271 & .00294 & 3.216 \\ 39741 . & 21.53 & .02337 & .00303 & 2.933 \\ 40876 . & 21.53 & .02322 & .00301 & 2.999 \\ 48213 . & 21.87 & .03223 & .00519 & 8.617 \\ 44254 . & 21.87 & .05044 & .00534 & 5.448 \\ 52944 . & 21.87 & .05049 & .00512 & 9.445 \\ 45109 . & 21.87 & .04960 & .00533 & 5.708 \\ 52233 . & 21.87 & .05029 & .00514 & 9.699 \\ 44032 . & 21.87 & .04962 & .00537 & 5.813 \\ 50736 . & 21.87 & .05042 & .00517 & 9.853 \\ 42721 . & 21.87 & .04981 & .00539 & 5.865 \\ 49204 . & 21.87 & .05078 & .00520 & 9.897 \\ 41481 . & 21.87 & .05034 & .00541 & 5.894 \\ 47839 . & 21.87 & .05131 & .00523 & 9.970 \\ 40420 . & 21.87 & .05126 & .00542 & 5.904 \\ 45426 . & 21.53 & .04354 & .00279 & 3.854 \\ 36781 . & 21.53 & .02381 & .00292 & 3.153 \\ 32650 . & 21.53 & .02451 & .00300 & 2.882 \\ 34444 . & 21.53 & .02419 & .00296 & 3.010 \\ 31283 . & 21.53 & .02477 & .00303 & 2.798 \\ 32802 . & 21.53 & .02448 & .00299 & 2.906 \\ 30695 . & 21.53 & .02488 & .00304 & 2.766\end{array}$

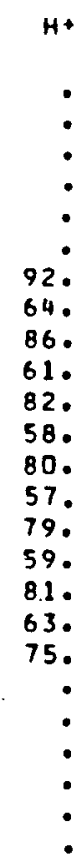

$R(H+)$

$A R$

$\begin{array}{ll}.00 & 100 \\ .00 & 10 \\ .00 & .0 \\ .00 & .0 \\ .00 & .0 \\ .00 & .0 \\ 4.41 & 2.0 \\ 4.39 & 2.0 \\ 4.42 & 2.0 \\ 4.41 & 2.0 \\ 4.42 & 2.0 \\ 4.39 & 2.0 \\ 4.41 & 2.0 \\ 4.40 & 2.0 \\ 4.41 & 2.0 \\ 4.42 & 2.0 \\ 4.41 & 2.0 \\ 4.42 & 2.0 \\ 4.41 & 2.0 \\ .00 & .00 \\ .00 & .0 \\ .00 & .0 \\ .00 & .0 \\ .00 & .0 \\ .00 & .0\end{array}$


CHANNEL RESUL TS

CASE 1 CFTL BUNDLE - ONE SIXTH SECTION - FULLY ROUGH FLON - $1 / 13 / 81$

TIME $=.00000$ SECONOS PRESSURE $=9000.0 \mathrm{KPA}$

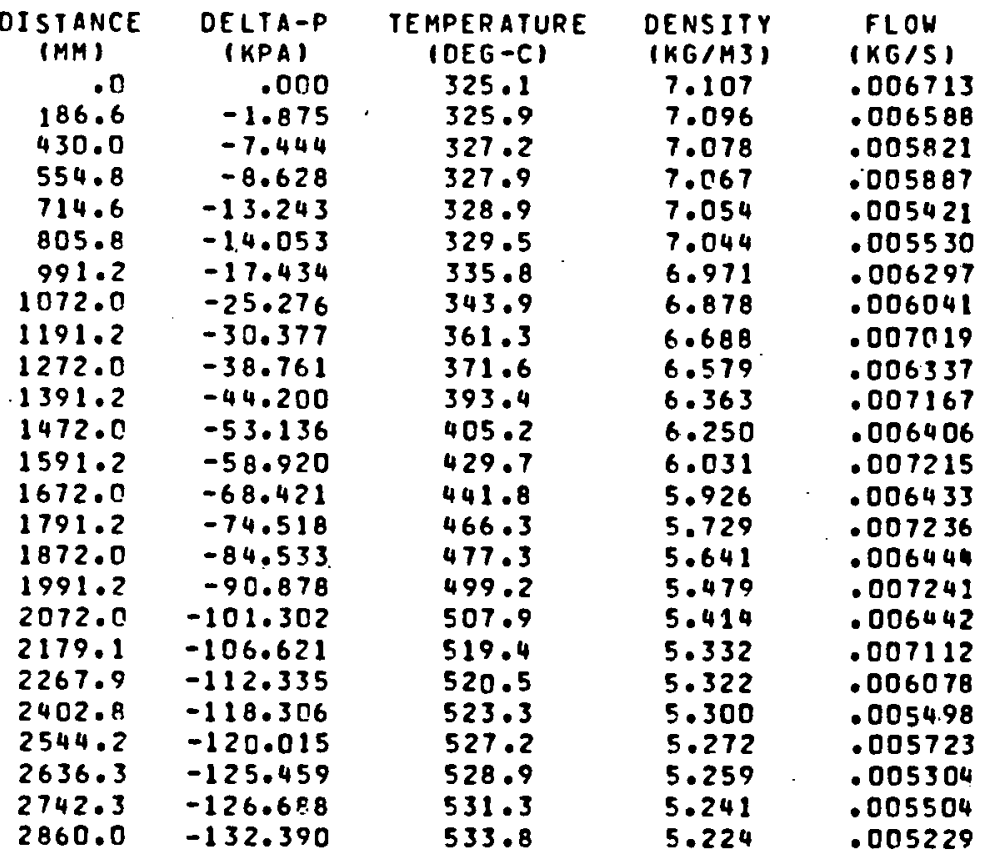

DATA =OR CHANNEL 19

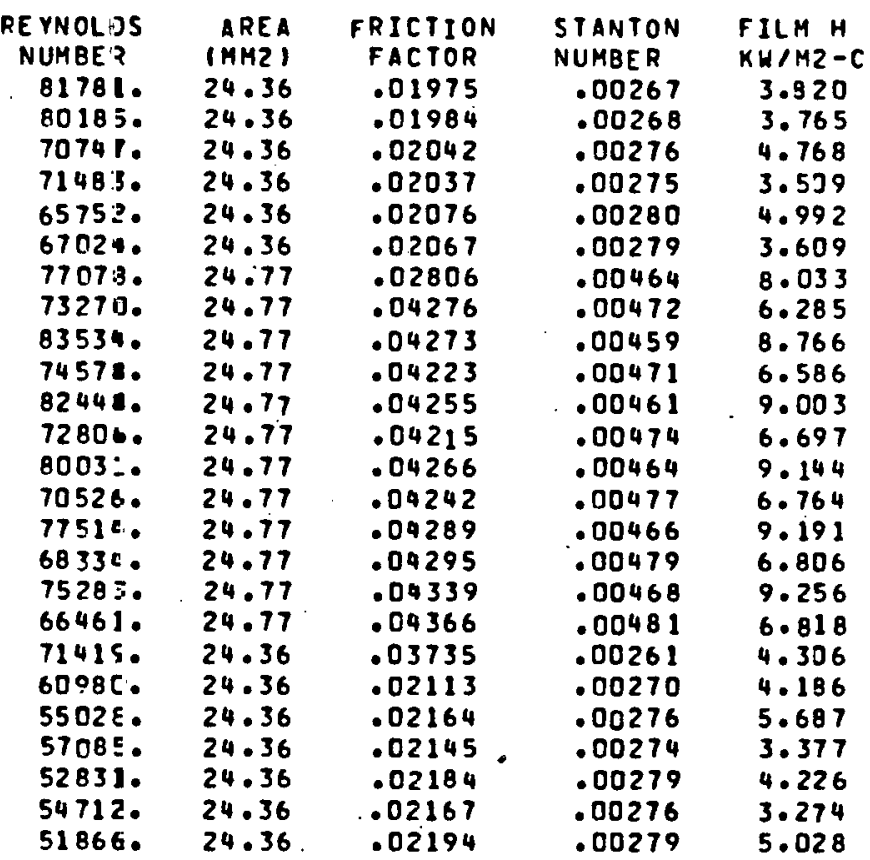

$R(H+)$

AR

.0
.0
.0
.0
.0
.0
106.4
80.5
96.9
77.0
92.9
73.4
90.0
72.6
89.5
74.3
91.4
78.2
87.6
.0
.0
.0
.0
.0
.0


CHANNEL RESUL IS

TIME $=.00000$ SECONDS PRESSURE $=9000.0 \mathrm{KPA}$

DELTA-P TEMPERATURE DENSITY

\begin{tabular}{|c|c|c|c|c|}
\hline $\begin{array}{l}\text { I STANCE } \\
\text { (MM) }\end{array}$ & OELTA-P & $\begin{array}{l}\text { TEMPER ATURE } \\
\text { (DEG-C) }\end{array}$ & $\begin{array}{l}\text { DENS ITY } \\
\text { |KG/MS| }\end{array}$ & $\begin{array}{l}F L O W \\
\text { (KG/S) }\end{array}$ \\
\hline .0 & .000 & 325.1 & 7.107 & .003338 \\
\hline 186.6 & -1.875 & 326.4 & 7.090 & $.00 \pm 359$ \\
\hline 430.0 & -7.442 & 327.9 & 7.070 & .003401 \\
\hline 554.8 & -8.630 & 328.9 & 7.056 & .003353 \\
\hline 714.6 & -13.239 & 329.8 & 7.043 & .003411 \\
\hline 805.8 & -14.053 & 330.5 & 7.033 & .003368 \\
\hline 991.2 & -17.441 & 339.0 & 6.948 & .003342 \\
\hline 1072.0 & -25.263 & 348.8 & 6.825 & .003486 \\
\hline 1191.2 & $-30.3 \div 5$ & 369.2 & 6.608 & .003329 \\
\hline 1272.0 & -38.742 & 381.0 & 6.487 & .003482 \\
\hline 1391.2 & -44.220 & 406.9 & 6.239 & .003330 \\
\hline 1472.0 & -53.115 & 418.7 & 6.131 & .003486 \\
\hline 1591.2 & -58.941 & 447.6 & 5.885 & .003334 \\
\hline 1672.0 & -68.399 & 458.0 & 5.797 & .003491 \\
\hline 1791.2 & -74.541 & 486.2 & 5.581 & .003338 \\
\hline 1872.0 & $-84.5 C 9$ & 494.3 & 5.519 & .003492 \\
\hline 1991.2 & -90.902 & 518.6 & 5.347 & .003342 \\
\hline 2072.0 & -101.277 & 523.6 & 5.309 & .003491 \\
\hline 2179.1 & -106.647 & 535.3 & 5.229 & .003352 \\
\hline 2267.9 & -112.323 & 530.2 & 5.259 & .003430 \\
\hline 2402.8 & $-118.3 \Gamma 2$ & 530.4 & 5.254 & .003387 \\
\hline 2544.2 & -120.022 & 532.6 & 5.238 & .003316 \\
\hline 2636.3 & -125.451 & 533.6 & 5.229 & .003375 \\
\hline $2742 \cdot 3$ & -126.693 & 534.8 & 5.219 & .003306 \\
\hline 2860.0 & $-132 \cdot 390$ & 536.7 & 5.205 & .003346 \\
\hline
\end{tabular}

DATA FOR CHANNEL 20

$\begin{array}{ll}\text { REYNOLOS } & \text { AREA } \\ \text { NUMBER } & \text { IMM2 } \\ 100168 . & 12.11 \\ 100640 . & 12.11 \\ 101730 . & 12.11 \\ 100181 . & 12.11 \\ 101829 . & 12.11 \\ 100453 . & 12.11 \\ 100515 . & 12.45 \\ 103618 . & 12.45 \\ 96773 . & 12.45 \\ 99965 . & 12.45 \\ 93071 . & 12.45 \\ 96302 . & 12.45 \\ 89529 . & 12.45 \\ 9282.0^{\circ} & 12.45 \\ 86483 . & 12.45 \\ 89834 . & 12.45 \\ 84133 . & 12.45 \\ 87528 . & 12.45 \\ 81794 . & 12.11 \\ 84060 . & 12.11 \\ 83003 . & 12.11 \\ 81110 . & 12.11 \\ 82460^{\circ} & 12.11 \\ 80718 . & 12.11 \\ 81544 . & 12.11\end{array}$

DATE 27 MAY 81

TIME $11: 21: 28$

FRICIION
FACTOR
.01888
.01886
.01881
.01888
.01881
.01887
.04937
.10786
.107 .51
.10673
.10682
.10636
.10681
.10668
.10740
.10756
.10842
.10884
.08483
.01963
.01969
.01979
.01972
.01981
.01977

STANTON
NUMBER
.00295
.00295
.00294
.00295
.002 .94
.00295
.00547
.00547
.00555
.00552
.00560
.00557
.00564
.00560
.00568
.00563
.00570
.00565
.00292
.00290
.00291
.00293
.00291
.00293
.00292

.

FILMH
$K W / M 2-C$
4.221
4.241
4.281
4.234
4.331
4.249
7.783
7.466
7.896
7.544
7.987
7.629
8.073
7.701
8.131
7.750
8.175
7.772
4.291
4.265
4.386
4.155
4.211
4.146
4.263

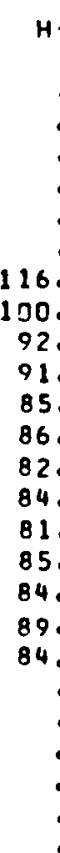

$R(H \bullet)$

.0
.0
.0
.0
.0
.0
2.0
2.0
2.0
2.0
2.0
2.0
2.0
2.0
2.0
2.0
2.0
2.0
2.0
.00
.00
.0
.0
.0
.0


CHANNEL RESULTS

CASE 1 CFTL BUNOLE - ONE SIXTH SECTION - FULLY ROUGH FLOH - 1/13/81

TIME $=.00000$ SECONDS PRESSURE $=9000.0 \mathrm{KPA}$

\begin{tabular}{|c|c|c|c|c|}
\hline $\begin{array}{l}\text { ISTANCE } \\
\text { IMMI }\end{array}$ & $\begin{array}{l}\text { DELTA-P } \\
\text { (KPA) }\end{array}$ & $\begin{array}{l}\text { TEMPER A TURE } \\
\text { (DEG-C) }\end{array}$ & $\begin{array}{l}\text { DENSITY } \\
\text { IKG/MZ }\end{array}$ & $\begin{array}{l}\text { FLOW } \\
\text { (KG/S) }\end{array}$ \\
\hline $\begin{array}{r}.0 \\
186.6 \\
430.0 \\
554.8 \\
714.6\end{array}$ & $\begin{array}{r}.000 \\
-1.875 \\
-7.444 \\
-8.628 \\
-13.243\end{array}$ & $\begin{array}{l}325.1 \\
325.9 \\
327.1 \\
328.0 \\
328.9\end{array}$ & $\begin{array}{l}7.107 \\
7.096 \\
7.078 \\
7.066 \\
7.053\end{array}$ & $\begin{array}{l}.006713 \\
.006617 \\
.005356 \\
.005683 \\
.004830\end{array}$ \\
\hline $\begin{array}{r}805.8 \\
991.2\end{array}$ & $\begin{array}{l}-14.052 \\
-17.435\end{array}$ & $\begin{array}{l}329.6 \\
335.8\end{array}$ & $\begin{array}{l}7.043 \\
6.972\end{array}$ & $\begin{array}{l}.005231 \\
.006253\end{array}$ \\
\hline 1072.0 & -25.275 & 343.8 & 6.879 & .005284 \\
\hline $\begin{array}{l}1191.2 \\
1272.0\end{array}$ & $\begin{array}{l}-30.380 \\
-38.758\end{array}$ & $\begin{array}{l}361.7 \\
371.8\end{array}$ & $\begin{array}{l}6.684 \\
6.578\end{array}$ & .006852 \\
\hline 1391.2 & -44.204 & 394.1 & 6.357 & .006967 \\
\hline 1472.0 & -53.133 & 405.7 & 6.245 & .005559 \\
\hline $\begin{array}{l}1591.2 \\
1672.0\end{array}$ & $\begin{array}{l}-58.925 \\
-68.418\end{array}$ & $\begin{array}{r}430.9 \\
442.9\end{array}$ & $\begin{array}{l}6.022 \\
5.918\end{array}$ & $\begin{array}{l}.007006 \\
.005577\end{array}$ \\
\hline 1791.2 & $\begin{array}{l}-74.524 \\
-84.520\end{array}$ & 468.2 & 5.714 & .007019 \\
\hline $\begin{array}{l}1872.0 \\
1991.2\end{array}$ & $\begin{array}{l}-84.529 \\
-90.885\end{array}$ & $\begin{array}{l}479.2 \\
502.2\end{array}$ & $\begin{array}{l}5.627 \\
5.458\end{array}$ & - 005581 \\
\hline 2072.0 & -101.298 & 511.1 & $\begin{array}{l}3.438 \\
5.393\end{array}$ & .005573 \\
\hline 2179.1 & -106.629 & 523.9 & 5.302 & .006838 \\
\hline 2267.9 & -112.336 & 525.2 & 5.291 & .005240 \\
\hline 2402.8 & -118.307 & 529.8 & 5.258 & .004622 \\
\hline 2544.2 & -120.016 & 534.9 & 5.223 & .005402 \\
\hline 2636.3 & 3.462 & 536.5 & 5.210 & .004540 \\
\hline $\begin{array}{l}2742.3 \\
2860.0\end{array}$ & $\begin{array}{l}-126.689 \\
-132.391\end{array}$ & $\begin{array}{l}539.1 \\
541.1\end{array}$ & $\begin{array}{l}5.192 \\
5.178\end{array}$ & .005190 \\
\hline 20000 & -1220.24 & - 1 & 178 & .004530 \\
\hline
\end{tabular}

DATA FOQ CHANNEL 21

$\begin{array}{lll}\text { REYNOLOS } & \text { AREA } & \text { FRICIJON } \\ \text { NUMBER } & \text { I4H2) } & \text { FACIOR } \\ 81781 . & 24.36 & .01975 \\ 80535 . & 24.36 & .01982 \\ 65099 . & 24.36 & .02081 \\ 69003 . & 24.36 & .02053 \\ 58581 . & 24.36 & .02132 \\ 63396 . & 24.36 & .02094 \\ 76540 . & 24.77 & .02811 \\ 64106 . & 24.77 & .04284 \\ 81509 . & 24.77 & .04308 \\ 64924 . & 24.77 & .04223 \\ 80092 . & 24.77 & .04294 \\ 63157 . & 24.77 & .04214 \\ 77622 . & 24.77 & .04296 \\ 61081 . & 24.77 & .04244 \\ 75055 . & 24.77 & .04328 \\ 59077 . & 24.77 & .04296 \\ 72749 . & 24.77 & .04374 \\ 57342 . & 24.77 & .04382 \\ 68396 . & 24.36 & .03758 \\ 52360 . & 24.36 & .02189 \\ 46000 . & 24.36 & .02257 \\ 53529 . & 24.36 & .02178 \\ 44929 . & 24.36 & .02270 \\ 51242 . & 24.36 & .02200 \\ 44659 . & 24.36 & .02273\end{array}$

$\begin{array}{lr}\text { STANTON } & \text { FILMH } \\ \text { NUMBER } & \text { KW/M2-C } \\ .00267 & 3.320 \\ .00268 & 3.777 \\ .00281 & 3.205 \\ .00277 & 3.356 \\ .00288 & 2.958 \\ .00283 & 3.146 \\ .00465 & 9.395 \\ .00486 & 5.166 \\ .00461 & 10.075 \\ .00486 & 5.387 \\ .00463 & 10.322 \\ .00489 & 5.475 \\ .00466 & 10.472 \\ .00492 & 5.529 \\ .00469 & 10.523 \\ .00494 & 5.553 \\ .00471 & 10.603 \\ .00496 & 5.562 \\ .00263 & 4.467 \\ .00279 & 3.117 \\ .00287 & 2.829 \\ .00277 & 3.190 \\ .00289 & 2.789 \\ .00280 & 3.092 \\ .00289 & 2.784\end{array}$

(1)

\begin{tabular}{l}
$H$ \\
9 \\
9 \\
9 \\
9 \\
5 \\
9 \\
5 \\
58 \\
89 \\
60.7 \\
9 \\
64.08 \\
84.6 \\
\hline
\end{tabular}

$R(H+)$ 
CHANNEL RESULTS

CASE

TIME = .00000 SECONOS

$\begin{array}{ccccc}\text { OISTANCE } & \text { DELTA-P } & \text { TEMPERATURE } & \text { OENSITY } & \text { FLON. } \\ \text { (MM) } & \text { (KPA) } & \text { (OEG-C) } & \text { (KG/M3) } & \text { (KG/S) } \\ 180 & .0 C 0 & 325.1 & 7.107 & .006713 \\ 186.6 & -1.875 & 325.9 & 7.096 & .006621 \\ 430.0 & -7.442 & 327.2 & 7.078 & .006148 \\ 554.8 & -8.630 & 327.9 & 7.067 & .006175 \\ 714.6 & -13.240 & 328.9 & 7.054 & .005881 \\ 805.8 & -14.053 & 329.5 & 7.045 & .005941 \\ 991.2 & -17.440 & 335.5 & 6.976 & .006566 \\ 1072.0 . & -25.265 & 343.3 & 6.885 & .006515 \\ 1191.2 & -30.391 & 360.1 & 6.701 & .007275 \\ 1272.0 & -38.746 & 370.3 & 6.593 & .006831 \\ 1391.2 & -44.217 & 392.6 & 6.371 & .007424 \\ 1472.0 & -53.119 & 404.5 & 6.257 & .006913 \\ 1591.2 & -58.939 & 431.2 & 6.019 & .007452 \\ 1672.0 & -68.402 & 443.4 & 5.913 & .006932 \\ 1791.2 & -74.540 & 471.4 & 5.690 & .007444 \\ 1872.0 & -84.513 & 482.6 & 5.602 & .006925 \\ 1991.2 & -90.902 & 508.7 & 5.414 & .007422 \\ 2072.0 & -101.280 & 517.5 & 5.349 & .006908 \\ 2179.1 & -106.648 & 532.9 & 5.244 & .007323 \\ 2267.9 & -112.330 & 533.6 & 5.237 & .006574 \\ 2402.8 & -118.303 & 538.8 & 5.200 & .006298 \\ 2544.2 & -120.020 & 543.6 & 5.168 & .006625 \\ 2636.3 & -125.457 & 544.5 & 5.160 & .006205 \\ 2742.3 & -126.692 & 546.2 & 5.148 & .006513 \\ 2860.0 & -132.391 & 547.0 & 5.141 & .006285\end{array}$

DATA FOR CHANNEL 22

$\begin{array}{ccc}\text { REYNOLOS } & \text { AREA } & \text { FRICIION } \\ \text { NUMBER } & \text { IMM? } & \text { FACIOR } \\ \text { B1781. } & 24.36 & .01975 \\ 80583 . & 24.36 & .01982 \\ 74720 . & 24.36 & .02016 \\ 74979 . & 24.36 & .02015 \\ 71342 . & 24.36 & .02038 \\ 72018 . & 24.36 & .02033 \\ 80401 . & 24.77 & .02786 \\ 79087 . & 24.77 & .04263 \\ 86684 . & 24.77 & .04252 \\ 80509 . & 24.77 & .04212 \\ 85473 . & 24.77 & .04233 \\ 78633 . & 24.77 & .04196 \\ 82546 . & 24.77 & .04246 \\ 75875 . & 24.77 & .04224 \\ 79366 . & 24.77 & .04284 \\ 73085 . & 24.77 & .04278 \\ 76523 . & 24.77 & .04327 \\ 70678 . & 24.77 & .04349 \\ 72691 . & 24.36 & .03729 \\ 65219 . & 24.36 & .02080 \\ 62203 . & 24.36 & .02103 \\ 65171 . & 24.36 & .02080 \\ 60991 . & 24.36 & .02112 \\ 63925 . & 24.36 & .02090 \\ 61645 . & 24.36 & .02107\end{array}$

QATE 27 MAY 8

TIME $11: 21: 28$ NUMBER KH/MZ-C 00267

.00268

.00273

0.00275

.00275

00460

00456

00464

.00458

.00469

00464

00472

00467

00475

00259

.00265

00268

.00265

.00269

00269

.00268
$K H / M 2-C$
3.320

3.320
3.779

4.960

3.394

5.194

4.024

7.799

6.571

8. 484

6.880

8.710

6.996

8.831

7.063

8.871

7.099

8.905

7.109

4.311
4.623

6.025

4.102

4.859

4.859

4.057
5.641
H

:

110.7

98.0

84.3

95.0

80.5

91.8

910

80.5

92.3

84.1

89.4

.0

.0
.0
.0
A R

$\begin{array}{ll}.00 & .0 \\ .00 & .0 \\ .00 & .0 \\ .00 & .0 \\ .00 & .0 \\ .00 & .0 \\ 4.41 & 2.0 \\ 4.40 & 2.0 \\ 4.42 & 2.0 \\ 4.40 & 2.0 \\ 4.42 & 2.0 \\ 4.42 & 2.0 \\ 4.41 & 2.0 \\ 4.42 & 2.0 \\ 4.40 & 2.0 \\ 4.42 & 2.0 \\ 4.42 & 2.0 \\ 4.41 & 2.0 \\ 4.42 & 2.0 \\ .00 & .0 \\ .00 & .0 \\ .00 & .0 \\ .00 & .0 \\ .00 & .0 \\ .00 & .0\end{array}$


CHANNEL RESULTS$$
\text { CASE } 1
$$

TIME $=.00000$ SECONOS PRESSURE $=9000.0 \mathrm{KPA}$

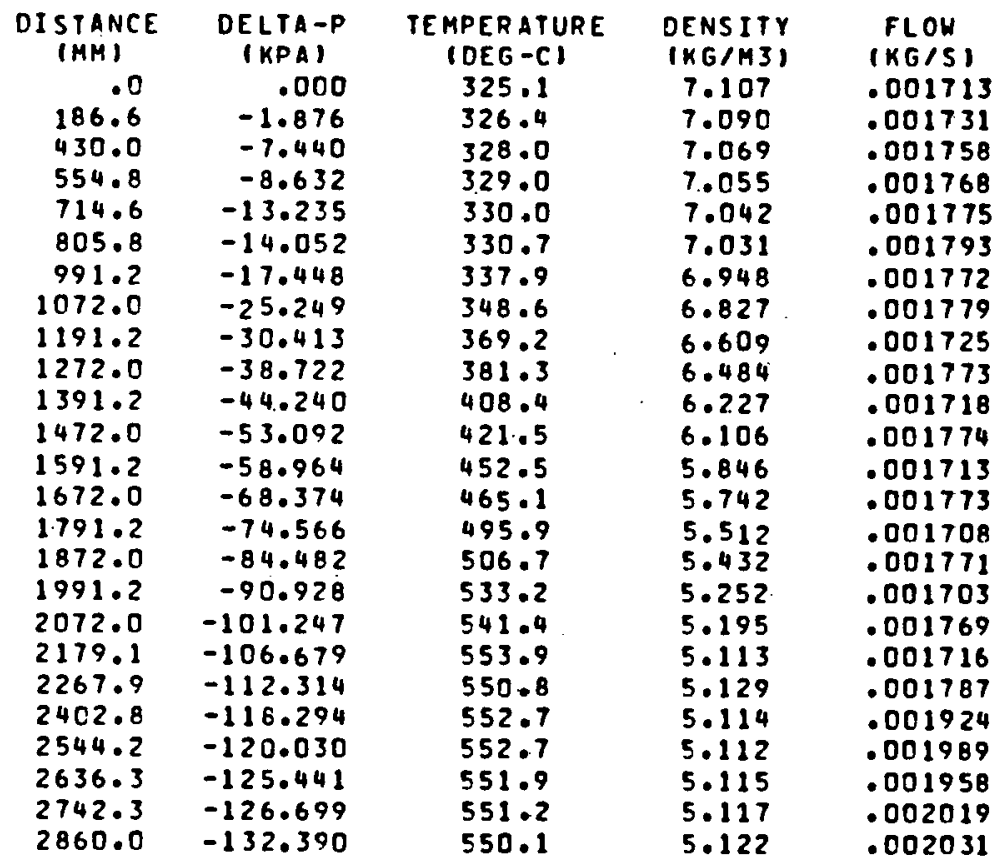

OATA FOR CHANNEL 23

$\begin{array}{lll}\text { REYNCLOS } & \text { AREA } & \text { FRICTION } \\ \text { NUMEER } & \text { IMMEI } & \text { FACIOR } \\ 102823 . & 6.22 & .01877 \\ 103763 . & 6.22 & .01873 \\ 105162 . & 6.22 & .01868 \\ 1051 & .0186 \\ 105646 . & 6.22 & .01866 \\ 105968 . & 6.22 & .01865 \\ 106504 . & 6.22 & .01861 \\ 106606 . & 6.39 & .04924 \\ 105763 . & 6.39 & .10797 \\ 100310 . & 6.39 & .10785 \\ 101748 . & 6.39 & .10687 \\ 95910 . & 6.39 & .10720 \\ 97757 . & 6.39 & .10653 \\ 91535 . & 6.39 & .10720 \\ 93698 . & 6.39 & .10687 \\ 87729 . & 6.39 & .10776 \\ 90120 . & 6.39 & .10774 \\ 84634 . & 6.39 & .10873 \\ 87356 . & 6.39 & .10900 \\ 82432 . & 6.22 & .08493 \\ 86052 . & 6.22 & .01953 \\ 92523 . & 6.22 & .01921 \\ 95658 . & 6.22 & .01907 \\ 942.57 . & 6.22 & .01914 \\ 97227 . & 6.22 & .01900 \\ 97834 . & 6.22 & .01898\end{array}$

$\begin{array}{lc}\text { STANTON } & \text { FILMH } \\ \text { NUMBER } & \text { KHAM2-C } \\ .00295 & 4.221 \\ .00295 & 4.255 \\ .00293 & 5.020 \\ .00293 & 4.415 \\ .00293 & 5.272 \\ .00292 & 4.616 \\ .00540 & 8.528 \\ .00544 & 7.709 \\ .00550 & 8.494 \\ .00550 & 7.776 \\ .00556 & 8.571 \\ .00555 & 7.865 \\ .00562 & 8.645 \\ .00559 & 7.934 \\ .00566 & 8.684 \\ .00563 & 7.980 \\ .00569 & 8.719 \\ .00565 & 8.003 \\ .00292 & 4.404 \\ .00289 & 4.746 \\ .00284 & 5.879 \\ .00282 & 4.811 \\ .00283 & 5.342 \\ .00281 & 4.873 \\ .00281 & 5.905\end{array}$

$R(H+1$

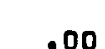

AR

.00

.00

.00

.00
.00

4.37

4.37

4.37

4.37$$
4.37
$$

4.37

4.37

4.37
4.37

4.37
4.37

4.37

4.37
4.37

.00

.00
.00

.00

.00

.00
.00 
CHANNEL RESULTS

CASE

IIME $=.00000$ SECONOS PRESSURE $=9000.0 \mathrm{KPA}$

CISTANCE DELTA-P
(MM)
186.5
430.5

554.9

714.5

805.3

991.2

1072.3

1191.2

1272.0

1472.0

1591.2

1672.0

1791.2

1872.0

2072.0

(KPA)$$
-1.878
$$

$-7.436$

$-7.436$

$-13.228$

$-14.047$

$-17.461$

$-25.222$

$-38.679$

$-44.292$

$-53.044$

$-59.019$

-68.323
-74.624

-74.624
-84.429

$-90.991$

$-101.192$

$-106.747$

2179.1

2267.9

2544.2

2636.3

$-112.28$

$-118.282$

-120.054
-125.426

$-126.714$

$-132.392$
OENSITY FLOH (KG/MB)

(DEG-C)

325.1

325.6

326.7

327.6

328.5

329.3

340.1

357.4

368.3

393.9

407.4

437.7

451.8

482.4

495.2

522.2

531.9

547.0

548.0

549.8

550.6

550.0

549.9

549.1
7.100

7.084

7.071

7.059

7.047

6.991

6.920

6.729

6.61 ?

6.355

6.230

$5.96=$

5.846

5.609

5.512

5.320

5.256

5.156

5.140

5.132

5.125

5.126

5.125

5.128
.001610

.001590

001384

001475

.001309

.001426

.001469

001288

.001375

. 001260

001369

001259

001369

.001258

.001367

001256

001365

001254

.001361

001207

.001258

.001487

.001283

.001485

DATE

27 MAY $\&$

TIME

$11: 21: 28$

OATA FOP CHANNEL 24

$\begin{array}{ccc}\text { PEYNOLDS } & \text { AREA } & \text { FRICIION } \\ \text { NUMBER } & \text { (MHE) } & \text { FACTOR } \\ 80183 . & 5.84 & .01984 \\ 79147 . & 5.84 & .01990 \\ 68803 . & 5.84 & .02055 \\ 73279 . & 5.84 & .02025 \\ 64945 . & 5.84 & .02082 \\ 70690 . & 5.84 & .02042 \\ 73679 . & 6.06 & .05461 \\ 64154 . & 6.06 & .12434 \\ 67219 . & 6.06 & .12434 \\ 60876 . & 6.06 & .12434 \\ 64353 . & 6.06 & .12434 \\ 58404 . & 6.06 & .12434 \\ 61600 . & 6.06 & .12434 \\ 55866 . & 6.06 & .12434 \\ 59006 . & 6.06 & .12434 \\ 53583 . & 6.06 & .12434 \\ 56883 . & 6.06 & .12434 \\ 51823 . & 6.06 & .12434 \\ 54602 . & 5.84 & .09647 \\ 48384 . & 5.84 & .02230 \\ 50328 . & 5.84 & .02210 \\ 59447 . & 5.84 & .02125 \\ 51342 . & 5.84 & .02199 \\ 59424 . & 5.84 & .02125 \\ 52981 . & 5.84 & .02183\end{array}$

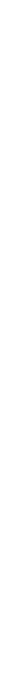

$\begin{array}{lc}\text { STANTON } & \text { FILMH } \\ \text { NUMBER } & \text { KH/M2-C } \\ .00301 & 4.303 \\ .00302 & 4.262 \\ .00312 & 3.830 \\ .00307 & 4.023 \\ .00316 & 3.669 \\ .00309 & 3.919 \\ .00586 & 10.412 \\ .00603 & 6.405 \\ .00597 & 9.936 \\ .00609 & 6.338 \\ .00603 & 10.018 \\ .00615 & 6.380 \\ .00608 & 10.149 \\ .00621 & 6.424 \\ .00614 & 10.211 \\ .00626 & 6.463 \\ .00619 & 10.313 \\ .00630 & 6.491 \\ .00309 & 4.242 \\ .00318 & 3.410 \\ .00315 & 3.519 \\ .00303 & 4.002 \\ .00314 & 3.574 \\ .00303 & 3.999 \\ .00311 & 3.659\end{array}$

$(\mathrm{H}+)$

.0

.0

.0

.0

2.0

.37

$4.37 \quad 2.0$

$\begin{array}{ll}4.37 & 2.0 \\ 4.37 & 2.0\end{array}$

$\begin{array}{ll}4.37 & 2.0 \\ 4.37 & 2.0\end{array}$

4.372 .0

$4.37 \quad 2.0$

$4.37 \quad 2.0$

$4.37 \quad 2.0$

$4.37 \quad 2.0$

$4.37 \quad 2.0$

$4.37 \quad 2.0$

4.372 .0

$.00 \quad .0$

.00

.00

.00

.00

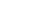


CHAMNEL RESULIS

CASE

RESULTS

IIME $=.00000$ SECONOS

PRESSURE $=9000.0 \mathrm{KPA}$

TEMPERATURE OENSITY

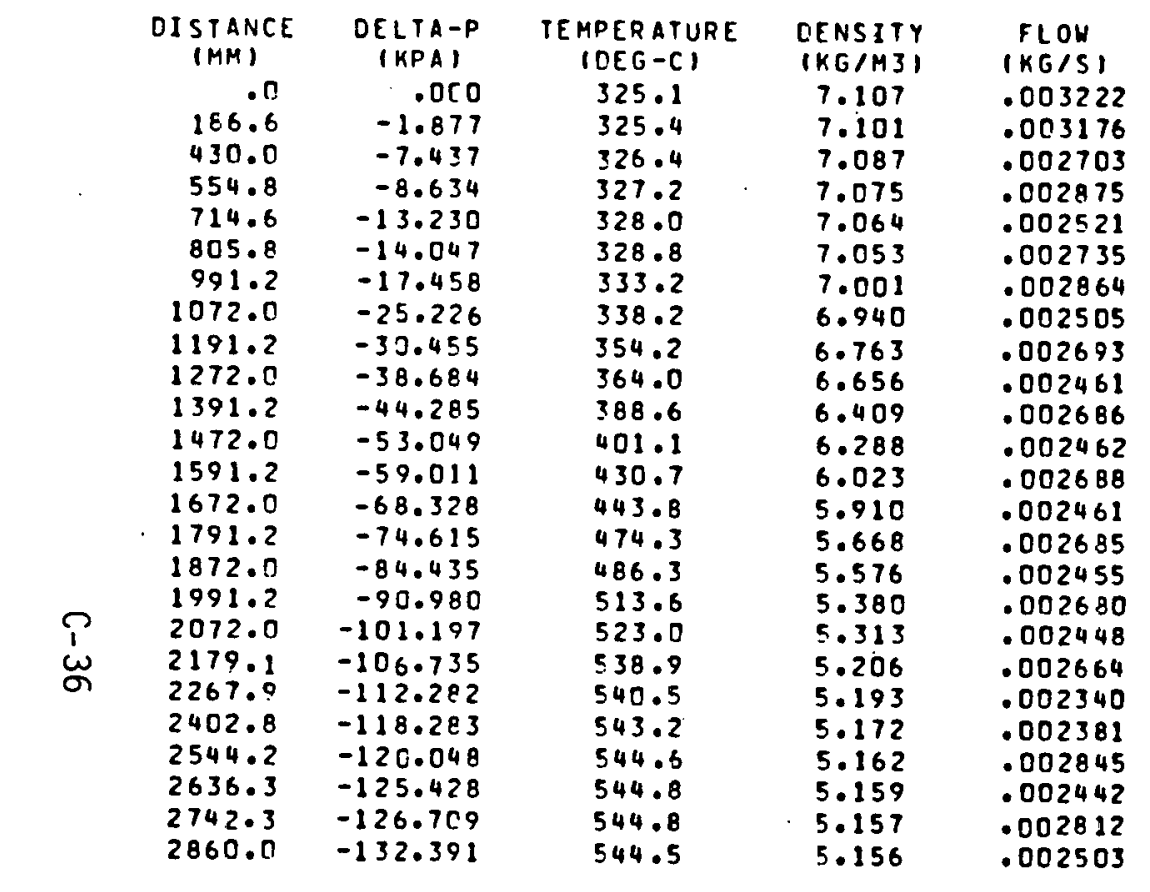

OATA FOR CHANNEL 25

$\begin{array}{ccc}\text { PEYNOLOS } & \text { AREA } & \text { FRICIION } \\ \text { NUMBER } & \text { IMMP) } & \text { FACTOR } \\ 80250 . & 11.69 & .01984 \\ 79080 . & 11.69 & .01990 \\ 67223 . & 11.69 & .02066 \\ 71441 . & 11.69 & .02037 \\ 62581 . & 11.69 & .02100 \\ 67839 . & 11.69 & .02061 \\ 71892 . & 12.13 & .05468 \\ 62531 . & 12.13 & .12434 \\ 66045 . & 12.13 & .12434 \\ 59710 . & 12.13 & .12434 \\ 63490 . & 12.13 & .12434 \\ 57454 . & 12.13 & .12434 \\ 60895 . & 12.13 & .12434 \\ 55043 . & 12.13 & .12434 \\ 58375 . & 12.13 & .12434 \\ 52785 . & 12.13 & .12434 \\ 56252 . & 12.13 & .12434 \\ 50956 . & 12.13 & .12434 \\ 53796 . & 11.69 & .09649 \\ 47178 . & 11.69 & .02244 \\ 47905 . & 11.69 & .02235 \\ 57174 . & 11.69 & .02144 \\ 490530 & 11.69 & .02223 \\ 56496 . & 11.69 & .02150 \\ 50306 . & 11.69 & .02210\end{array}$

DATE 27 MAY 8

TIM

$11: 21: 28$

\begin{tabular}{|c|c|c|c|c|}
\hline $\begin{array}{l}\text { ANTON } \\
\text { JMBER }\end{array}$ & $\begin{array}{l}F I L M H \\
K W / M Z^{-C}\end{array}$ & $\mathrm{H}+$ & $R(H+)$ & $A R$ \\
\hline .00301 & 4.303 & .0 & .00 & .0 \\
\hline .00302 & 4.256 & .0 & .00 & . \\
\hline .00313 & 3.759 & .0 & .00 & 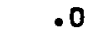 \\
\hline .00309 & 3.941 & .0 & .00 & 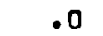 \\
\hline .00313 & 3.562 & .0 & .00 & 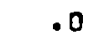 \\
\hline .00313 & 3.793 & .0 & .00 & $\cdot 0$ \\
\hline .00589 & 10.217 & 119.7 & 4.37 & 2. \\
\hline .00606 & 6.259 & 104.1 & 4.37 & \\
\hline .00599 & 9.771 & 110.0 & 4.37 & 2.1 \\
\hline .00612 & 6.209 & 99.4 & 4.37 & \\
\hline .00604 & 9.861 & 105.7 & 4.37 & 2 . \\
\hline .00617 & 6.253 & 95.7 & 4.37 & . \\
\hline .00610 & 9.993 & 101.4 & 4.37 & \\
\hline .00622 & 6.296 & 91.7 & 4.37 & 2.0 \\
\hline .00615 & 10.053 & 97.2 & 4.37 & 2. \\
\hline .00628 & 6.331 & 87.9 & 4.37 & 2. \\
\hline .00620 & 10.150 & 93.7 & 4.37 & 2. \\
\hline .00633 & 6.351 & 84.9 & 4.37 & 2. \\
\hline .00311 & 4.173 & 89.6 & 4.37 & 2.0 \\
\hline .00321 & 3.327 & .0 & .00 & .0 \\
\hline .00319 & 3.372 & .0 & .00 & $\bullet$ \\
\hline .00306 & 3.868 & .0 & .00 & .0 \\
\hline .00317 & 3.437 & .0 & .00 & \\
\hline .00307 & 3.832 & . 0 & .00 & . \\
\hline .00316 & 3.504 & .0 & .00 & 0 \\
\hline
\end{tabular}


CHANNEL RESULTS

CASE 1 CFTL BUNDLE - ONE SIXTH SECTION - FULLY ROUGH FLON - 1/13181

OATE 27 MAY Q1 TIME 11:21:28

TIME $=.00000$ SECONOS PRESSURE $=9000.0 \mathrm{KPA}$

\begin{tabular}{|c|c|c|c|c|}
\hline $\begin{array}{l}\text { (STANCE } \\
\text { (MM) }\end{array}$ & $\begin{array}{l}\text { DELTA } \\
\text { (KPA) }\end{array}$ & $\begin{array}{l}\text { TE MPER ATURE } \\
\text { (DEG -C) }\end{array}$ & $\begin{array}{l}\text { DENSITY } \\
\text { IKG/M3) }\end{array}$ & $\begin{array}{l}F L O W \\
|K G / S|\end{array}$ \\
\hline 186.0 & $\begin{array}{r}.000 \\
-1.877\end{array}$ & $\begin{array}{l}325.1 \\
325.4\end{array}$ & $\begin{array}{l}7.107 \\
7.101\end{array}$ & $\begin{array}{l}.003222 \\
.003176\end{array}$ \\
\hline 430.0 & -7.438 & 326.4 & 7.087 & .002712 \\
\hline 554.8 & -8.633 & 327.1 & 7.076 & .002868 \\
\hline 714.6 & -13.232 & 327.9 & 7.065 & .002521 \\
\hline 805.8 & -14.048 & 328.6 & 7.055 & .002709 \\
\hline 991.2 & -17.455 & 332.6 & $7.00^{7}$ & .002829 \\
\hline 1072.0 & -25.233 & 337.9 & 6.944 & .002501 \\
\hline 1191.2 & -30.446 & 352.0 & 6.786 & .002670 \\
\hline 1272.0 & -38.693 & 362.8 & 6.668 & .002472 \\
\hline 1391.2 & -44.274 & 384.1 & 6.452 & .002668 \\
\hline 1472.0 & -53.060 & 397.8 & 6.317 & .002474 \\
\hline 1591.2 & -58.999 & 423.5 & 6.084 & .002668 \\
\hline 1672.0 & -68.340 & 438.0 & 5.957 & .002472 \\
\hline 1791.2 & -74.602 & 464.8 & 5.740 & .002663 \\
\hline 1872.0 & -84.448 & 478.2 & 5.635 & .002464 \\
\hline 1991.2 & -90.966 & 502.9 & 5.454 & .002654 \\
\hline 2072.0 & -101.212 & 513.5 & 5.376 & .002453 \\
\hline 2179.1 & $-106 \cdot 718$ & 528.6 & 5.272 & .002626 \\
\hline 2267.9 & -112.286 & 530.7 & 5.255 & .002340 \\
\hline 2.402 .8 & -118.289 & 535.2 & 5.223 & .002291 \\
\hline 2544.2 & -120.043 & 538.4 & 5.200 & .002672 \\
\hline $2636 \cdot 3$ & -125.433 & 538.9 & 5.195 & .002331 \\
\hline 2742.3 & -126.705 & 539.8 & 5.183 & .002628 \\
\hline 2860.0 & -132.391 & 540.2 & 5.183 & .002349 \\
\hline
\end{tabular}

DATA FOP CHANNEL 26

$\begin{array}{ccc}\text { PEYNOLDS } & \text { AREA } & \text { FRICIION } \\ \text { NUMBER } & \text { IMM2 I } & \text { FACTOR } \\ 80250 . & 11.69 & .01984 \\ 79079 . & 11.69 & .01990 \\ 67446 . & 11.69 & .02064 \\ 71261 . & 11.69 & .02038 \\ 62594 . & 11.69 & .02100 \\ 67198 . & 11.69 & .02066 \\ 71057 . & 12.13 & .05472 \\ 62431 . & 12.13 & .12434 \\ 65519 . & 12.13 & .12434 \\ 60038 . & 12.13 & .12434 \\ 63358 . & 12.13 & .12434 \\ 57917 . & 12.13 & .12434 \\ 60879 . & 12.13 & .12434 \\ 55802 . & 12.13 & .12434 \\ 58408 . & 12.13 & .12434 \\ 53387 . & 12.13 & .12434 \\ 56230 . & 12.13 & .12434 \\ 51484 . & 12.13 & .12434 \\ 53490 . & 11.69 & .09650 \\ 47574 . & 11.69 & .02239 \\ 46398 . & 11.69 & .02252 \\ 53963 . & 11.69 & .02174 \\ 47071 . & 11.69 & .02245 \\ 53626 . & 11.69 & .02183 \\ 47378 . & 11.69 & .02241\end{array}$

STANTON
NUMBER
.00301
.00302
.00313
.00309
.00318
.00313
.00590
.00606
.00600
.00611
.00604
.00616
.00610
.00621
.00615
.00626
.00620
.00631
.00312
.00321
.00322
.00311
.00321
.00312
.00320

a

$R(H+)$

FIL $M / M 2-C$

4.303

4.256

3.768

3.933

3.562

3.764

10.127

6.249

9.705

6.228

9.803

6.273

9.923

6.313

9.972

6.342

10.055

6.352

.128

3.328

3.274

3.685

3.318

3.639
3.337
H+

.0

.0

- D

.0

118.3

104.0

109.3

100.0

105.5

96.5

101.4

92.6

97.3

88.9

93.7

85.7

89.1

1.1

.0

.0
.0
.0

.0
$.00 \quad .0$

$.00 \quad .0$

$.00 \quad .0$

$.00 \quad .0$

$\begin{array}{rr}.00 & .0 \\ 4.37 & 2.0\end{array}$

4.37 .2 .0

$4.37 \quad 2.0$

$4.37 \quad 2.0$

$4.37 \quad 2.0$

$\begin{array}{ll}4.37 & 2.0\end{array}$

$4.37 \quad 2.0$

$4.37 \quad 2.0$

$4.37 \quad 2.0$

$4.37 \quad 2.0$

4.372 .0

$.00 \quad .0$

.00

.00

.00

.00
.00 
CHANNEL RESULTS

IIME $=.00000$ SECONOS PRESSURE $=9000.0 \mathrm{KPA}$

TEMPERATURE OENSITY

\begin{tabular}{|c|c|c|c|c|}
\hline $\begin{array}{l}\text { ISTANCE } \\
\text { (MM) }\end{array}$ & $\begin{array}{l}\text { OELTA-P } \\
\text { (KPA) }\end{array}$ & $\begin{array}{l}\text { TE MPERATURE } \\
\text { (OEG-C) }\end{array}$ & $\begin{array}{l}\text { DENSITY } \\
\text { (KG/MS) }\end{array}$ & $\begin{array}{l}F(O N) \\
(K G / S)\end{array}$ \\
\hline $\begin{array}{r}.0 \\
186.6\end{array}$ & $\begin{array}{r}.000 \\
-1.877\end{array}$ & $\begin{array}{l}325.1 \\
325.6\end{array}$ & $\begin{array}{l}7.107 \\
7.100\end{array}$ & $\begin{array}{l}.001610 \\
.001589\end{array}$ \\
\hline 430.0 & -7.439 & 326.6 & 7.084 & .001388 \\
\hline 554.8 & -8.633 & 327.3 & 7.074 & .001459 \\
\hline 714.6 & -13.233 & 328.2 & 7.061 & .001299 \\
\hline 805.8 & -14.048 & 328.8 & 7.053 & .001384 \\
\hline 991.2 & -17.454 & 333.1 & 7.002 & .001424 \\
\hline 1072.0 & -25.236 & 339.5 & 6.927 & .001276 \\
\hline 1191.2 & -30.441 & 353.6 & 6.769 & .001344 \\
\hline 1272.0 & -38.699 & 365.6 & 6.640 & .001264 \\
\hline 1391.2 & -44.269 & 385.4 & 6.439 & .001341 \\
\hline 1472.0 & -53.067 & 399.8 & 6.299 & .001263 \\
\hline 1591.2 & -58.993 & 423.3 & 6.086 & .001339 \\
\hline 1672.0 & -69.348 & 438.3 & 5.955 & .001261 \\
\hline 1791.2 & -74.595 & 462.7 & 5.756 & .001334 \\
\hline $\begin{array}{r}1872.0 \\
1991.2\end{array}$ & $\begin{array}{l}-84.457 \\
-90.959\end{array}$ & $\begin{array}{l}476.4 \\
499.0\end{array}$ & 5.648 & .001255 \\
\hline $\begin{array}{l}1991.2 \\
2072.0\end{array}$ & -101.221 & 509.7 & $\begin{array}{l}3.481 \\
5.402\end{array}$ & $\begin{array}{l}.001327 \\
.001247\end{array}$ \\
\hline 2170.1 & -106.708 & 523.5 & 5.305 & .001308 \\
\hline 2267.9 & -112.289 & $525 \cdot 3$ & 5.290 & .001175 \\
\hline 2402.8 & -118.292 & 529.6 & 5.259 & .001103 \\
\hline 2544.2 & -120.041 & 533.7 & 5.231 & .001256 \\
\hline 2636.3 & -125.437 & 534.2 & 5.225 & .001119 \\
\hline 2742.3 & -126.705 & 535.8 & 5.213 & .001234 \\
\hline 360.0 & -132.391 & 536.6 & 5.206 & .001104 \\
\hline
\end{tabular}

DATA FOR CHANNEL 27

$\begin{array}{ccc}\text { REYNOLOS } & \text { AREA } & \text { FRICTION } \\ \text { NUMBER } & \text { IMM2 } & \text { FACIOR } \\ 80183 . & 5.84 & .01984 \\ 79123 . & 5.84 & .01990 \\ 69009 . & 5.84 & .02053 \\ 72505 . & 5.84 & .02030 \\ 64490 . & 5.84 & .02085 \\ 68665 . & 5.84 & .02056 \\ 71482 . & 6.06 & .05470 \\ 63597 . & 6.06 & .12434 \\ 65939 . & 6.06 & .12434 \\ 61221 . & 6.06 & .12434 \\ 63628 . & 6.06 & .12434 \\ 59039 . & 6.06 & .12434 \\ 61123 . & 6.06 & .12434 \\ 56706 . & 6.06 & .12434 \\ 58653 . & 6.06 & .12434 \\ 54476 . & 6.06 & .12434 \\ 5644 . & 6.06 & .12434 \\ 52519 . & 6.06 & .12434 \\ 53534 . & 5.84 & .09650 \\ 4800 B . & 5.84 & .02234 \\ 44893 . & 5.84 & .02270 \\ 50965 . & 5.84 & .02203 \\ 45359 . & 5.84 & .02265 \\ 49979 . & 5.84 & .02213 \\ 44685 . & 5.84 & .02273\end{array}$

$\begin{array}{lr}\text { STANTON } & \text { FILMH } \\ \text { NUMBER } & \text { KHIM2-C } \\ .00301 & 4.303 \\ .00302 & 4.261 \\ .00311 & 3.838 \\ .00308 & 3.990 \\ .00316 & 3.648 \\ .00312 & 3.831 \\ .00589 & 10.183 \\ .00604 & 6.356 \\ .00599 & 9.162 \\ .00609 & 6.347 \\ .00604 & 9.853 \\ .00613 & 6.386 \\ .00609 & 9.957 \\ .00619 & 6.419 \\ .00614 & 9.990 \\ .00624 & 6.440 \\ .00619 & 10.057 \\ .00629 & 6.438 \\ .00313 & 4.120 \\ .00320 & 3.343 \\ .00325 & 3.183 \\ .00315 & 3.518 \\ .00324 & 3.217 \\ .00317 & 3.470 \\ .00325 & 3.185\end{array}$

(⿸丆口

911901

$R(H \bullet)$ 
DIVERSION CROSSFLOW BETHEEN ADJACENT CHANERLS, HII,J), (KG/SEC-M).
CASE 1
CFTL BUNCLE - ONE SIXTH SECTION - FULLY ROUGH FLON - 1/13/81
DATE 27 MAY O 1
T I HE $11: 21: 28$

TIME $=.00000$ SECONOS

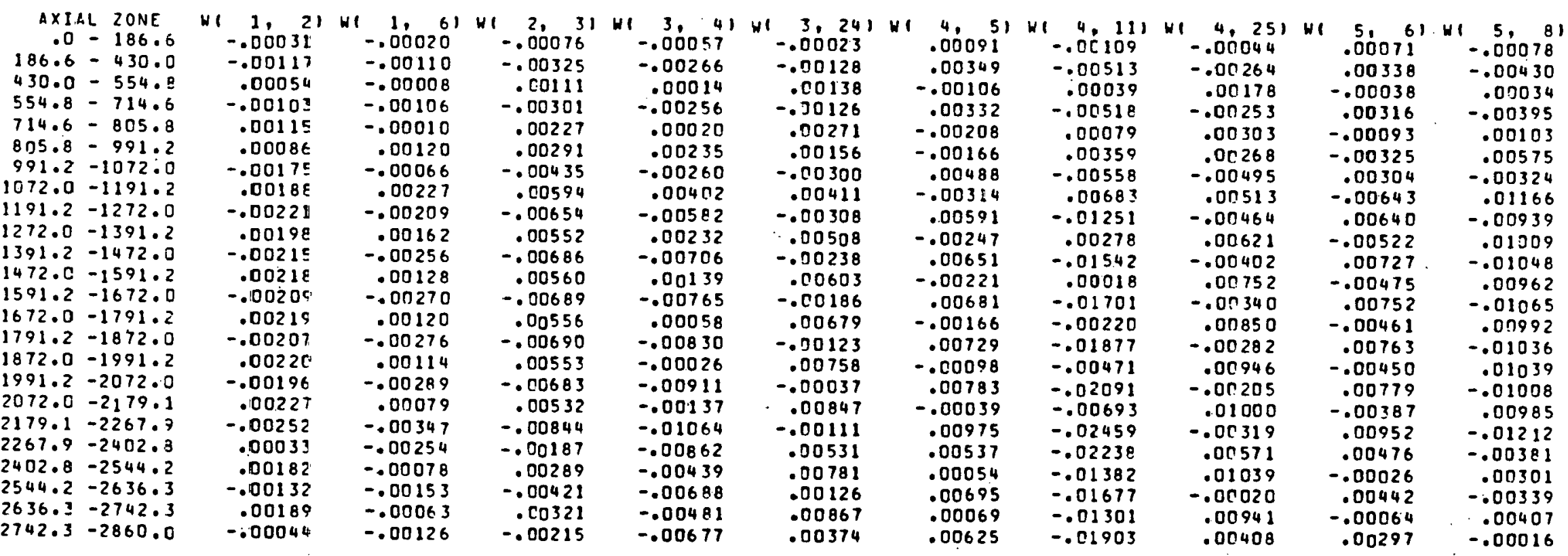


DIVERSION CROSSFLOW BETHEEN AOJACENT CHANNELS, WII,JI, (KG/SFC-M).

CASE 1 CFTL EUNDLE - ONE SIXTH SECTION - FULLY POUGH FLOH - 1/13/81 TIME 11:21:28 TIME $=.00000$ SECONDS

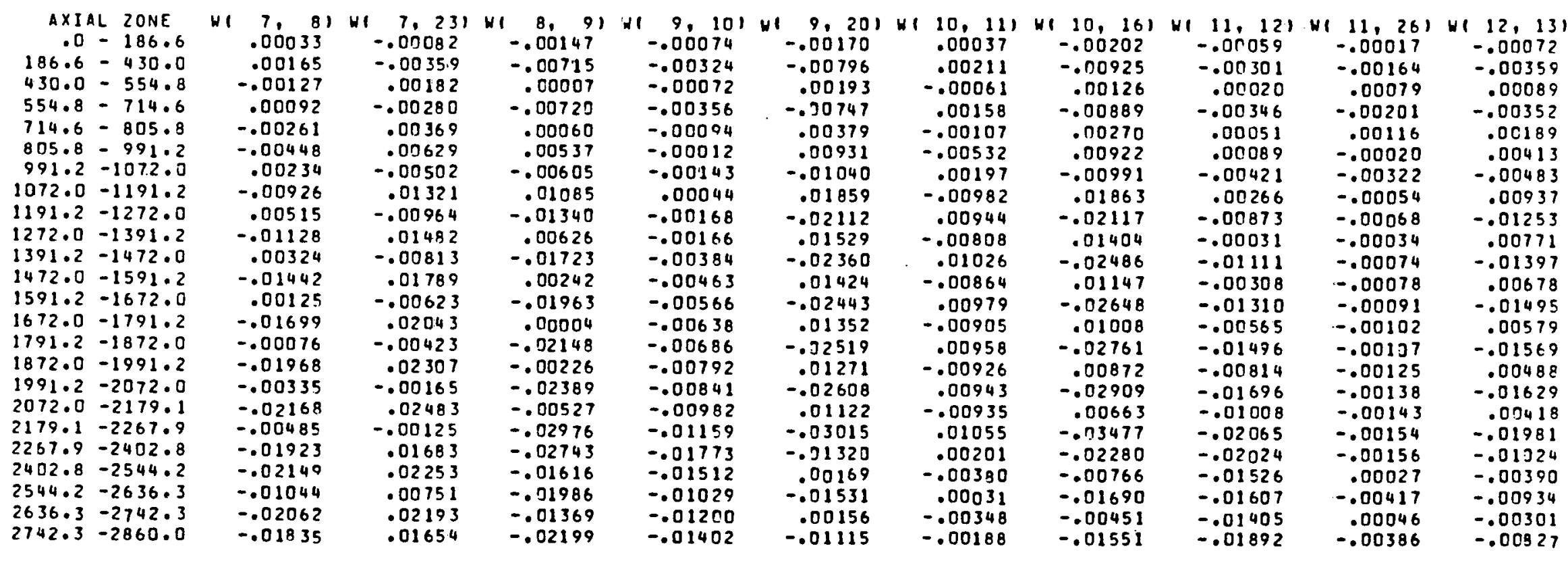


OIVERSION CROSSFLON BETHEEN AJJACENT CHANNELS, WII,JI, IKG/SEC-M).

CASE 1 CFTL BUNDLE - ONE SIXTH SECTION - FULLY ROUGH FLOH - 1/13/81 TIME 11:21:28

TIME $=.00000$ SECONOS

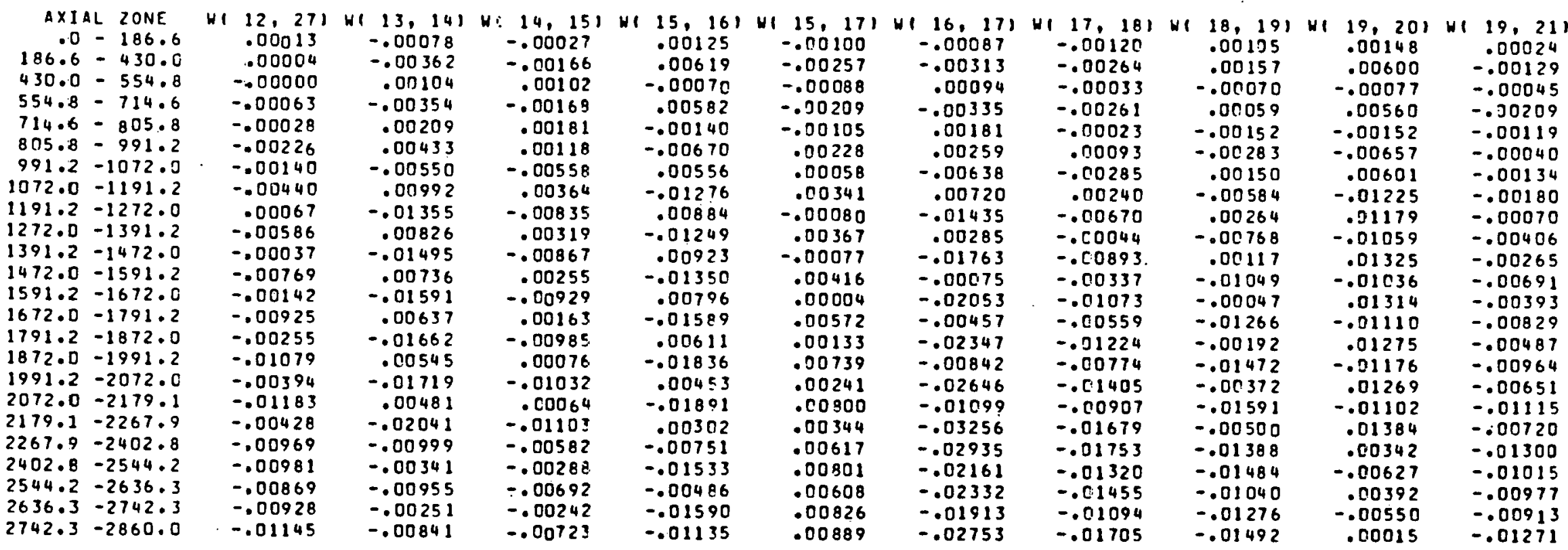


DIVERSION CROSSFLON BETHEEN AOJACENT CHANNELS, HIJ,J), (KG/SEC-H).

CASE 1 CFTL BUNDLE - ONE SIXTH SECTION - FULLY ROUEH FLON - 1/13/81 TIME 11:21:28 TIME $=.00000$ SECONDS

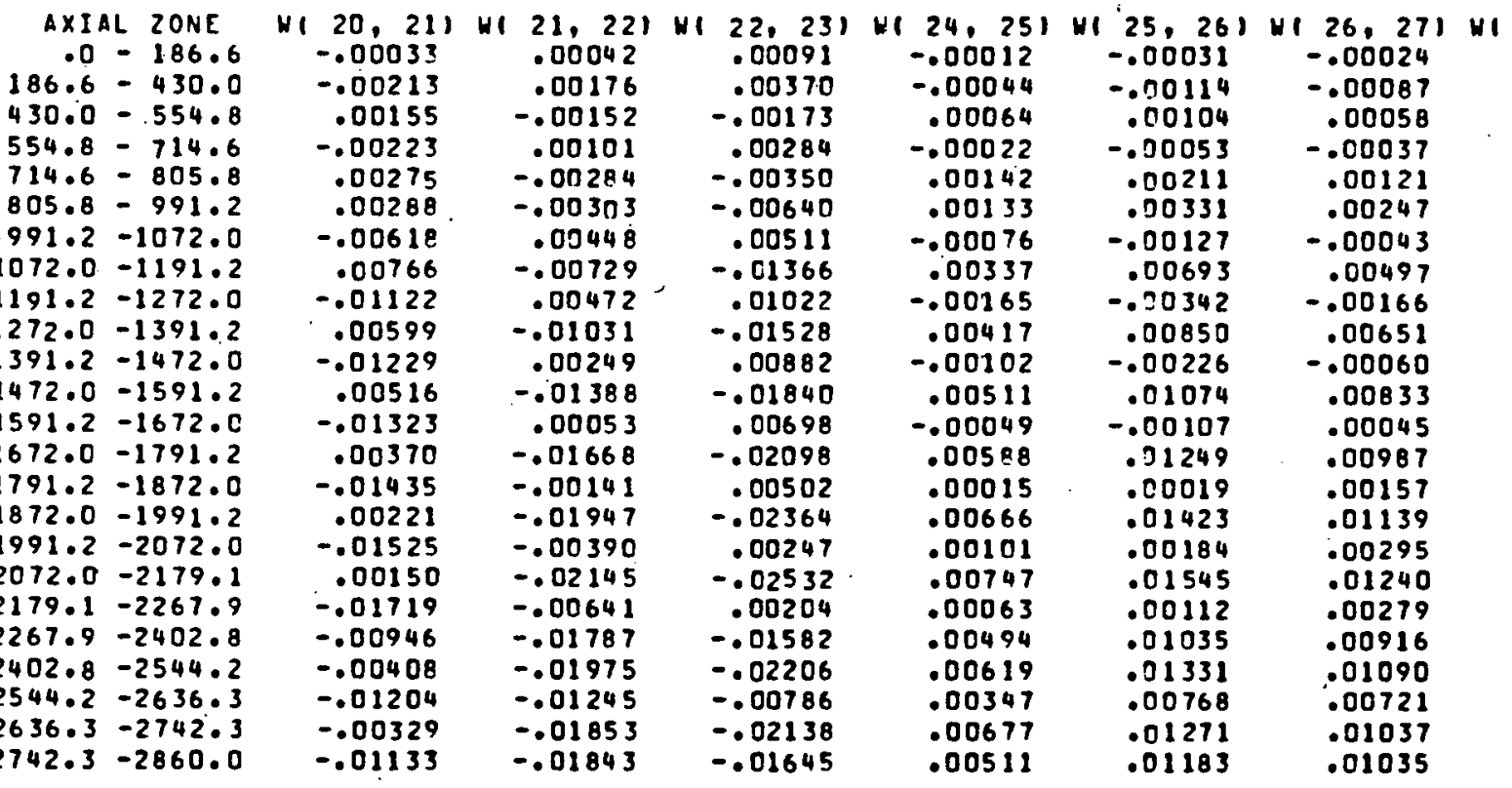




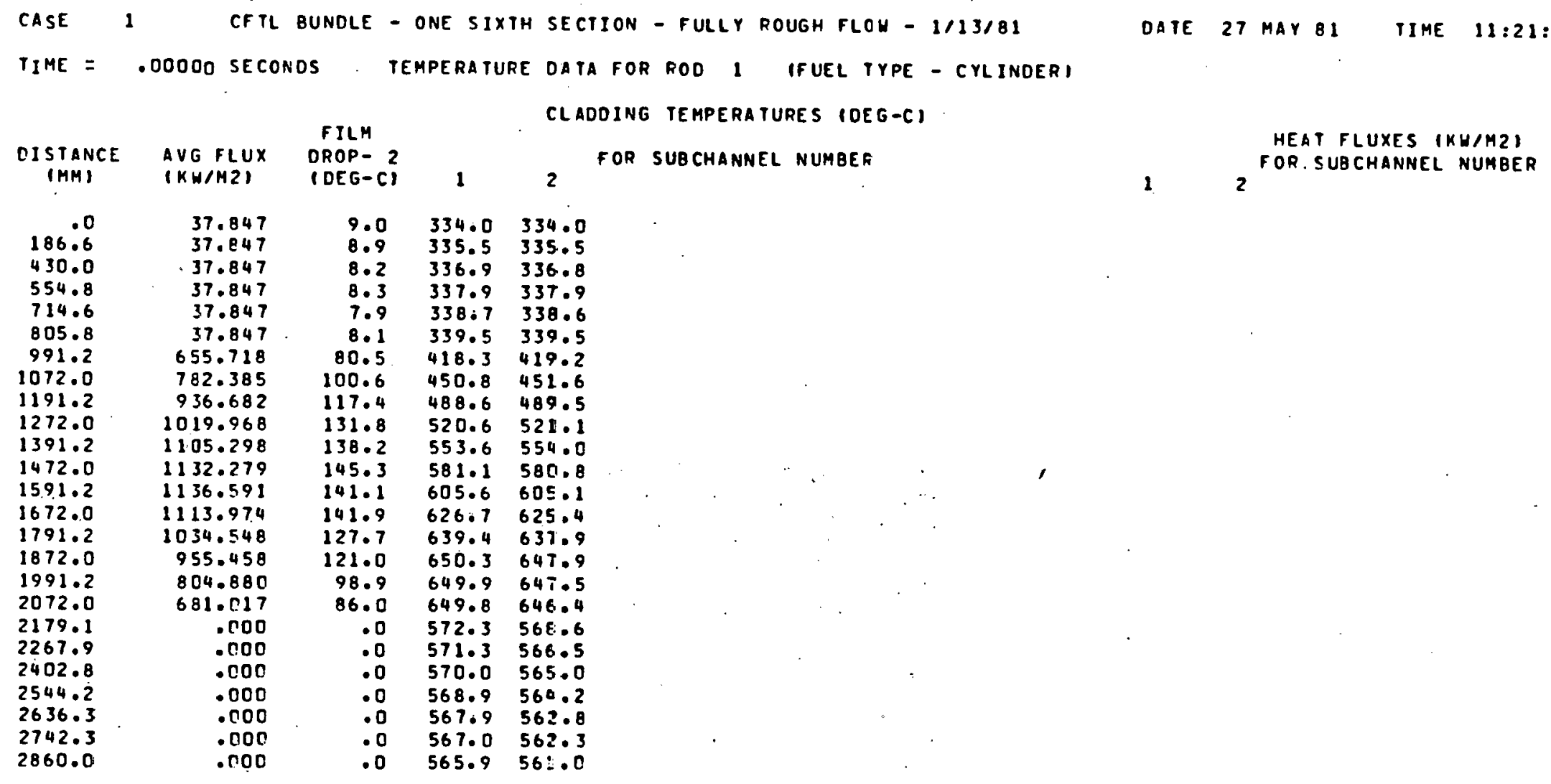


CASE 1 CFTL BUNDLE - ONE SIXTH SECTION - FULLY POUGH FLON - 1/13/81

TIME $=.00000$ SECONDS TEMPERA TURE DA

CLADOING TEMPERATURES (OEG-C)
FILM

DISTANCE

(MM)

AVG FLUX

430.0

554.8

714.6

805.8

991.2

1072.0

1191.2

1272.0

1391.2

1472.0

1591.2

1672.0

1791.2

1872.0

i 1991.2

2072.0

2179.1

2267.9

2402.8

$2544 \cdot 2$

2636.3

2742.3

2860.0
37.847
37.847

37.847

(DEG-C)

37.847

37.847

37.847

655.718

782.385

936.682

1019.968

1105.298

1132.279

1136.591

1113.974

1034.548

955.458

804.880

681.017

.000
.000

.000

. 000

.000

.000

.

$\begin{array}{rllllll}9.0 & 334.0 & 334.0 & 333.8 & 333.8 & 334.0 & 334.0 \\ 8.8 & 335.5 & 335.5 & 335.3 & 335.4 & 335.5 & 335.5 \\ 8.2 & 336.9 & 336.8 & 336.7 & 336.8 & 336.9 & 336.9 \\ 8.2 & 337.9 & 337.9 & 337.8 & 337.9 & 337.9 & 337.9 \\ 7.8 & 338.7 & 338.6 & 338.5 & 338.5 & 338.6 & 338.7 \\ 8.0 & 339.5 & 339.5 & 339.4 & 339.4 & 339.5 & 339.6 \\ 79.4 & 418.3 & 419.0 & 421.0 & 421.2 & 418.7 & 418.2 \\ 99.6 & 450.8 & 451.6 & 453.8 & 453.9 & 451.2 & 450.8 \\ 116.2 & 488.6 & 489.5 & 491.8 & 492.6 & 489.2 & 488.5 \\ 130.5 & 520.6 & 521.1 & 522.3 & 522.7 & 520.8 & 520.6 \\ 137.0 & 553.6 & 554.0 & 554.7 & 555.9 & 554.0 & 553.7 \\ 144.0 & 581.1 & 580.8 & 580.3 & 580.3 & 580.8 & 581.1 \\ 139.9 & 605.6 & 605.1 & 603.9 & 604.3 & 605.2 & 605.6 \\ 140.7 & 626.7 & 625.4 & 622.9 & 621.6 & 625.3 & 626.7 \\ 126.7 & 639.4 & 637.9 & 634.7 & 633.5 & 637.7 & 639.4 \\ 120.0 & 650.3 & 647.9 & 643.3 & 640.2 & 647.3 & 650.1 \\ 98.1 & 649.9 & 647.5 & 642.2 & 638.8 & 646.6 & 649.7 \\ 85.3 & 649.8 & 646.4 & 639.8 & 634.7 & 644.8 & 649.2 \\ .0 & 572.3 & 568.6 & 560.0 & 554.3 & 568.9 & 571.8 \\ .0 & 571.3 & 566.5 & 556.5 & 549.2 & 564.2 & 570.2 \\ .0 & 570.0 & 565.0 & 553.6 & 545.1 & 561.3 & 568.4 \\ .0 & 568.9 & 564.2 & 553.2 & 543.8 & 559.9 & 567.4 \\ .0 & 567.9 & 562.8 & 551.8 & 542.7 & 557.9 & 566.0 \\ .0 & 567.0 & 562.3 & 551.6 & 542.3 & 557.0 & 565.2 \\ .0 & 565.9 & 561.0 & 550.1 & 541.3 & 555.1 & 563.7\end{array}$

DATE 27 MAY 81 TIME 11:21:28
HEAT FLUXES (KW/HZ)

1 
CASE 1 CFTL BUNOLE - ONE SIXTH SECTION - FULLY ROUGH FLOW - 1/13/81

TIME $=.00000$ SECONDS

TEMPERA TURE DATA FOR ROD 3 IFUEL TYPE - CYLINDERI

CLADDING TEMPERATURES (OEG-C)

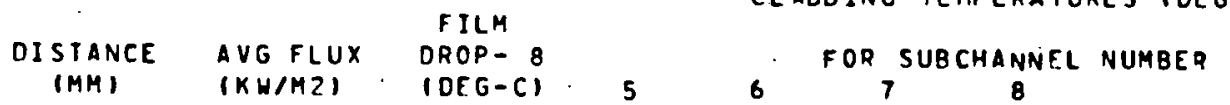

$\begin{array}{rr}.0 & 37.847 \\ 186.6 & 37.847 \\ 430.0 & 37.847 \\ 554.8 & 37.847 \\ 714.6 & 37.847 \\ 805.8 & 37.847 \\ 991.2 & 655.718 \\ 1072.0 & 782.385 \\ 1191.2 & 936.682 \\ 1272.0 & 1019.968 \\ 1391.2 & 1105.298 \\ 1472.0 & 1132.279 \\ 1591.2 & 1136.591 \\ 1672.0 & 1113.974 \\ 1791.2 & 1034.548 \\ 1872.0 & 955.458 \\ 1991.2 & 804.880 \\ 2072.0 & 681.017 \\ 2179.1 & .000 \\ 2267.9 & .000 \\ 2402.8 & .000 \\ 2544.2 & .000 \\ 2636.3 & .000 \\ 2742.3 & .000 \\ 2860.0 & .000\end{array}$

$\begin{array}{rllll}9.0 & 334.0 & 334.0 & 334.0 & 334.0 \\ 8.8 & 335.5 & 335.5 & 335.4 & 335.5 \\ 8.2 & 336.9 & 336.9 & 336.8 & 336.8 \\ 8.3 & 337.9 & 337.9 & 337.8 & 337.9 \\ 7.8 & 338.6 & 338.7 & 338.5 & 338.6 \\ 8.0 & 339.5 & 339.6 & 339.4 & 339.5 \\ 79.7 & 418.7 & 418.2 & 419.0 & 418.4 \\ 99.6 & 451.2 & 450.8 & 451.1 & 450.7 \\ 116.4 & 489.1 & 488.5 & 488.5 & 488.5 \\ 130.4 & 520.8 & 520.6 & 518.6 & 519.7 \\ 137.1 & 554.0 & 553.7 & 551.2 & 552.8 \\ 143.8 & 580.7 & 581.1 & 576.0 & 578.8 \\ 139.9 & 605.2 & 605.6 & 600.3 & 603.1 \\ 140.4 & 625.2 & 626.7 & 618.6 & 622.2 \\ 126.6 & 637.7 & 639.4 & 631.5 & 634.6 \\ 119.6 & 647.3 & 650.1 & 640.0 & 643.1 \\ 98.0 & 646.6 & 649.7 & 639.9 & 642.4 \\ 85.0 & 644.8 & 649.2 & 638.2 & 639.6 \\ .0 & 566.9 & 571.8 & 560.9 & 562.1 \\ .0 & 564.1 & 570.2 & 559.4 & 558.1 \\ .0 & 561.3 & 568.4 & 556.8 & 553.7 \\ .0 & 559.9 & 567.4 & 554.5 & 551.6 \\ .0 & 557.9 & 566.0 & 553.3 & 549.7 \\ .0 & 557.0 & 565.2 & 551.7 & 548.7 \\ .0 & 555.1 & 563.7 & 550.0 & 546.6\end{array}$

DATE 27 MAY 81 TIME $11.21: 28$

HEAT FLUXES $|K H / M 2|$ 
CASE 1

CFTL BUNDLE - ONE SIXTH SECIION - FULLY ROUGH FLOH - 1113181

TIME $=.00000$ SECONOS

TEMPERATURE DATA FOR ROD

DISTANCE AVG FLUX DILP

(HM)

(KH/MZ)

IDEG-CI

24

$\begin{array}{ll}186.0 & .000 \\ 186.6 & .000 \\ 430.0 & .000 \\ 554.8 & .000 \\ 714.6 & .000 \\ 805.8 & .000 \\ 991.2 & .000 \\ 1072.0 & .000 \\ 1191.2 & .000 \\ 1272.0 & .000 \\ 1391.2 & .000 \\ 1472.0 & .000 \\ 1591.2 & .000 \\ 1672.0 & .000 \\ 1791.2 & .000 \\ 1872.0 & .000 \\ 1991.2 & .000 \\ 2072.0 & .000 \\ 2179.1 & .000 \\ 2267.9 & .000 \\ 2402.8 & .000 \\ 2544.2 & .000 \\ 2636.3 & .000 \\ 2742.3 & .000 \\ 2860.0 & .000\end{array}$

IFUEL TYPE - CYLINDER

CLADDING TEMPERATURES (DEG-C)

FOR SUBCHANNEL NUMBEP

DATE

27 MAY $B$

TIME 11:21:28

HEAT FLUXES IKH/HZ)

$25 \quad 26 \quad 27$

24

26

\section{NEL NUMBER}


CASE. I

CF TL BUNOLE - ONE SIXTH SECTION - FULLY ROUGH FLON - 1/13181

DATE 27 MAY B.

TIME $11: 21: 28$

TIME $=.00000$ SECONDS

TEMPERATURE DATA FOR ROD

IF UEL TYPE - CYLINDERI

$\begin{array}{lll}\text { OISTANCE } & \text { AVGFLUX } & \text { FILM } \\ \text { (MA) } & \text { (KHIMZI) } & \text { (OEG-C) }\end{array}$

CLADDING TEMPERATURES IOEG-CI

$\begin{array}{rr}100 & 37.847 \\ 186.6 & 37.847 \\ 430.0 & 37.847 \\ 554.8 & 37.847 \\ 714.6 & 37.847 \\ 805.8 & 37.847 \\ 991.2 & 655.718 \\ 1072.0 & 782.385 \\ 1191.2 & 936.682 \\ 1272.0 & 1019.968 \\ 1391.2 & 1105.298 \\ 1472.0 & 1132.279 \\ 1591.2 & 1136.591 \\ 1672.0 & 1113.974 \\ 1791.2 & 1034.548 \\ 1872.0 & 955.458 \\ 1991.2 & 804.880 \\ 2072.0 & 681.017 \\ 2179.1 & .000 \\ 2267.5 & .500 \\ 2402.8 & .000 \\ 2544.2 & .000 \\ 2636.3 & .000 \\ 2742.3 & .000 \\ 2860.0 & .000\end{array}$

\section{$\begin{array}{ccc}\text { FOR SUBCHANNEL NUMBER } \\ 8 & 9 & 10\end{array}$}

11

8.8
8.8

333.9

9

10

$8.4 \quad 336.8 \quad 336.9 \quad 336.8335 .535 .535$.

$8.5 \quad 337.8 \quad 337.9 \quad 337.0 \quad 336.8336 .7 \quad 335.8$

$8.2 \quad 338.5 \quad 33 . .9 \quad 337.9 \quad 337.8 \quad 337.8 \quad 337.8$

$\begin{array}{llllll}338.5 & 338.6 & 338.6 & 338.5 & 338.4 & 338.5\end{array}$

$\begin{array}{llllll}339.4 & 330.5 & 339.5 & 339.0 & 339.3 & 339.4\end{array}$

$\begin{array}{llllll}421.2 & 418.7 & 418.4 & 419.0 & 419.8 & 421.9\end{array}$

$453.9 \quad 45: .2 \quad 450.7 \quad 451.0 \quad 451.7 \quad 454.2$

$\begin{array}{llllll}492.6 & 489.2 & 488.5 & 488.7 & 489.7 & 493.0\end{array}$

$\begin{array}{lllllll}522.7 & 520.8 & 519.7 & 518.7 & 519.2 & 522.1\end{array}$

$\begin{array}{lllllll}142.9 & 555.8 & 554.0 & 552.8 & 551.3 & 551.8 & 554.7\end{array}$

$\begin{array}{lllllll}48.4 & 580.2 & 580.8 & 578.8 & 575.2 & 574.9 & 577.3\end{array}$

$\begin{array}{lllllll}145.5 & 604.3 & 605.2 & 603.1 & 598.9 & 598.2 & 599.9\end{array}$

$\begin{array}{lllllll}144.8 & 621.6 & 625.3 & 622.2 & 615.4 & 613.5 & 615.2\end{array}$

$\begin{array}{lllllll}121.6 & 633.5 & 637.7 & 634.6 & 627.1 & 624.6 & 625.4\end{array}$

$1 \bar{c} 3.3 \quad 640.2647 .3 \quad 643.1632 .9629 .1630 .3$

$101.8 \quad 638.8 \quad 645.6 \quad 642.44631 .7627 .2 \quad 627.6$

$87.6 \quad 634.6 \quad 644.8 \quad 639.6 \quad 6260.027 .0627 .6$

$87.6 \quad 634.6 \quad 644.8 \quad 639.6 \quad 626.8621 .0622 .1$

554.3566 .9

$\begin{array}{llllll}549.2 & 564.2 & 558.1 & 544.3 & 536.8 & 536.2\end{array}$

$\begin{array}{lllllll}545.1 & 561 . ? & 553.7 & 540.6 & 532.4 & 532.7\end{array}$

$543.8 \quad 559.9 \quad 551.6 \quad 539.1 \quad 531.0 \quad 532.0$

$\begin{array}{lllllll}542.7 & 557.9 & 549.7 & 537.9 & 529.6 & 531.7\end{array}$

$542.3 \quad 557.0 \quad 548.7 \quad 537.1 \quad 529.3 \quad 531.0$

$541.3553 .1 \quad 546.6 \quad 536.3528 .5 \quad 532.0$
HEAT F.LUXES (KH/MZ)

FOR SUSCHANNEL NUMBER

5 
CASE 1 CFTL BUNCLE - ONE SIXIH SECTION - FULLY ROUGH FLOH - 1/13/81

TIME $=.00000$ SECONDS
TEMPERATURE OATA FOR ROD 6 (FUEL TYPE - CYLINDERI

CLADDING TEMPERATURES (DEG-C)

$\begin{array}{cr}\text { DISTANCE } & \text { AVG FLUX } \\ \text { (MM) } & \begin{array}{r}\text { (KW/M2) } \\ .0\end{array} \\ 186.6 & 37.847 \\ 430.0 & 37.847 \\ 554.8 & 37.847 \\ 714.6 & 37.847 \\ 805.8 & 37.847 \\ 991.2 & 37.847 \\ 1072.0 & 755.718 \\ 1191.2 & 932.385 \\ 1272.0 & 1019.9868 \\ 1391.2 & 1105.298 \\ 1472.0 & 1132.279 \\ 1591.2 & 1136.591 \\ 1672.0 & 1113.974 \\ 1791.2 & 1034.548 \\ 1872.0 & 955.458 \\ 1991.2 & 804.880 \\ 2072.0 & 681.017 \\ 2179.1 & .000 \\ 2267.9 & .000 \\ 2402.8 & .000 \\ 2544.2 & .000 \\ 2636.3 & .000 \\ 2742.3 & .000 \\ 2860.0 & .000 \\ & \end{array}$

FILM

OROP -23

IDEG-CI

9.0

8.9

7.5

8.6
7.2

8.2

76.9

101.5

110.3

131.2
129.0

129.0
144.0

131.5

140.4

119.1

119.7

92.3
85.1

85.1

.0

.0

.0
.0
.0
.0

\section{FOR SUBCHANNEL NUMBER}

8 FOR SUBCHANNEL NUMBER

22

23

$\begin{array}{lllllll}35.0 & 335.5 & 335.3 & 335.9 & 335.9 & 335.3\end{array}$

$\begin{array}{lllllll}337.8 & 337.9 & 337.8 & 337.8 & 339.3 & 337.6 & 337.5\end{array}$

$\begin{array}{lllllll}338.5 & 338.6 & 338.5 & 338.5 & 341.7 & 336.1 & 337.1\end{array}$

$\begin{array}{lllllll}339.4 & 339.5 & 339.3 & 339.5 & 341.6 & 338.9 & 338.9\end{array}$

$\begin{array}{lllllll}419.0 & 418.4 & 419.0 & 422.2 & 405.6 & 419.5 & 414.8\end{array}$

$\begin{array}{lllllll}451.0 & 450.7 & 451.0 & 453.6 & 495.3 & 462.4 & 450.2\end{array}$

$\begin{array}{lllllll}488.5 & 488.5 & 488.7 & 487.9 & 454.7 & 470.5 & 479.5\end{array}$

$\begin{array}{lllllll}518.6 & 519.7 & 518.7 & 516.2 & 561.2 & 518.6 & 512.5\end{array}$

$\begin{array}{llllllll}551.2 & 552.8 & 551.3 & 545.3 & 501.2 & 519.5 & 537.3\end{array}$

$\begin{array}{lllllll}576.0 & 578.8 & 575.2 & 567.1 & 612.6 & 566.4 & 565.5\end{array}$

600.3603 .1598 .9588 .4539 .4559 .8503 .9

$618.6 \quad 622.2615 .4602 .7644 .4601 .8505 .9$

$618.6 \quad 622.2615 .4 \quad 602.7644 .4 \quad 601.2605 .6$

$\begin{array}{lllllll}640.0 & 643.1 & 632.9 & 617.6 & 651.3 & 617.2 & 626.5\end{array}$

$\begin{array}{lllllll}639.9 & 642.4 & 631.7 & 617.1 & 578.1 & 599.1 & 625.5\end{array}$

$\begin{array}{lllllll}638.2 & 639.6 & 626.8 & 611.2 & 633.5 & 613.3 & 626.5\end{array}$

$\begin{array}{lllllll}560.9 & 562.1 & 549.2 & 535.3 & 523.9 & 532.9 & 553.9\end{array}$

$\begin{array}{llllllll}559.4 & 558.1 & 544.3 & 530.2 & 525.2 & 533.0 & 550.8\end{array}$

$554.5 \quad 551.6 \quad 539.1532 .6 \quad 534.9 \quad 543.6552 .8$

$553.3549 .7 \quad 539.1 \quad 532.6 \quad 534.9 \quad 543.6 \quad 552.8$

$\begin{array}{lllllll}553.3 & 549.7 & 537.9 & 533.6 & 536.5 & 544.5 & 551.9\end{array}$

$\begin{array}{lllllll}551.7 & 548.7 & 537.1 & 534.8 & 539.1 & 548.2 & 551.2 \\ 550.0 & 546.6 & 536.3 & 536.7 & 541.1 & 547.0 & 550.1\end{array}$
$631.4 \quad 634.6 \quad 627.1 \quad 613.5 \quad 566.5 \quad 588.0 \quad 615.0$

$\begin{array}{lllllll}556.8 & 553.7 & 540.6 & 530.4 & 529.8 & 538.8 & 552.7\end{array}$
DATE 27 MAY 81 TIME 11:21:28

HEAT FLUXES (KH/MZ)

FOR SUBCHANNEL NUMBER

23 
CASE 1

CFTL BUNDLE - ONE SIXTH SECTION - FULLY ROUGH FLON - $1 / 13181$

OATE 27 MAY 81

TIME $11: 21: 28$

TIME = .0ODOO SECONOS TEMPERATURE DATA FOR ROD 7 IFUEL TYPE - CYLINOERI

CLADOING TEMPERATURES (DEG-C)

FILM

DISTANCE AVGFLUX OROP-20 (KW/MZ)

OROP- 20
|DEG-CI

37.847
37.847
37.847
37.847
37.847
37.847
655.718
782.385
936.582
1019.968
1105.298
1132.279
1136.591
1113.974
1034.548
955.458
804.880
681.017
.000
.000
.000
.000
.000
.000
.000

FOR SUBCHANNEL NUMBER

$10 \quad 16 \quad 17 \quad 18 \quad 19 \quad 20$

\section{0}

$334.0 \quad 334.0$

334.9334 .6

$\begin{array}{lllllll}335.5 & 335.5 & 335.3 & 336.0 & 336.1 & 336.0 & 335.3\end{array}$

$\begin{array}{llllllll}8.8 & 336.8 & 336.7 & 336.8 & 335.1 & 339.1 & 335.1 & 336.7\end{array}$

$\begin{array}{lllllllll}8.9 & 337.8 & 337.8 & 337.8 & 338.7 & 339.8 & 338.7 & 337.8\end{array}$

$\begin{array}{lllllllll}8.7 & 338.5 & 338.4 & 338.6 & 336.5 & 342.0 & 336.5 & 338.5\end{array}$

$8.9 \quad 339.3 \quad 339.3 \quad 339.5 \quad 340.0 \quad 342.3 \quad 340.0 \quad 339.5$

$84.3 \quad 419.0 \quad 419.8 \quad 422.0417 .6 \quad 412.4 \quad 417.5 \quad 422.2$

$104.8 \quad 451.0 \quad 451.7453 .9468 .6489 .2 \quad 468.5453 .06$

$\begin{array}{llllllll}118.6 & 188.7 & 489.7 & 488.3 & 468.0 & 461.9 & 468.2 & 487.8\end{array}$

$\begin{array}{llllllll}118.6 & 188.7 & 489.7 & 488.3 & 468.0 & 461.9 & 468.2 & 487.8 \\ 135.2 & 518.7 & 519.2 & 516.3 & 526.6 & 552.5 & 526.5 & 516.2\end{array}$

$\begin{array}{llllllll}135.2 & 518.7 & 519.2 & 516.3 & 526.6 & 552.5 & 526.5 & 516.2 \\ 138.4 & 551.3 & 551.3 & 545.2 & 515.1 & 508.3 & 515.2 & 545.3\end{array}$

$\begin{array}{llllllll}148.4 & 575.2 & 574.9 & 566.5 & 573.9 & 601.7 & 574.4 & 567.1\end{array}$

$140.8 \quad 598.9 \quad 598.2 \quad 586.6 \quad 551.4 \quad 544.6 \quad 554.0 \quad 588.4$

$\begin{array}{llllllll}144.7 & 615.3 & 613.5 & 600.3 & 604.8 & 631.9 & 606.6 & 602.7\end{array}$

$\begin{array}{llllllll}127.2 & 627.1 & 624.6 & 608.5 & 574.5 & 568.4 & 578.9 & 613.5\end{array}$

$\begin{array}{llllllll}123.3 & 632.9 & 629.1 & 612.5 & 614.2 & 637.4 & 617.7 & 617.6\end{array}$

$\begin{array}{llllllll}98.4 & 631.7 & 627.2 & 608.6 & 579.5 & 575.4 & 586.1 & 617.1\end{array}$

$\begin{array}{llllllll}87.6 & 626.8 & 621.0 & 603.0 & 601.9 & 618.9 & 607.8 & 611.2\end{array}$

$\begin{array}{llllllll}.0 & 549.2 & 542.8 & 524.3 & 510.5 & 513.3 & 519.5 & 535.3\end{array}$

$\begin{array}{llllllll}.0 & 544.3 & 536.8 & 519.1 & 511.8 & 514.1 & 520.5 & 530.2\end{array}$

.0. $540.6 \quad 532.4 \quad 517.6 \quad 513.8 \quad 517.0 \quad 523.3 \quad 530.4$

$\begin{array}{lllllllll}.0 & 539.1 & 531.0 & 518.3 & 516.0 & 520.7 & 527.2 & 532.6\end{array}$

. $0 \quad 537.8 \quad 529.6 \quad 518.9 \quad 517.4 \quad 522.4 \quad 528.9 .533 .6$

$.0537 .1529 .3 \quad 519.9 \quad 528.9 \quad 524.8 \quad 531.3 \quad 534.8$

$\begin{array}{llllllll}.0 & 536.3 & 528.5 & 521.2 & 521.1 & 527.6 & 533.8 & 536.7\end{array}$
HEAT FLUXES (KH/MZ)

OR SUB CHANNEL MUMBER

$10 \quad 16 \quad 17 \quad 18$

19

20 
CASE 1 CFIL BUNDLE - ONE SIXTH SECTION - FULLY ROUGH FLOH - 1/13/81

TIME $=$.OOOCO SECONDS TEMPERATURE DATA FOR ROD 8 IFUEL TYPE - CYLINDERI

FILM CLADOING TEMPERATURES IDEG-CI

$\begin{array}{cll}\text { OISTANCE } & \text { AVG FLUX } & \text { OROP-16 } \\ \text { (MM) } & \text { (KH/M2) } & \text { (OEG-C) }\end{array}$

10

11

For

$$
12
$$$$
13
$$$$
14
$$

15

16

$\begin{array}{llll}334.0 & 333.8 & 333.8 & 334.0\end{array}$

i 1991.2
186.0
430.0

430.0

554.8

714.6
805.8

805.8

991.2
1072.0

191.2

1272.0

1272.0

1391.2

472.0

1591.2

1672.0

1791.2

1872.0

37.847
37.847
37.847
37.847
37.847
37.847
655.718
782.385
936.682
1019.968
1105.298
1132.279
1136.591
1113.974
1034.548
955.458
804.880
681.017
.000
.000
.000
.000
.000
.000
.000

9.0

8.

8.

9.0
8.8
9.0

84.9

$105 \cdot 1$

119.0

135.3

138.6

148.5

140.8

144.6

123.1

98.2

87.4

87.4

2072.0

2179.1

2402.8

2544.2

2636.3

2742.3

2860.0
$337.7337 .531 .6339 .3-337.8$

$338.5338 .4 \quad 337.0 \quad 3.6 .1341 .8 .338 .6$

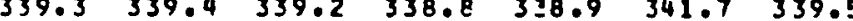

$\begin{array}{lllllll}419.8 & 421.9 & 423.0 & 418.0 & 420.3 & 406.1 & 422.8\end{array}$

$\begin{array}{lllllll}451.7 & 454.2 & 454.9 & 452.5 & 463.1 & 495.7 & 454.0\end{array}$

489.7493

519.2522 .1519 .451200518 .3561 .0851603

$\begin{array}{lllllll}551.8 & 554.7 & 548.8 & 533.8 & 516.6 & 502.2 & 545.1\end{array}$

$\begin{array}{lllllll}574.8 & 577.3 & 570.4 & 559.2 & 563.6 & 613.2 & 566.5\end{array}$

$\begin{array}{lllllll}59.8 .2 & 599.9 & 590.1 & 572.4 & 551.8 & 539.9 & 586.5\end{array}$

$\begin{array}{lllllll}613.5 & 615.2 & 604.6 & 591.0 & 593.5 & 644.2 & 600.3\end{array}$

$624.6 \quad 625.4 \quad 613.0 \quad 594.3 \quad 573.1 \quad 565.1608 .5$

$629.1 \quad 630.3 \quad 617.3 \quad 602.6 \quad 652.8 \quad 648.4 \quad 612.5$

$627.2 \quad 627.6 \quad 614.3 \quad 596.3 \quad 576.4 \quad 573.2 \quad 608.6$

542.0540 .0528 .0518 .5554 .9514 .0524 .3

536.0536 .052300513 .51504 .9514 .0

532.4532 .752509513 .1510 .7513 .8519 .1

532.4532 .7522 .9512 .8 51.8 .4514 .2517 .6

531.0 532.0 525.4514 .7 519.9 $515.3 \quad 518.3$

$529.6 \quad 531.7 \quad 525.5 \quad 515.0 \quad 511.0 \quad 515.7 \quad 518.9$

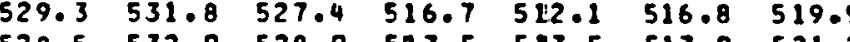

$621.0 \quad 622.1608 .4594 .159106026 .760300$
DATE 27 MAY 81 TIME $11: 21: 28$

HEAT FLUXES $(K W / M Z)$

FOR SUBCHANNEL NUMBER

$14 \quad 15$

16 
DUCT TEMPERATURE RESULTS IOEG-C)

CASE 1 CFTL BUNDLE - ONE SIXTH SECTION - FULLY ROUGH FLOH - 1/13/81

TIME $=.00000$ SECONDS

DISTANCE CHANNEL CHANNEL DISTANCE CHANNEL
(MMI.

991.2

1072.0

1191.2

1272.0

1391.

1472.0

1591.2

1672.0

1791.2

1991.2

2072.0

\subsection{9}

324.6

355.1

347.5

385.1

385

378.6

419.5

415.5

454.1

452.7

485.3

CHANNEL
151

CHANNEL (17)

CHANNEL 1191 1191

CHANNEL $(21)$

CHANNEL $(22)$

CHANNEL 1

$\begin{array}{llllll}320.6 & 324.7 & 3.19 .8 & 325.0 & 320.6 & 326.6 \\ 345.2 & 330.4 & 335.8 & 330.5 & 345.1 & 331.6\end{array}$

$\begin{array}{llllll}302.8 & 319.1 & 300.5 & 319.5 & 302.6 & 325.8\end{array}$

361.9

$\begin{array}{llll}322.7 & 341.5 & 319.0 & 342.9\end{array}$

372.0340 .0237509

$354.0 \quad 372.8 \quad 349.0 \quad 375.9 \quad 354.4 \quad 386.9$

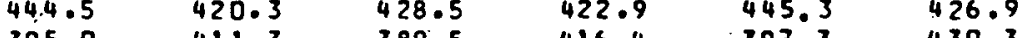

$\begin{array}{llllll}395.0 & 411.3 & 389.5 & 416.4 & 397.3 & 430.3\end{array}$

$\begin{array}{llllll}478.1 & 456.3 & 463.4 & 460.7 & 481.2 & 468.1\end{array}$

$\begin{array}{llllll}438.3 & 450.9 & 433.5 & 458.2 & 444.0 & 474.8\end{array}$

$\begin{array}{llllll}505.7 & 488.9 & 494.7 & 495.6 & 512.6 & 506.8\end{array}$

ITERATIONS $=13$

$\stackrel{?}{\mathfrak{u}}$

SPEC:FIEO NUMBER OF TIME STEPS COMPLETEO

TOTAL ELAPSED. TIME IS 2 OL SECONDS.

* \#EXITLOC $\$ 034727$

aFIN 


\section{THIS PAGE WAS INTENTIONALLY LEFT BLANK}


APPENDIX D

LISTING OF COBRA/CFTL CODE*

The encloscd listing is for the COBRA/C.FTI. conde after it was made operational on the GA's UNIVAC computer and contains calls to the following GA system subroutines.

$$
\begin{aligned}
& \operatorname{DATGT2}(A): \text { current date } \\
& \operatorname{GTIME}(T): \text { current time } \\
& \operatorname{TICKER}(X): \text { elapsed SUP time }
\end{aligned}
$$

When using the COBRA/CFTL code on other computing systems, call to above routines must be replaced by the call to equivalent routines.

$$
\text { D-1 }
$$




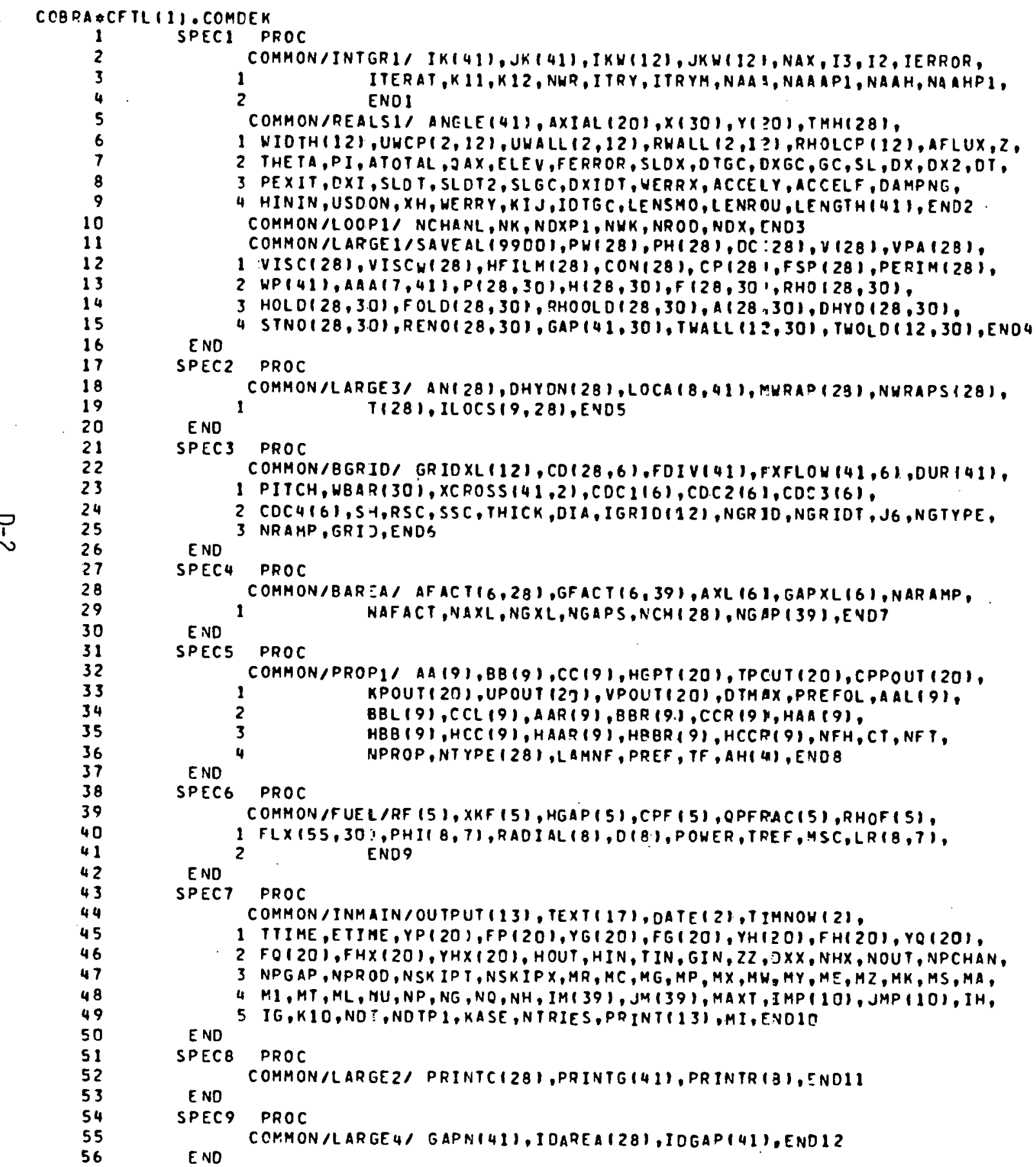


SPECIO FROC

COMMON/MIXI/ ABETA, BBETA, GK,FTM, J5,NSCBC, ENDI 3

SPECII PROC

COMMON/EOFIX, GAPS $(28,4)$, ENOL14

END

SPEC12 PROC

END

COMMON/RADVI NSF, IRAD, ICON, NCONR, IFL, IJM, ENDIS

SPECI3 PROC

COMMON/GRP13A, Q2(9),RZS(3),RR(9), HRIB, THTB,BIOIC,BIOT,ENDI6

ENO

SPF.C14 PROC

END

COMMON /GRP 138/ LREFF, LREF ST, AR, AHS, GPRIME, HPLUS, ENDI7

SPEC15 PROC

ENO

COMMON/GRP I3C/ CRIBI, CGIBI, END18

SPEC16 PROC

ENO

COHMON/ICONR, ICONR(8), ENOI9

SPEC 17 PROC

ENO

COMMON/PR/ PR,END2O

COMMON/QPRI/ OPRIM (28),END21

SPEC2O FROC

COHMON/SIZES/. AC (28), OR (B), HINLET(2B), TINLET(28),

ENO

1. FINLET 28, ,OIST128,4:,LC128,4), ENDZ2

SPEC21 PROC

COMMON/RAD/OR (55,30), TSF (55), HFA(550), I IS (550), JJS(55O), END23

ENO

COMMON/HFLX/ FLUX 18,301, END 28

END

SPECZ3 PROC

COMMON/LAMNAR/LAMARK 1281 ; TETA 281 , ENO 24

ENO

SPEC24 PROC

END

COMMON/RUF F /HP $(28,30)$, RTPLS 128,30$)$, END 25

SPEC25 PROC

COMMON/ERRORI/ISAVT, ISOUT,OIM,OOM,ENO26.

SPEC26 PROC

ENO

COMMON/ICONT/ICOUNT, ENOZ:

SPECSI PROC

DIMENSION UB $1330,301,5$ TUU 1330,301, AP 1330,301

SPEC52 PROC

DIMENSION WI 330,301, HOLO: 330,301, BETA 1330,301, BSAVE $(330,30)$,

ENO

PSI 1330,301, IRF 1330,301

SPEC5 3 PROC 
DIMENSION US 1330,301, USH 1330,301, TERM $1(281$, IERM2 (28), OPK 1281, APPI330,301, FLON1281, E(41:, C1J141):

2 MARK $(330,30)$, SP $(330,30)$,OPWP 281 , IFOIVI4)

END ISTAZ (41), AAHI287), ILOCI252), WPPI41), DSHIFI (28)

SPEC55 PROC

END

DIMENSION HFILMS 1281

SPEC56. PROC

ENO

DIMENSION DPOX $(28)$, OHOX(28), DFOX(28), CONK 1411$)$

SPEC5T PROC

DIMENSION AAAPRM $(287)$, UPRM199001, LOCA1(328)

DIMENSION USHPRM 1411 ,USPRM(41), BETPRM (41), SPPRH 1411

1

END

WOLOPM $(41)$, UBPRM(28), APPPRH $(28)$, WSAVE $(41)$,

SPECSE PROC

NO

END
SPECSO PROC

$E N D$

OIMENSION TFL $\times(55,30)$

SPECTI PROC

1

EOUIVALENCE 1 U8 11,11 , SAVEAL $(247) 1$, 15 TUH 11,1$)$, SAVEAL $(2751)$, IAPI1,11, SAVEAL 13031$)$

SPECT2 PROC

EOUIVALENCE (W11,1), SAVEALI1), (HOLOI1,1), SAPEAL (42)1,

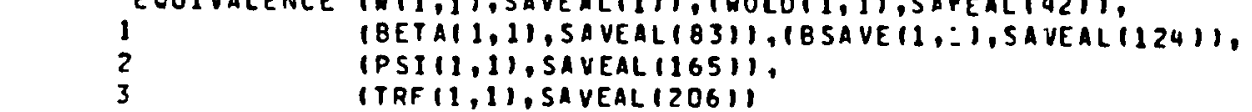

END M, Saveal $11651 \%$

SPECT3 PROC

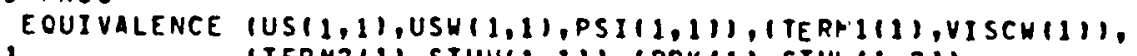

1 ITERM2 II,STUHI1,111, IOPK I1),STUL $11,2 \%$,

(APP 11,1$), A P(1,1),(F L O W(1), 5$ TUH 1 1,3),

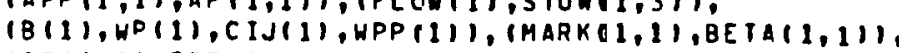

ISPI1,1), TRF 1 1,1)1, IAAHI11, AAAI 1,11),

IILOCI1), ILOCS(1,1), ICPWPII),FSP (1)

6 IPSHIFIIII,TMHIIII, IISTARIII, JFOIVIII,FOIVIII

END

SPEC76 PROC

EOUIVALENCE IOHOXI1),CP1111, (OFOXI1),FSP(11), $1000 \times 111, C O N 1111$,

END ICONKIII,AAAI I, III

SPECT7 PROC

EOUIVALENCE IAAA 11,1$),$ AAAPRM(1), IN(1,1), WPRM(1)1,

1

ILOCAI1,1),LOCAI111:

EOUIVALENCE I WSAVEI 11 , BSAVE $11,311,1011111)$, BSAVE 11,111,

1 IOTJJI1,BSAVEI1,211, (USWPRMI1),USWI1,111,

(USPRM $(1)$,US $(1,1)$, (BE TPRM 11$), B E T A(1,1)$ ),

(SPPRM I 1),SP(1,1)1, IWOLDPM(1), WOLDI1,11),

END

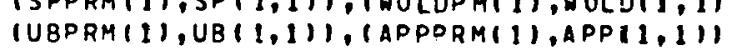

SPEC6O PROC

$M C=28$ 


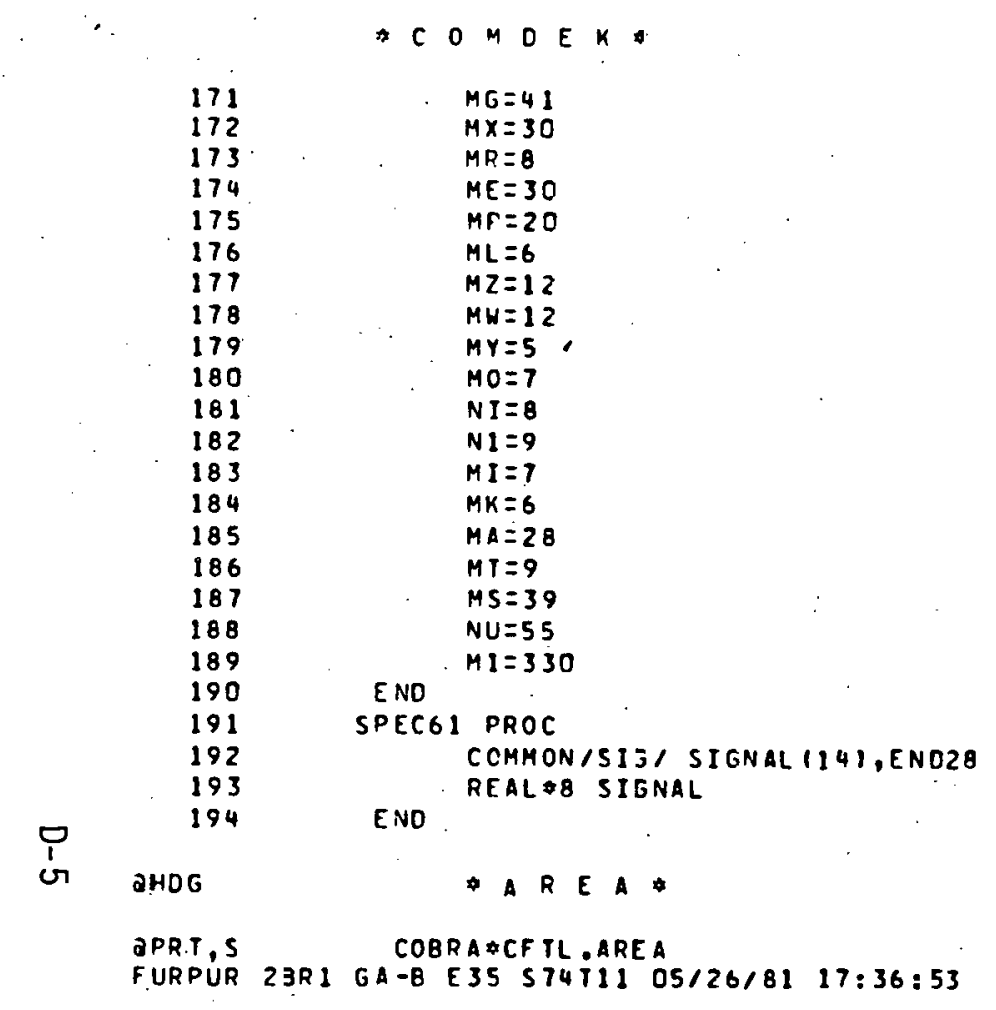

COBRA $\$ C F T L$ TARE 
COBRA $*$ CFTLI1).AREA

SUBROUTINE AREA $\mid J, J X$ )

INCLUDE SPECI,LIST

INCLUDE SPEC2,LIST

INCLUDE SPEC3,LIST

INCLUDE SPEC4,LIST

INCLUDE SPECQ,LIST

INCLUDE SPECSI,LIS:

INCL UDE SPECS2,LIST

INCL UDE SPECS3,L IST

INCLUDE SPECT1,LISI

INCLUDE SPEC72,L ISI

INCLUDE SPEC73,L IS

LOGICAL GRIO

REAL IOTGC,LENGTH,KIJ,LENSMO,LENROU

ICROSS $(K)=I F I X$ IEINTEG *PI TCH $* 1 B S(X C R O S S(K, L))$

c

$I X C(K)=1(1 \times J X-I C R O S S(K)) / I P I T C H) * I P I T C H+I C R O S S(K)$

$F F S=F F$

$J H 1=J-1$

JXMI $=J X-1$

If (J.NE.1) GO TO 100

$P L=0$

JXM1 $=1$

JMI $=1$

\section{CONTINUE}

EINTEG REDUCED TO $1.0 E 9$ TO AVOID FIXED POINT OVERFLON

EINTEG $=1.0 E 9$

IXJX IFIXIEINTEG $X X(J X)$

I $X J X M I=I F I X(E I N T[G * X(J X M I))$

IPITCH I IF IXIEINTEG $\triangle P$ ITCH

CALCULATE CHANNEL AREA IF REQUIRED.

DO $110 \quad I=1$, NCHANL

$A(I, J)=A N(I)$

OHYOII,JI=DHYONIII)

110 CONTINUE

If INAXL.EO.OI GO TO 130

DO $120 I=1$, NCHANL

JJ=IDAREAIIS

IF (JJ.LT.J) GO TO 120

CALL CURVE (FF, XIJXIIZ, AFACTII,JJI,AXL, NAXL,IERFOR, 1 )

IF IIERROR.GT.11 GO TO 240

DUMY = FLOATIITERATI /FLOATINARAMP)

DUMY = AMINI (OUMY, 1.)

IF IFF.LE.O.I GO TO 240

$F F=1 .-|1,-F F|+D U M Y$

$A(I, J)=A N(I)$ \&F

C FF DECREASED IN SMOOTH SECTION TO ACCOUNT FOR PH EFFECT ON DHYO IFIFF.LT,0.99IFF =FF/1.0171

IFIFF, LT, O.99IFF $=F F / I$
OHYDII, JIIOHYDNIII $\$ F F$

120 CONTINUE

130 IFIJ6.NE.1.AND.J6.NE.3) GO TO 210

IF INK.EO.O) GO TO 231

IF IJX.NE.11 60 TO 150

DO $140 \quad I=1$, NCHANL

140 NWRAPII I =NWRAPSII)

150 CONTINUE

203500

203600

203700

203800

204100

204000

204100

204200

204300

204400

204500

204600

204700

204800

204900

205000

205100

205200

205300

205400

205500

205500

205700

205800

205900

206000

206100

06200

206300

206400

206500

206600

206700

206800

206900

207000

207100 
DUMY SFLOATIITERATI/FLOATINARAMPI

207200

207300

WPUU =PIOTHICKODUMY

207400

A WW $=$ WP WWATHICK 0.25

207500

C PL IS THE PITCH LENGTH CONTAINING XIJ1. 207600

FIND THE HRAP CROSSINGS IN DX.

IF IJX,EQ, I,OR .JX,EO,NDXP 11 GO TO 190

$\begin{array}{ll}00180 K=1, N K & 207800\end{array}$

$\begin{array}{ll}I I=I K(K) & 208000\end{array}$

$\begin{array}{ll}J J=J K(K) & 208100\end{array}$

$\begin{array}{ll}\text { DO } 180 L=1,2 & 208200\end{array}$

If IXCROSSIK,LI.EO.R., GO TO $180 \quad 208300$ C AOD AND SUBSTRACT HIRE WFAPS FROM SUBCHANNEL 190 T EACH WRAP CROSSING 208400 IF IXCROSS IK,L II $160,180,170$

160 NURAPIIIIIENRAP(II)+1 208700

NHRAPIJJI=NHRAPIJJI-1 $\quad 208700$

$\begin{array}{ll}\text { GO TO } 180 & 208900\end{array}$

$\begin{array}{ll}170 \text { NWRAPIII }=\text { NHRAPIII }-1 & 209000\end{array}$

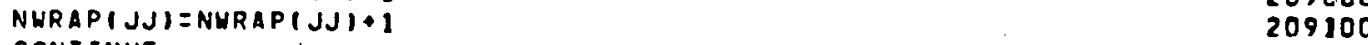

$\begin{array}{ll}180 \text { CONTINUE } & 209200\end{array}$

190 CONTINUE 209300

C MOOIFY AREA ANO HYOPAULIC DIAMETER FOR VIRE WRADS IN SUBCHANNELS 209400 DO $200 \quad I=1$ NCHANL

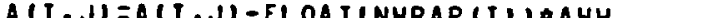
$\begin{array}{lll}200 \text { DHYD }(I, J)=4 . * A ! I, J) /(P E R I M(I)+F L O A T(N H R A P(I) \text { IIPWH } & 209700 \\ & & 209800\end{array}$

TCULATE GAP SPACING IF REQUTREO. TH.EO.OT GO TO 23 DO $220 K=1, N K$

220 GAP $(K, J)=G A P N(K)$

IFINGXL.EO.OI GJ TO 231

DO $230 K=1$, NK

$L=I D G A P(K)$

IF (L.LY.1) 60 TO 230

CALL CURVE (FF, XIJX)/2,GFACT (1,LI,GAPXL, NGXL, IERROR, 1)

IF IIERROR.GT. 11 GO 10240

IF IFF.LE.0.1 GO TO 240

GAP $(K, J)=G A P N(K)$ \&F

230 CONTINUE

231 IFIJ.EO.11GO 10 233

DO $232 \mathrm{Y}=1, \mathrm{NCHANL}$

232 APPII,JI=.5*IAII,J)・AII,JMI)

210000

210100

210200

210200

210300

210400

210500

210600

210700

210800

210900

211000

211100

233 CONTINUE

FF $=$ FFS

RE TURN

240. IERROR $=9$

$F F=F F S$

RETURN

$\begin{array}{rrr}105 & \text { RETURN } & 211800 \\ 106 & \text { ENO } & 211900\end{array}$

211300

211400

211.500

211600

211700

$\begin{array}{llr}105 & \text { RETURN } & 211800 \\ 106 & \text { ENO } & 211900\end{array}$ 
COBRAFCFTLI1).BASIC

SUBROUTINE BASIC IRO,RI,HR,RE,TR,F,ST,I,JI C SUBROUTINE TO CALCULATE FRICTION FACTOR ANO STANTON NUMBER FROM R

CH = RELAXATION PARAHETER

DH - HYORAULIC DIAMETER

F - FRICTION FACTOR

FI = STARTING VALUE OF THE FRICTION FACTOR

GH - FUNCTION IN THE TEMPERATURE PROFILE

HR - RIB HEIGHT

REW - REYNOLOS NUMBER AT WALL

RH - FUNCTION IN THE VELOCITY PROFILE

RO - RADIUS OF ZERO SHEAR

R1 - RAOIUS OF ROD

ST. - STANTON NUMBER

$Y H-R O-R 1$

INCLUDE SPEC 14, LISI

INCLUDE SPECI5,LIST

INCLUDE SPECIT,LIST

INCLUDE SPEC24, LIS T

$\mathrm{CH}=.7$

IFITR .LT. 1.1 TR=1.

$R E W=R E T R * 1-1.671$

$Y H=R O-R I$

$O H=2 .: 1 R O \& R O-R I * R 1 \mid / R 1$

$F I=.1$

009 I $T=1,20$

$H W=H R / O H \$ R E H \$ S O R T I F I / 8.1$

$R H=C R I 11$

$A R=C R(2)$

IFIHH.GT.CRI3)1GO TO 11

$R H=C R(4)=C R(5) * A L O G(H H)$

$A R=C R(6)-A L O G(H W)$ (CR $(7)-C R(B)+A L O G(H W))$

$H H=H H 1 R+1.67$

RHSM $=5.5+2.5 * 4$ LOG (HH)

95900

96000

96100

96200

96300

96400

96500

96600

96800

96900

97000

97100

97200

97300

97400

97500

TEST $=R H+(A R-2.50) \neq(A L O G(Y H / H R)-.5-1.1(2 .+Y H / F 1) 1$

IFITEST, RHSM FLOW IS HYDRAULICALLY SHOOTH

IF (TEST.LT.RHSMI GO TO 11

RHERHSM

$R=2.50$

$11 C_{4}=A R$ Q $O L O G|Y H / H R|$

$C 5=A R+11.50 * 0.50 \% R O / R 11 / 1100 R 0 / R 10$

$C 61=1.1\left(C_{4}-C_{5}+R H\right)$

$F=1$ C6I*2148.

$F=\mid 1,-C H \| F I+C H \# F$

IFIABS $(F-F))$. LT. .COI) GO TO 10

$F I=F$

CONTINUE

PPINT 100

100 FORMATI'O BASIC NOT CONVERGED.

RETURN

97800

97900

98000

98200

98300

98500

98600

98900

99300

10 CONTINUE

HPW $(I, J)=H W$

99700

100000

100100

$\ln 0200$

100300

100300

100400

100500

100600

100700

100800 
- B A 5 I C*

DATE 052681

PAGE

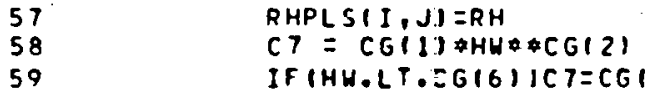

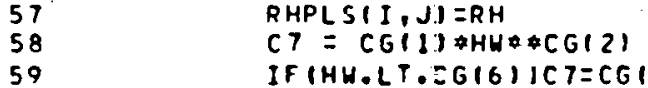

59 IFIHH.LTEEGI6)ICT=CGITI

$60 \quad C 8=P R A Q G(4)$

$C Q=T R+C G 15$

$G H=C 7 * C 9 * C 9$

$A H S=C G(3)$

$S T=C G I /(A H S / A R *(C 4-C S)+C H)$

RETURN

ENO

101200

65

केHDG

- BEF

COBRA \#CFIL.BEF

FURPUR 29R1 GA-B E35 574T11 05/26/81 17:36:53 
COBRA $\#$ CFTL 11$). B E F$

SUBROUTINE BEF (AL, RE, F,AS,AR)

C INNER AND OUTER ZONE DENSITIES ANO VISCOSITIES ISSUMED EOUAL

$R 1=1$.

$R 2=1$.

ET1 $=1$.

$E T 2=1$.

RE TURN

ENTRY BEF $1(B, Y)$

$W F=S Q R T(2.0 / F)$

$B 2=1.0-B \star B$

$A 1=1.0-A L$

$A 2=1.0-A L * A L$

$B A=B+B-A L+A L$.

$B A A=B A / A L / A 1$

$G A=B / A L$

WEA $=$ SORT (B2*AL/BA)

$32=A S / 2.0 *(3.0+B / 1 / 1.0+B)$

$G 1=A R+(1.50+0.50 * 6 A) /(1.0+G A)$

$A A=A S * A L O G(11.0-B) / 2.0 / A 1$ *SORT(B2/A1)*RE/HF \#ET2/R2)+5.5

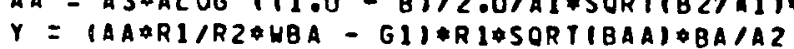

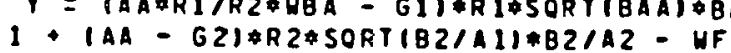

RE TURN

ENTRY BEF4 IF I,F 2, RE 2, RE 2 I

$F 1=2.0 /(A A+W B A \$ R 1 / R 2-61) * 2$

$F 2=2.0 / 1 A A-G 2) \# 2$

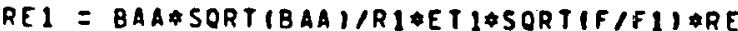

REZ = BZ/AI*SORTIBZ/AI)/R2*ET2*SORTIF/F $Z$ I*RE RETURN

300

END

500

600

700

800
900

1000

1100

1200

- B E T A F

1700

1900

2000

2100

2200
2300

2300

2400

2500

2600

aHDG

APRT, 5
FURPUR 28RE GABRA

FURPUR 28R1 GA-B E 35 $574 T 11$ 05/26/81 17:36:54 
- BE TAF*

COBRA OCF TLIII.BETAF

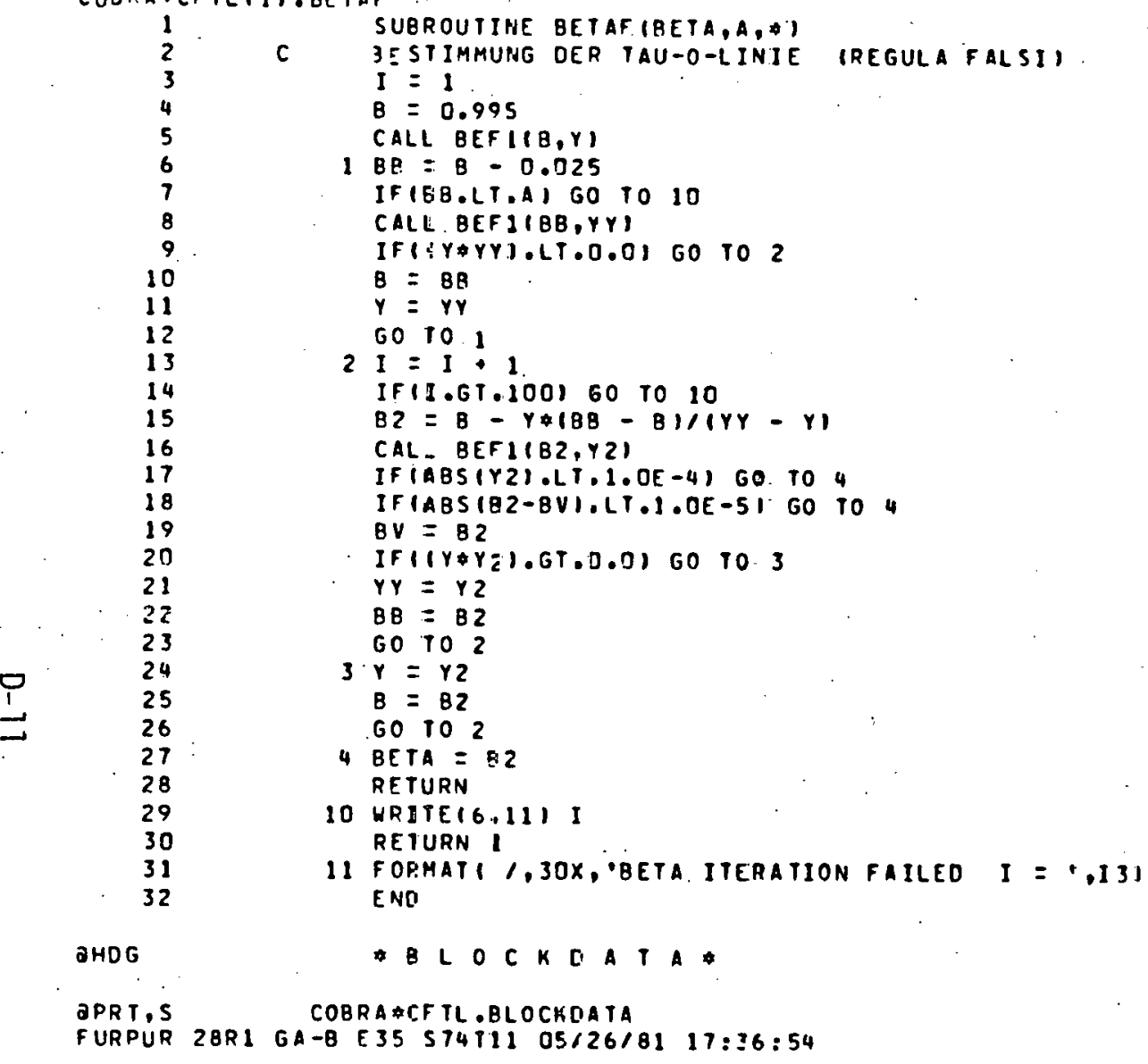


COBRA OCFIL11).BLOCKOATA

BLOCK DATA

INCLUDE SPEC6I,LIST

DATA SIGNALI COBRA

1 - SCHEME $\because$ FORCE

2

$\because$ DIFFER " ' OIVERT $\because "$ MIX $\because$ YOID END

331000

QHDG

- C L D T H P *

DPRT, $5 \quad$ COBRA \#CF TL, CLDTMP

FURPUR 28R1 GA-B E 35 S74T11 05/26/81 17:36:54 
COBDA*CFTL(1), CLOTMP

SUBROUTINE CLOTMPIJX,NI

INCLUDE SPECL,LIST

INCLUDE SPEC2, LIST

INCLUDE SPECS,LIST

INCLUDE SPECG,LIST

INCLUDE SPECT,LIST

INCLUDE SPEC12,LIST

INCLUDE SPEC59,LIST

LOGICAL PQINT

REAL KPOUT

REAL IOTGC,LENGTH,KIJ,LENSHO,LENRO

THIS ROUTINE CALCULATES THE CLADDING SURFACE TEMPERATURES.

c DIMENSION ISUB 171 , TCLDIT

IFIJX.GT. NSKIPXI 60 TO 20

FIND SURFACE WUMBER NS

$N S=0$

$N 1=N-1$

IFINI.LT.1) 60 TO 16

DO $251=1, N 1$

DO 15 II $=1, M S C$

IFILRII,III.6T.0I NS $=N S+1$

15 CONTINUE

16 CONTINUE

LO $=M S C$

DO $10 L=1$, $M S C$

$I S U B(L)=L R(V, L)$

IFIISUB(L).GI.0I GO TO 10

$\angle O=L D-1$

10 CONTINUE

WRITE $(6,959)$ ISUB (LD), IISUBIL),L $=1, L D$

WRITE $(6,101) 1$ ISUB(L),L $=(, L D)$

WRITE 16,2821

O CONTINUE

$x J=x(j x) * 1000$.

IFIIFL.EO.0.OR.JX,E0.1);0 TO 48

$O X=x(J x)-x(7 x-1)$

CALL CURVE 1OX1, $X 1 J X-11 / 2, A X I A L, Y, N A X$, IERROR, 1

CALL CURVE $(O X 2, X(J X) / Z, A X I A L, Y, N A X, I E R R O R, 1)$

TEST $=(0 \times 2+1 . E-5)(10 \times 1+1, E-5)$

IFITEST.LT.3..AND.IEST.3T.0.33IGO TO 17

$0 \times s u m=10 \times 1+0 \times 2) 12$

$D \times F=0 \times 120$

$X F=X(J X-1)$

$001110 X F=1,19$

$X F=X F \bullet D X F$

CALL CURVE IOX,XF /Z,AXIAL, Y,NAX, JERROR, 11

11 OXSUM $=O X S U M+Q X$

$0 \times=0 \times \operatorname{SUM} 120$

60 TO 48

17 XCUMY $=(x)(J X)-0 x / 2,1 / 2$

CALL CURVE (OX, XDUYY, AXIAL,Y,NAX, IERROR, 1$)$

48 CALL CURVE IOAX,XIJXI/Z, AXIAL,Y,NAX, IERFOR, 1 I

DFLUX = AFLUX OAX\#RADILLINI \#POJER

260400 
PRMTOT
PRT

$0050 \mathrm{~L}=1, \mathrm{LO}$

$I=$ ISUPIL)

CALL CURVE ITHEL,HE I , TIPOUT

CALL CURVETTHEL,HII,JI,TPOUT,HGPT, NPROP,IER'ROR,11 262200

CALL PROPI $2, J, J X, I, I)$

IFIIFL.EO.0IGO 1049

262300

IF IJX.GT.1.AND.OX.GT.O.OO 1/QRAD =QAX $10 X$

IFIORAD.EQ.0.1GO YO 46

TFLX(NS $+L, J X)=F L X(N S+L, J X) \neq O R A D$

OFLUX $=I F L X(N S \bullet L, J X)$

60 TO 47

46 CALL PROPI2,J,JX, I, NCHANL

CALL ROOCONINROD,JX, T, HFILM,O.,DT,

IFIIRAD.GT,OICALL RAOIATIJXI

$T F L X(N S+L, J X)=F L X(N S+L, J X)$

OFLUX $=T F L X(N S+L, J X)$

$47 F L X Y O T=F L X T O T+D F L U X \# P H I I N, L)$

PRMTOT =PRMTOT+PHIIN,LI

49 OTFLM = OFLUX

TCLOILI = THEL DTFLM

50 CONTINUE

IFIIFL .EO \& IIOFLUX $=F L X T O T$ I PRM TOT

262600

PRINT HEAT FLUXES FOR EACH SUPFACE OF THE ROJ

IFIIFL.EO. OIRE TURN

$00466 \mathrm{~L}=1, \mathrm{LO}$

466 OUTPUTILI = TFLXINS+L, JXI

WRITE $(6,963)$ IOUTPUTIL),L=1,LD

RETURN

101 FORMATIIH+,79X,7I7!

280 FORMAT IF $7,1, F 13.3, F 9,1,1 \times, 7 F 7.1$ )

282 FORMAT IIH

959 FORMAT $/, 41 X$, CLADDING TEMPERATURES IDEG-CI $, 1,24 X, 4 \mathrm{HFILH,}$ 68X, 32HHEAT FLUXES IKH/HZ

28H OISTANCE

$.29 \times, 21$ HFOR SUBCHANNEL NUMBER,

.3OH (MM) (KH/H2) (DEG-C),15,6I)

963 FORMAT $11 \mathrm{H}, 79 \times, 7 F 7,21$

ENO

264700

265000

265100

265200

265200

265800

265900

266100

266400

266500

266600

266700

266900

จHDG

DPR T, S

- co B R a.

COBRA $\#$ CF TL. COBRA

FURPUR 28R1 GA-B E35 S74T11 05/26/81 17:36:55 


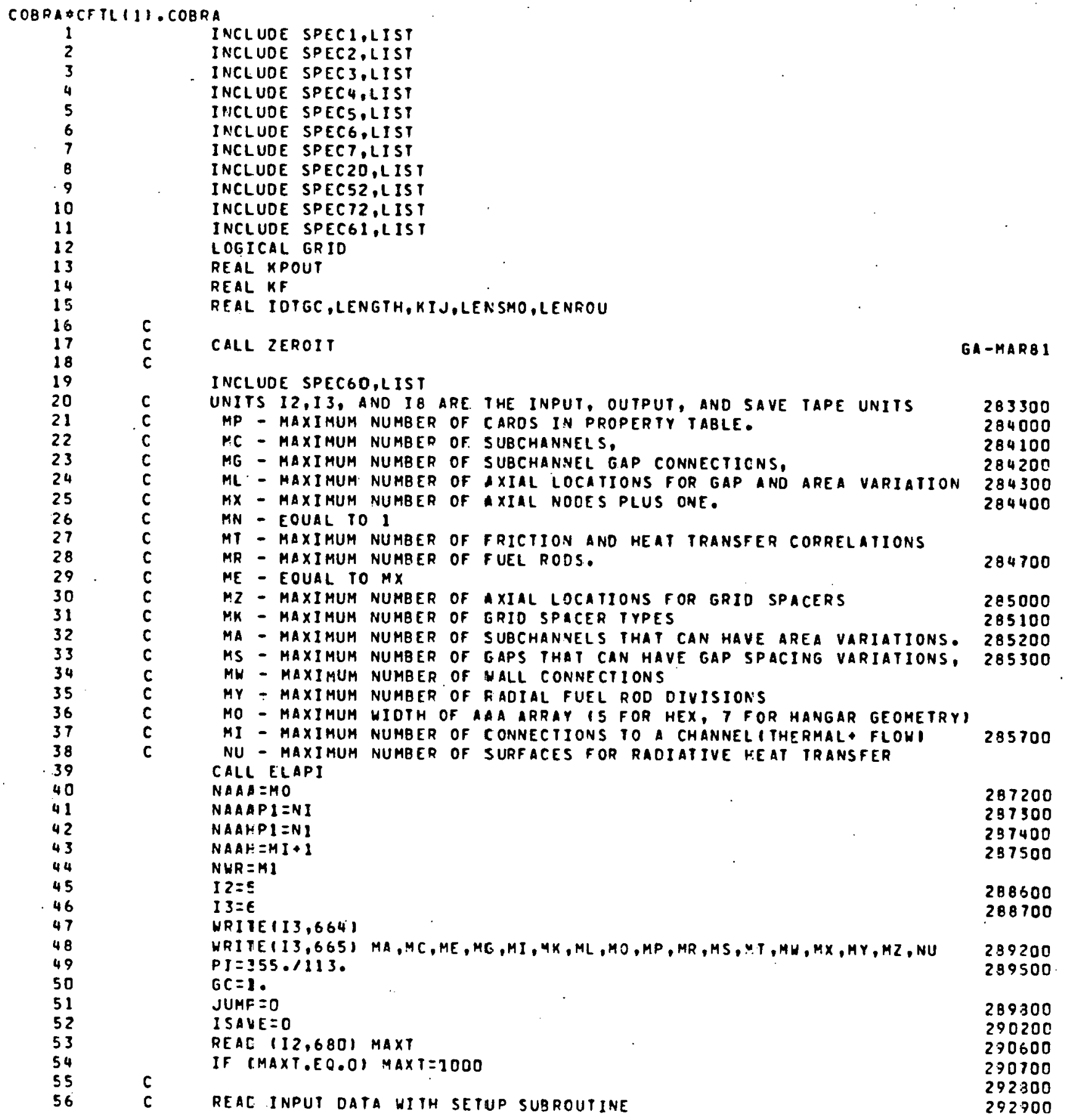


110 call setup

IF IIERROR.GT, O.OR,KASE.LT.LI STOP

SAVEOT $=D T$

NOTP $1=$ NOT +1

START TIME LOOP

c

\section{DO $650 \mathrm{NT}=1, N D T P 1$}

IERR OR $=0$

IF INT EEO.1) OT $=1 \cdot E+10$

DO I40 $I=1$,NCHANL

ANIIIIICII)

DHYONIII $=D C$ II

140 PERIMIII $=P W I I)$

IF INT.NE.2I GO TO 150

NRAMP $=1$

NARAMP $=1$
DT=AMINI IOT, SAVEDTI

150 CONTINUE

IF INT.EO:1) ETIME =0

293000

293100

293300

293500

293800

293900

294000

294600

295300

295500

ESTABLISH CHANNEL BOUNDARY CONOITIONS AND FORCIVG FUNCTION VALUES.

IF (NP.GT.1) CALL CURVE IDUMY,ETIME,FP, YP,NP, IEPROR, I)

IF (IERROR.GT 1 I) $G O$ TO 660

PREF = DUMY PEXII

DUM =ABSIPREF-PREFOLI/PREF

IF IOUM.LT,.OOOII GO TO 160 PREFOL =PREF

$T F=T I N-5$.

CALL PROP $(1,1,1,1$, NCHANL)

160 CONTINUE

IF IIERROR.GT.1I GO TO 660

DUMY $=1$.

IF INH.GT. I.) CALL CURYE IOUMY,ETIME, FH, YH, NH, IERROR, I)

IF IIEFROR.GT.II GO TO 660

DO $170 \quad I=1$, NCHANL

HOLO (I, 1) $=H(1,1)$

HII, II IHINLETIII $\$$ DUMY

H(I, II =HINLETIII \$DUMY
IF IIH.NE. I.AND.IH.NE.3) GO TO 170

IF IN $=$ IINLET(I) $\$$ DUMY

VIN=IINLET(I) $\$$ DUMY

$H\{I, 1\}=$ VOUT

$170 \mathrm{HI}=H(1,1)$

CALL CURVE IVOUT, HI, TPOUT, HGPT, NPROP, IERROR, 1 I

CALL CURVE IVI,HI, VPOUT, HGPT, NPROP, IERROR, 2 I

$T(I)=V O U T$

RHO $(I, 1)=1, / V I$

190 CONTINUE

220 DUMY $=1$

IF ING.GT. II CALL CURVE IOUMY, ETIME,FG,YG,NE, IERROR, I)

IF (IFRROR.GT.1) GO TO 660

DO $300 \quad I=1, N C H A N L$

$300 F(I, 1)=F I N L E T I I) \neq D U M Y$

DUMY $=1$.
296200

296300

296500

296600

296700

296800

296900

297000

297100

297200

297800

297900

298000

298200

298300

298400

298500

298600

298700

298800

298900

299000

299100

299200

299500

299600

300400

300400

300500

300600

300700

301100

302200

302300

302400

304000

304100

304100 
If INO.GT.1I CALL CURVE IDUMY,ETIME,FO,YO,NO,IERROR, 1) 304700 IF IIERROR.GT.1I GO TO 660 POETIME,FO,YONN,IERROR, II 304800 $\begin{array}{ll}\text { POWER }=O U M Y & 304900\end{array}$ IOTGC=1.1(OT\#GC) $\quad 308500$ DTGC =DTOGC $\quad 308600$ SLDT $=S L / D T$ SLOTZ=SLOT/OT

C IMPLICIT SOLUTION IF IERROR OGT IRIES, JUMP

CRROR.GT.II GO TO 530

IF IJUMP OLT I OR JUMP GT - II GO TO 660 IF (JUAP GE, 2) GO TO 530 URITE 113,6701 ITR IES IERROR $=1$

$53000620 \mathrm{~J}=1$, NDXP1

IF (J.EO.1) FININEFI1,J)

IF (J.EO.NDXPI) FINOUT $=F(1,3)$

IF INK .EO.OI 60 TO 570

$00560 K=1, N K$

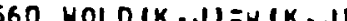

570 CONTINUE

IF (J.EQ.1) HININEHI1,1

DO $580 \quad I=1$, NCHANL

FOLD $(I, J)=F(I, J)$

$H O L D(I, J)=H(I, J)$

RHOOLD (I,J) $=R H O(I, J)$

580 CONTINUE.

If (NHK.EQ.0) 6010600

DO $590 \quad K=1$. NWK

590 THOLD(K,J) $=$ THALL $(K, J)$

600, CONTINUE

620 CONTINUE

I SAVE = IERR OR

$c$

311000

311100

311300

319300

319400

319500

319600

319800

320000

320100

320300

320400

320500

320600

320700

321300

322000

322000

322100

322200

PRINT RESULTS WITH SUBROUTINE RESULYINT; $\quad \begin{array}{r}323300 \\ 323400\end{array}$

CALL RESULTINTI

C

IF IIERROR.GT.O.OR.ISAVE.GT.01 GO TO 660

323600

323700

650 CONTINUE

323800

WRITE $\{13,720\}$ IF (IERROR.GT.O) WRITE II3,690I SIGNALIIERRORI
IF IISAVE.GT.O) HRITE (I3,690I SIGNALIISAVE) CALL ELAPIMTINE)

HRITEII3,740) MIIME

c

GO TO 110

326700

326800

664 FORMATI1H1,15X,201' $1,11,34 X, 0$ THIS IS THE OAK RIDGE NATIONAL ILABORATORY VEFSION OF, THE, /, $38 \times, 49 H C O 3 R A-I V$ THERMALI - HYORAULIC ANALYSIS COMPLTER COOE, $/ /, 24 \times, 62$ HADAPTED FROM THE BNHL - PPOGRAM COBRA-IV-I WITH UPOATES THROUGH, "FX 15\%,7H DATED. 
665 FORMATI27HOCOBRA DIMENSION PARAMETERS

-5H MA $=15,5 X$ 'SUBCHANNELS HITH AREA VARIATIONS GRP $5 \% 327800$

\5H MC $=15,5 \times$ ' SUBCHANNELS

GRP $4 \% 327900$

* $5 H$ HE $=[5,5 \times \cdot 1=M X]$

-5H MG $=15,5 \times$. SUBCHANNEL gaP cONNECTIONS

GRP $9 \% 1328000$

*5H MI $=I 5,5 X$ ICONNECTIONS TO CHANMEL ITHERMAL +FLOHI

GRP $4^{\circ} / 328100$

+5H MK $=15,5 X$. GRID SPACER TYPES

GRP 0.1328200

\$H MK $=15,5 X^{\circ}$ GRID SPACER TYPES

\#5H ML $=I 5,5 X$, AXIAL LOCATIONS FOR GAP AREA VARIATIONS

GRP $7 \% 328300$

-5H MO $=15,5 X$. WIOTH OF AAA ARRAYITRIANGLE=5, HANGAR=7)

GRP $5 \% 328400$

\% 5H MP $=15,5 X \cdot$ CARDS IN COOLANT PROPERTY TABLE

GRP $0 \% 328600$

-5H MR $=15,5 \times \cdot R O D S$

-5H MS $=15,5 X$ 'G APS HITH GAP SPACING VARIATIONS

GRP $1 \% 1328700$

-5H MT $=15,5 X$. CORRELAITIONS

GRP $8 \% 328800$

GRP $6 \% 328900$

- 5H MH $=15,5 X$. THERMAL CONNECTIONS

GRP $6 \%$ GR $2 \% 1328900$

5H MH $=15,5 X$. THERMAL CONNECTIONS

*5H MX $=15,5 X \cdot A X I A L$ NODES PLUS ONE
SH MY $=15,5 X \cdot R A D I A L$ ROD DIVISIONS

\# 5H MZ $=15,5 X$.AXIAL LOCATIONS FOR GRID SPACERS GRP $8^{\circ} \quad 329300$

*5H NU $=15,5 X$. SURFACES

GRP 3 \% 329500

670 FORMAT I/1X,50H EXTERNAL CONVERGENCE CRITER TA NOI SATISFIED AFTER 329600

- I 4, IIH ITERATIONSI

680 FORMAT (10I5)

329700
RMAT (10I5)

FORMAT 1/1X,24H * ERROR IN PROGRAM, A6,:7H CALCULATION FOR,19 329900

- H THIS CASE STOPPED.,314H * *11 330000

710 FORMAT 11X,47H * ABNORMAL EXIT. MAX TIAE EXCEEOED. * $* 1330200$

720 FORMAT I//X,4IH SPECIFIED NUMBER OF TIME STEPS COMPLETEOI

740 FORMAT $1 \%$ TOTAL ELAPSED TIME IS $14,1 \times 8$ TSECCNOS END

330500

aHOG

- conro o.

EPRT,S COBRAACF TL. CONROD

FURPUR 28RI GA-B E35 S74T11 05/26/81 17:36:55 
COBRA $\triangle C F T L 111$. CONFOD

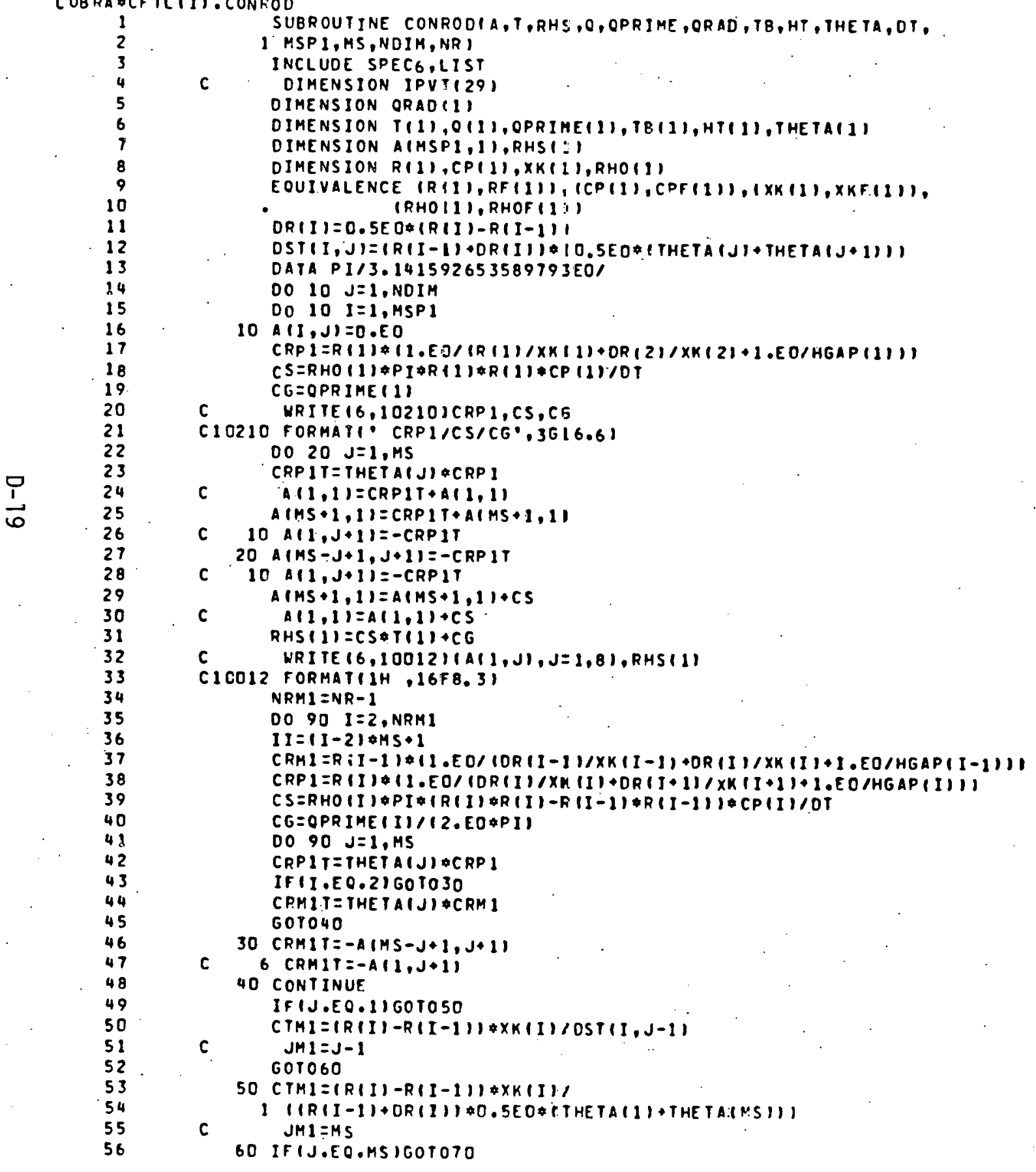


CTPI $=(R I I)-R(I-1) * \times K(I) / O S T(I, J)$ JPI $=0$

C. JPI $\quad$ JPI $1=101$ Goto80

70 CTPI $=(R(I)-R(I-1)+\times K(I)$,

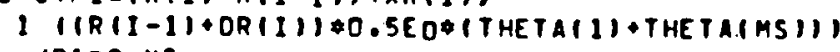
$J P 1=2-M S$ JM $1=0$

100 CIPI $=(R(N R)-R(N R-1)) * \times K(N R) /(1 R(N R-1)$. ( CRINR) I $\$ 0.5 E O$ (THETA (1) + THETAIMSII)

$120 C T M I=(R(N R)-R(N R-1)) * X K(N R) /(|R| N R-1)+$

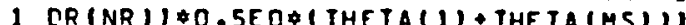


$A(I]+J, I I+J H 1)=-C T M I$

AIII.J,II+J-MSI $=-$ CPMIT RHSIII+J)=CST*TIII+J)+CGT+CRPIT*TBIJI

C FUDGE RADIANT HEAT TRANSFER AS IF HEAT SOURCE FOR INTERIM RHS $(I I+J)=R H S(I I+J)-O R A D(J)$

NEED TO CHECK ON SIGN CONVENTION FOR ORAD

C ISUBTRACT IF RADIANT HEAT LEAVES SURFACE)

C JJJ $=M A \times O I I, I I+J-M S I$

C JJJJIJJJ+14-J

WRITE $(6,10012)(A \mid I I+J, J-1, J J=J J J, J J J J), R H S I I I+J)$

140 CONTINUE

C MRIIE $(6,10313)(1 A(1, J), J=1, M S P 1), J=1, N D I M)$

C1C313 FOR YATIIH, $2918 F 13.3,111$

NMAX $=(N R-1)+M S+1$

CALL SPBFA (A, MSPI, NMAX, MS, INFO)

C CAL L DECOMPINJIM, NMAX, A, COND, IPVT, WORK,

IF I Y YF O.NE . OIHRI TE $(6,10010)$ INFO

C IFICOND.GE.1.EOIURITE 16.10013 ICONO

CIOO13 FORMATI: CONDITION NUMBER , G13.61

CALL SPBSL (A, MSP I, NMAX, MS,RHS)

C CALL SOLVEINOIM,NMAX,A.RHS,IPVTI

DO 150 JEI, NMAK

150 TIJIVRHS J)

DO $160 \mathrm{~J}=1, \mathrm{MS}$

C DETERMINE IF SURFACE OR LINEAR HEAT FLUX IS DESIRED

IS $=(N R-2) \div M 5 \cdot 1+J$

160 OIJI=(TIIS)-TB(J),

C 40 O(J)=(T(INR-2)\&MS+13J)-TB(J)I*RINRI*THETAIJI,

1 IC.SEO (RINR)-R (NR-1) I/KK(NR) + I.EO/HTIJ) RE TURN

10010 FORMATI' INFO, 15 I END

AHOG

- c U V V e.

DPRT,S COBRAACFTL.CURVE

FURPUR 2BR1 GA-B E35 S74T11 05/26/81 17:36:56

GA-MARB 1 
COBRA CFIL 11$)$. CURVE

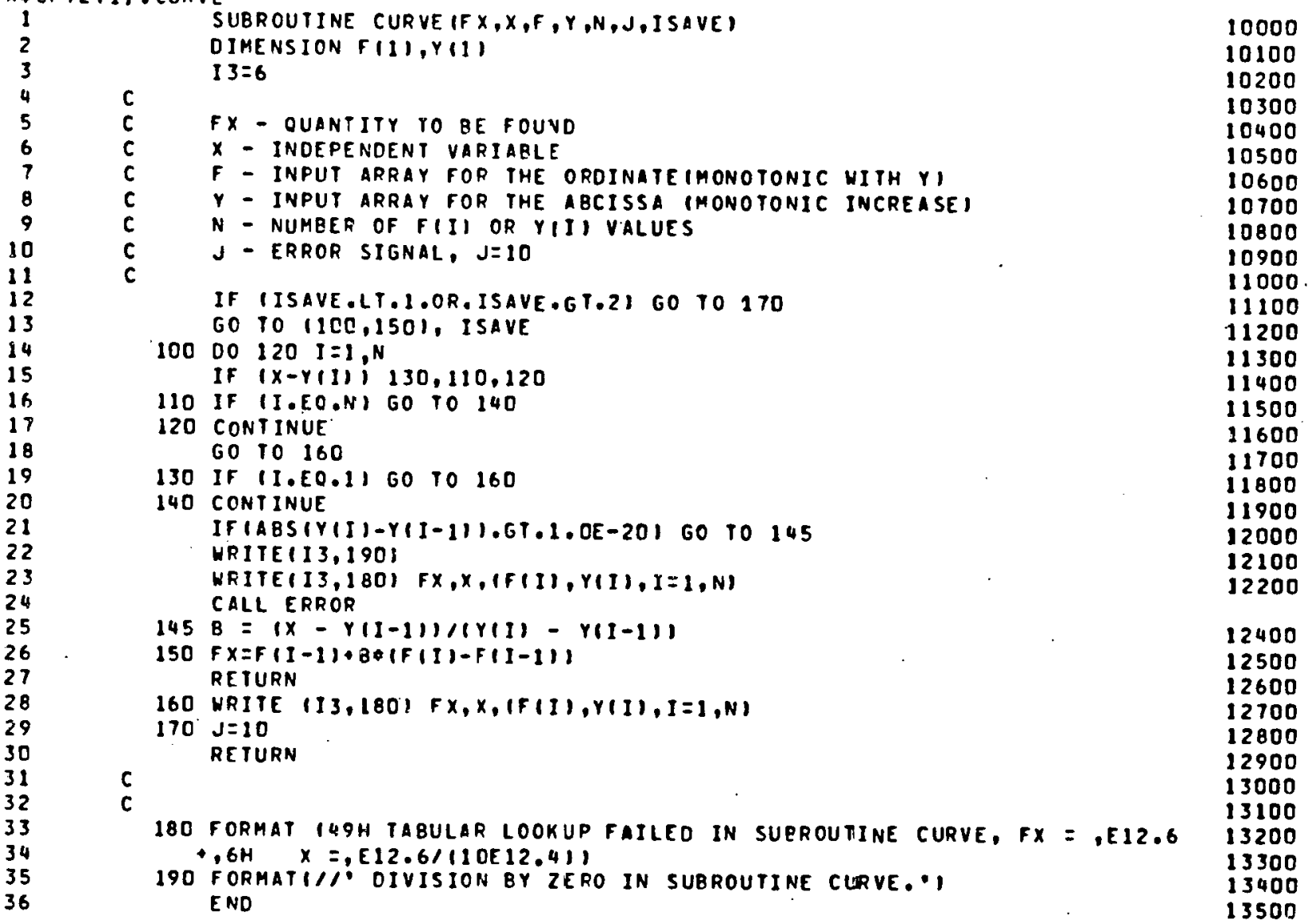

AHOG 
COBRA

1
2
3
4
5
6
7
8
9
10
11
12
13
14
15
16
17
18
19
20
21
22
23
24
25
26
27
28
29
30
31
32
33
34
35
36
37
38
39
40
41
42
43
44
45
46
47
48
49
50
51
52
53
54
55
56

56

SUBROUTINE DIFFERIIPART, J,JXI

INCLUDE SPECI,LIST

INCLUDE SPEC2,LIST

INICL UDE SPEC3,LIST

INCLUDE SFEC5,LIST

INCLUDE SPECIO,LIST

INCLUDE SPEC19,L IST

INCLUDE SPEC51,LIST

INCLUDE SPECS2,LIST

INCLUDE SFEC53,LIST

INCLUDE SPEC56.LIST

INCLUDE SPEC57,LIST

INCLUDE SPEC71 LIST

INCLUDE SFEC72,LIST

INCLUDE SFEC73, IST

INCLUDE SFEC76,LIST

INCLUDE SFECT7,LIST

LOGICAL GFIO

REAL KPOUI

REAL IDTGC, LENGTH, KIJ, LENSHO, LENROU

$S(K, I)=A M]$ NOI,$I A B S(I-J K(K)))$-AMINOII,IABSII-IK(K)I)

361800

$J M 1=j-1+1 i j \mathrm{X}$

JPRM $=(j-1)$ MNAR

IF IIPART.LT.1.OR.IPARI.GT.4I GO TO 480

GO TO $(100,270,330,450)$, JPART

C

PART 1, CHLCULATE DH/OX FOR STEADY STATE AT X ANO. T.

100 CONTINUE

DO $140 I=I, N C H A N L$

$H H=H(1, J)$

VIN $=$ HH

CALL CURVE (VOUT,VIN,KPOUT,HGPT,NPROP, IERROR, 1)

CONIIIISVOUT

CALL CURVE (VOUT,VIN,TPOUT,HGPI,NPROP, IERROR, 2 )

III $=$ VOUT

CALL CURVE (VOUT, VIN, CPPCUT, HGPT, NPROP, IERROR, 2 )

CPIII = YOUT

TPH (I) $=T(B)-H(I, D) \angle C P(I)$

140 CONTINUE

IF IIERROR.GT.11 GO TO 4 EO

If INWK.LE.OI GO TO 160

DO $150 K=1$, NHK

$I I=I K H|K|$

$J j=J K H(K)$

UWALL $(1, K)=1 . /(R$ RALL $11, K 1+1 . / H F I L M(1) 1)$

UWALL $(2, K)=1$. I IRWALL $(2, K)+1, /$ HFILMIJJ)

UWCP $(1, K)=U W A L L(1, K), C P(: I)$

UWCP $(2, K)=U H A L L(2, K) / C P(J)$

150 CONTINUE

160. CONTINUE

IF $(J 5, E 0.0) 60 \quad T 0166$

$00165 \mathrm{~K}=1, \mathrm{NK}$

$I I=I K \mid K)$

$J J=J K I K$ I

362000

362300

362500

362800

362900

363000

363100

363200

363300

363600

365100

365200

365300

365400

365600

365600

365700

365800

365900

366300

366700

366800

366900

366900

367000

367100

367200

367300

367400

367500

367600

367600

367700

367800

367900

165 CONK $(K)=(C O N(I)+C O N(J J) I \$ .5$ SAP(K,J)/LENGTHIK) OGK 
166 CONTINUE

OO $170 \quad I=1$, NCHANL

368200

CONII $=C O N(I) / C P(I)$

368300

368400

368500

170 DHDX (I) $=$ QPRIM(I)

IF (NK.EO.O) GO TO 240

369600

DO $230 \mathrm{~K}=1$, NK

369600

369700

$j J=j k|k|$

369800
369900

I $W=I F I X(S I G N(1, \ldots, N, J))$

369900

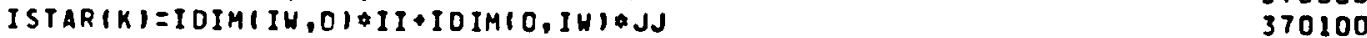

IF(J5.NE, Q) WPP(K)=WP(K)-CONK $(K)+(I I I I)-T(J J) I /(H(J J, J)-H(I I, J) \quad 370200$

1 CONTINUE 6

240 CONTINUE

c

$\begin{array}{llll}G 0 & 10 \quad 470 & 372000\end{array}$

C

$27000^{2} 20^{\circ} K=1$, NK

$J J=J K(K)$

DUMY $=G A P(K, J)$

RSTAR=RHOIII, JI

IFIK(K,J),LT.O.IRSTAR=RHOIJJ:,JI

$W P(K)=G C * R S T A R \# D U H Y \otimes D U M Y$

$280 \operatorname{BETA}(K, J)=0$.

372800

372900

IF $(K 12, E Q .0) \quad 60$ TO 310

DO $300 \mathrm{KK}=1$, NK

$I I I=I K(K K)$

JJJ $=J K(K K)$

SAVE $=0$.

IENO $=$ LOCA $(N A A A P I, K K)$

DO $290 L=1, I E N D$

$K=L O C A(L, K K)$

$W W=W(K, J)$

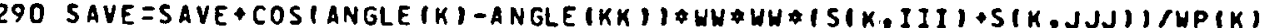

300 BETA $(K K, J)=S A V E$

$31000320 K=1, N K$ 320 CIJ (K) $=A M A X I 1.001, A B S(W(K, J) J 1 \% K I J 0.5 / W P(K)$
REIURN

C PART 3 , CALCULATE DP/DX HITHOUTH

330 CONTINUE

350 IF I.NOT.GRIOI GO 10370

IF (NRAMP.LE.OS) GO TO 480

OUMY = AMINI I , ,FLOATIITERATI/FLOATINRAMP II

IF IOT.LT.1.EG) DUMY $=1$.

DO $360 \quad I=1, N C H A N L$

COI = COII, NGTYPEI *COCIINGTYOEI

- ICOCZINGTYPEI + CDC3INGTYPEIAR

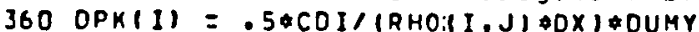

370 DO $380 \quad 1=1$, NCHANL

AIJI $=1 . / A(I, J)$

$A P P I=1, / A P P \mid I, J I$

$F M]=F(1, J M 1)$

OPK(I)=IDPKIII+.5\$FSPIII\#VIII/OHYOII,J) \#AIJI\#AIJI

373000

373100

373200

373300

373400

373500

373600

373700

373900

374000

374100

374200

374300

374400

374500

374600

374700

374800

374900

375000

375100

375200

375300

375400

375500

375500

375600

375700
375800

375900

376000 
TERMIIII $=$ SIGNIOPKII),F(I,J)

376100

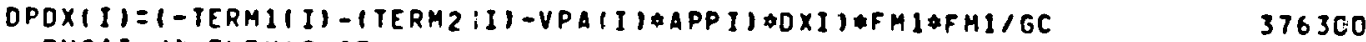

- -rHoRI JlaELEV*0.80

$380 \mathrm{FLOH}(I)=F(I, J)+F M 1$

IF (OT.GT.1.EG) 60 TO 400

DO $390 \quad I=1$, NCHANL

376500

376600

376700

RHODOT =IRTOII, JI-RHOOLDII,JII/OT

376800

OPOXII) DFOXIIJ+ (FOLDII,J)-FII,JMIII/APPII,JI/OTGC+RHODOT/GC\#10X10 376900

30 CONTINJE

400 CONTINUE

IF IFTM.LE,O..OR.NK.EQ.OI GO TO 440

377100

377200

DO $410 \quad I=:$, NCHANL

410 DPWP $(1)=0$.

DO $420 \quad K=:$, NK

$I I=I K(K)$

$J J=J K(K)$

DUMY $=(U B(T I, J)-U B(J J, J))$ WP $(K)$

DPHPIIII =OPWPIIII) DUMY

420 DPWP(JJ)=DPUP (JJ)-DUMY

DO $430 I=I$, NCHANL

430 DPDXII $=$ DPDXII)-DPUP(I)/APPII, JI/GCAFTM

440 CONTINUE

GO TO 470

PART 4, CALCULATE OP/DX WITH

450 IF IJ.EQ.1) RE IURN

DO. $460 \mathrm{~L}=1, N K$

I $I=I K(L)$

$J J=J K(L)$

L. $P R M=L+J P R M$

OPOXIIII =DPOXIIIIDWPRMILFRMI DDTIIILI/GC

377300

377400

377500

377600

377700

377800

377900

378020

378000

378100

378200

DPDX.IJJ) $=D P O X(J J)-W P R M(L F R M) * D I J J(L) / G C$

378400

378500

378600

378700

378700

378900

379100

379200

379300

379400

379500

460 CONTINUE

470 RETURN

480 IERROR $=2$

RETURN

379600

379700

379800

379900

ENO.

380000

a.HOG

- OIVERT:

APRT,S COBRA $S$ CF IL DIVERT

FURP.UR 28R1 GA-B E35 $574 T 11$ 05/26/81 17:36:56 
COBRA CFTL II, OIVERT

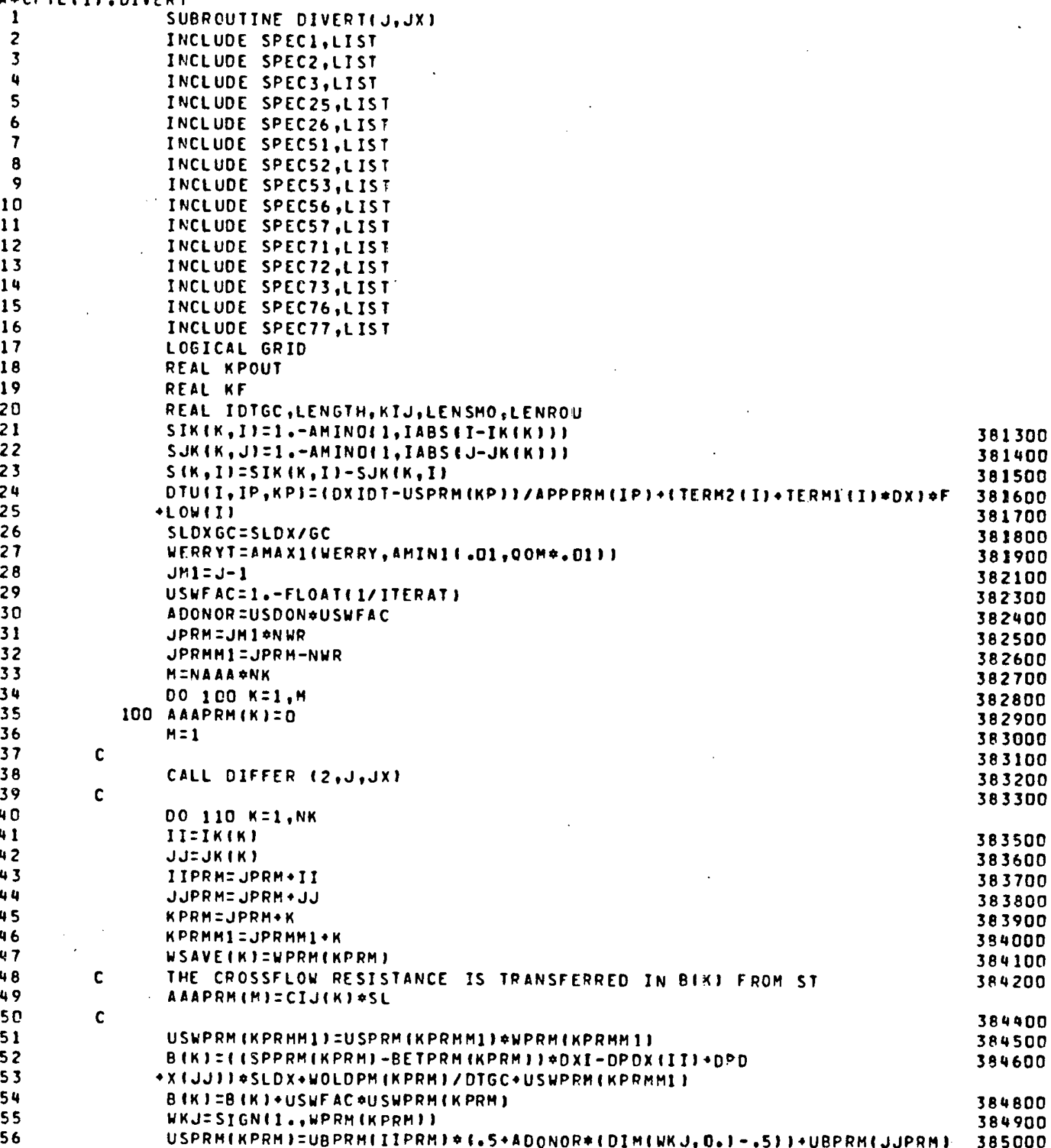


Q Q 1.5 + ADONOR* DIM10.,HKJ)-.51)

DIII KKI $=0 T U I I I, I I P R M, K P R M)$
DTJJIK) $=0 T U I J J, J J P R M, K P R M)$

USPRMIKPRM I = USPRM (KPRMI $10 \times 3 C$

385200

$A A A P R M(M)=A A A P R M(M)+11$ +USNFAC $\approx U S P R M(K P R M)+10 T G C \quad 385500$

$M=M+N A A A$

SET UP THE SIMULTANEOUS EQUATIONS

$M=0$

$N=0$

$00140 \quad K=1, N K$

AAAMAX $=0$.

NLOOP = LOCAIINAAAPI +N)

385500

385700

385800

385900

386000

$I I=I K(K)$

$J J=J K(K)$

DO $120 \mathrm{IL}=1, \mathrm{NL}$ OOP

$M P R M=M+I L$

$M P R M_{1}=N+I L$.

$L=L O C A I$ IMPRMI)

DUMMY $=(S I K I L, I I)-S I K I L, J J O) \neq D T I I I L)-(S J K I L, I I 1-S J K(L, J J I) \div D T J J(L)$

AAAPRM (MPRM) = DUMMY \$SLDXGC PAAAPRM (MPRM)

A AAMAX =AMAXILIABS (AAAPRM (M DRM)) OAAMAX)

120 CONTINUE

If IAAAMAX EO.ABSIAAAPRM(M+1)) GO TO 130

IERR OR $=3$

HRITE(I3,291) (IAAAIL,LL),L=1,NAAA),B(LL),LL=L,NK)

c

40 CONTINUE

PFE-INITIALIZATION OF W

IFIITERAT,GT. I OOR. OT .LT. I.E8) 60 TO 160

DO $150 \cdot k=1, N K$

$150 H(K, J)=H(K, J)+F O I V(K)+H(K, J M I)(1,-F O I V(K))$

386200

386300

386400

386500

386600

386700

386800

306800

387000

387200

387300

387400

387500

387800

387900

388000

388100

388200

388300

388400

388500

388600

388600

388700

388800

388900

389000

389100

NF $=0$

$N B A C K=N K+1$

$M=D$

DO $180 \quad K=1, N K$

$K M 1=K-1$

$M=K M 1 * N A A A$

$N=K M I * N A A A P I$

ANORM $=A C C E L Y / A A A P R M(M+11$

389200

389300

389400

389500

38500

389600

399700

389800

389900

390000

NLOOP.=LOCA I IN+NAAAPI)

DO $170 \mathrm{~L}=1$, NLOOP

390100

390200

390200

390300

IF $A D D=I F I X(1,-F O 1 V(K))$

IBAOD = IFIXIFDIV(K))

390400

$N F=N F+I F A D O$

NBACK $=N E A C K-I B A O D$

390600 
* DIVERT* PERFORM ITERATIVE SOLUTION IGAUSS-SEIDEL H: TH OVER-RELAXATIONI DO $210 \quad I=1$, ITRY ISAVE $=1$

OUAN $=0$.

DO $200 K H=1, N F$

$K=\operatorname{MARK}(K W, 1)$

$C=B(K)$

$K M I=K-1$

$M=K M I \times N A A A$

$N=K M I * N A A A P I$

IEND $=L O C A I$ IN + NAAAP II

DO $190 \quad I L=1$, IEND

$L=L O C A \perp(N+I L)$

$190 C=C-A R A P R M(M+I L)$ QHPRMIL+JPRM)

SOLVE FOR CROSSFLOWS IF NO. FORCED DIVERSION KPRM $=K+J P R M$

WPRM $(K P R M)=W P R M(K P R M)+C$

ERROR = ABS $(C) /(A B S($ WPRM $(K P R M))+1, E-6)$

OUAN = AMAXI IOUAN,ERROR I

200 CONTINUE

CHECK INTERNAL CONVERGENCE

OIM = AMAXI IOIM, QUAN )

IF (ISAVE.LTOITRYM) GO TO 210

210 CONTINUE

220 CONTINUE

ISAVTEISAVT+ISAVE

IF $(J X, N E$. NOXPI) $60 \pi 0240$

$c$

DO $230 \mathrm{~K}=1$, NK

230 USW K, J) $=U S(K, J) \& W(K, J)$

240 CONTINUE

IF IICOUNT.EQ.01 GO TO 250

IF (MODIITERAT, 4). NE.1) GO TO 270

250 WBAR $(J X)=0$.

DO $260 \mathrm{~K}=1, \mathrm{NK}$

260 WBAR $(J X)=W B A R(J X)+A B S(W(K, J))$

WBAR $(J X)=$ WBAR $(J X) / F L O A T(N K)$

270 CCNTINUE

IERROR $=$ ?

QUAN $=0$.

OO $280 K=1, N F$

$K P R M=J P R M+K$

$A B H=A B S$ (HPRMIKPRMI I+1,

ODUM $=($ HBAR $(J X) / A B H)+2 \% .001+18 H$

QUAN = AMAXI (ABS I (UPRM (KPRM) -WSAVE (K) / /ODUM), OUAN

390900

391000

391100

391200

391300

391400

391500

391700

391800

391900

302000

392100

392200

392300

392400

392500

392600

392700

392900

393000

393100

393200

393300

393400

393500

393600

393700

393800

393900

394000

394100

394200

394300

394400

394500

394500

394600

394700

394800

394900

395000

395100

395400

395600

395700

395800

395900

396000

396100

396200

396300

396300

396400

396500 
- D IVERT:

DATE 052681

291 FORMAT I4BHILOSS OF OIAGONAL DOMINANCE IN SUBROIJINE DIVERT/25X,24 - HCOEFFICIENT MATRIX (AAA),30X,23HFORCING FUNCTION 131/15X,5F10.3,3 397000 $.0 \times, F 20.311$

397100 174 END

397200

2พOG

* D U T

APRT,S COBRAOCFTL.DU:T

FURPUR 28R1 GA-B E35 574T111 O5/2E/81 17:36:57 
COBRA OCF TLIII.OUCT

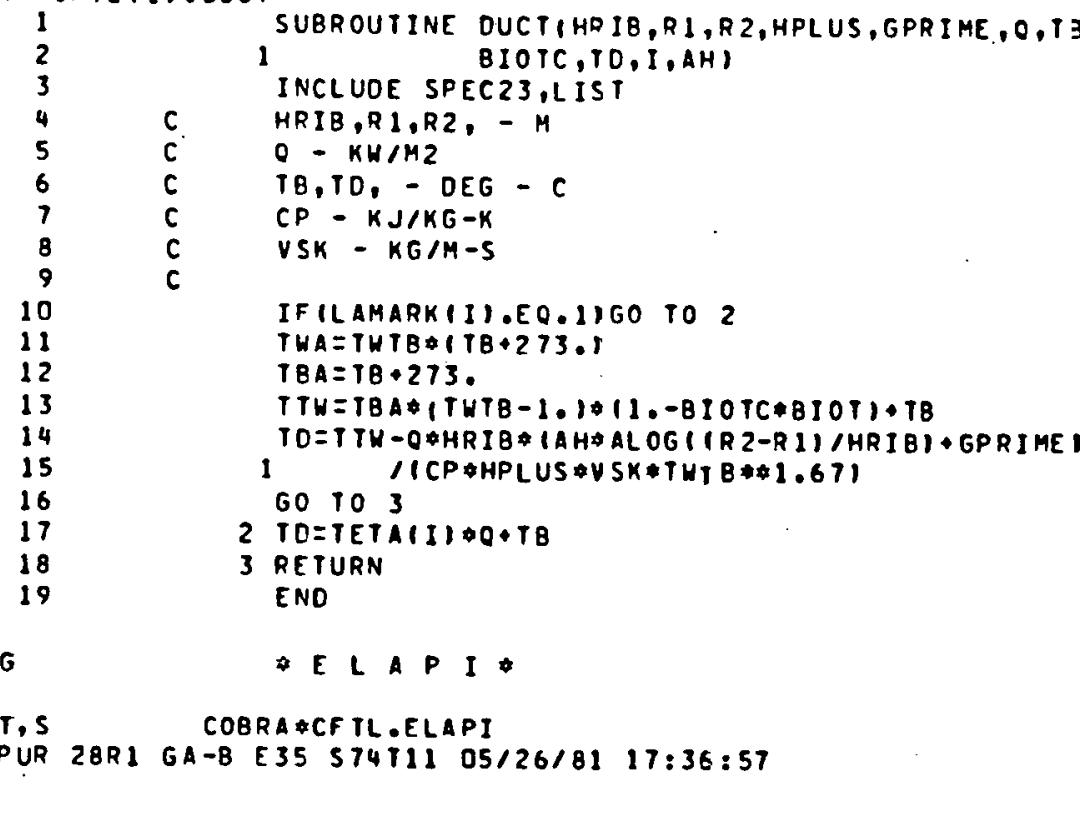


- E l a I I

COBRA OCFIL I I).ELAPI

$\begin{array}{lll}1 & \text { SUBROUTINE ELAPI } & \\ 2 & \text { CALL TICKERITII } & \text { GA-MARBI } \\ 3 & \text { RETURN } & \\ 4 & \text { ENTRY ELAPIMTIMEI } & \\ 5 & \text { CALL TIEKERITII } & \text { GA-MARBI } \\ 6 & \text { MTIMEITI-TOIJ60..1. } & \end{array}$

aHOG

$G A-M A R B I$

DPRT, $S$

- er r o r.

FURPUR 28RI GA-B E35 S74T!1 O5/26/81 17:36:57

$\stackrel{\stackrel{1}{\omega}}{\omega}$ 
COBRA\&CFIL11) ERROR

$\begin{array}{ll}1 & \text { SUBROUTINE ERROR } \\ 2 & \text { CALL MERR } \\ 4 & \text { RETURN } \\ & \text { END } \\ & \text { F ORCE }\end{array}$

DPRT, S COBPA $\$$ CFTL .FORCE

FURPUR 28R1 GA-B E35 S74T11 05/26/81 17:36:58 
CCBRA \#CFIL 11 . FORCE

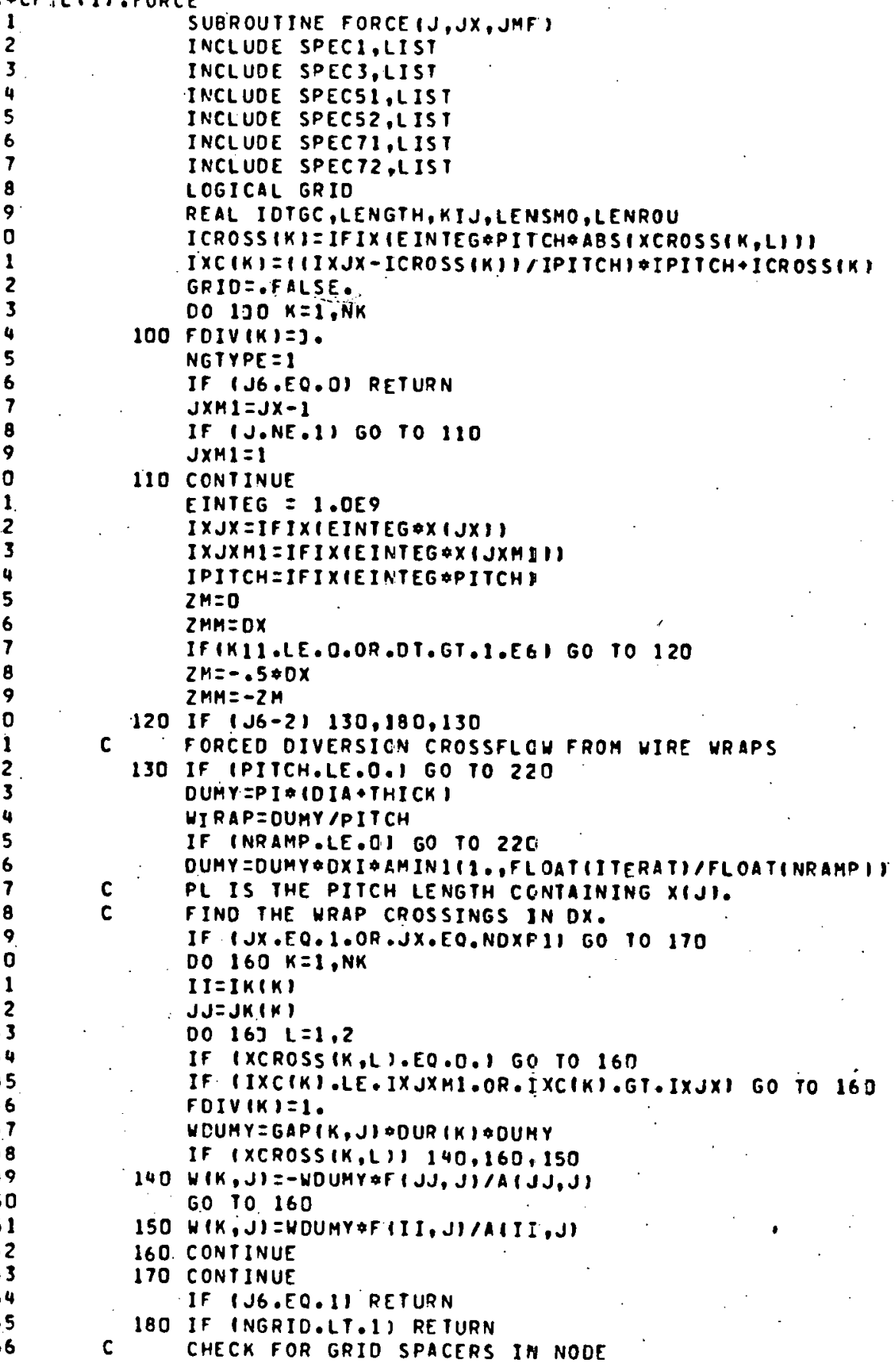

409900 410300

410500 410600 410700 410800 410900 411100 411200 411400 411500 411600 11700 4.11800 411900 412000 412100 412200 412300 412300 412400 412500 412600 412700 412800 412900 413000 413100 413100 413200 413300 413400 413500 413600 413700 413800 413900

414100 414200 414300 414400 414500 414600 414700 414700 414800
414900 


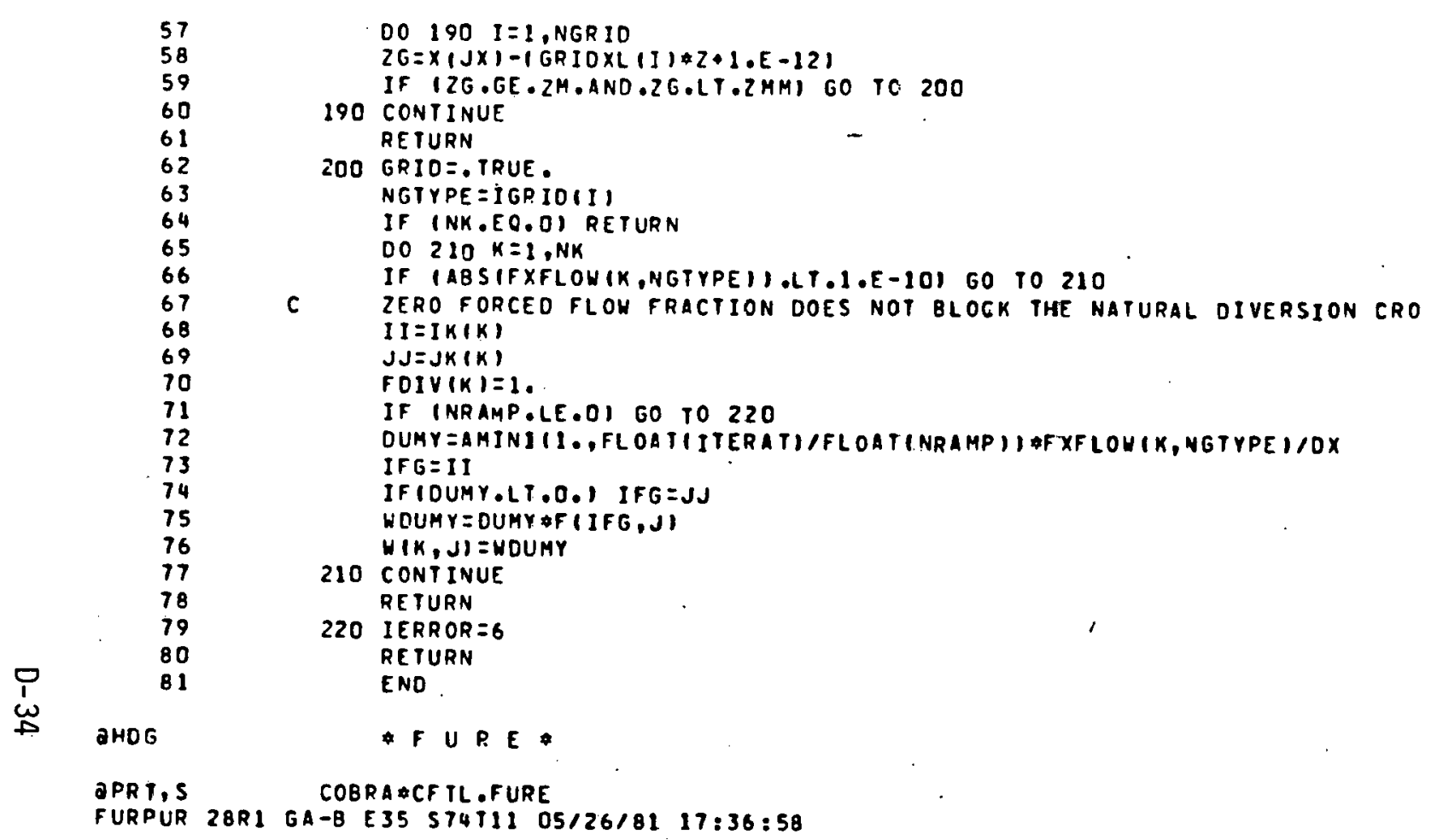

415000

415100

415200

415300

415400

415500

415600

415700

415800

415900

416000

416100

416200

416300

416400

416500

416600

416700
416800

416900

417000

417100

417200

41.7300

417400 
- FUFEE

DATE 052681

COBRA $\$ C F T L 11)$.FURE

1
2
3
4
5
6
7
8
9
10
11
12
13
14
15
16
17
18
19
20
21
22
23
24
25
26

FUNCTION F'JRE IREI

C \# RE:BUNGSKOEFFIZIENT NACH PRANDTL-NIKURAOSE \#*

19900

20000 FUNIF: = .0*ALCGIOIRE WSOFTIFII-0.4-1.0/SORTIF) $J=1$

$F R=0.1 * R E * 1-0.251$

$F R R=0.07 * R E \$ 1-0.251$

$R=$ FUN(FR)

$R R=$ FUN(FRR)

$1 F R 2=F R-R Q \mid F R R-F R I / I R R-R$

$J=J+1$

IFIJ.3T.301 60 TO

$R 2=$ =UNIFR2I

IF (ABSIRZI.LT , 1. OE - 4 ) 60 TO

IF(IR-R2).GT.0.0) GO TO 2

$F P R=F R$

$F R R=F R 2$

$G O T O T$
$R=R 2$

$F R=F R 2$

GO TC 1

4 FURE $=F R 2$

RE TUFN

20100

20200

20300

20400

20500

20600

20700

20800

20900

20900

21000

21100

21200

21300

21400

21500

21600

21700

1800

21800

21900

22000

5 WPITE $(6,10)$

22100

FORMATI/10X,25HREITERATION FAILED $J=$, I2I
RETUFN END

AHOG

- HEAT:

DPRT,S COBRAACFTL.HEAT

FURPUR 28R1 GA-8 E3E ST4TII 05/26/81 17:36:58 
COBRA $*$ CFTLII), HEAT

SUBROUTINE HEATINSS, J8, HHT,JX3)

INCLUDE SPECI,LIST

INCLUDE SPECG,LIST

INCLUDE SPEC12,LISI

INCLUDE SPECI9,LIST

INCLUDE SPEC22,L IST

REAL IOTGC,LENGTH, KI J,LENSHO,LENROU

c

CALCULATE THE HEAT INPUT TO EACH SUBChANNEL AT POSITION J.

433600

$\mathrm{J}=\mathrm{JB}$

$J X=J \times 3$

THIS SUBROUTINE CONSISTS OF THREE PARTS AS ENOICRTEO BY NHT

PART 1 - INITIALIZES ALL TEMPERATURES, COMPOTES ROD POWER;

ROD AND CLAD TEMPERATURES AND SURFACE HEAT FLOX.

PART 2 - CALCULATES HEAT INPUT TO INOIVIDUAL SUBCHANNELS

PART 3 - REMOVED

RODPWR = AFLUX $\$ P O W E R$

IF INHT,EO,2) 60 TO 170

IF IITERAT.GT.1) RETURN

IMPLICIT SOLUTION REQUIRES ITERATION FOR ROC TEMPERATURES

434900

435000

435100

435200

435300

435400

435500

435800

436100

437700

437800

DO $140 \mathrm{JF}=1$, NDXP 1

IF IJF.E0.1160 T0 140

$D X=x(J F)-x(J F-1)$

$0 \times 2=0 \times 0 x$

$J X=J F$

c

$J=J F$

C CALCULATE AXIAL POHER FACTOR

THE FOLLOHING SET OF STATEMENTS PROVIDES AN

ACCURATE DETERMINATION OF AXIAL HEAT FLUX IN NODES WHERE STEP CHANGES OCCUR.

CALL CURVE (OAX $1, X(J X-1) / 2, A X I A L, Y, N A X, I E R R O R, 1)$

CALL CURVE I2AX2, XIJXIIZ, AXIAL, Y, NAX,IERROR, I

TEST $=(0 A \times 2+1 . E-5)(10 A \times 1+1 . E-5)$

IFITEST.LT.3..AND. TEST.GT.0.331GO TO 15

C ENDPOINTS HALVED ACCOROING TO TRAPEZOID RULE

OAXSUM $=10 A \times 1+O A \times 21 * 0.5$

OXF $=0 \times 120.0$

$0 \times F=x=X X 120.0$
$X F-11$

$X F=x \mid J X-11$
0011 IDXF $=1,19$

$X F=X F+O X F$

CALL CURYE IOAX,XF/Z, AXIAL,Y,NAX, IEFROR, 11

11 OAXSUM $=$ OAXSUM + OAX

OAX $=$ OAXSUM 20.0

GO 1018

15 CONTINUE

XOUMY =AMAX $1(1 X(J X)-.5 * D X)(2,0.1$

CALL CURVE IOAX, XDUMY, AXIAL,Y,NAX, IERROR, 11

437900

438400

438600

438700

438800

439200

439300

439400

439700

439800

439900

440000

440100

440200

440500

440600

440700

440800

440900

441000

441100

441100

441300

441400

441500

441600

441700 
DOOD R DOPHR:OOAX

C CALCULATE THE FORCEO HEAT FLUX FROH EACH ROD FLUX $(N, J)=F O D O \# R A O I A L(N)$

130 CONTINUE

140 CONTINUE

c

C. PART 2 - ChLCULATE HEAT INOUT TO EACH CHANNEL
170 DO 180 I $=1$,NCHANL

441800

441900

$44200 \mathrm{C}$

442100

444500

444600

444600

445700

445800

445900

446000

IBO OPRIM(I) $=0$.

$M T=0$

DO $22 \mathrm{E} N=1, N R O D$

446200

$F L=F L L X(N, J)$

DO $210 \quad L=1 . M S C$

$I=L R(N, L)$

446300

446703

446800

446900

447100

IFIIFL NE II GO

$M T=M T+1$

447200

210 OPRIMIII =OPRIMIII+FL*PHI IT,LI*PI*OIN

220 CONTIHUE

RE TURN

451300

END

453400

APRT,S COBPA $\$$ CF IL.HEL IUM

FURPUR 2QR1 GA-B E35 ST4T11 O5/26/81. I7:36:59 
COBRA OCF ILI11. HELIUY.

SUBROUTINE HELIUMIPRESSI

INCLUDE SPEC5,LIST

REAL KPOUT

THIS SUBROUTINE CALCULATES THE PROPERTIES CF HELIUM GAS AS A 456500

456400

FUNCTION OF PRESSURE AND TEMPERATURE. THE PROCEDURE ANO

NOMENCLATURE ARE SIMILAR TO SUBROUTINE STEAM IN COBRA4.

ROUTINE WRITTEN ON $3 / 7 / 77$.

PRESS = PRESSURE |PAI.

HENTITEMP,PRESI = 5557.J + 5193.0*TEMP + PRES*19.489433E-4

$19.528079 E-4 / 01+2.739 \$ 7 E-3 / 02$ - TEMP\$13.259251E-5/01*\$2 -

$22.5776 E-6 / 02 * 211$

PS = PRESS

ISTAG $=3$

IF INPROP.LT.5I ISTAG = I

MPMI = NPROP - ISTAG

DT $=$ DTMAX - TF

DELTT = OT/FLOAT (MPMI)

DO $201=1$, NPROP

FACT = FLOATII - ISTAGI

IF II.LE.ISTAG) FACT = FLOATII - II/FLOATIISTAGI

TS = TF DELTTHFACT

$T S=T S \cdot 273.15$

COMPUTE HELIUH ENTHALPY I HENT, KJ/KG

$01=1 \cdot 0-3 \cdot 42068 E-2 * T 5$

$O Z=1.0+9.40912 E-4 * T S$

HENTH = HENTITS,PSI/1000.

COMPUTE HELIUM CONDUCTIVITY I HCON, KWIM-K

456600
456700

456800

456900

457000

457200

457500

457600

457700

457900

458000

457790

458200

458300

458400

458500

458600

458700

458900

459100

459200

459300

459500

459700

$459800^{\circ}$

HCON $=2.774 E-3 * T S * 0.701$

HCON $=H C O N / 1000$.

460000

460200

460300

HVIS $=3.953 E-7 * T S \$ 0.687$

COMPUTE HELJUM SPECIFIC VOLUME, HVOL I CU MIKG

HVOL $=2077.22 * 15 / P S+9.489433 E-4+9.528079 . E-4 / 01+2.73947 E-3 / 02$

COMPUTE HELIUM SPECIFIC HEAT (HCP) K J/KG-K

$T S 1=T S-1.0$
$T S 2=T S+1.0$

DELTAT $=2$.

$D I=1.0-3.42068 E-2 * 151$

$02=1.0+9.40912 E-4 * T S$

HENTI = HENTITS1, PS I

OI $=1 . \mathrm{C}-3.42068 \mathrm{E}-24 \mathrm{TS}$

461300

461400 461500

461700 461900 461900 462000 
* He L I U M *

$02=1.0+9.40912 E-4 * 152$

HENT2 = HEMTITS2,DSI

c

HCP

462200

46.2400

$\begin{array}{ll}\text { HGPTITI }=\text { MENTH } & 462600 \\ \text { TPOUTIII }=\text { TS-273.15 } & \end{array}$

TPOUTIII $=$ TS-273.15

KPOUTII = HCON

UPOUT(1) = HVIS

VPOUTIII = HVOL

CPPOUI(I):= HCP

20 CONTIMUE

$C T=-$. ?

RETURN?

END

463000

463100

463200

463209

463300

463409

- I O D T

DHDG

COBRA CF TL .IDDT

DPR T , $S$

FUPPUR 28RI GA-BE35 S7AIII D5/26/81 17:36:59 
COBRA CFIL11). 10OT

NRROUINE IOOTIH, RE, R1,R 2, TWTB, XLAM, ST, I, JN

INCLUDE SPECI4,LIST

INCLUDE SPEC15,LIST

INCLUDE SPECI7,LIST

INCLUDE SPEC24,LIST

LOGICAL LF

105100

LOGICAL LFEFF , LREFST

105300

FSAVE, SSAVE - INITIAL GUESS FOR EACH NODE DOSITION

$X$ LAMST $=4 . * F U R E$ (RE)

FSAVE $=2.0 \times$ XAMST

STST $=.003$

SSAVE $=.010$

$X L A M=X$ LAMST

156100

$S T=S T S T$

LREFF = TRUE.

IFITUTB : IT. 1,1,THTB $=1$.

IFITUTB $A T$

106200

$A L 2=A L \otimes A L$

108500

$A H S=C G(3)$
$A R=C R(2)$

LF =.FALSE.

IHS $=0$

DO 1000 ITER $=1,50$

$x A=2.5$

$F=X L A M / 4$.

$J J=1$

300 CALL BEFIAL,RE,F, XA,2,50

CALL BETAF IBET, AL, C2COII

$B E 2=B E T \circ B E T$

CALL BEF 4 (FF 1,FF 2, RE 1, RE 2 I

OFI = SQRTI2/FFII

FR=FURE IRE2

$F 12=F F 1 / F F 2$

$F 2 S=F R+1.005 * F 12 * 1.0561$

WF $2=S O R T I F 2 S / 2$.

$X A A=(5.5-1 / H F 2) /(13+B E T) / 11+B E T) / 2-A L O G(R E 2 * H F 2 / 11+B E T) / 2)$

IF $(A B S(X A-X A A) \cdot L T . .100$ I GO TO 550

$X A=X A A$

$J J=J J \cdot 1$

IFIJJ.GT. 20160 TO 555

60 10 300

555 URITE $(6,556)$

556 FORMAT I3OX, -AS ITERATION FAILED'I

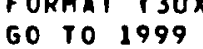

$550 \times A=X A A$

REIWIRE I*TWTB*(-1.67)

HPLUS $=H / 2 . / R 1$ \&E1W//BEZ/ALZ-1 -1/OFI

$A P=C R(2)$

$H O L=1 B E T \& R 2-R 11 / H$

IFIHPLUS.GT.CRI3)1GO 10101

$A R=C R(6)-A L O G(H P L U S)+(C R(7)-C R(B) * A L O G(H P L U S)$

GIHS $=2.50+11.50+0.50 * 8 E T / A L / / 11 .+B E T / A L$,

RHPHS $=O F 1+G 1 H S-2.50 * A L O G(H D L)$

$101 G 1=A R * 11.50+0.50 * B E T / A L / / 11 .+B E T / A L$

109500

109600

109700

110300

110700

110900

111000

111100

111200

111300

111400

111600

111700

111800

111900

112000

112100

112200

112300

112600 
RHP $=O F 1+G I-A R+A L O G(H D L)$

IFI.NOT. LFIGO TO. 1999

IF IST) $1999,1999,560$

560 GHP = SORT ( BE 2-AL 2)/AL/11,-AL) //ST*SORI (XLAM/8.I-AHS

I $\# A L O G(11-A L) * R 2 / H)+A H S *(0.5 \cdot 1.5 * A L) / 11 .+A L)$

1999 CONTINUE

IFILF) GO 10710

RPRIME = CRI1)

IFIHPLUS.GT.CR 31$)$ GO TO 551

RPR I ME $=C R(4)-C R(5)$ \& L OG (H DLUS)

RHSH $=5.5+2.5 * A L O G(H P L$ JS $*$ THTB $* 1.67)$

TEST $=R P R I M E+(A R-2.50) *(A L$ OG IHOL) $-.5-1.1(2 .+H O L A H / R 1)$ )

$A L O C K=T E S T-R H S M$

IFIIHS.EO.1.AND.ALOCK.GT.-.15)GO TO 100

IHS $=0$

C

IF TEST, RHSM FLON IS HYDRAULICALLY SHOOTH

IF (ALOCK.LE.O) GO TO 561

100 IHS $=1$

561 IFI.NOT. LREFFI GO TO 705

ROIF = RHP - RPRIHE

ROIFHS = RHPHS - RHS

LREFF = .FALSE.

$X L A M=F$ SAVE

GO TO 1003

705 CONTINUE

IFIIHS.EQ.1160 TO 102

IF (ABSIRHP-RPRIME) .LT. .05) 60 TO 700

$X L A M=$ (XLAMST IRHP-RPRIMEI - XLAHERDIF I

GO I RHP-RP RIME -RDIF

102 IF IABS IRHPHS-RHSMI.LT_.05IGO TO 700

$X L A M=(X L A M S T$ |RHPHS-RHSM) -XLAM\#ROIFHS / IRHPHS-RHSM-ROIFHS I GO 101000

700. CONTINUE

$$
\text { LF }=\text { TO TRUE. }
$$

710 CONTINUE

$G O=C G(1)$ \#PLUS $\$ C G 12)$

IFIHPLUS.LT.CG (6) IGD = CG17)

GPPIME = GOAPR\#CG (U)

IFI NOT OREFSII GO TOTIL

GOIF = GHP - GPRIME

LREFST = .FALSE.

$S T=$ SSAVE

GO $10 \quad 1000$

112 CONTINUE

IFIABS IGHP-GPRIMEI, .LT. DI GO TO 2000

$S T=$ (STST IGHP-GPRIME) - ST \$GDIF)

$1000^{\circ}$ CONTINUE

( IGHP-GPRIME -GD IF)

WRITE (6, 1) RHP, RPRIME, XLAM, GHP, GPRIME, ST, I, JN, AR, TEST, RHSM, HPLUS

1 FORMAT 1" SUBROUTINE IDDT NOT CONVERGED. RHP = .F $9.4,2 \times$, 'RPRIME $=0$

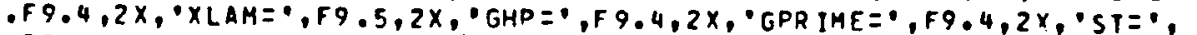

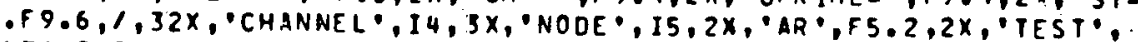

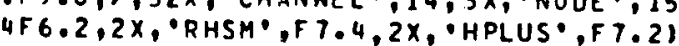

RETURN

2001 : CNTINUE

15500

115600

115700

115800

115900

1160.00

$1163.00^{\circ}$

116400

116500

116600

116700

116700

117200

117400

117700

118200

118400

118500 


\section{115 \\ CALL ERROR
2000 CONTINUE}$$
116
$$

117

118

110

119

120

121
122

HPWII, JNI $=$ HPLUS

RHPLSII,JN) =RHP

IFIIHS.EQ.0160 TO 750

RHPLS I , JN $)=$ RHPHS

AR 2.50
RETURN

END

จHDG

DPRT,S

COBRA \#CFTL.LIMITS

- L I M I I S

FURPUR 28R1 GA-B E35 S74T11 05/26/81 17:37:00
119000

119100

119200 
* LIMITS*

COBRA $\triangle$ CFTLI1).LIMITS

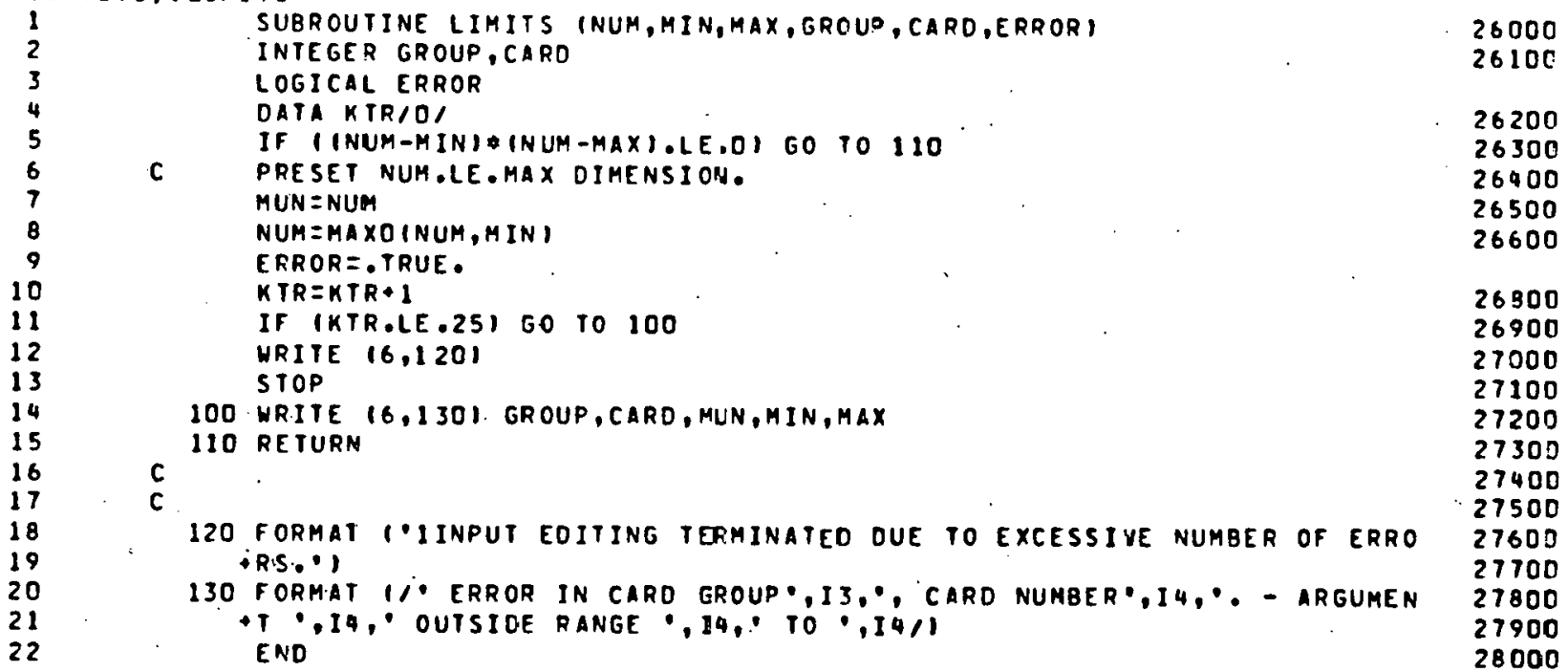

i

* L O

COBRA CF IL ILAD

F URPUR 28R I GA-BE35:S74T11 05/26/81 17:37:00 
COBRAOCFILIII. LOAD

SUBROUTINE LOAD $\mid X, Y, Z, M I N, M A X, L I M I T$, STEP, IMAGE, CARO,LU

28300

REAL ARRAY $(80), X(1), Y(1), 2111$

INTEGER LIST $(80)$, STEP, CARD, COUNT, IHAGE 12$)$

EQUIVALENCE ILISTII),ARRAYIIII

IF (MAX.LT,MIN) GO TO 120

IF $($ ISTEP- 1$)+(S T E P-3) .6 T$.0) GO TO 120

MAXKRD = (MAX-1) \& SEF/LIMIT+1

MINKRD $=$ IMIN-1 +5 TEP/LIMIT \&

CARD = CARD + HAXKRO

COUNT $=0$

$\mathrm{J}=0$

$100 \quad j=J+1$

IF IJ.GT.MAXKRDI GO TO 120

READ (LU,IHAGE) (ARRAYII) I I I, LIMIT)

IF IJ.GT.MINKRDI GO TO 100

DO 110 I $=1$,LIMIT,SIEP

COUNT $=$ COUNT 1

If (COUNT.GT.HIN) 60 TO 100

XICOUNTII=ARRAYII)

IF ISTEP.EQ.1) GO TO 110

$Y(C O U N T)=A R R A Y(I+1)$

IF ISTEP.EO.2) GO TO 110

2 (COUNT) =ARRAY $(I+2)$

110 CONTINUE

GO TO 100

120 RETURN

ENO

28700

28800

28900

29000

29100

29200

29300

29400

29500

29600

29700

29800

29800

29900

30000

30100

30200

30300

30400

30500

30600

30700

30700

30800

- M IX

30900

वHOG

COBRA HCF TL.MIX

FURPUR 28R1 GA-B E35 S74T11 05/26/81 17:37:00 


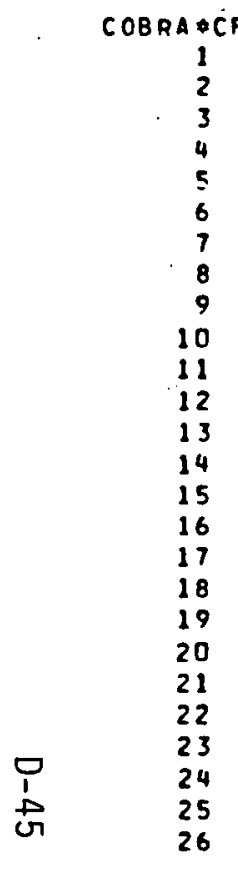

AHDG
SUBROJTINE MIX $M$ (J)

INCLUDE SPECIOLIS

INCLUIJE SPECIO.LIST

REAL IDTGC,LENGTH, KIJ,LENSMO,LENROU

DO $120 K=1, N K$

$I I=I K(K)$

$J J=J K(K)$

FRCTN $=$. E\#IFSPIIII + FSPIJJI)

$N P(K)=0$.

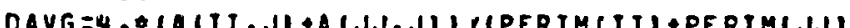

483000

483100

483200

4833010

483400

483500

483900

484000

484100

484100

484200

484300

484400

484500

484600 484700

485400

485500

485600

485700

485800

APRT,S COBRA + CFTL.MORCON

FURPUR 28R1 GA-B E35 5T4T11 05/26/81 17:37:00 
COBRA $\$$ CF ILU 11. MORCON

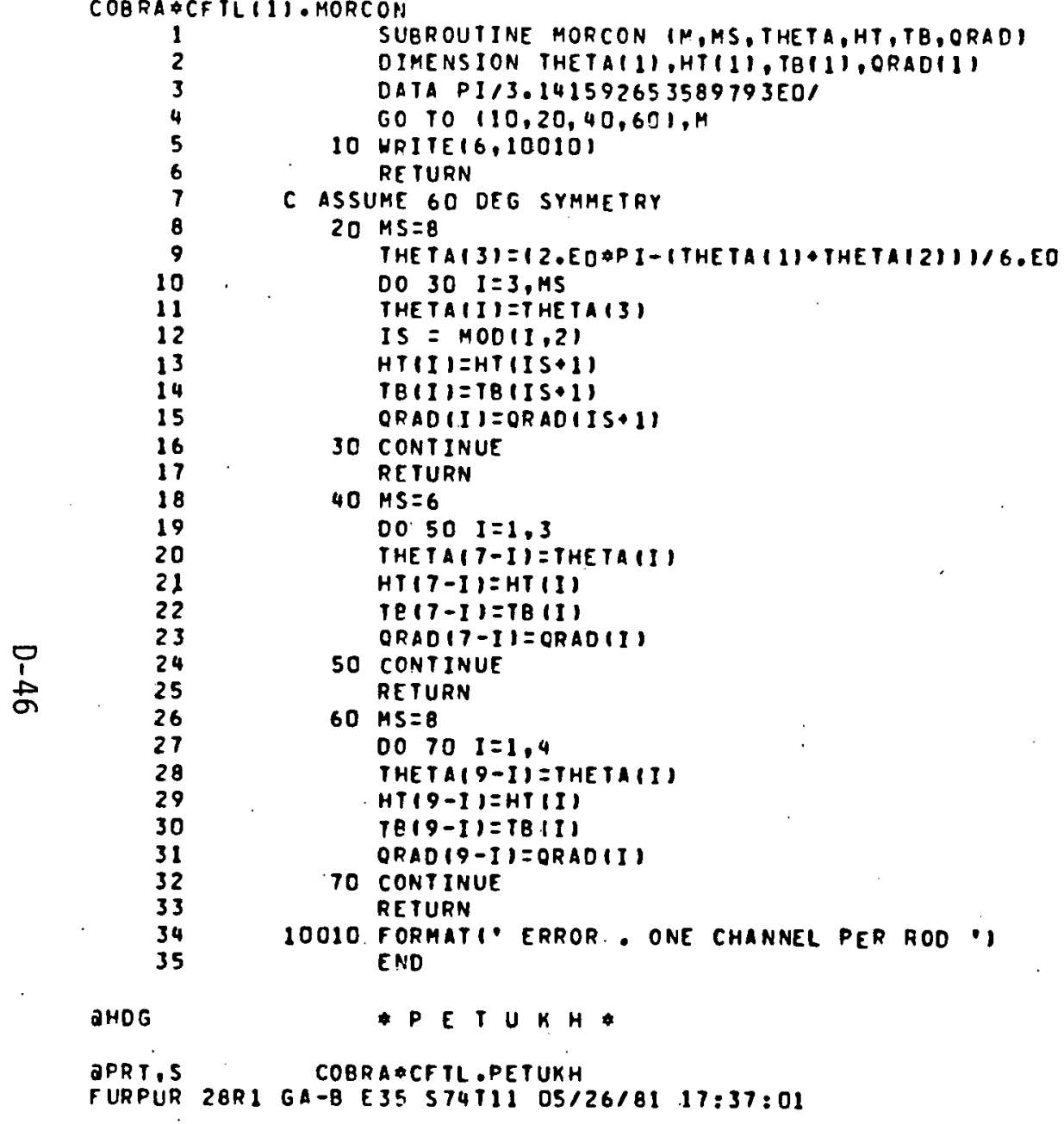


COBRAQCFIL (1), PETUKH

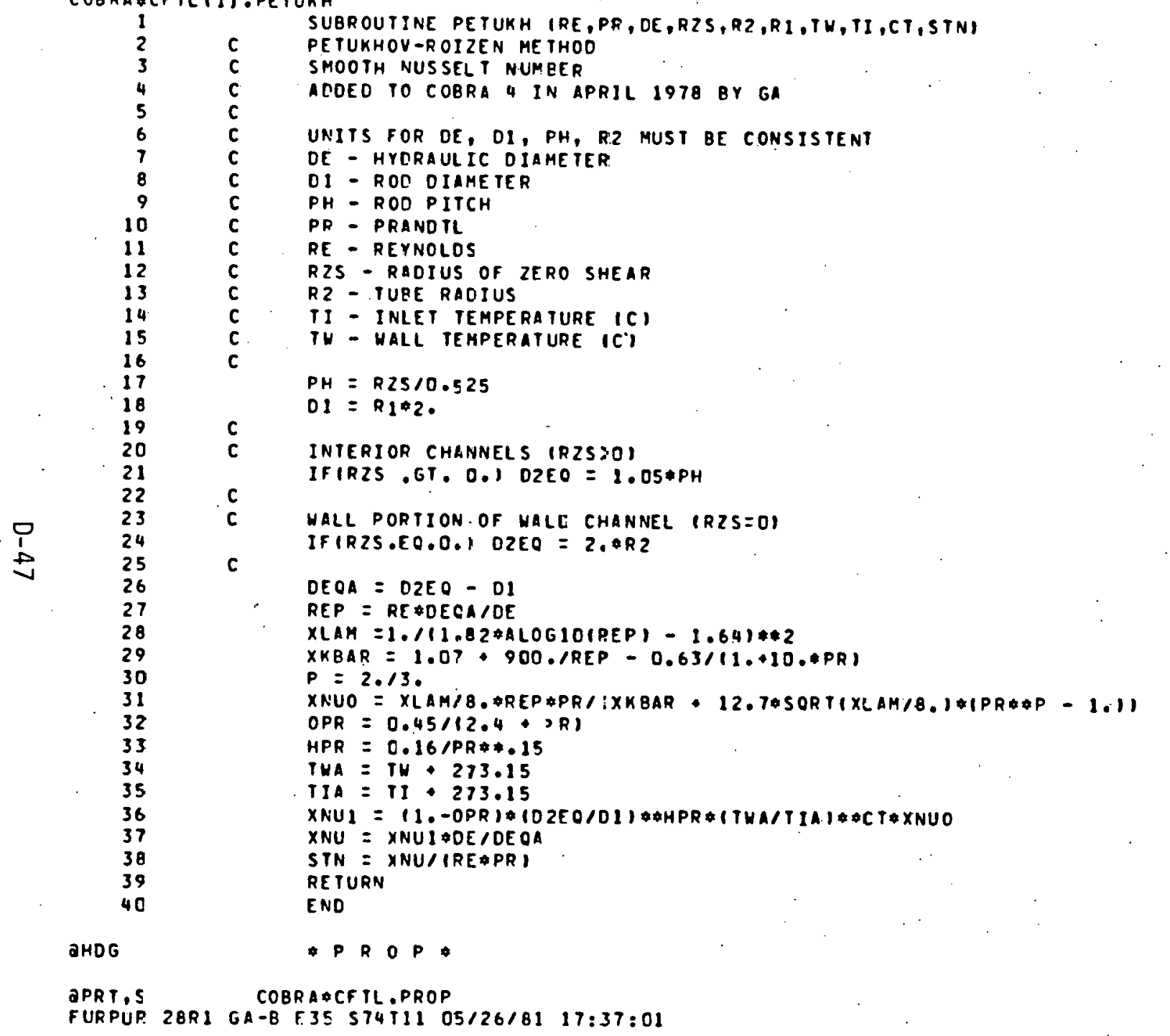

FURPUP 28R1 GA-B F35 574T11 05/26/81 $17: 37: 01$

121000

121100

121200

121300

121400

121500

121600

121800

121900

122000

122100

122200

22300

122400

122500

122800

122900

123000

123000

123100

123200

123300

123400

123500

123600

123800

123900

24000

124100

124300

124400

124500

124600

124600

124700

124800
124900 
COBRA CFTLI1) .PROP

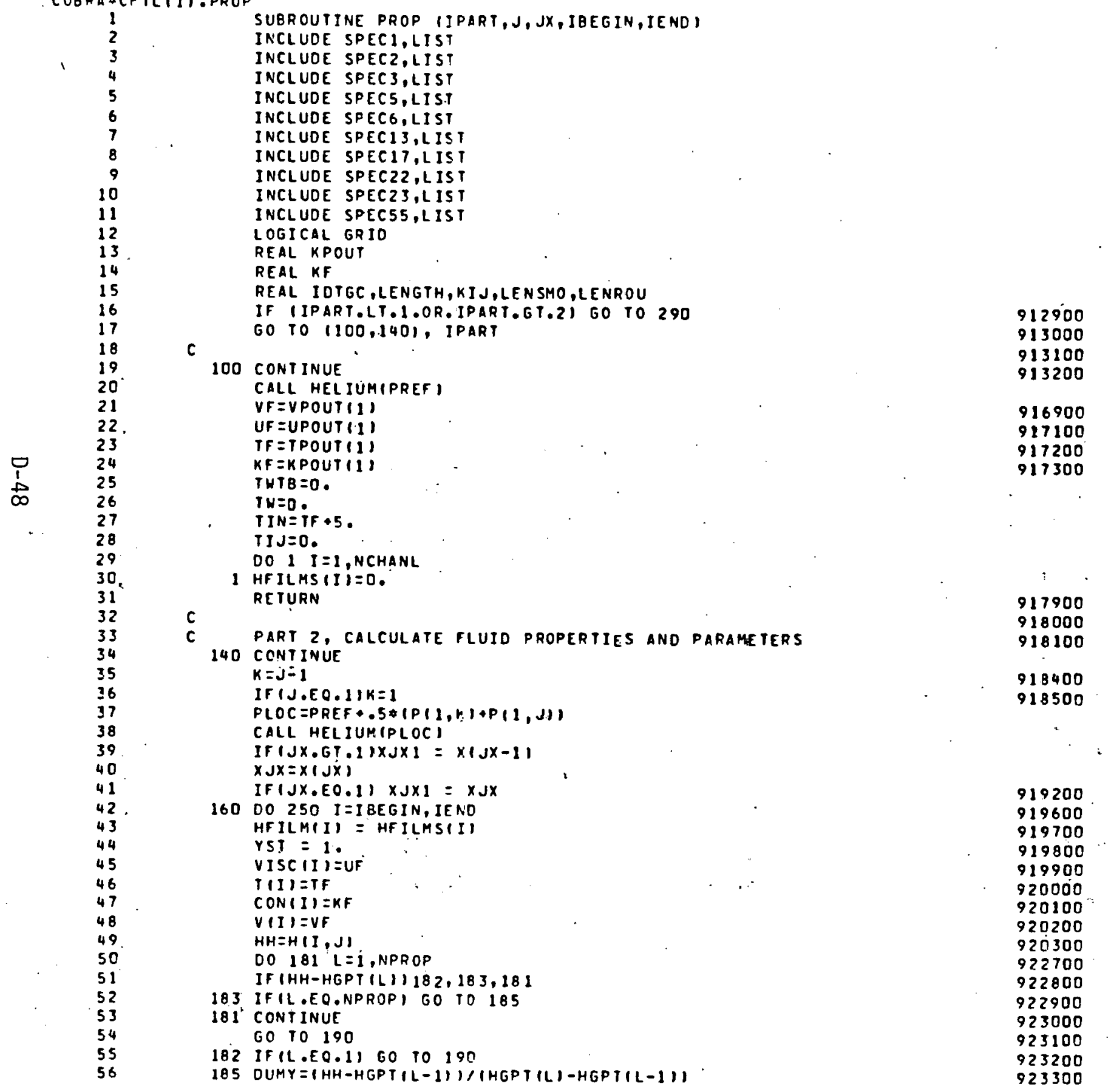


- P R O P.

VISCIII:UPOUTIL-L) DUAY I UPOUT ILI-UPOUT IL-III

VIII = VPOUT IL- I \& DUM Y I VO OUTILI-VPOUTIL- 11

III =TPOUTIL-1)・DUMY (TPOUTILI-TPOUTIL-II)

CON(I)=KPOUTIL-II+OUMY \& KPOUTIL)-KPOUTIL-1)

CPIII=CPPOUTIL-IIDOUMY \& CPPOUTILI-CPPOUTIL-III GO 10200

190 WRITE 113,3301

IERROR $=10$

RETURN

200 CONTINUE

RE = AHAXI(1, ABSIFII,J)/AII,J)ODHYDII,JI/VISCIII

RENOII,J) = RE

PR $=$ CPII I $A$ ISC I I ) /CONII)

IF INFH.GE.1) GO TO 66

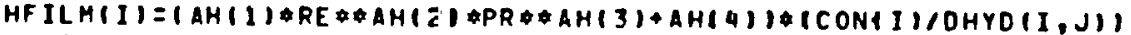

$L=N T Y P E I I I$

IFIL.GT.NFTIL = I

$F S P(I)=A A(L) \# R E * A B B(L)+C C(L)$

FLAM $=0$.

IF ILAMNF.GT.OI FLAM = ARLIL) \#RE \#BBLIL +CCLIL)

IFIFLAM.GT.FSPIIIIGO TO 210

GO TO 250

66 CONTINUE

923400

923500

923600

923700

923800

923800

923900

924000

924100

924200

924300

924400

924500

924600

924700

924700

927300

927400

CORRECT HEAT IRANSFER COEFFICIENT

IFIHFILMIII. EQ. O.I GO TO 69

IFISTNOII,J) .EO. 0.160 TO 69

c

XKC - THERMAL CONDUCTIVITY OF CLAOOING, KH/M-C

$X K C=0.01420 * 1.087 E-5 * \mathrm{TH}$

BIOT NUMBER CORRECTION

BIOT = HRIB AHFILMIIIIXKL

APPLY BIOT CORRECTION ONLY TO ROUGH SECTION

IF IXJX LE. LENSMO OOR, XJX GE. LENROUI BIOT=O.

HFILMII) = CONIII/OHYD(I,J)\#STNOII,J) WRE \#PR

HFILMII) = HFILMII $*(1,-B$ IOTC $*$ BIOT)

C SPACER EFFECTS ON HEAT TRANSFER COEF.F ICIENT

IFINGRID.LT.1) GO TO 34

DO 35 JG $=1$, NGRID

$X B S=2$ WR IDXL(JG) - SHQ0.5

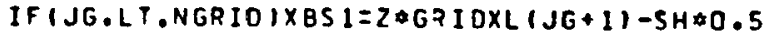

C DXH - NUMBER OF HYORAULIC DIAME TERS FROM BEGINNING OF SPACER $X D I F=X(J X)-X B S$

$S H H F=S H / 2$.

$J X H=X O I F / O H Y O I I, J)$

IFIJG.LT.NGRID)OXHI $=(X(J X)-X B 51) / O H Y O I I, J)$

IFIJG.EO.NGRIDIGO TO 36

IF IOXH.LT. $-1.1 \quad G 0$ TO 34

IF IOXH1.GT.-1.) GO TO 35

929800

GO TO 36

5 CONT

GO TO 34

36 CONTINUE

JGT = IGRIOIJG

$A D=S H H F / O H Y O I I, J I$

$C=A L O G 10(R E)$

IFIXJX.LT.LENSMO .OR. XJX.GT.LENROUI GO 1038
ROUGH BUNDLE

927800

927900

928200

928300

928600

928800

928900

929000

929100

929200

929300

929400

929600

930000

930100

930200

930300

930400 
IF $10 \times H . G T . A O I G O$ IO 32

AKRE $=.174$ * SORT (RE)

IFIRE. GT.2653.IAKRE $=3.3+72700 . / R E * \$ 1.2$

GO TO 39

931000

931100

38 IFIRE.GT. 3000 . IAD $=A D \$ 2$.

IF IOXH.GT.ADIGO TO 33

AKRE $=.174$ SORT(RE)

AKRE $1=(8.38 * 4550.1 R E * 0.8) * C O 11, J 6 T) * 0.4$

IF IAKRE I.LT.AKRE IAKPE = AKREI

39 CONTINUE

ANUSM $=$ AKRE $\triangle$ COO I I JSTIODO

931700

YST $=1 . \bullet A N U S M+10 X H \bullet 1,1 / 1 A D+1.1$

GO TO 34

$32 A M=1.855 E-3 \notin R E$

IFIRE.GT. 3000.1AM 1.04 .9E 4/RE \$1.2

$A K=4.42-1.05 * C$

AKONE $=-.344 \cdot 0.35 * C$

AKTHO $=-.1298 * C \# C+1.2466 * C-1.8478$

IF IRE.GT. 3000 , I AK = AMIN I I AKONE, AKTHOI

IF $1 A K \cdot L T .08851 A K=.885$

GO 1037

$33 A M=1.855 E-3 * R E$

IFIRE.GT. 3000 . IAM $=30.34 / R E \$ .253$

$A K=4.42-1.05 * C$

IF IRE. GT.3000. IAK $=.426+.113$ *C

IFIAK.LT..895 IAK $=.895$

$37 \quad A K=A K-2.25+C D 11, J G T I$

IFIAM.GT.4, IAM 4 .

$A M=A M \otimes C O I I, J G T I A \& 2$

$Y S T=A K /(O X H / R E / P R) * A M$

34 'IFIYST ELT.1. IYST $=1$.

HFILMIII = HFILMIIIOYST

C CALCULATE THTB FOR SUBCHANNEL I

931800

CALL CURVE (TIJ,HII,J),TPOUT, HGPT, NPROP, IERFOR, I)

NS $=0$

TH $=0$.

TWTB $=0$.

DO $68 \mathrm{~N}=1, \mathrm{NROD}$

DFLUX $=$ FLUX(N,J)

$0067 \mathrm{~L}=1, \mathrm{MSC}$

$I I=L R(N, L)$

IF III.NE.I) 60 TO 67

TH $=$ TH: IIJ DFLUX/HFILMIII

NS $=$ NS +1

60 TO 68

67 CONTINUE

G8 CONTINUE

IFINS.EO.O1 60 10 69

$T H=T H / N S$

$T W A=T H+273$.

$T E A=T I J+273$.

69 CONTINUE

IFILAMNF.EO.21GO 10242

c

FRICIION ANO STANTON NUMBERS

932000

932100

932200

932300

932400

932500

932.600

932700

932800

932900

932900

933000

933100

933200

933300

933400

933500

933600

933900

934000

934100

934200 
244. STNOII, JI $=$ STN

LAMARK II $)=0$

C XKC - THERMAL CONDUCIIIITY OF CLADOING, KHIM-C

BIOT NUMBER CORRECTION

939400

938500

933600

938700

938800

938900

939200

939500 
BIOI = HRIB*HFILMIII/XKC

C APPLY BIOT CORRECTION ONLY TO ROUGH SECTION

IFIXJX LLE. LENSMO OR XJX OE OLNROUI BIOT=O

HF ILMIII = CONIII DHYDII JIOSTNOII JI

HFILHIII = HFILMIIINIIO-BIOTCABIOTI Y ST

HFILMIII = HFILMII)*11.-BIOTC*BIOT)*YST

940300

$242 L=N T Y P E I I$

IFIL.GT.NFTIL=1

$F(A M=A A L(L) \otimes R E \otimes B B L I L)+C C L(L)$

IFILAMNF.EQ.2IGO TO 210

IFIFLAM.LT OFSPII 1$) G 0$ TO 244

210 LAMARK $(1)=1$

FSPIII =FLAM

C INCLUDE THTO IN CORPELATION FOR LAMINAR CASE

IFITHTB.GT.0.I FSPII) = FSP(I) THTB

FIND LAMINAR FLOH NUSSELT NO.

ROER Z (L)

IFIRO.LT .0.001)ROERZSILI

RAS =RR IL I/RO

IFIL.EO.1.OR.L.GT.3IGO TO 245

WALL OR CORNER CHANNELS

$A N U=4 \cdot 07+1.237 / R A S \$ 0.8027$

$60 T 0246$

245 CONTINUE

CENTRAL OR SUPPORT CHANNELS

ANU $=R A S /(1,+R A S) * 114.121+4.126 * A L O G 10.9523 / F A S-1.11$

$A N U=A N U \otimes D H Y O(I, J) / 2, / R O /(1,-R A S)$

246 CONTINUE

$X_{1}=X J X-X H$

IFIXI.LE.OIGO TO 247

C ENTRY LENGTH CORRECTION TO NUSSELT NO.

$D E I=2$ * \& RO -RR ILI)

GRI $=X 1 /$ IOEI $* R E \& P R I$

IFIGRI.GT.0.025IGO TO 248

$B=-0.1933+0.1217 / G R I * 0.1483$

60 To 249

$248 B=-1.338 E-3+2.772 E-5 / 6 R I * 1.763$

IF $1 B \cdot L T \cdot 0 \cdot 18=0$.

$-2 E-41 * B$

CORRECT FOR NON-ANNULAR WALL ANO CORNER CHANAELS

247 IFIL .EO.2.OR .L .EO. II ANU = ANU $\$ 0.967$

$A N J=A N U * Y S T$

SINO $(I, J)=A N U / R E / P R$

HFILHIII = ANUQCONIII/OHYOII,JI

TETAIII $=0$.

IFIL.EQ.1.0R.L.GT.3160 TO 243

WALL OR CORNER CHANNELS ONLY. COMPUTE EFFECTIVE SHROUO NUSSELT NO

IFIRAS.GT.0.1)GO TO 5

TETA2I $=-0.1033 * R A S * 0.9489$

60106

5 TETA 2I $=0.0142-0.07849 * R A S \$ 0.4828$

6 CONTINUE

IFIXI.LE . O.160 TO 10

IFIGRI.GT.0.01160 107

$Y T E 2=31 \cdot 105 * G R I$

GO 109

7 IFIGRI.GE.0.025IGO TO 
- P P OP:

285
286
287
288
289
290
291
292
293
294
295
296
297
298
299
300

aHOG

APRT $S$

CPRT, S
FURPUR 28R GABRA GACF TL,PROPS

$Y T=2=15.5994 * 6 R I * 0.8501$

8 YTEZ $=1 ., 10.9829+1.26 E-4 / G R I 0+2.2421$

IFIYTEZ.GT . I. IYTEZ $=1$.

GO TO 9

10 YTEZ $=1$.

9 TETA II) $=$ TE TA 2 I *YTE 2 *DEI/CONIII

243 HFILMSII) = HF ILMIII

250 CONTINUE

290 IEGROR $=11$

c

330 FORMAT I5X, OOU OF RANGE FOR PROPERTY TABLE, ENE

- F R O P S

FURPUR 28R1 GA-B E35 574TII 05/26/81 1.7:37:02

940600

941200

941300

941400

941500

942200 
COBRAFCFILU11.PROPS

1
2
3
4
5
6
7
6
9

QHDG

PPRT,S COBRAFCFTL,RADIAT

URPUR 28RI GA-B E35 S74T11 05/26/BI 17:37:02

INCLUOE SPECI3,LIST

IFIRZSILI.LE.0IGO TO 20

$$
\text { RETURN }
$$

20 CONTINUE

RETURN

END
SURROUTINE PROPSIRE,L,FRIC,SIN,I,JI

CALL BASICIRZS (L), RRIL), HRIB, RE, THTB,FRIC,SITN,I,J)

CALL IODTIHRIB,RE, RR(L),RZIL), THTB,FRIC,STN,I, JI

足 
COBRAOCFTLI), RADIAT

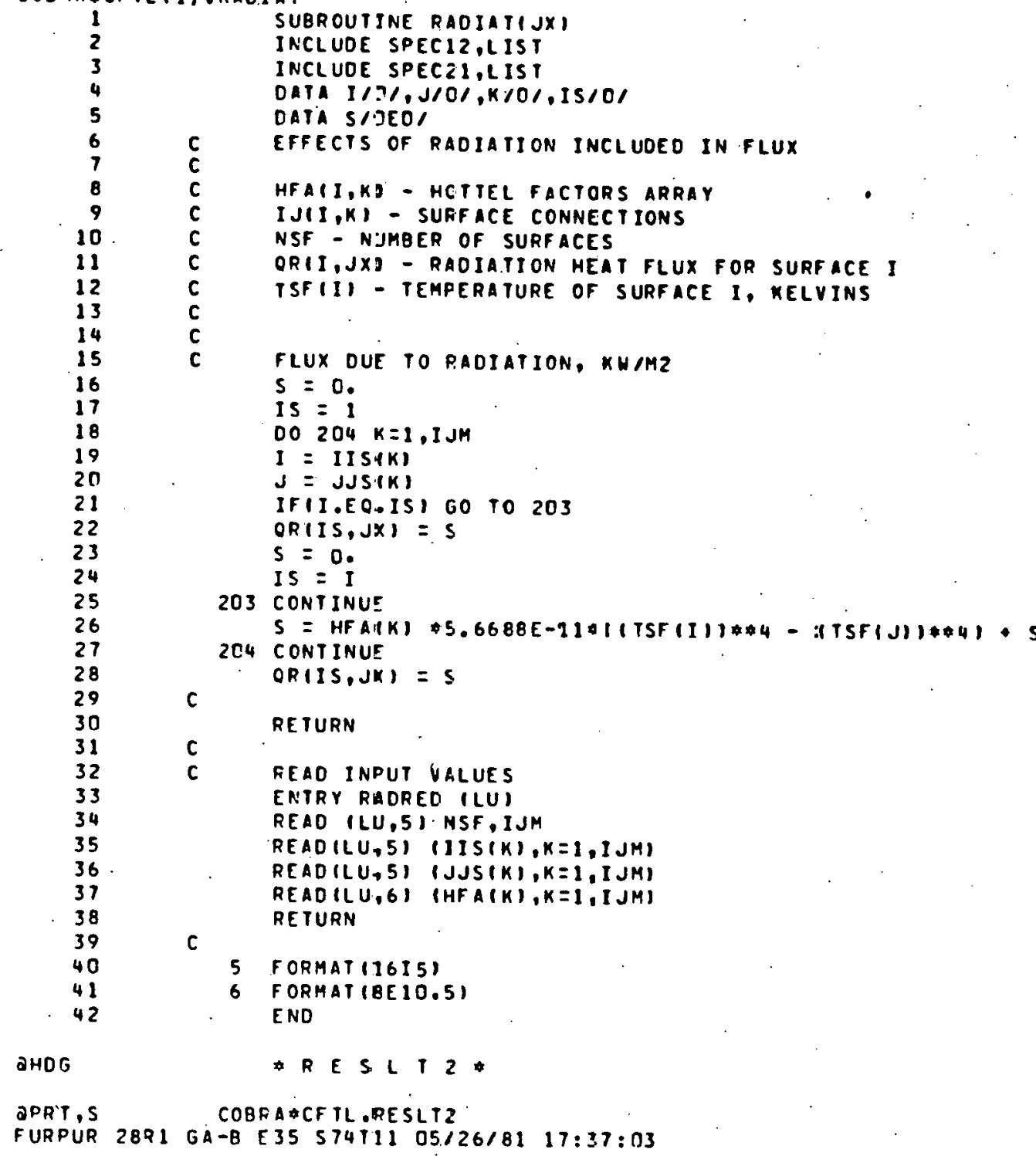

AHOG

DPRT, S COBFA \#CFTL DRESLT2

FURPUR 28R1 GA-B E 35 574T11 05/26/81 17:37:03

493800 495600 
COBPA $\$ C F T L 11$. RESL T 2

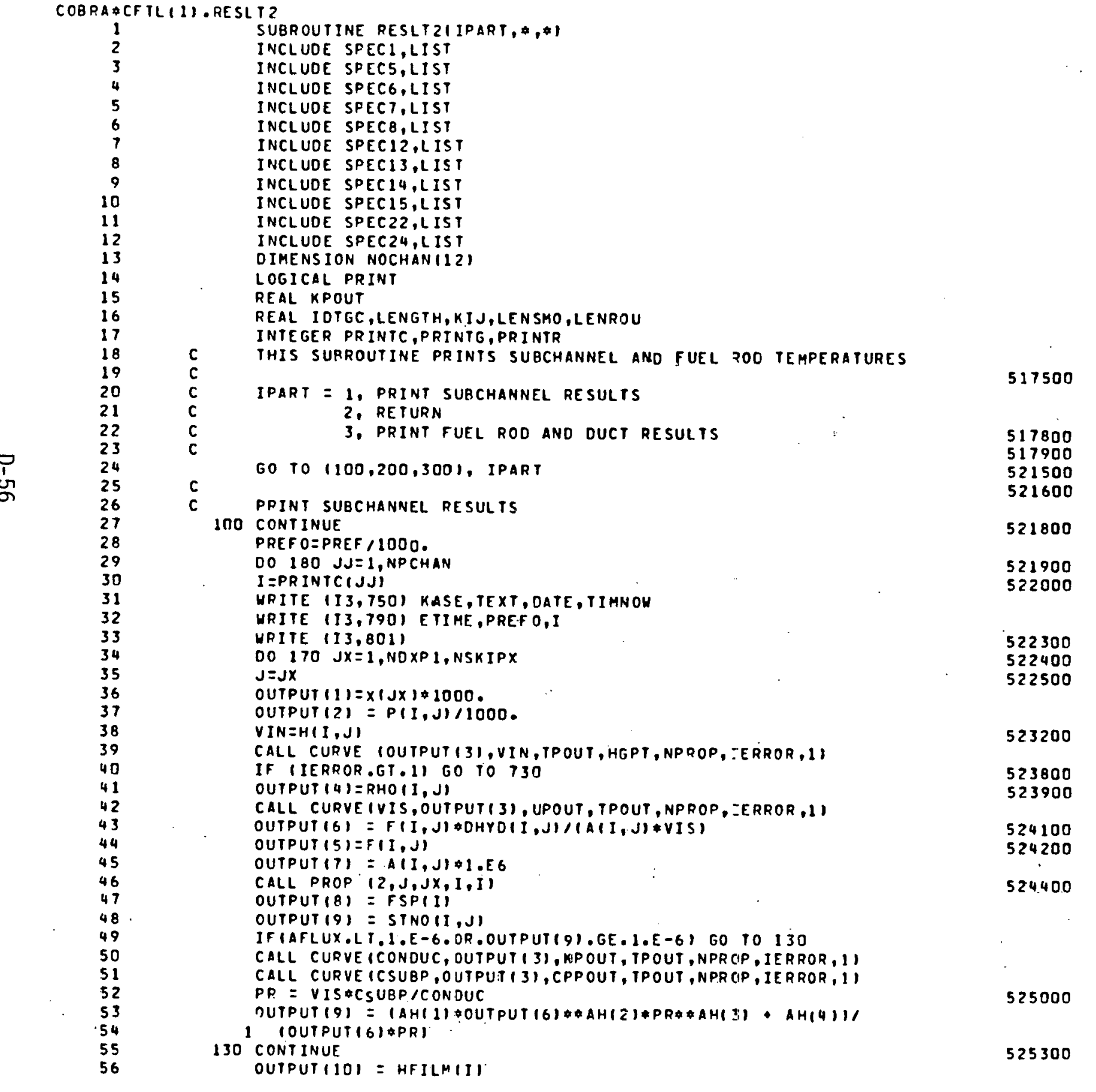


OUTPUTIIIIIEHPWII,J)

OUTPUT $(121=R H P(S I 1, J)$

IFIHPUII, JI.EQ0.0.1 AR =

OUTPUTI13I =AR

KRITEII3,8111 COUTPUIIII, II $=1,131$

170 CONTINUE

BO CONTINUE

RETURN 2

200 CONTINUE

C

RETURN 2

PRINT FUEL ROD RESULTS

3DO CONTINUE

DO 480 NN $=1, N P R O D$

$N=$ PRINTRINN

WRITE $(13,820)$ KASE, TEXT, DATE, TIHNOH, E.T IHE,

DO $470 J X=1$, NDXP1, NSKIPX

CALL CLOTMPIJX,NI

470 CONTINUE

c

480 CONTINUE

525600

525700

GA-MARB I

525900

526100

GA-MARB

531100

531200

531300

531400

531500

533600

533700

533800

534000

534100

534200

GA-MARB 1

534300

IF ( $(N F H-2)$ (NFH-4). NE D D)RETURN 2

$0027910 \mathrm{JX}=1$, NDXP 1

IF $(X(J X)$. LT. LENSMOV JXRB=JX

27910 CONTINUE

JXRB = JXRB + 1

$M A X C H N=0$

DO 27960 IC $=1$, NCHAN

NTYP = NTYPE(IC)

IF INTYP.GT . NF T INTYP=1

IF (R2INTYP) OEO. 0.160 TO 2795

MAXCHN = MAXCHN 11

NOCHANIMAXCHNI = IC

IF IMAXCHN G10 111 GO 1027920

GO TO $2795{ }^{\circ}$

27920 CONTINUE

PRINT 951, KASE, TEXT, DATE, TIMNOW, ETIME, (NOCHAN(II), I I I, MAXCHN) IF IMAXCHN.LT.12I PRINT 953

DO $27950 \quad J X=J \times R B, J \times R E$

$J=j x$

XOUMY $=x(1 \times 10100 \Omega$

DO $2794011=1, M A X C H N$

$I=$ NOCHANIIII

NTYP = NTYPE(I)

IFINTYP.GT . NFTINTYP=1

CALL PROP $12, J, J X, I, I)$

C DUCT HAS ONLY ONE ROD CONNECTED TO IT

$M=0$

DO $27931 N N=1, N R O D$

00279
$N=N N$

$N=N N$
$D O 27930 L=1$, MSC

IS $=L R(N N, L)$

IF IIS.GT.0I $M=M+1$

534600

534.700

534800

534900

535000

535100

535200

535300

535400

535500

535600

536300

536400

537400

537500

537600

537700

537800

531900

538000

538100

538200

538300

538400 


$$
\text { CALL ERROR }
$$

27932 CONTINUE

DFLUX $=F L U X(N, J)$

38600

IFIIFL.GT.O) DFLUX $=F\left(X\left(M_{,} J X\right)\right.$

CALL CURVE ITIJ,HII,JI,TPOUT,HGPT, NPROP, IERR JR, 11 539200

CALL CURVE (CPP,HII,J),CPPOUT,HGPI, NPROP, IER ZOR, 11

CALL CURVE IVSK, HII,JI,UPOUT, HGPT, NPROP, IERR OR, II

CALL DUCT IHRIB,RR (NTYP), RZINTYPI, HPLUS, GP PIME, OFLUX, TIJ,CPP, VSK, - THTB,BIOT,BIOTE,TO, I, AHSI

27940 CONTINUE

27950 CONTINUE

MAXCHN $=0$

27951 CONTINUE

IFIIC.LT.NCHANLI GO TO 27960

540000

540100

$\begin{array}{ll}\text { IFIMAXCHN.EO.0) GO TO } 27960 & 540400 \\ & 540500\end{array}$

$\begin{array}{lr}\text { GO TO } 27920 & 540600 \\ 27960 \text { CONTINUE } & 540700\end{array}$

RETURN 2

540700

$\begin{array}{ll}\text { ERROR EXIT } & 541000\end{array}$

$\begin{array}{ll}730 \text { CONTINUE } & 541100\end{array}$

GA-MARB I

750 FORMAT 117HICHANNEE RESULTS, $/ 5$ CASE, I5,5X17A9,9H DATE ,2A6,7HGA-MAR81 - TIME ,2A6/l

790 FORMAT 18 H TIME $=, F 8.5,9 H$ SECONOS, I3H PRESSURE =,F7.1,5H KPA, 541500 \25H JATA FOR CHAVNEL, 13.11

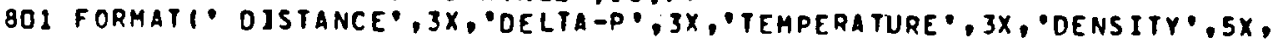

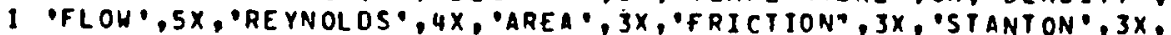

2 'FILM $H^{\circ}, 3 x_{1}, H+\cdots, 3 x_{0}: R(H+), 3 x_{0}, A R \cdot$.

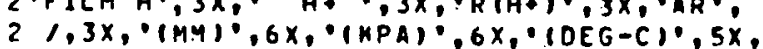

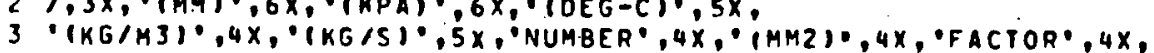

4 .

811 FORMAT I $2 x, F 6,1,3 x, F 8,3,5 x, F 6,1,7 x, F 5,3,4 x, F 8,6,4 X, F 7,0,1 X, F 7,2$,

$13 x, F 7,5,3 x, F 8,5,3 x, F 6,3,3 x, F 5,1,3 x, F 5,2,2 x, F 5,11$

820 FORMAT 15HICASE, I5,5X17A4,9H DATE, $246,7 \mathrm{H}$ TIME, $2 A 6 / / 8 \mathrm{H}$ TIME =GA-MAR8I - F8.5,9H SECONDS, $28 \mathrm{H}$ TEMPERATURE DATA FER ROD, I3,3X,10HIFUEL 542500 -TYPE, 3H - "CYLINOER',1HI)

951 FORMATI IOUCT TEMPERATURE RESULTS IDEG-CI,, $0^{\circ}$ CASE, I5,5X,16A4,A2 542700

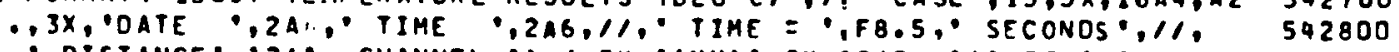
$\because$ distance, $121^{\circ}$ channel $: 1,1,3 x_{1} \cdot(m M), 2 x, 1213 x, \cdot 1 \cdot, 13, \cdot 1 \cdot, 2 \times 1,1$

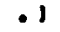

952. FORMATIF7.1,12F10.11

953 FORMAT $(1 X)$

543000

aHOG

* RESULT:

APRT,S. COBRAOCF TL.RESULT

FURPUR 28R1 GA-B E35 S74I11 05/26/81 17:37:03 
- re sul to

COBRA $*$ CF TLI1). RESULT

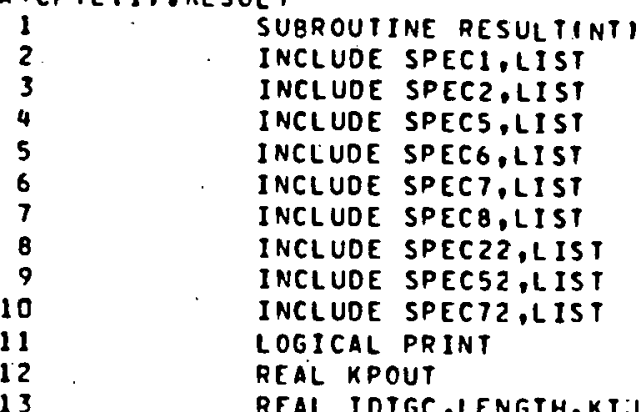

REAL IOTGC,LENGTH, KIJ,LENSMO,LENROU

INTEGER PRINTC, PRINTG, PFINTR

C THIS SUBROUTINE PERFORHS OUTPUT FUNCTION (PRINTINGI

FOR EACH TIHE STEP.

DATA. KT/OI

c

IF INT,EQ,1.OR,NT,EO,NDTPI) KT INSKIPT-1

$K T=K T+i$

IF (KT.LT.NSKIPT.AND.ETIME.LT.TTIME) GO TO 520

C

$K T=0$

PRINT RESULTS

IF IETIME.GT.O.I GO TO 160

c

COMPUTE MASS and ENERGY BaLANCE

FLOIN $=0$.

FLOOUT $=0$.

ENGIN $=0$.

ENGOUT $=0$.

NDXP1 $=N O X+1$

CO $100 \quad I=1$, NCHANL

IFINTYPEIII.GT.NFTIGO TO 100

FLOIN = FLOIN $+F(I, 1)$

FLOOUT =FLOOUT +FII, NOXPI:

ENGIN=ENGIN+F $\{1,1\} \$ H \mid I, 1)$

ENGOUT =ENGOUT +FII, NOXPII HII, NDXPII

100 CONTINUE

FLOERR =FLOOUT -FLOIN

ENGAOD $=0.0$

DO $104 \quad J=2$, NOXPI

$D X=X(7)-x(J-3)$

$00104 N=1$, NROD

DO $104 \quad L=1, M S C$

$1=L R(N, L)$

IFII.EO.DI GO TO 104

ENGADO = ENGADD PHI $(N, L) \& F L U X(N, J) \& P I \% D(N)$

-

CONTINUE

QDX

ENGERR =ENGOUT-ENGIN-ENGADO

ENGPCT $=0$.

IF. (ABS (ENGADD) . GT.1.E-3)ENGPCT = 100. O\#ENGERR/ENGADD

WRITE $(13,920)$ KASE, ITEXT, OATE, TIMNOW, FLOIN, ENGIN, FLOOUT, ENGADD,

FLOERR, ENGOUT, ENGERR, ENGPC

543600

546900

547400

547600

547700

547800

548100

548200

548400

548500 .

548600

548700

548800

548900

549000

549200

549300

549400

549500

549600

549700

550400

550500

550600

550700

550800

550900

551000

551100

551200

551300

551600 
C PREPARE CHANNEL EXIT SUMMARY

552400 $J=N D \times P \perp$

DO $110 \quad I=1$, NCHANL

$V I N=H(I, J)$

552500

CALL CURVE IOUTPUTIII,VIN,TPOUT, HGPT, NPROP, IERROR, 1 )

552600

OUTPUTI $21=F(I, J) / A N I I I / 1000$.

552800

110 CONTINUE

NOXPI $=$ NCX + 1

IF (IERROR.GT.1) 60 TO 330

554200

554300

554400

554500

COMPUTE BUNDLE AVERAGED RESULTS

554600

WRITE $(13,750)$ KASE, TEXT, DATE, TIMNOW

WRITE $(13,940)$

WRITE $(13,801)$

NOXP $1=N O X+1$

DO $150 \mathrm{JX}=1$, NOXP 1

$J=J X$

SAVE $1=0$.

SAVE $2=0$.

SAVE $2=0$.

SAVE $3=0$.

SAVE $4=0$.
SAVE $5=0$.

SAVE $5=0$.
SAVE $6=0$.

SAVE $6=0$.

DO $140 \quad 1=1$, NCHANL

IFINTYPE (I).GT.NFTIGO JO 140

SAVE I =SAVE $1+P(I, J)+A \mid 1, J 1$

SAVE 2 =SAVE $Z+H I I, J I Q F(1, J)$

SAVE $3=S A V E 3+F(I, J)$

SAVE $5=5 A V E 5+A(I, d)$

SAVE $6=5 A V E 6+P W(I)$

SAVE $4=5 A V E 4+R H O(I, J)+A \mid I, J)$

240 CONTINUE

OUTPUT $|11=\times| J \times 1 * 1000$.

OUTPUT $121=5 A V E 1 /$ SAVE $5 / 1000$.

OUTPUT I3I=SAVE $2 / S A V E 3$

CALL CURVE IOUTPUT 141 , OUTPUT (3), TPOUT, ,HGPT, APROP, IERROR, 11

554800

554900

555400

555600

555700

557000

557100
557200

557300

557400

557500

557600

557700

557800

557900

558000

558200

IF (IERROR.GT.1) GO TO 730

OUTPUT (5) = SAVE $4 /$ SAVES

OUTPUT $(6)=$ SAVE 3

CALL CURVE (VIS, OUTPUT (4), UPOUT, TPOUT, NPROP, IERROR, 1 )

OUTPUT(7) $=4$. OOUTPUT (6)/ (SAVE 6*VIS)

558400

558500

559100

559300

560100

OUTPUT $(8)$ = SAVE $3 / 5$ SAVE

OUTPUT $191=$ SAVE $51 . E 6$

WRITEII3,8III (OUTPUTIII, $I=1,9)$

150 CONTINUE

IF IIERROR,GT.11 GO TO 730

C

PRINT CHANNEL AND FOD RESULTS AS DEFINED EY DUTPUT OPTIONS

160 CONTINUE

c

PRINT SUBCHANNEL RESULTS

CALL RESLT2 $11,6181,6730$

181 CONTINUE

IF INOUT.EQ.0 .OR, NOUT,EO.1OI GO TO 510

IF INOUT.EO.2 OOR. NOUT.EOIIZI GO TO $34 \mathrm{D}$

IF INK.EO.OI GO TO $\$ 30$

5.61200

561300

561400

561500

562700

566200

566300

566400

566500 
$C$
$C$ PFINT CROSSFLOWS

566600

OO $210 \quad M=1$,NPGAP, 10

566800 $M M=M+9$

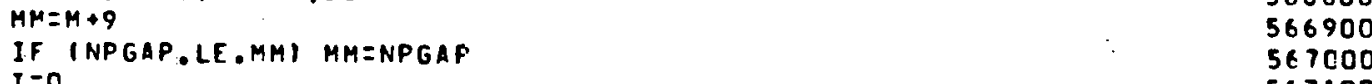

$I=0$

567100

DO 190 MKK $=M, M M$

567200

$I=1+1$

$1=1+1$

56.7200

567300
567400

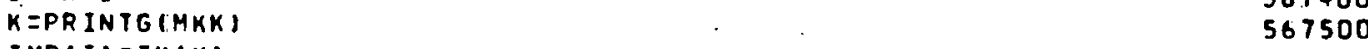

190 JMPII $=$ JKIK

567600

JMPII $=J K(K) \quad 567700$

WRITE II?,77O) KASE,TEXT, DATE, TIMNOW,ETIME, IIMP(I),JHP(I),

568000

DJ $210 \mathrm{~J}=1$, NOXP1,NSKIPX

$J=J x$

568000

$\begin{array}{ll}J=J X & 568100 \\ \text { If }(J X \cdot E Q .1) \text { GO } 10210 & 568.200\end{array}$

$x O M=x(J X-1) \neq 1000$

$X D U M Y=X(J X) * 1000$

DO $200 \quad I=M, M M$

II $=$ PRINTGII

568500

II $I=I-M+1$

200 OUTPUT (III) $=W(I I, J$

NDUM $=M M-M+1$

568700
568000

$\begin{array}{ll}210 \text { CONTINJE } & 569100\end{array}$

$\begin{array}{ll}330 \text { IF INOUT.EO.1 OOR. NOUTEEO.111 } 60 \text { TO } 510 & 582100\end{array}$

C

IF PNPROJ.LT.1I GO TO 510

582300

SI0 WRITE II.3.780, ITERAT $\quad 593100$

520 RETURN

STRN

730 IERROR $=1$

603500

$\begin{array}{ll}\text { RETURN } & 603600\end{array}$

C

750 FORMAT I17HICHANNEL RESIJL IS, 15 H CASE, I5,5X17A4,9H

760 FCRMAT $(1 X, F 6,1,2 \mathrm{H}-, \mathrm{F} 6.1,10 \mathrm{~F} 11.5)$

GA-MAR8 1

770 FCRMAT 168 HOI VERSION CZOSSFLO BETHEEN ADJACENT CHANNELS, $N(1, J), 604400$ - (KG/SEC-M). I/SH CASE,I5,5X,17A4,9H DATE, 2A6,7H TIME, 2A6//GA-MARB $7 H$ TIME $=, F 9,5,8 H$ SECONDS $/$,

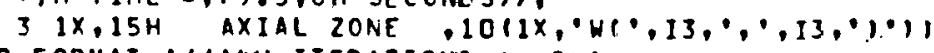

780 FORMAT I//14H ITERATIONS $=$. I4I

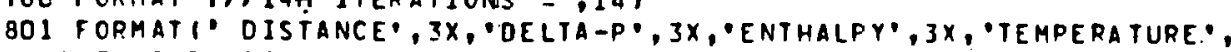

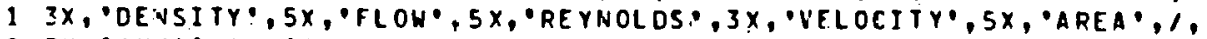

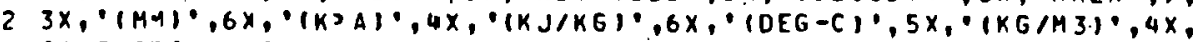

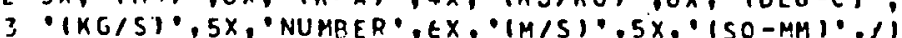

811 FORMAT I $2 X, F 6,1,3 x, F 8,3,3 x, F 7,2,7 x, F 5,1,7 x, F 5,3,5 x, F 6,4,5 X, F 6,0$, $6 x, F 6,3,6 x, F 5,11$

920 FORMATIIHI, CHANNEL EXIT SUMMAPY RESULTS ,,$\cdot$ CASE $, 15,5 X, 1744$,

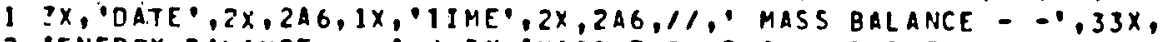

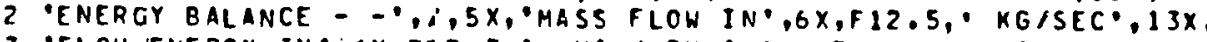

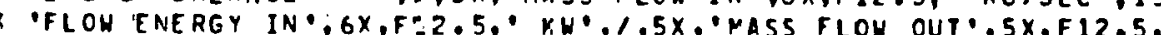

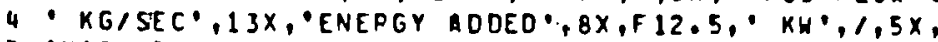

5 'MASS FLOW ERROR", 3X,E12.5, "KG/SEC, $13 x$, "FLOW ENERGY OUT', $5 x$,

604800 


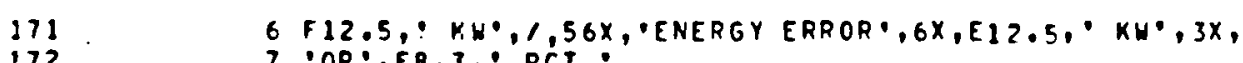

$1727{ }^{\circ} O R^{\circ}, F 8.3,0^{\circ} \mathrm{PCT}, \circ$

173

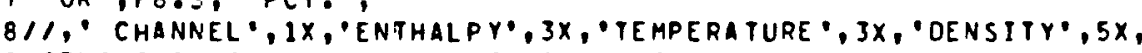

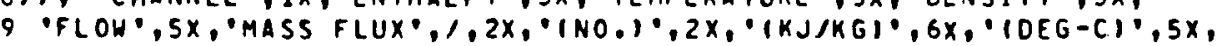

$\left.1 \cdot(K G / M 3)^{\circ}, 4 x_{0} \cdot(K G / 5) \cdot, 4 x_{1} \cdot(G / 5-M M 2),, 1\right)$

930 FORMATII5, 4X,F 7.2,7X,F5,1,7X,F5,3,4X,F7,5,5X,F6.4

940. FORMAT IIX,24H BUNDLE AVERAGED RESULTS\%)

END

611000

- RODCON

612500

aHOG

COBRA $\$ C F T L . P O O C O N$

APRT,S COBRA \$CF TLPOOOCON 
COBRA QCFTLIII.RODCON

SUEROUTINE ROOCONINROD,JX,TBLK, HTC, OFX, OII

INCLUDE SPECG, LIST

INCLUDE SPEC12,LIST

INCLUDE SPECZ1.LIST

INCLUDE SPEC16,LIST

DIMENSION ORAD $(24)$

DIMENSION TBLKI11, HTCII:

$C$ DIMENSIONS OF $T, Q$, RHS ARE NOIM=(NR-1) $\# M S+1$

C DIMENSIONS OF HT, PB, THETA ARE MS =BANDHIDTHEMAX NUMBER OF CHANNELS

C LEADING DIMENSION OF A MATRIX $=M S+1$

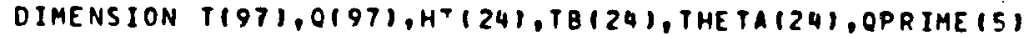

OIMENSION A $(25,97)$, RHS 197$)$

C LR(N,K) - THE NUMBER OF THE KTH SUBCHANNEL CONNECTEO TO ROD N.

C PHIIN,KI - THE FRACIION OF THE KTH SUBCHANNEL CONNECISD TO ROD N.

TBLKII,J) - BULK TEMPERATURE OF SUBCHANNEL I AT AXIAL LOCATION J

DFLXIN,J) - HEAT FLUX FOR ROD N AT AXIAL LOCATION J

HSIN,I,J) - HEAT TRANSFER COEFFICIENT FOR ROD N, SUBCHANNEL CONNEC

AXIAL LOCATION J

C MUST USE CCNSISTANT UNITSJJJJJ

DATA THOPI/6.283185307EJ/

C DEFINE NR AND ITHETA NOW. I VPUT THEM LATER

$N R=3$

ITHE TA $=1$

$M T=0$
$D O \quad 280 \quad N=1, N R O D$

DFLX OOFX RADIALIN

DO 10 II $=1$, MSC

$I=L R(N, I I)$

IFII.EO.0IGOTO20

$M=M+1$

THETAIIII $=$ TWOP I*PHIIN,III

TBIIII $=$ TBLKIII

HTIII) $=$ HICIII

C MUST FUDGE PAOIANT HEAT FLUX AS LINEAR HEAT SINKISOURCEI IN CLAD

c ORADIIII =ORIII +MT, JXIARF (NR) THETAIIII

10 CONTINUE

20 CONTINUE

DO $30 \quad I=1$, NCQNR

IFIN.EQ.ICONAIII), OOTOSC

30 CONTINUE

C DO NOT CONSIDER CONDUCTION ON THIS ROD DO $40 \mathrm{~J}=1, \mathrm{M}$

$F L X(M T+J, J X)=D F L X$

$T S F(M T+J)=T B(J)+273$.

40 CONTINUE

$M T=M T \cdot M$

GOTOZOO

50 CONTINUE

C CONROD NEEOS LINEAR HEAT FLUX FOR EACH INTERNAL RADIAL PING

$0060 \quad 1=1$, NR 
60 OPRIME III =DFLX $\$$ TWOPI $\$ R F I N R)$ OPFRACIII

$$
M S=M
$$

IFIM.LT.5) CALL MORCON (M,MS,THETA,HT, TB,OZAD)

MSO $=$ MS

GO TO $170,80,1001$, ITHETA

70 CONTINUE

$M S P 1=M S+1$

GO TO 120

BO CONTINUE

$M S=2$ a MSE

$M S P 1=M S+1$

$M S M 1=M S-1$

DO $901=1, M S H 1,2$

MSPIMI $=$ MSPI - I

MSOMI2 $=$ MSO $-I / 2$

MSMI = MS - I

THE TAIMSPIMI I IHETAIMSOMI $21 / 2.0$
THETAIMSMII THETAIMSOMI $21 / 2.0$

GA-MARB 1

GA-MARB I

GA-MARBI

GA-MARB I

GA-MARBI

GA-MARB 1

GA-MARB I

HT(MSMI) $=$ HIIMSOMI2)

TB(MSPIMI) $=T B$ IMSOM I2।

AR8I

ORAOIMSPIMIIIIORADIMSOMI 21

GA-MARB 1

ORAD (MSMI) =ORAD (MSOMI 21

GA $-M A R B 1$
GA-MARB I

90 CONTINUE

GO TO 120

100 CONTINUE

$M S=3+14 S O$

$M S P 1=M S+1$

$M S M 1=M S-1$

DO $110 \quad I=1, M S M 2,3$

MSPIMI $=$ MSPI - I

MSOI $=$ MSO- $-1 / 3$

MSOI $3=$ MSO - I
MSMI $=$ MS - I

MSMIMI $=$ MSMI - I

THE TA (MSPIMI) = THETAIMSOI31/3.0

THE TA (MSMI) = THETA (MSOI $31 / 3.0$

THETAIMSMIMII = THETAIMSOI $31 / 3.0$

HTIMSPIMII $=$ HI IMSOI 3 I

HTIMSMII $=$ HTIMSOI 3 I

HTIMSMIMI) $=$ HT (MSOI 3 I

TE(MSPIMI) $=$ TB(MSOI3)

TB(MSMI) $=$ TB(MSOI3)

TE(MSMIMI) $=$ TB (HSOI 3$)$

OFAD (MSPIMI) ORADIMSOI

ORAD (MSMI) =QRADIMSOI3)

OFADIMSMIMII =ORAOIMSOI3I

110 CONTINUE

c

120 CONIINUE

NOIM $=(N P-114 M S+1$

DO $130 \quad I=1$, NOIM

$130 T(1)=0.00$

c

CALCULATIONS

GA-MARB ?

GA-MARB I

GA -MARB I

GA-MARB I

GA-MARB I

GA-MARBI

GA-MARB I

GA-MARB I

GATMARBI

GA-MARB I

GA-MARBI

GA-MARB 1

GA-MARB 1

GA-MARBI

GA-MARB I

GA-MARBI

CALL CONRODIA,T,RHS, O, OPRIME, ORAD, IB, HT, THETA, OT, MSPI, MS, NOIM, NRI 


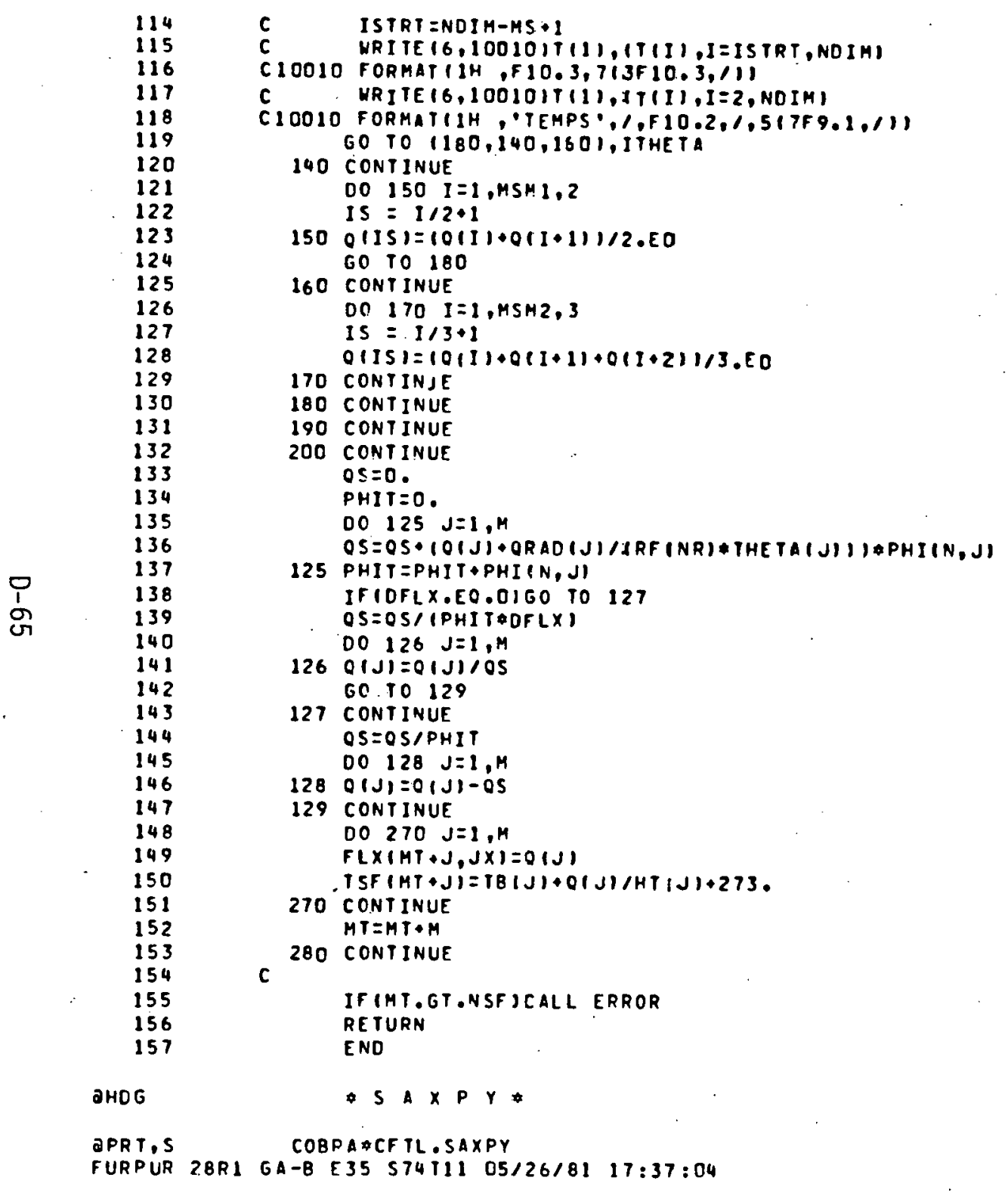

GA-MARBI

GA-MARB I

GA-MARE 1

GA-MARB I 
COBRA CFTL11). SAXPY

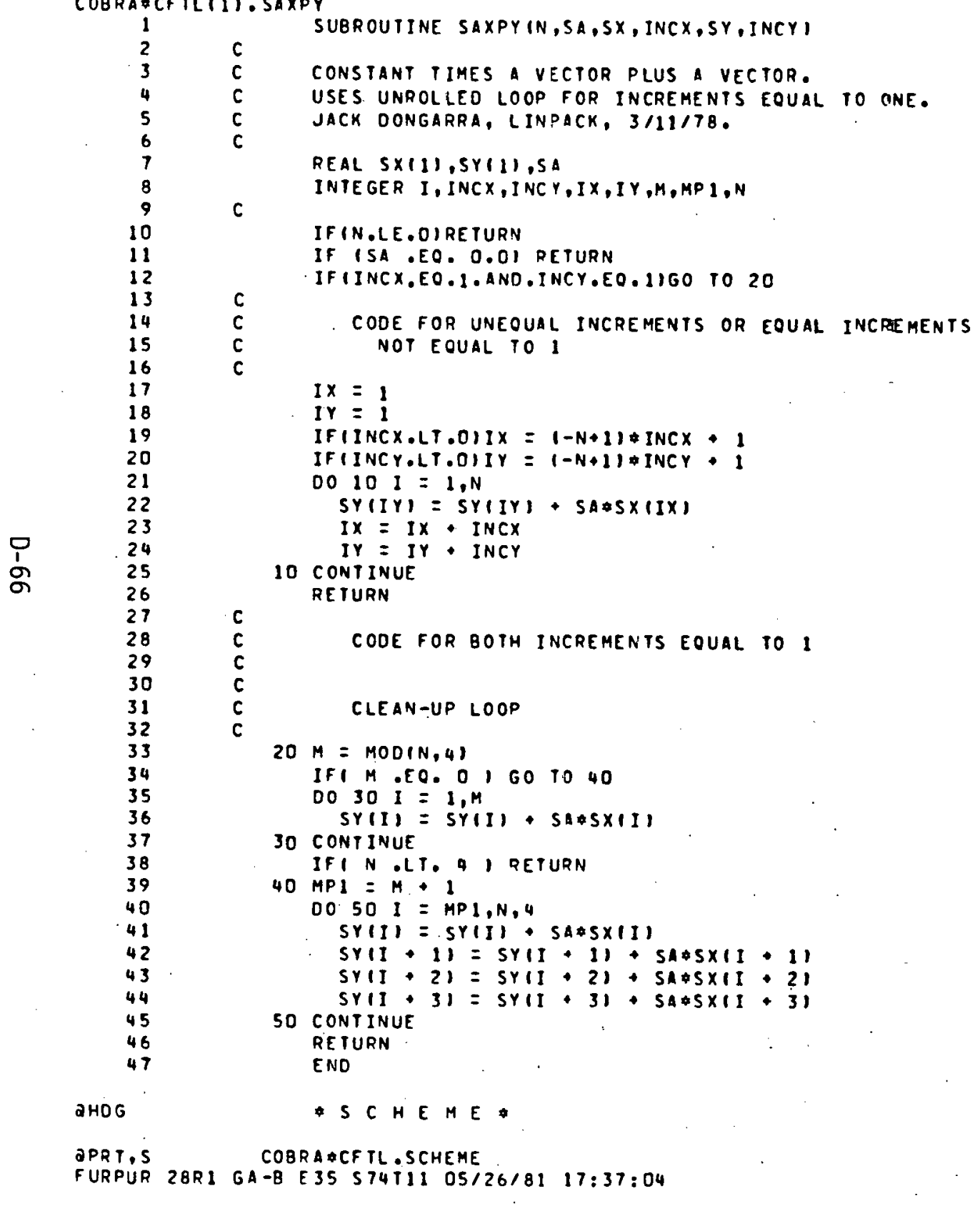


COBRA \#CF IL 111 . SCHEME

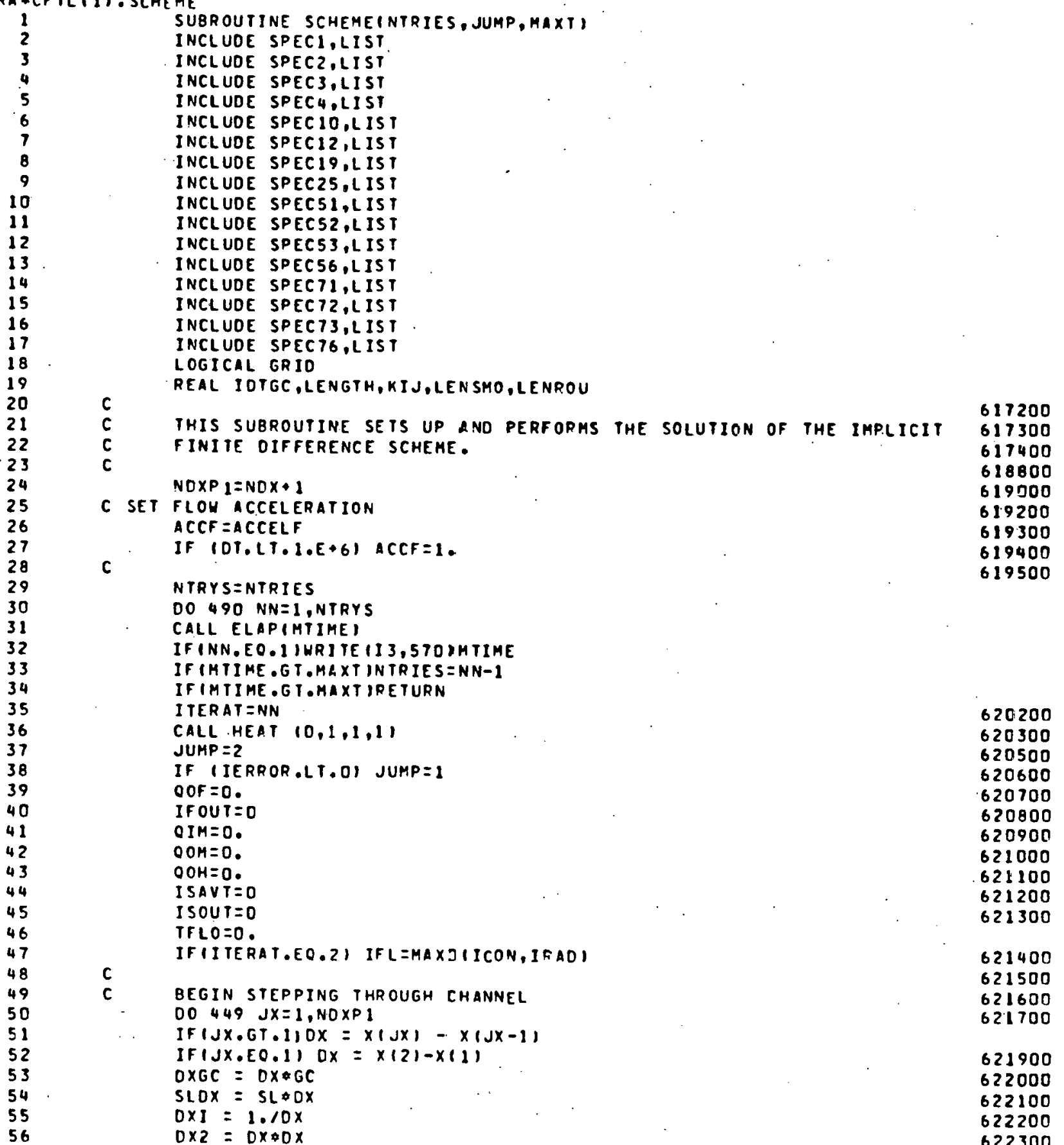

617200

617400

618800

619200

619300

619400

(DT.

NTRYS $=$ NTRIES

IFINN.EO.1)HRITE 113,570IHTIME

IRE.G T.MAXT INTRIES =NN-1

TTERAT = NN

$10,1,1,1$

IF IIERROR.LT.OI JUMP =1

OF $=0$.

620300

620500

620700

-1
0

621000

21100

21300

621400

21900

22100

622300 
623500 IF IIERROR.GT.1I GO TO 430 IF IJ.GT.1IGO TO 110

623600 $\begin{array}{ll}\text { SET CONDITIONS AT START OF CHANNEL } & 623700 \\ \text { DO } 100 \text { I I, NCHANL } & 623800\end{array}$ TFLO $=T F L O+F(1,1)$

100 OPRIMIII $=0$.

GRID =.FALSE.

624000

624100

624200

624200

CALL PROP $12, J, J X, 1, N C H A N L)$

$\begin{array}{ll}\text { IF IIERROR.GT.11 GO TO } 430 & 624400\end{array}$

624300

RHOII, $10=1$ (

UBII, I I $=F(I, 1) * V(I I / A \mid I, 1)$

CONTINUE

IF IIERROR.GT.1) GO TO 430

CALL FORCE $(J, J X, J)$

GO $10 \quad 440$

C CALCULATE PARAMETERS TO BE SAVED FROM PREVIOUS SPACE

624600

624700

624900

625000

625100

625200

625300

$F(1, J)=F(1, J F)$

$R H O(I, J)=R H O(I, J F)$

$H(I, J)=H(I, J F)$

$U B(I, J)=U B(I, J F)$

VPAIII $=V I I I / A(I, J M I)$

OPK $(I)=0$.

IFINN.GT.1.OR.OT.LT.1.E+6) GO TO 120

625400

$\begin{array}{ll}0 & 625700\end{array}$

TFLOJ:TFLON+FiI JI

120 CONTINUE

CALL HEAT $(0, J, 2, J \times)$

IFINSCBC.EQ.4) CALL PROP(2,JI,JX,1, NCHANL,

625700

625800

626000

626100

626200

626300

626400

IF IIERPOR GT II GO TO 430

CALL DIFFER $(1, J, J X)$

IF IIERROR.GT.I) GO TO 430

$c$

SIMULTANEOUS SOLUTION FOR ENTHALPY

$A C C E L H=1.5$

DO $130 \quad I=1$, NCHANL

IF (NN,EO.1.ANO.OT,GE.1.E+6) F(I,J)=F(I,J)\#TFLOITFLOJ

626400

626500

626600

626700

626800

626900

627000

627100

627200

627300

627300

627400

If $(N K \cdot L E \cdot D) \quad G 0.70 \quad 150$

DO $140 K=1, N K$

627500

627600

627700 Jis $=1 k(k)$

FLOW (II) =FLOW(II)+H(K;J)

140 LLOW (JJ) $=$ FLOH $(J J)-h(K, J)$

150 CONTINUE

HERR OR $=1, E-6$

DO $160 \quad I=1, N C H A N L$

627900

628000

628100

$M=1 \cdot 11-11$ ONAAH

628200

628300 
$A F=(R H O O L O I I, J) / O A P P I I, J) / O T$

FJUMY $=F 11, J M 1100 \times 1$

OUMY $=$ DHDXIII

$A A H(M)=A R \cdot F D U M Y-F L O N I 1)$

628600

DHDX(I) =DUMY $+A R * H O L O(I, J 1$

IF (NK.EO.O.AND.NHK.EO.O) GO TO 320

DO $200 I=1$, NCHANL

$M=(1-1)$ ONAAH

$N=(I-1)$ NA AHP 1

IEND $=I L O C(N+N A A H)$

$M P 1=M+1$

IF IIEND.LE.OI GO 10180

$00170 L=1$, IENO

$K=I L O C(N \bullet L)$

$A B W=A B S I W(K, J)$

DUMY = AMINO I, IABSII-ISTAR (K))

AAH (MP I I = A AH (MP I) 11 -OUMY) $\triangle B W \bullet W P P(K)$

170 AAH $(M P 1+L)=-D U M Y A B H-W P P(K)$

180 IF (NWK.EQ.0) GO TO 200

IENDS = ILOC (N+NAAHPI)

IF IIENDS.LE.OI 60 TO 200

$N P P=N \cdot I E N D$

$M P P=M P 1+I E N D$

DO $190 \mathrm{~L}=1$, IENDS

$M P P L=M P P+L$

$K=I L O C(N P P+L)$

INOEX $X$ I-MIND(1, I ABSII-IKW(K)) +2*(1-MINOI, IAESII-JKW(K) )

AAH (MP I) = AAH (MPI) UUCPIINDEX,K) \&HIOTH(K)

AAH (MPPL) =-UHALL (INOEX, K) \#HIDTH(K)

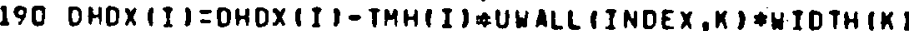

200 CONTINUE

DO $210 \quad I=1$, NCHANL

$M=N A A H * \mid I-11$

DUMY $=A C C E L H / A A H(M+1)$

DHOXII $)=$ OHOXII I DUMY

DO $210 L=1, N A A H$

$M P L=M+L$

AAH IMPL) $=A A H(M P L)$ OUMY

210 CONTINUE

IF (NHK.EO.O) GO TO 230

I $A A K=N C H A N L$ NAAH

IAAK $=$ NCHANL $\#$ NAAH
DO $220 \mathrm{~K}=1$, NHK

$00220 K=1$, NHK
$M=1 A A K+(K-1) \neq 3$

$M=I A A K+(K-1)$ *

$I I=I K W(K)$

DUMY $=1$ RHOL CP $(K)<O T+U H A L L(1, K)+U M A L L 12, K) 11 / A C C E L H$

628700

628800

628900

629000

629100

629200

629300

629400

629500

629600

629700

629800

62.9900

$6 \geq 0000$

630100

630200

63.0300

630400

630500

6.30600

630700

630800

630900

631000

631100

631200

631300

631400

631500

631600

631.700

631800

631900

632000

632100

632200

632300

632400

632500

632600

632700

632900

633000
633100

$A A H(M+1)=-U W C P(1, K) / O U M Y$

633100
633200

$\triangle A H(M+2)=-U W C P(2, K) / O U M Y$

$A A H(M+3)=\{T M H(I I)$ UHALL $(1, K)+T H H(J J)+U N A L L(2, K)+R H O L C P(K) / O T$ TWOLO - IK,JIIIOUMY

220 CONTINUE

CONTINUE

633300

633400

633500

DTERATIVE SOLUTION

DO 310 IHH $=1$, I TRY

633600

633700

EFR $=0$.

633800

DO $280 \quad I=1, N C H A N L$

633900

634100 
$C=D H D X(I)-A C C E L H+H(I, J)$

IENOEILOC(N\&NAAH)
IF (IENO.LE.OI GO TO 250

IF IIEND.LE.OI

$D O 240 \quad L=1$, IENO
$K=I L O C(N+L)$

$K I=I K(K)+J K(K)-I$

$240 C=C-A A H I M+(L) * H \mid K I, J)$

250 CONTINUE

IENOS ILOC (N+NAAHP I)
IF IIENDS.LE.O) GO TO 270

$M P P=M+I E N D$

$N P P=N+I E N D$

DO $263 \quad L=1$, IENDS

$M P P L=M P P+L$

$K=I L O C$ (NPP $\&$ )

$260 C=C-A A H(M P P L) * T H A L L(K, J)$

270 CONTINUE

ERR = AMAXI(ERR, ABSIC/H(I,J)

$280 H(I, J)=H(I, J)+C$

IF (NWK.LE.0) GO TO 300

DO $290 K=1$, NHK

$+(k-1) \neq 3$

$I I=I K N(K)$

\section{$J J=J K H(K)$}

$C=A A H(M+3)-A C C E L H * T H A L L(K, J)-A A H(M+1) \& H(I I, J)-A A H(M+21 \& H(J J, J)$

THALL $(K, J)=T$ TWLL $(K, J)+C$

290 CONTINUE
300 CONTINUE

$O O H=A M A X\{(O O H, E R R)$

IF IERR.LT.HERRORI GO TO 340

310 CONTINUE

GO TO 340

320 DO $330 \quad 1=1$, NCHANL

$M=1+1 I-11+\mathrm{NAAH}$

$330 \mathrm{H}(\mathrm{I}, J)=D$ HOXIII/AAH(H)

c

340 CONTINUE

CALL PROP $12, J, J X, 1, N C H A N L$

IF IIERROR.GT.1) GO 10430

DO $2 I=1$, NCHANL

RHOII,JIII:IVIII

$U B(I, J)=F(I, J)+V(I)(A \mid I, J)$

2 CONTINUE

IF IIERROR.GT.11 GO TO 430

CALL FORCE IJ,JX,JMII

$63420 \mathrm{C}$

634300

634400

634500

634600

634700

634800

634900

635000

635100

635200

635300

635400

635500

635600

635700

635800

635900

636000

636100

636200

636300

636400

636500

636600

636700
636800

636900

637000

637100

637200

637300

637400

637500

637600

637700

637800

637900

638000

638200

IF IFTM TEROR.GT II GO TO 430

CALL DIFFER $(3, J, J X)$

IF (IERROR.GT.1) GO TO 430

CALCULATE THE DIVERSION CROSSFLOH AT $X_{0}$

638400

638700

638700

638800

638900

639000
639100

639200

IF (NK.GT.OI CALL DIVERT IJ,JX)

IF IIERROR.GT.11 GO TO 430

C CALCULATE THE FLOW I $X$ AND CHECK FOR CONVERGENCE.

639500

DO $350 \quad 1=1$, NCHANL

639600

639800

350 DFDX(I) $=0$. 
$\begin{array}{ll}00360 K=1, N K & 630000\end{array}$

$I I=I K(K)$

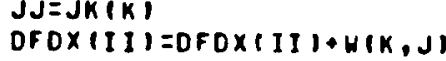

640100

640200

360 OROX(J) =OFOX(JJ)-H(K,J)

640400

640500

DO $380 \quad I=1, N C H A N L$

FSAVE $=F(1, J)$

640600

640600

OUMY =APP II JI:1RHO II, JI-RHOOLDII JIIIOT

640800

USERS MAY WISH TO TRY OTHER COMBINATIONS OF CONSTANTS. $\quad 640900$

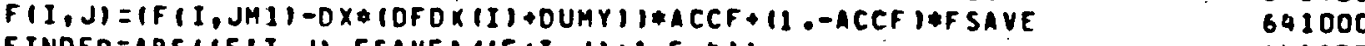

FINDER = ABS (IFII,J)-FSAVEI/FII,J)+1,E-4)

641100

QUAN = AMAXI IOUAN, FINDER I

380 CONTINUE

IF IOUAN,GT.FERR OR:OR.IERROR .LT.OI JUMP =1

IF INK.GT.OI CALL DIFFER $14, \mathrm{~J}, \mathrm{JX}$ )

IF IIERROR.GT,1) GO TO 430

IF INK.EO.01 GO TO 400

DO $390 \quad K=1, N K$

$I I=I K|K|$

$J J=J K(K)$

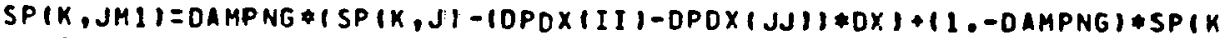
$+, J M 11$

390 CONTINUE

400 CONTINUE

OOF = AMAX $1100 F$ OUANI

IF IOUAN.GT.FERROR I IFOUT $=J X$

DO $410 I=1$, NCHANL

$P(1, J)=P(1, J M 1)+0 \times * O P O X(I)$

10 CONTINUE

PRINT OUT CROSSFLOW FORCE POINTS IF USED

641200

641300

641300

641400

641500

641700

641800

641900

642000

642100

642200

642200

642300

642400

642500

642600

642700

642800

642900

IF (J6.NE.1.AND, J6.NE O3.OR.IIERAT.GT,1) GO TO 440

IF $(J X, E 0.2)$ URITE 113,0301

NCOUNT $=0$

DO $420 K=1$, NK

IF DUM IFIXIFOIVIKI)

IFOI I INCOUNT +1$]=K$ IFOUM

420 NCOUNT $=$ NCOUNT + IF DUM

URITE $₫ 13,540)$ JX, IIFOIV(L),L $=1, N C O U N T)$

643000

643200

643300

643400

643500

643600

643800

643800 GO TO 440

430 WRITE $(I 3,500) J \times, x(J \times)$

WRITE $\mid 13,510)$

WPITE $(I 3,520)(I, H(I, J), F(I 1, J), P(1, J), H(I, J M I), F(I, J M I), P(I, J M 1)$, $I=1$, NCHANL)

IF IIERROR.GT.II CALL EPROR

440 CONIINUE

649000

644100

644200

644300

644500

644600

644600

RAOIAL CONDUCTION FOR RODS

644800

IFIICON.EO.DI GO TO 445

CALL CURVE $(O X 1, \times(J X-1) / Z, A X I A L, Y, N A X, 1 E R R O R, 1)$

CALL CURVE $(O X 2, X(J) 1 / 2, A X I A L, Y, N A X, I E R R O R, 1)$

TEST: $=(0 \times 2+1 . E-5) /(0 \times 1+1 \cdot E-5)$

IFITEST.LT.3..AND. TEST O.GT.0.33160 10 15

C ENDPOINTS HALVED ACCORDING TO TRAPEZOID RULE

OXSUM $=10 \times 1 * 0 \times 21 \neq 0.5$ 
DXF $=0 x / 20.0$

$\begin{array}{ll}X F & 11 \text { IDXF }=1,19\end{array}$

0011 IDXF $=1,1$
$X F=X F+D \times F$

CALL CURVE IOX, XF $/ Z, A X I A L, Y, N A X$, IERROR, 1

11 OXSUM $=$ OXSUM OX

$O X=0 \times 5 U H / 20.0$

60 TO 18

15 CONTINUE

$X$ CUMY $=A M A X)(1 X(J X)-.5 \$ 0 X) / 2,0.1$

CALL CURVE IOX,XOUMY, AXIAL,Y,NAX, IERROR, 1

18 CONTINUE

DFX $=A F L U X+O X$

441700

CALL ROOCON INROD,JX,T,HF ILH, DFX,OT)

445 CONTINUE

RADIATION INCLUDEO IN HEAT FLUX

IFIIRAD.EO.OI GO TO 449

CALL RADIATIJX)

449 CONTINUE

IF (NN.EO.1) WRITE $(13,550)$

CALL ELAPIMTIME I

WPITEII3,560I ITERAT, ISAYT, ISOUT, IFOUT,OIM, ODH, OOF, OOH, MT IME

IF IJUMP.EO.2.AND.0T.LT.1.EG) CALL HEATIO,1,1,-3)

IF IJUMP.EO.1.AND. ITERAT.NE.NTRIESI 60 TO 495

IF IJURP.NE.1) RETURN

490 CONTINUE

C

RETURN

$$
500
$$

500 FORMAT 1 44 HIERROR DETECTEO AT SUBROUTINE SCHEME AT NODE,13,6H X

650900 .

वHDG

* S D O T *

DPRT,S COBRAACFTL, SDOT

FURPUR 28R1 GA-8 E35 S74T11 05/26/81 17:37:05. 
COBRA ACFTLI1). SOOT

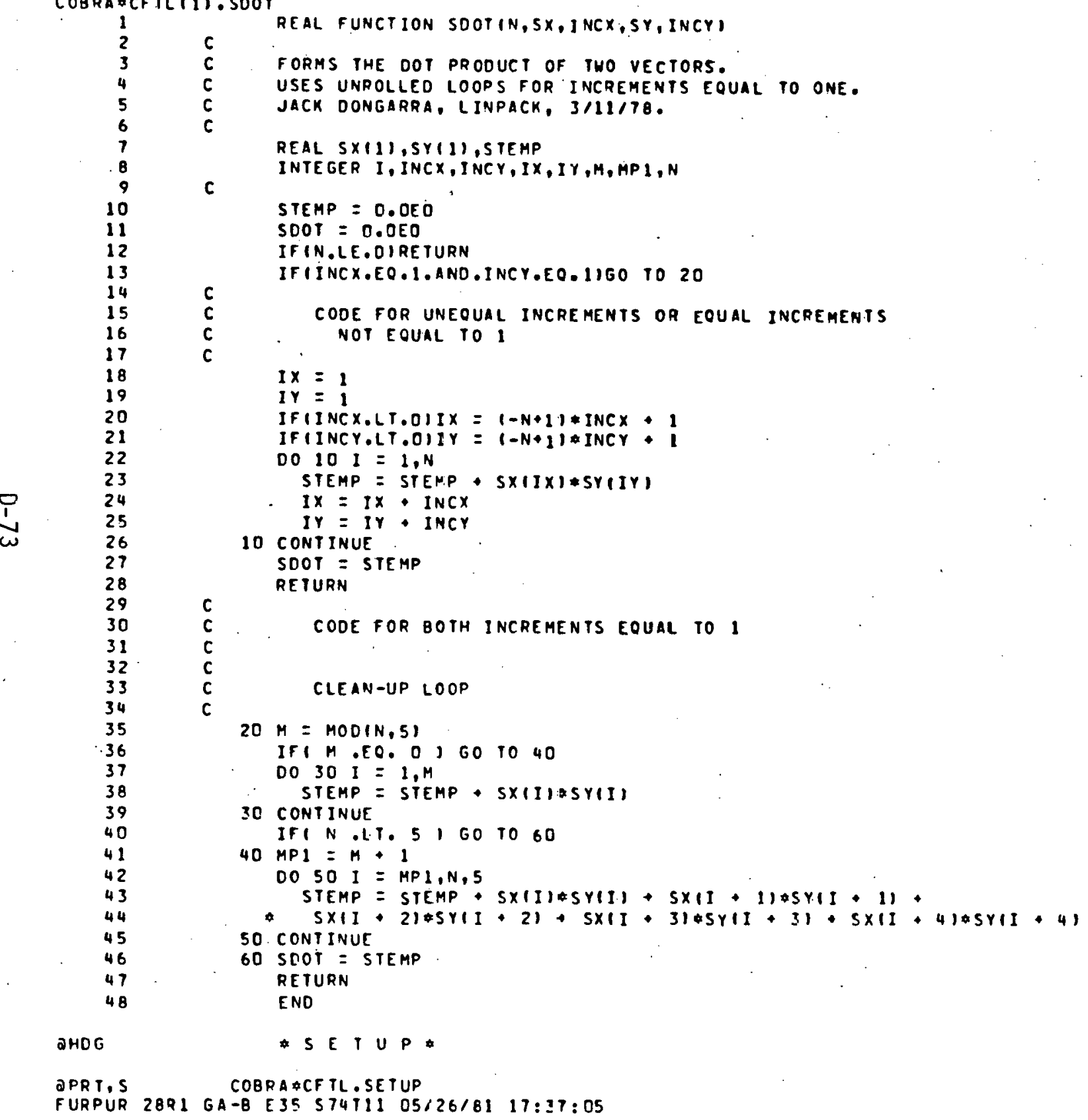


COBRA CFTLI1). SETUP

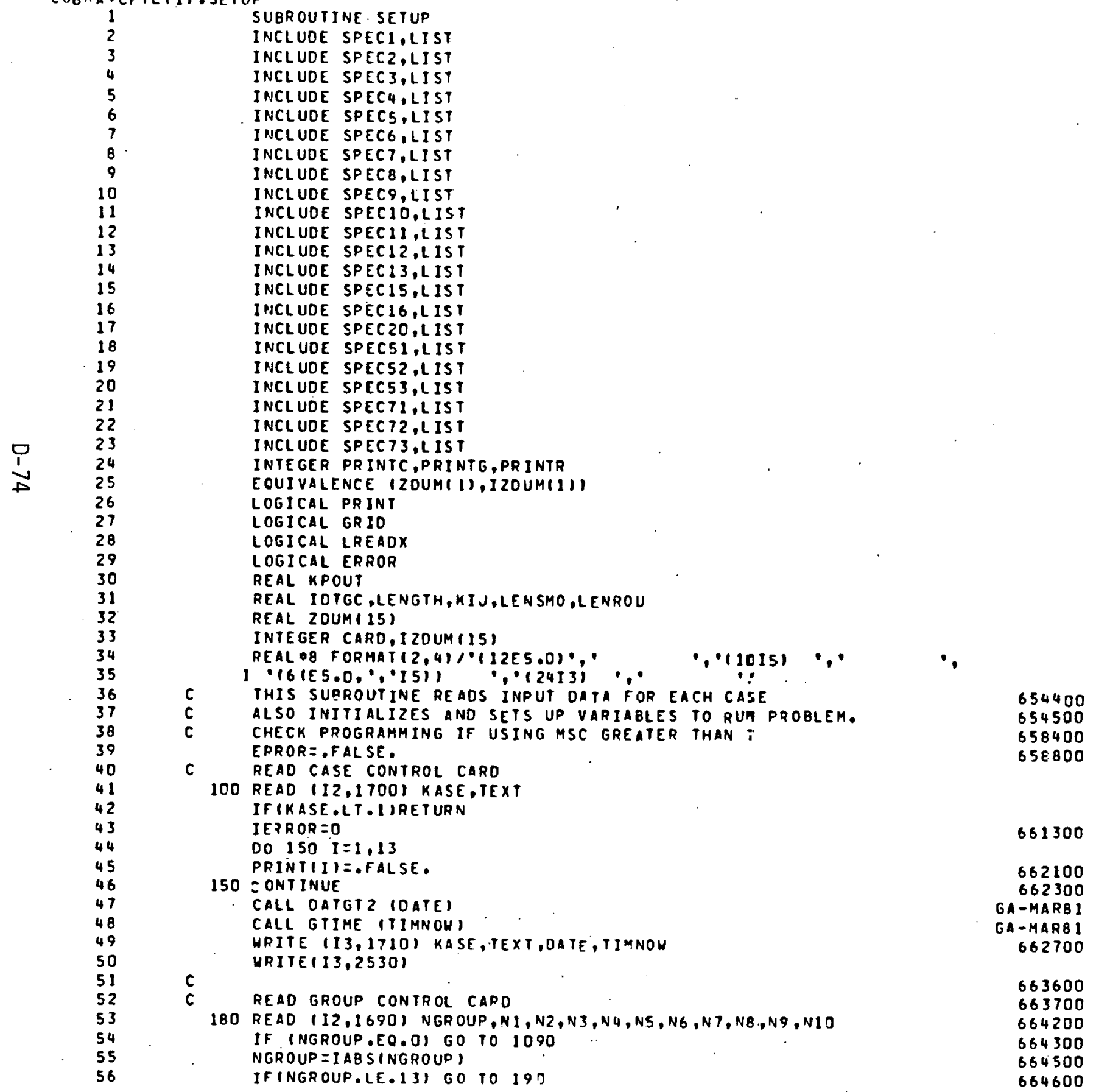


EFROR=.TRUE.

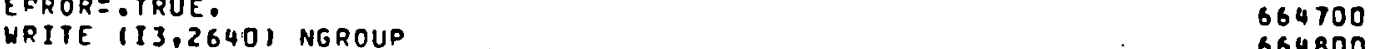

GO TO 1090 . $\quad 664800$

190. $C A R D=1$

664900

PRINTI NGROUP) $=$.TRUE.

665000

ROUP, NI,N2,N3,N4,N5,NG,N7,NB, Y9,N10

3ROUP $1,2,3,4,5,6,7,8,9,10,11,12,13 \quad 668200$ 60 TO $1200,240,250,260,470,510,550,630,740,800,820,1080,10851, \quad 666300$ - NGROUP

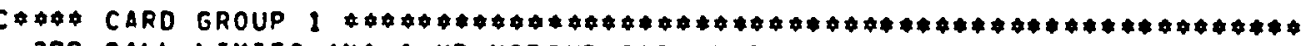

200 CALL LIMITS (N 1,1 , MP, NGROUP, CARO,ERROR)

NPROP $=M I N O$ INI, MPI

666400

666500

666600

DTHAX $=750$

666700

IF $1 N 3, E 0.1)$ REAO 112,17301 DTMAX

GO TO 180

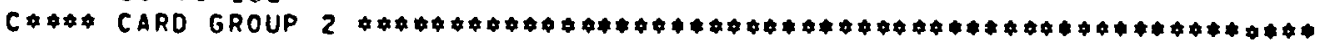

240 CONTINUE

LAMNF $=N 5$

NFH $=N 7$

$N F T=N 8$

IFINFH.GT.01 GO TO 241

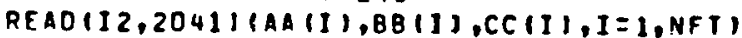

669000

669100

669200

669300

669800

669900

$241 A H(1)=0.023$

$A H(2)=0.8$

$A H(3)=0.4$

$A H(4)=0.0$

IF ILAMNF.GT.OIREADII2,2041IIAALII),BBLII),CCLIII,I=1,NFT)

670100

670300

670400

670500

670600

IF (NG.GT.O) CALL LOAD IAH,ZOUM,ZOUM,4,4,12,1,FORMAYI1,1),CARD,12) 670900 IFINFH.EO, O)GO TO 244

GO TO $1242,243,242,243)$, NFH

42 CONTINUE

READII 2,204I) (AAII),BB!I),CCII),II, NFI)

READII 2,204I) (AARII),BBRII),CCRII),I =1,NFT)

IF (NFH.EO.1)

-REAOII2,204II (HAAII), HBBIIIIHCCII),III, NFT)

R[ADII2,2041) (HAARII),HBERII),HCCRII), I=1, NFT)

GO TO 244

243 CONTINUE

READ (I2,2041) (AAII),BBII), CCII), I =1, NFT)

IF (NFH.EO.4) GO TO 244

READII2,2041) (HAA(I),H3B(I),HCCII), I =1, NFT)

671200

671300

671400

671500

671600

671700

671800

671900

672000

672100

672200

244 CONTINUE

GO TO 180

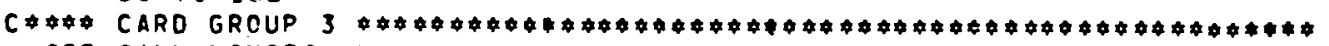

250 CALL LIMITS (N1,1, MP, NGROUP, CARD,ERROR)

NAX = MINOINI

CALL LOAO IY, AXIAL, ZOUM,NAX,N1,12,2,FORMATI1,1), CARO, I2)

IRAD $=N 2$

IFIIPAO.EO.OT 60 TO 252

CALL LIMITSIIRAD, 0,1 , NGR OUP, CARD, ERROR

CALL RADRED (N3)

CALL LIMITS INSF, I, NU, NGROUP, CARD, ERROR I

252 CONTINUE

GO TO 180

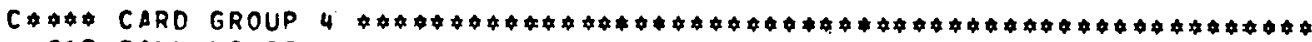

260 CALL LIMITS IN2, I, MC, NGROUP, CARO, ERRORI NCHANL $=M I N O I N 2, M C I$

672700

672800

672900

673000

673100

673200

673300

673400

673500

673600

673700
673900

674000

674100

674200 
CALL LIMITS (N1,1, N2, NGPOUP, CARD,ERRORI

674300

NHK $=$ MAXC $(N 4,0)$

NHKK $=N W K$

IF INWK.EO.O) GO TO 270

674400

674500

674600

674700 CALL LIMITS INWKK, $1, H H, N G R O U P, C A R D, E R R O R I$

674800

$270 N S=M A X O(N 3, D)$

NSS $=$ NS

IF INS.EO.O) 60 TO 280

CALL LIMITS INSS, I, MG, NGROUPI, CARD, ERROR I

674900

675000

675100 NS =MINOINSS, MGI 1, MG, NGROUPI, CARO, ERRORI

675200

675300

675400

675500

IF $(N 5 \cdot G T \cdot O)$ LUEN5

$J=0$

NK $=0$

$290 \mathrm{~J}=\mathrm{J} \cdot 1$

IF (J.GT.NI) GO TO 320

REAO (LU, I 750$) \mathrm{N}, 1,(120 \cup M(K), K=1,15)$

675600

675700

675800
675900

675900
676000

676100

6762.00

$C A R D=C A R D+1$

IF (J.GT.NCHANL) GD TO 290

CALL LIMITS II, I, NCHANL, NGROUP, CARD, ERROR )

676300

676400
676500

I=MINOII, NCHANL

NTYPEIII $=N$

$A C(1)=2 D U M(1) * 1 . E-6$

PHIII=ZOUHI2I*1.E-

$00310 \quad L=1.4$

$L 1=3 * L+1$

L2=IABSIIZOUMILIN

CALL LIMITS IL2, O, NCHANL, NGROUP, CARO, ERPORI

L2EMINOIL $2, N C H A N L I$

IF $(L 2 \$(L 2-1), L E .0)$ GO TO 300

$N K=N K+1$

3OO CALL LIMITS INK, 1,MG,NGROUP, CARO,ERRORI

LCIIILIEISIGNILI,I ZOUMILIII

GAPSII,L $=$ ZOUM $(L I+11 * 1 \cdot E-3$

310 CONTINUE

GO TO 290

320 ATOTAL $=0$

NK $=0$

DC $350 \quad I=1$, NCHANL

$00330 \quad L=1,4$

IF ILCII,LI.EO.OU GO TO 330

$J=I A B S(L C I 1, L)$

IF IJ.LE,I I GO TO 330

$N K=M I N O(N K+1 ; M G)$

$J K(N K)=J$

IK $(N K)=I$

GAPN (NKI I GAPS I I L

GAP $(N K, 1)=G A P N(N K)$

LENGTHINK I $=$ OISTII,L)

330 CONTINUE

IF IPWIII.GT.O..AND.ACIII.GT.0.1 GO TO 340

EFROR= .TRUE.

WRITE 113,26501 NGROUP

677100

677200

677300

677400

677500

677600

677700

677800

677900

678100

678400

678500 .

678700

678800

678900

679000

679100

679200

679300

67900

679700

679800

680000

680200

680300

680500

680600 
OHYONIII $=$ OCII)

681000

OHYOII, II =OHYONIII

681200

681400

$\begin{array}{ll}\text { IF } I N K . E O .0160 \text { TO } 410 & 681500 \\ 00380 K=1, N K & 681600 \\ I I=I K I K) & 681700\end{array}$

$\begin{array}{ll}\text { IF } I N K . E O .0160 \text { TO } 410 & 681500 \\ 00380 K=1, N K & 681600 \\ I I=I K I K) & 681700\end{array}$

$I I=I K|K|$

$J J=J K(k)$

LOCA $(1, K)=K$

LOCA (NAAAP $1, K)=1$

681800

681900

681900

682000

IF $(K, E O . L)$ GO TO 380

682100

682200

682300

682.400 $j=J K$ IL

IF III.EO.I.OR.II.EO.J) 60 TO 360

682500 If (JJ.EO.I.OR.JJ.EO.J) GOTO 360

682600

682700

68280

682800

IF INAO.LT.NAAAP 1 G GO 10370

682900

683000

683100

683200 WRITE $\mid 13,27401 K$

GO TO 1680

370 LOCA (NAD,K):L

LOCA $(N A A A P 1, K)=N A D$

683300

$L=N A A H P I * N C H A N L$

$$
\text { DO } 390 \quad I=1, L
$$

390 IL OC $111=0$

IF (NK.EO.O) GO TO 410

683400

683500

683600

683600

683700

683800

683900

684000

684100

684200

I I $=$ I IK (K) -1 I ONAAHP I

584300

58400

684400

$j=J j+N A B H$

NAOI $=I L O C(I)+1$

684500

684600

684700

684800

684900

685000 ERROR =, TRUE.

WRITEII3,2750) NADI, NAOJ,MI

9 CONTINUE

$I L O C(I I+N A O I)=K$

ILOCIJJ+NADJ) $=K$

685100

685200

685300

685400

685500

685600

685700

410 IF INWK.EO.OI GO TO 430

DO $420 \mathrm{~K}=1$, NHKK

READ $(12,1800) \mathrm{KH},(Z O U Y(I), I=1,6)$

685900

IF (K.GT.NHK) GO TO 420

685900

686000

686100

686200 KHEM INOIKW, NWK )

RHOLCPIKWI $=2 O U H 11$

686300 
IKH(KH) $=1200 M(3)$

RHALLI1,KW) $=20$ UM 141

686500

$J K W(K W)=\operatorname{IZDUM}(5)$

RWALL $(2, K H)=2 D U M(6)$

686600

686700

686800

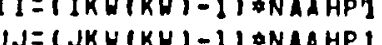

686900

687000

687100

687200

687300

687400

687500

687600

687700

687700

687800
687900

687900
688000

688100

688200

688300

688400

688500

688600

688700

688800

688900

689000

689100

689200

689300

689400

689500

689600

689700

689800

689900

690000

6.90100

690200

690300

690500

690600

690700

690800

690900

691000

691100
691200

691300

691400

691500

691600

691700

691800

691900

692000

692100

NAXL =MINDINZ, MLI

CALL LIMITS INI, O, MA,NGROUP, CARD,ERRORI 
NAFACT = MINOINI, MAI

IFINZ.LE.OI GO TO 180 CALL LOAD IAXL, ZOUM, ZOUM, NAXL,N2,12,1,FORMATI1,1),CARD, 121 692400 DO $490 \mathrm{~J}=1$, NAFACT

REAO (12,1690)

CARD $=C A R O+1$

CALL LIMITS II I NCHANL NGROUP , CARD ERPORI

I =MINOII, NCHANLI

ICAREAIII =J

NCHIJI $=I$

CALL LOAO IAFACTII,JI,ZOUM,ZOUM,NAXL, NAXL,12,1,FORMATI1,11,CARD, I - I

$490^{\circ}$ CONTINUE

IF INI.EO.NAFACTI GO TO 1.80

$N 2=N 1-N A F A C T$

DO $500 \mathrm{~J}=1, \mathrm{~N} 2$

READ (12,1760) I, (ZOUHIL),L=1,12)

500 CONTINUE

GO TO 180

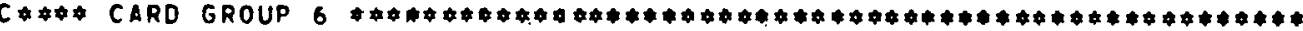

510 IF I (NK-I) \& (NK-MG) .LE.OI GO 10520

ERROR=.TRUE.

WRITE 113,2670$)$ NGROUP

GO TO 1680

520 CALL LIMITS IN $2,0, M L, N G R O U P, C A R D, E R R O R$ )

NGXL $=$ MINOINZ, MLI

CALL LIMITS INI, O, MS, NGROUP, CARO,ERRORI

NGAPS $=M I N O(N I, M S)$

IFIN2.LE.DI GO TO 180

CALL LOAO IGAPXL,ZOUM, ZOUM,NGXL, N2,12,1, FORMATI1, 11, CARO, 121

DO $530 L L=1$, NGAPS

READ $112,16901 \mathrm{~K}$

$C A R O=C A R D+1$

CALL LIMITS IK, I, NK, NGROUP, CARO, ERROR

$K=M I N O(K, M G)$

$I D G A P(K)=L L$

NGAP(LL) $=K$

$L=L L$

CALL LOAD IGFACTI1,L1, ZOUM,ZOUM,NGXL, NGXL,12,1, FORMATI1,11,CARD, IZ $+3$

530 CONTINUE

IF INI.EO.NGAPSI GO TO 1.80

N2 $=$ N1 - NGAPS

DO $540 \mathrm{~J}=1, \mathrm{N2}$

REAO $112,16901 K$

READ (12,1730) (ZOUMII), 1=1,12)

540 CONTINUE

GC TO 180

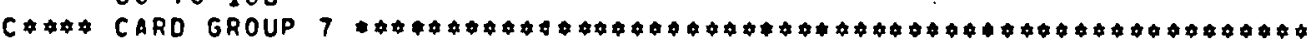

$550 \mathrm{~J} 6=\mathrm{N} 1$

IFIN7.LE.CI N $7=N C H A N L$

IF (NT.GT.NCHANL) N7 =NCHANL

NRAMP = MAXOIN5, 11

NARAMP = MAXJ 11, NARAMP

GRIO =.FALSE.

IF $((J G-1)$ (J) JS-3).GT.0) GO TO 180

IF IINCHANL-1) INCHANL-MC),LE.OI GO TO 560
692500

692500

692600

692700

692800

692900

693000

693100

693200

693300

693400

693500

693600

693700

693800

693900

694000

694100

694100

694200

694300

694400

694500

694600

694700

694800

694900

605000

695000

695100

695200

695300

695400

695500

695600

695700

695800

695800

695900

696000

696100

696200

696300

696400

696500

696600

696600

696700

696800

696900

697000

697100

697200

697300

697400

69750

697500

697700

697800

697900 
698000 WRITE $(13,2660)$ NGROUP

698100

698200

CALL LIMITS IN3,O, MZ,NGROUP, CARO, ERROR)

698300 NGRID $=M$ INO IN $3, M Z$ )

IF $(J 6 . E Q .2) 60$ TO 590

PITCH = PITCH 1 $E-3$

698700

DIACH:PITCH:

DIA DIA I.E-

THICK $=$ THICKDI.E-3

LU $=I 2$

IF ING.GT.DI LUENG

DO $570 \quad M=1, N 2$

READ $(L U, 2240) K,(Z O U M(I), I=1,3)$

$C A R O=C A R D+1$

CALL LIMITS ( $K, 1, M G, N G R O U P, C A R D, E R R O R$ )

698800

$K=M I N D(K, M G)$

DUR $(K)=20 U M(1)$

$X$ CROSS $(K, 1)=Z$ OUM 12

$X$ CROSS $1 K, 2:=2 D U M 13$

570 CONTINUE

CALL LOAD INHRAP, I ZOUM, IZOUM, N7,N7,10,1,F ORMATI1, 2), CARD, - LU)

DO $580 \quad I=1, N C H A N L$

SBO NURAPS (I) NURAPIII

IF $1 \mathrm{J6} . E 0.31 \mathrm{GO}$ TO 590

60 TO 180

$590 N 4=I A B S(N 4)$

CALL LIMITS IN4, I, MK, NGROUP, CARO,ERRORI

NGRIDT $=$ MINO(N4, MK)

MIN $=$ NGRID

CALL LOAD IGRIDXL, IGRID, ZOUM, MIN,N 3,12,2, FORPATI1,31, CARO I

DO 595 I $=1$, NGR IOT

REAOII2,2041) COC1111,COC21I1, COC311),COC4111

595 CONTINUE

READI12,2050) SH,RSC,SSC

$S H=S H \$ 1, E-3$

DO $620 \quad I=1, M 4$

DO $610 \quad I I=1$,NCHANL

READ (12,2260) J,ZOUM(1),K,ZOUM(2)

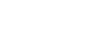

699200

699300

699500

699500

699600

699700

699800

699900

730000

100100

700200

700400

700500

700600

700700

700800

701000

701100

701200

701300

701400
701500

701600

701700

READ (I2,2260) J,ZOUH(1),K,ZOUM(2)

701800

IF II.GT.NGRIDT) 60 TO 620

CALL LIMITS (J, I, NCHANL, NGROUP, CARO,ERROR)

JEMINOI J,NCHANL)

CD(J,I) = ZOUM(I)

IF $(K . L E .0) \quad 60$ 10 610

CALL LIMITS (K, I,NK, NGROUP, CARO,ERROR)

$K=M I N D(K, N K)$

$K=M I N O(K, N K)$
$F X F L O H, I)=20 U M(2)$

610 CONTINUE

620 CONTINUE

GO TO 180

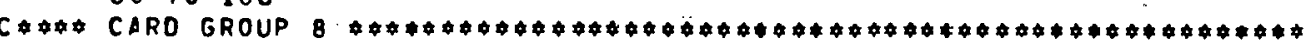

630 CALL LIMITS (N2,1, MR, NGROUP, CARD,ERROR)

702800

702900

103000

703100

703200

703300

703400

703600

703600

703700
703800

703800
703900

704000

704100

704200 NFOD $=$ MINO (NZ, MR) 
$N C=\operatorname{MAXOIN3,0)}$

IF ING.EO.4I ICON=1

704500

$L U=12$

IF (N9.GT.O) LUENQ

705900

706100

706200

CALL LIMITS IN1,1,N2,NGROUP,CARD,ERRORI

MSC $=\operatorname{MAXO}(N) D, 6)$

DO $660 \mathrm{~J}=1, \mathrm{N1}$

READ (LU,1799) I, IZOUM(L) 1,L1=1,19)

706400
706600

$C A R D=C A R D+1$

$O R(1)=20 U M(11 \% 1 . E-3$

RADIAL III $=204 M 121$

DO $650 \quad L=1,6$

PHIIIOLI=2OUMI $2 * L \cdot 21 \quad 707500$

CONIINUE

707500
707600

707700

707800

REAOILU,1801) LRII,T),PHIII,7)

652 CONTINUE

$D(I)=O R I I)$

708000

660 CONTINUE

TREF $=0$.

708500

709000

739100

IF $(N C . G T .0) \quad 6010661$
$R F(1)=0$.

GO TO 180
READ 112,1770$)$ (RF (J).

READ (12,1770) (XKF $J j, J=1,5)$

REAO $112,17701, H G A P A J i, j=1,5$

REAO 12,17701 (HGAPXJ), $J=1,41$

READ $(12,1770)$ (OPFRAC $(J), J=1,5$ )

REAO $(12,1770)$ (RHOFIJ), J=1,5)

IFIICON.NE.II GO TO 280

NSF $=$ NU

READ 112,1690। NCONR

$\because A R D=C A R D+$.

IFINCONR.EO.0I GO TO 683

CALL LIMITSINCONR, $1, N P O D$, VGROUP, CARO, ERRORI

711500

711600

READ (I2,1690) IICONR(I), I =1, NCONR)

OC 682 I $=1$, NCONR $\quad 712000$

682 COLL LIMITSIICONRIII, 1, NRDO, NGROUP, CARD, ERROR I

712100

CONTINUE

GO TO 180

683 CONTINUE

DO $684 \quad I=1, N R O D$

ICONRIII =

712300

712400

712500

712600

712700

$\begin{array}{ll}\text { NCONR }=\text { NROD } & 712800 \\ \text { GO TO } 180 & 715400\end{array}$

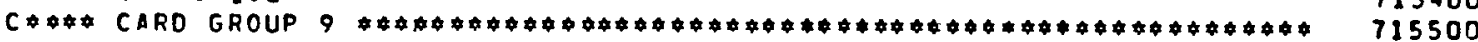

740 NSKIPX $=$ MAXO(NI,1)

NSKIPT $=$ MAXOIN 2 , 11

READII 2,17901 Z, TIIME, WEFRX, WERRY, FERROR, KI J, SL, FTM , THE TA, USDON, O

- AMPNG, ACCELY, ACCELF

AMPNG, ACCELY,
CARD $=$ CARD+1

716600

$12=C A R D$

READ 12,16911 NOX, NDT, NTFIES, ITFY, ITRYM, LENSMO,LENROU

716700

718000

718100 
LENSMO $=$ LENSHO $/ 1000$

LENROU $=$ LENROU $/ 1000$

$C A R D=C A P D+1$

CALL LIMITS INOX,1,MX-1, NGROUP, CARD,ERROR )

718300

NOXP I = NDX I

If ISL.LT.J.OE-5) $S L=0.5$

ELEV $=$ COS ITHETA $\$ P I / 180$. I

IF INTRIES.LT.11 NTRIES $=20$

IF (FERROR .LE - 1.E-5) FERROR=0.01

IF IITRY.LT.1) ITRY =MAXO (2\$NK, 20)

IF IITRYM.LT.1) ITRYM =5

IF IKIJ.LE. L.E -5) KIJ $=0.5$

IF (WERRY.LE.1.E -5) HERRY $=0.001$

IF (WAMPNG.LE.1.E-5) DAMPNG $=0.0$

IF (DAMPNG DLE I.E-5) DAMPNG $=0.8$

IF (ACCELF.LE.1.E-5) ACCELF $=1.0$

IF (ACCELY ILE $1 . E-5)$ ACCELY $=1.6$

LREADX =.FALSE.

IF INT,EO.1) LREADX =.TRUE.

IFI.NOT OREADX) GO TO 775

READ $2041,(X) J,, J=1, N D X)$

READ 2041 ,

DO $774 \mathrm{~J}=1$, NDXP1

$x(J)=x(J / / 1000$.

774 CONTINUE

$O X=|x(2)-x(1)|$

775 CONTINUE

$Z Z=2$
$Z=Z 11000$

C FIND RELATIVE AXIAL LOCATION WHERE HEATING STARTS

DO $776 \quad I=1$, NAX

IFIAXIALII).6T.0.051 GOTO 777

776 CONTINUE

$X H=Y(N A X)$

GO 10779

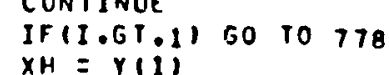

$X H=Y(1)$

GO $10 \quad 779$

778 CONTINUE

$X H=Y \mid I-1)$

779 CONTINUE

$X H=X H \cdot 2,10$ TO 795

IF (LREAOX) GO TO 795
$O X=$ ZIFLOAT(NOX)

DO $794 J=1$, NOXP1

$x(J)=D X(J-1)$

794 CONTINUE

795 CONTINUE

DT $=1 . E 10$

IF I NOT.GT . O. AND.TIIME.LE.O.I NDT $=0$

IF INOT.GT.OI OT: TTIME/FLOATINDTI

SAVEOT $=D T$

IF I.NOT. LREADX) DXX $=O X$

If $\{2 . G T .0 .160$ TO 180

WRITE $(I 3,2690)$ NGROUP, I 2

ERROR =. TRUE.

718400

718500
718600

718700

718800

718900

719000

719100

719200

719300
719400

719500

719600

720000

720100

720200

720300
720400

.720700

720900

721000

721100

721200

721300

721500

721600

721700

721800

721900

722000

722100

122200

722300

722400

722500

724100
724200

724300

724400

724500

724600

724700

724800

724900
725000

725300

725400

725500 


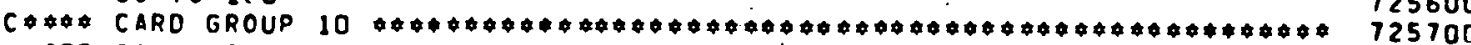

800 CALL LIMITS IN1, 0, 4, NGROUP, CARD, ERRORI

NSCBC = MINOINI, 4I

$J S=N 3$

725800

READ 112,17301 ABETA,BBETA

725900

CARD $=C A R D+1$

IF $(J 5 \cdot E 0.0) \quad G K=0$.

ABETA 726300

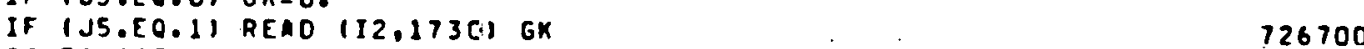

GO $10180 \quad 726800$

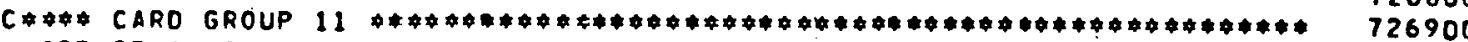

820 IF IINCHANL-I) INCHANL-MC J.LE.0) $6010830 \quad 727000$ EPROR= -TRUE.

WFITE $(13,2660)$ NGROUP

GO TO 1680

830 IF (INPROP-1) * (NPROP-MP) .LE-O) GO TO B4O EPROR = TRUE

EPROR = ITRUE

GO TO 1680

727100

727200

727200

727300

727400

727600

727700

840 IH:IABSINII
IG IABSINZI

727800

NP $=M I N O(M A \times O(N 3,1), M P)$

IF (NP.F.Q.1) GO TO 860

CALL LIMITS IN3, 1, MP, NGROUP, CARO,ERRORI

727900

860 NHEMINOIMAXOIN4, 11, MPI

If (NH.EO.1) GO TO 870

CALL LIMITS IN4,1, MP, NGROUP, CARD,ERRORI NHEMINO(N4, MP)

87.0 NG = MINDIMAXO(N 5, 11, MP)

IF ING,EO,II GO TO 880

CALL LIMITS IN5, 1, MP, NGRO JP, CARD, ERRORI NG $=M I N D$ (N5, MP)

880 NOEMINOIMAXOING, 11, MP)

IF (NO.EO.1) 60 TO 890

CALL LIMITS ING,1, MP, NGRO JP, CARD,ERROR

729300

729400

729500

729600

729700

729800

129000

729900

730000

730100

730200

730300 NCEMINO (NG, MP)

$890 \times 10=N 7$

NHX $=M I N O(M A X O(N .9,1), M P)$

IF INHX $E 0,11$ GO 10 OOD

CALL LIMITS INB, 1, MP, NGROUP, CARO,ERROR

730400

730500

730600

730700

730800

730900

731000

900 READ $(12,1770)$ PEXIT,HIN,GIN, AFLUX PEXITISPEXIT०1000

GIN $=$ GIN*1000.

$C A R D=C A P D+1$

HIN $=A B S(H I N)$

PREF =PEXIT

731200

731900

PREF $=$ PEXI
$T F=250$.

732000

IFIIH,EO.1 OOR. IH,EO.3) TF $=H I N-1$.

CALL PROP $11,1,1,1, N C H A N L$

IF IIERPOR.LT.II GO TO 910

EPROR = .TRUE.

WRITE $(13,2710)$

IEFROR $=0$

910 CONTINUT

IF IIH.GE.21 GO 10960

732200

732300

732400

732500

732600

732700 
930 TIN $=$ HIN

733700

CALL CURVE IHIN, IIN,HGPT, TPOUT, NPROP, IERROR, 11

$\begin{array}{ll}94000950 I=1, N C H A N L & 734100\end{array}$

950 HINLET IIIZHIN $\quad 734200$ 734300
73400

960 CRLL LOADIHINLET, ZDUM, ZOUM,NCHANL, NCHANL, 12,1,FOR

- MAT 11,11, CARD, I2)

IF IIH.LI.3I GO TO $990 \quad 734600$

$\begin{array}{ll}\text { DO } 980 \quad I=1, N C H A N L & 734700\end{array}$

CALL CURVE I'VOUT, VIN, HGPT, TPOUT, NPROP, IERROR,, 1)

HINLETIIII=VOUT

980 CONTINUE

$9900010101=1, N C H A N E$

VIN $=$ HINLETII)

CALL CURVE IVOUT, VIN, TPOUT,HGPT, NPROP, IERROR, 11

735600

735700

735700 TINLETIII = VOUT

736500

IF I I.EO.1) TIN = TINLETII)

1010 TINEAMINIITINLETIII, TIN

IF IIERROR.LT.1I GO TO 1020

ERROR= .TRUE.

736900

737000

WFITE $\mid 13,2720)$

IERROR $=0$

1020 CONTINUE

CALL SPLIT |IG,GIN I

DO $10501=1$, NCHAN

$1050 \mathrm{FINLETIII=F(I,I)}$

IF (IG.LT.ZI GO TO 1070

CALL LOADIFINLET, ZOUH, ZOUH, NCHANL, NCHANL, 12,1, FORMATI1,11, - CARD, I 21

DO 1 C6O I = I, NCHANL

1060 FINLET III $=$ GIN ATOTAL OF INLETIII

1070 IF (NP.GT.1) CALL LOAD IYP,FP, ZOUM,NP,NI, 12, Z, FORMATI1,1), CARO, I2) IF INH.GT,1) CALL LOAD IYH,FH,ZDUM,NH,N4,12,Z,FORMATI1,11,CARD, 121 IF ING.GT, II CALL LOAD IYG,FG,ZDUM,NG,N5,12, 2, FORMAT 11,11, CARO, I2

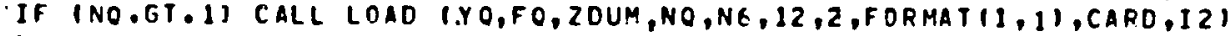
IF INHX,ST.1) CALL LOAD IYHX,FHX,ZDUH, NHX,NE,12,2,FORMATI1,1),CARD 1,121

GO TO 180

C

1080 NOUT $=$ N

NPCHAN $=$ MAXOIN2,0)

NPCHAN = MINOINPCHAN, N2, MC

NPROD $=4 A X O(N 3,0)$

IF INPRCD.NE.O). CALL IIMIIS IN3,1, MR, NGPOUP, CARD, ERROR )

NPROD $=M$ INO (NPROD,N3, MR)

NFGAP $=$ MAXOIN 5,0$)$

IF INPGAP.NE.O) CALL LIMITS (N5, I, MG, NGROUP, CARO, ERROR)

NFGAP = MINO INPGAP, N $5, M G$ I

IF INPCHAN.GT.OI CALL LOAO IPRINTC,ZOUM, ZDUM,NPCHAN,N2,24,1, FORMA $+(1,4),(A R D, I 2)$

IF INPROD.GT.OI CALL LOAD IPRINTR,ZDUM,ZDUM,NPROD,N3,24,1,FORMATI

$(1,4), C A P D, 121$

737100

737200

738200

738500

738700

739000

739200

739300

739400

739500

739600

739700

739800

739900
740000

740100

740200

740300

740400

740500

740800

740900

741000

741800

741900

742100 
2041 FCRMAT (6E10.5)

2050 FORMAT IBE 10.51

$\begin{array}{ll}2120 \text { FORMAT ISII5,F5.011) } & 752300\end{array}$

2240 FORMAT $115,10 E 5.21 \quad 752700$

2260 FORMAT IIS,ES.2,15,E5.2)

2380 FORMAT 19E5.21 753000

2530 FORMAT I25HOSUMMARY OF INPUT OPTIONS/56H GROUF NI N2 N3 N4 753100

2530 NG N7 NB N9 N10I
NE NG N2 N3 N4 753100 2640 FORMAT I'IILLEGAL GROUP IDENTIFICATION. IMPOSSIBLE TO CONTINUE EOI
TIING. CASE WILL BE ABORTEO. NGROUP=? I5I

2650 FORMAT POERROR CONOITION EXISTS IN EITHER PHIII OR ACIII WITHIN C 753600 TARD GROUP D I5I

2660 FORMAT 1 O O\$NCHANL HAS NOT BEEN DEFINED FOR GROUP, $159^{\circ}$. EDITING 753800 - HILL BE TERMINATED. $" 1 \quad 753900$ 2670 FORMAT 1 OOA \$NK HAS NOT BEEN DEFINED FOF GROUP, $150^{\circ}$. EOITING WIL 754000 TL BE TERMINATED."' $\quad 754100$ 2690 FORMAT 1 OOILLEGAL INPUT FOR 121 IN CARD GROUP $\because$ I5, CARO NUMBER 754400
+ I5) 2700 FORMAT 1 OCAUTION---NPROP HAS NOT BEEN DEFINED FOR GROUP $159^{\circ}$ ED 754600 -ITING WILL BE TERMINATED.',

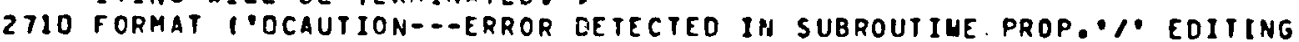
- WILL CONIINUE, EXECUTION WILL BE ABORTED.'1

2720 FORMAT IOOCAUTION---ERROR DETECTED IN SUBROUTINE CURVE. $\%$ EOITING - WILl CONTINUE, EXECUTION WILL BE ABORTED.'1

FORMAT 100AUTION---ERROR(S) HAVE BEEN DETECTEC NITHIN THE INPUT 5 755200 -TREAM. $\%$ EOITING HAS BEEN COMPLETED AND THE IMPUT WILL BE PRINTED - HOWEVER, THE EXECUTION WILL BE ABOFTED.・1

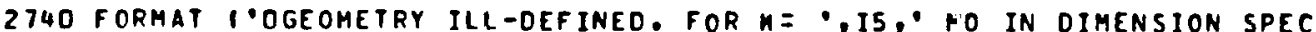
IFICATION IS TOO SMALl. ')

2750 FORMATIOOPREMATURE TERHINATION FROM CARO GROUP $\%$

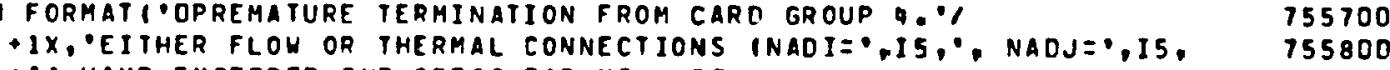
$\because$ have eXceeded the sPeCs for MI=?.151

ENO

754600

754700

754800

754900

755300

755400

755500

755900

756300

JHOG

* SE TUP 2 *

QPRT,S COBRA $\$$ CFTL.SETUPZ

FURPUR 28R1 GA-B E3E ST4T11 05/26/81 17:37:07 
COBRA OFTL11). SETUP?

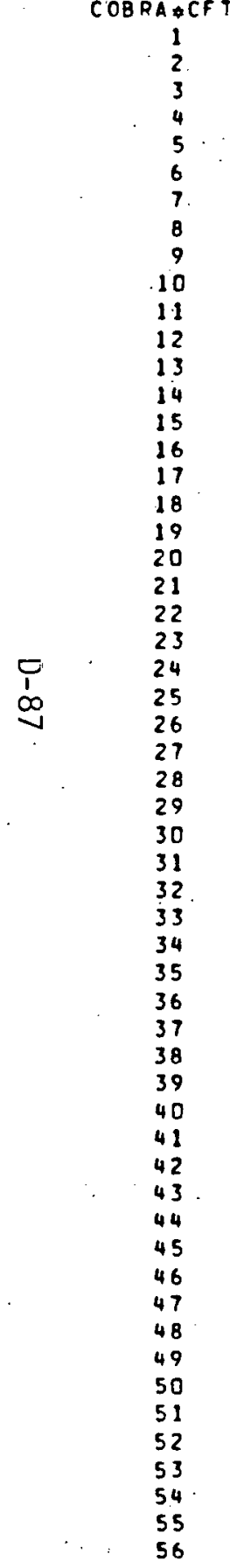

SUBROUTINE SETUP

INCLUDE SPECI,LIST

INCLUDE SPEC2,LIST

INCLUDE SPEC3,LISI

INCLUDE SPECU,LIST

INCLUDE SPEC5 LIST

INCLUDE SPECG, LIST

INCLUDE SPECT,LIST

INCLUDE SPEC1O,LIST

INCLUDE SPEC11,LIST

INCLUDE SPEC12,LIS

INCLUDE SPEC13,LISI

INCLUDE SPEC15,LIST

INCLUDE SPEC16,LIST

INCLUDE SPEC2O,LIST

INCLUDE SPEC51,LIST

INCLUDE SPECS2,LIST

INCLUDE SPEC53,LISI

INCLUDE SPECTI,LIST

INCLUDE SPEC72,LIS

INCLUOE SPECT3,LIST

LOGICAL PRINT

LOGICAL GRID

REAL KPOUT

REAL IOTGC,LENGTH, KIJ,LENSMO,LENROU

C PRINTS INPUT DATA FOR EACH CASE.

CARD GROUP 1

IF I.NOT.PRINTI11) GO TO 1260

PREF $0=P R E F / 1000$.

WRITE (I3,2620) PREFO

HRITE II 3,26221

DO $1240 \mathrm{NN}=1$, NPROP

OUTPUT $(1)=$ TPOUT(NN)

OUTPUT $(2)=$ HGPT (NN)

OUTPUT ( 3 ) =CPPOUT (NN)

OUTPUT $(4)=$ VPOUT (NN)

OUTPUT (5)=UPOUTINN

OUTPUT (6)

OUTPUT ( 7$)=$ UPOUT (NN) \#CPPOUT(NN) /KPOUT INN

HRITE II3,2630I IOUTPUTIII,I=1,7

1240 CONTINUE

CARO GROUP 2

1260 IF I.NOT.PRINTIIII 60 TO 1290

IF CNFH.EO.OI URITE 113,19201

DO $1280 \mathrm{~J}=1$, NF T

If (NFH.EO.O) WRITE $(I 3,1930)$ J,ABIJ),BBIJ),CC(J)

IF ILAMNF.GT.QI HRITE II 3,1940) J,AAL(J),BBL(J),CCLIJ)

1280 CONTINUE

IFINFH.EO.OIWRITE $(13,1950)$ (AHII), I $=1,4$

IF (LAMNF.GT.O) WRITE IIZ,1941)

IFINF.H.EO.OIGO TO 1284

1282 CONTINUE

HRITE(I3,1919)

DO $1283 \mathrm{~J}=1$, NFT

IF ( INFH-1) (NFH-3) ,EO.0)

-WRITE(I3,1929) J, AA J),BB(J),CCIJ),J,AARIJ),BBR(J),CCR(J)

760500

760600

760700

760800

760900

161000

761300

761400

761500

761700

762700

763200

763300

763500

763600 
IF(INFH-2) $(N F H-4)$.EO. O) URITE(13,1929) J,AA(J),BB(J),CC(J),J 763700 IF (INFH-2I\#(NFH-4).EO. O) WRITE II3,1928) 763800

1283 CONTINUE

WRITE 113,19511

763900

DO $1281 \mathrm{~J}=1, \mathrm{NF}$

IFINFH.EO, I)

-WRITE(I3,1952) J,HAAIJ!,HBB(J),HCC(J),J,HAAR(J),HBBR J), HCCR(J)

764000

IFINFH.EQ.2) WRITE(I3,1952) J,HAA(J),HBB(J),HCCIJ),J

IFINFH.EO.2) WRITE(I3,1949)

IF(NFH.EQ.3) WRITE(I3,1948) J,J,HAAR(J),HBBR(J),HCCR(J)

IFINFH.EO.4) NRITE(13,1947)NFT, NF T

IF (NFH. E0.4) 60 10 1284

1281 CONTINUE

C CARD GROUP 3

1290 IF I.NOT.FRINT131) GO $10 \quad 1300$

$X H O=X H \$ 1000$.

WRITE $(13,1740) \times H O,(Y(I), A X I A L(I), I=1, N A X)$

IF(IRAD.EO.1) WRI TE(I3,1742)

764200

764300

764400

764500 CARD GROUP 4

I3OO IF P.NOT.PRINT141) GO TO 1320

764800

764900

764900
765000

766100

WRITEII3,1810)

DO $1301 I=1$, NCHANL

$A C O U T=A C(T): 1 . E 6$

PHOUTEPHII 1000 .

DCOUT $=$ DCIII:1000.

$$
001302 \mathrm{~J}=1,4
$$

GAPS $(1, J)=G A P S(1, J)+1000$.

1302 DIST $(I, J)=0 I S T(I, J 1 \% 1000$.

WRITE II3,1811) I,NTYPEIII,ACOUT,PUOUT,OCOUT, ILCII,LI,GAPSII,L),

OIST $(I, L), L=1,4)$

$001303 \mathrm{~J}=1,4$

GAPS $(1, J)=$ GAPSII,J)/1000。

1303 DIST $(1, J)=015$ (I, J $) / 1000$.

1301 CONTINUE

IFINWK.LE.OIGO TO 1305

WPITEII3,1830\}

DO $1304 K=1$, NWK

WPITE (I3, 1831 , K, RHOLCP(K), WIOTH(K), IKH(K),RWALL $(1, K)$,

WIDTH(K) =W IDTH(K)/1000.

1304 CONTINUE

1305 IF (K12.LE.0) GO TO 1320

DO $1310 K=1$, NK

1310 WP(K)=ANGLE(K)\&180./PI

WRITE $(13,1850)(K$, HP $(K), K=1, N K)$

766300

766400

1320 IF (NAXL.LT.1) GO 101350

C CARD GROUP 5

IF I.NOT.PRINTI5I) GO $10 \quad 1350$ $N=1$ $N N=10$

1325 CONTINUE.

IF (NN.GT.NAFACT) NNENAFACT

IF INN , GI, NAFACT I NN=NAFACT
HRITE (I3, 1880) (NCH(J),J J,NN)

DO $1333 I=1, N A X L$ $N=N+10$ 
768000

C CARO GROUP IF (.NOT.PRINT 16$)$ GO TO 1390

768900 $N N=10$ DO $1380 \mathrm{LL}=1.6$

IF (NN.GT.NGAPS) NNENGAPS

DO $1360 \mathrm{M}=\mathrm{N}, \mathrm{NN}$

$K=N G A P(M)$

$\operatorname{IM}(H)=I K(K)$

$1360 J M(M)=J K(K)$

WRITE $(13,1890)$ (IIM(M), JM(M)),MEN,NN

DO $1370 L=1, N G \times L$

1370 HRITE (I3,2020) GAPXLIL), (GFACTIL, MI, MEN, NN I $\mathrm{N}=\mathrm{N}+10$

$N N=N N+10$

IF IN.GE.NGAPSI GO TO 1390

1380 CONTINUE

C CARD GROUP T

1390 IF 1.NOT.PRINTIII) GO TO 1450

IF $(J 6, E 0.0) \quad G 0$ TO $\$ 450$

If $(\sqrt{ } 6-2) 1400,1410,1400$

769000

769100

769200

769300

169400

769500

169600

769700

769900

770100

770200

770300

770400

770500

770600

770700

1400 PITCHOSPITCH 1000 .

DIAO =OIA 1000

THICKO = THICK $\$ 1000$.

WRITE $(13,2270)$ PITCHO,THICKO,DIAO

URITE (I 3,2280$)$ (K, IK(K), JK(K), DUR (K), IXCROSS $(K, L), L=1,2)$

$, K=1, N K)$

WRITE $(13,2320)$ (NWRAPSII), I =1, NCHANL)

171600 IF 1 J6.NE.31 GO TO 1450

1410 URITE $(I 3,2290)$ (IGRIDII), I=1, NGRIDI

WRITE $(13,2300)$ (GRIOXLII) I $=1$, NGRIO)

WRITE (I3, 2302)

DO 1405 I $=1$, NGRIOT

HRITE I 3,2301$) 1, \operatorname{coc} 1(1), \operatorname{coc} 2(1), \operatorname{coc} 3(1), \operatorname{coc} 411)$

1405 CONTINUF.

SHO $=S H+1000$

WRITEII3,2303I SHO,RSC,SSC

DO $1420 \quad L=1, N G R I D T$

1420 URITE $(13,2310) \mathrm{L},(I, C D(I, L), 1=1$, NCHANL)

If (NK.EO.O) GO TO 1450

DO $1440 I=1, N G R I O T$

II $=0$

DO $1430 K=1, N K$

IF IABSIFXFLONIK,I)I.GT.OI II =

771700

771800

771900

772000

172100

772200

772300

772400

1430 CONTINUE

IF III.EO.O) 60 TO 1440

WPITE $(I 3,2330) I$, (KK, IN IKK), JK (KK), FXFLOY(KK,I),KK=1,NK)

1440 CONTINUE

C CARD GROUP B

1450 IF I.NOT.PRINTIBII GO TC 1510

WRITE $(13,1840)$

OO $1451 I=1, N R O D$.
OROUTISORIII 1000.

772600

772700

772800

772900

773000

773100

773200

773300

773300

773600

773700

773800 
WRITE II3,1841, I,OROUT,RADIALIII, (PHIII,LI,LRII,L),L=1,MSC)

1451 CONTINJE

IF IRF (I).LT.1.E-B) GO TO 1510

774200

WRITE $(I 3,2340)$ (DFIII, I I,5

WRITE $(13,2350)$ (XKF (I), $I=1,5$ )

WRITE $(13,2355)$ (HGAP(I), $I=1,41$

WRITE 113,23601 (CPF III) I $1=1,51$

WRITE UP 13,23601 NCPFPII $I=1,5$

UPITE II3,2365) TOPFRACIII,I $1,1,5$

WRITE T13,2370) TRHOFTI, $I=1,51$

IFIICON.EO.1) WRITEII3,1741) (ICONRIII,I=1,NCONR)

C CARD GROUP?

1510 IF I.NOT.PRINTI9I) GO TO 1530

HRITEII3,2550I

WRITE 113,18601 KIJ,Z2,SL,NDX,FTM

IFIOXX.GT. O. I URITEIIJ,1861) DXX

777500

778100

ALNSKO =LENSMO* 1000 .

ALNROU $=L E N R O U * 1000$.

WPITE II 3,1862$)$ ALNSMO, ALNROU, THETA, TTIME, NOT, OT

WRITE (I3,1870) NTRIES,ITRYM,ITRY,USDON, WERPX, ACCELY, WERRY, OAMPNG, 778700 -FERROR, ACCELF

IF $10 \times X . G T .0 .1$ GO TO 1522

DO $1523 \mathrm{~J}=1$, NOXP1

$x(J)=x(J) \neq 1000$

1523 CONTINUE

PRINT $1921,(\times(J), J=1$, NOXPI)

DO $1521 \mathrm{~J}=1$, NOXP 1

$x(J)=x(7) / 1000$.

1521 CONTIVUE

1522 CONTINUE

C CARD GROUP 10

1530 IF I.NOT.PRINT(10), GO TO 1570

WRITE (I3,1990)

IF INSCBC.LT.1). WRITE $(13,1960)$ ABETA

IF INSCBC.EO.1) WRITE (I3,1970) ABETA,BBETA

If INSCEC.EO.2) WRITE $(13,1980)$ ABETA,BEETA

IF INSCBC.E0.3) WRITE $(13,2030)$ ABETA,BBETA

If INSCBC.EC.4) HRITE II3,2031) ABETA

IF (J5.EQ.1) WRITE $(13,2230) \mathrm{GK}$

C

15.70 IFI.NOT.PRINTI111) GO TO 1595

PEXITP $=$ PEXIT/1000.

GINP $=$ GIN $/ 1000$.

WRITE II3,1900) PEXITP,HIN,GINP, TIN, AFLUX

IF (HOUT.GT.D.) WRITE $(13,1910)$ HOUT

779000

779100

IF $(I H, E O, 0)$ WRITE. $(13,2460)$

IF (IH.EO.1) WRITE $(I 3,2470)$

IF (IH.EO,2) WRITE $(I 3,2480)$ (I,HINLETII), I I, NCHANL,

IF (IH,EQ.3) WRITE (I3,2490) (I,TINLETII),I:I, NCHANL)

IF (IG.EO.O) WRITE $(13,2500)$

IF (IG.EO.O) WRITE $(13,2500)$ (I 1 .EQ.1) WRITE $(13,2510)$ (I,FINLETII), I =1, NCHANL)

If $(I G . E 0.2)$ WRITE $(13,25201$ (I, FINLETIII,I=1, NCHANL)

IF (NP.GT.1) WRITE II3,2390) (YPII),FPII),I =1,NP I

IF (NH.GT.1) WRITE (I3,2400) (YHII),FHII),I=1,NH)

IF IKII.EO.0.0R.KII.GT.2) GO TO 1560

If ING.LE.II GO TO $1590^{\circ}$

IF (K10.EO.1) WRITE (I3,2440) (YGII),FG(I),III,NG)

IF (KIO.EO.O) WRITE (I3,2450) (YGIII,FGII),I=1,NG)

779300

779500

779700

779800

780300

780400

780500

780600

780.700

780800

780900

781400

781500

781700

721800

781900

782200

782600

782700

782800

782800

782900

783000

783100 
1580 IF (NG.6T.1) WRITE $(13,2410)$ (YG(I),FG(I),I=1,NG)

783200

783300

783400

783500 IF (NHX.GT,1) WRITE $(I 3,2430)$ (YHXII), FHXIII, I=1, NHX

C. CARD GROUP 13

1595 IFI.NOT.PRINTI1311 GO TO 1600

PRINT $1954,1 I, I=1, N F T)$

PRINT 1961, (RZIII, I I I, NFTI

PRINT 1961, (R2III,I I I, NFT)

PRINT 1969, IRRIII, I $=1$, YF TI

PRINT 1963, HRIB

PRINT 1953, BIOTC

WRITE(13,1965) CR (3), CR (1), CR 12), (CRI1), I=4,8)

WRITEII3,1968)CGII),CGI2), (CGIII,I $=4,7)$

DC $1599 I=1$.NFT

$R 2(I)=R 2 I I 1 / 1000$.

RZSIIIERZSIII/1000.

1599. RR II)=RR II /1000

HPIB $=$ HR I B / 1000 .

1600 CONTINUE

C

RETURN

1740 FORMAT 123 HOHEAT FLUX CISTRIBUTION/23H X/L * RELATIVE FLUX

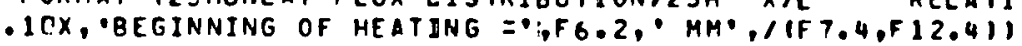

1741 FORMATI OCIRCUMFERENTIAL CONDUCTION CONSIOERED FOR ROD NUMBERS, 1, $.126151)$

1742 FORMATI ORAOIATION INCLUOEO IN HEAT FLUX CALCULATIONS'I

1 Q10 FORMAT 122 HOSUBCHANNEL INPUT DATA/IO3H CHANNEL TYPE AREA HET - TED HYDRAULIC IADJACENT CHAMNEL NOD SPACING CENTROID

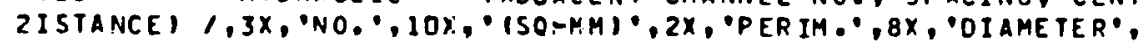

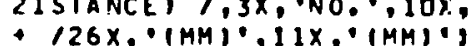

1811 FORMAT 1 $5,17,2 F 9,2, F 16,3,5 X, 411 H 113,1 H, F 5,3,1 H, F 5,3,1 H 111$

1830 FORMAT 136 HOSUBCHAYNEL THERMAL CONNECTION DATA $/ 4 X_{1}, 46$ GCONNECTION 786200 +WALL AVERAGE WIOTH OF CHANNEL, 3BH CONDUCIIVE CHANNEL CON 796300 -DUCTIVE /4X,84H NO. HEAT CAPACITY CONNECTION NO. 786400 -RESISTANCE NO. RESISTLNCE $198 X, 73 H(K J / 42-K)$ $+$ $(M 2-K / K W)$ $(42-K / K) 1$

783600

783800

783900

784100

784800

785100 785200 785300

\section{FORMAT $1110,216 \times, F B, 21,4 X, 2115,6 \times, F 9,4,3 \times 11$}

1840 FORMAT IISHOROD INPUT DATA/96H ROD DIA RAOIAL POUER

-FRACTION OF POWER TO ADJACENT CHANNELS IAOJ. CHANNEL NO.) /30H 786900 - NO. (MM) FACTOR

1841 FORMAT $115,5 X, F 7,3, F, 10,7,2 X, 71 F 8,4,1 H 1,13,1 H 111$

1850 FORMAT I32HOGAP ORIENTATION IGAP NO. ANGLEI/IBX,811HI,I3,1H,F5.0,2 787200 +H 111

1860 FORMAT 123 HOCALCULATION PARAMETERS/30H LATERAL RESISTANCE FACTOR - ,F9.4.31X,27HCHANNEL LENGTH ,F9.2.7H MM , /3OH - sili Papameter

15/2) PARAMETER
+ I9, /3OH TURBULENT MDMENTUM FACTOR, ,F9.4

1861 FORMAT 11H+.69X,27HAXIAL NODE LENGTH

1862 FORMAT ITOX, 27 HROUGHENING BEGINS

$\because, F 9,1,7 H$ MM $, 1,70 \times, 27$ HROUGHENING ENDS

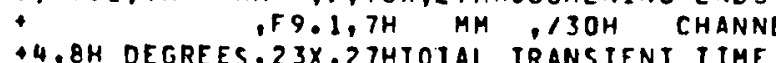

$, F 9.4,7 H \quad M M \quad$,

787300

787400

- 7OX, 27 HNUMEER OF TIME SIEPS

7OX,27HNUMEER OF TIME STEPS

1870 FORMAT 127 E.9.4,9H SECONDSI 788400 
- MIT I9,31X,27HMINIMUM INTERNAL ITERATIONS,19,130H INTERNAL IT 788800 -ERATION LIMIT ,19,31X,27HFRACTION DONOR CELL USTAR ,F9.4.130H - CONVERGENCE FACTOES $40 X, 21 H A C C E L E R A T I O N$ FACTORS U3OH - ExTERnal (OH/WI ,F9.4,31X,27H CROSSFLOH SOLUTION ,F9.4,130H INTERNAL (ONIH) ,F9.4,31X,27H LATERAL

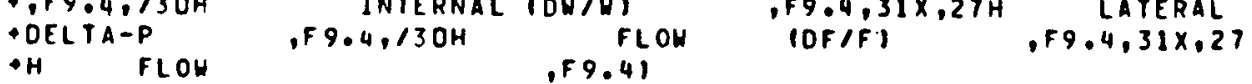

1880 FORMAT I5OHO $X / L$ AREA VARIATION FACTORS FOR SUBCHANNEL $1 I 1 / 8 X, 1$ $.012 \times, 1, .13,0, .1 \times 1)$

1890 FORMAT 16940 X $/ L$ GAP SPACING VARIATION FACTORS FOR AOJACENT SUB + CHANNELS $(1, J 1 / B X, 1011 \times, \cdot 1,12, \cdot, 12,1,11$

1900 FORMAT! OPERATING CONDITIONS, $13 x_{0}$ 'SYSTEM PRESSURE = ,FB. 2 ,

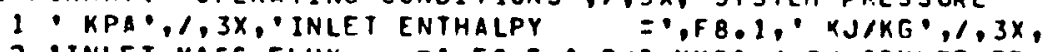
2 'INLET MASS FLUX = $, F 8,5,0$ G/5-MHZ, $/, 3 \times$, 'INLET TEMPERATURE =

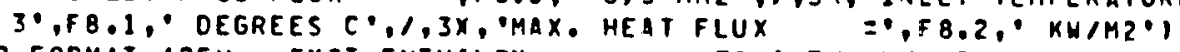

1910 FORHAT (25H EXIT ENTHALPY $=$ F8.1.74 KJIKG)

1919 FORMAT $1 / 29 H$ FRICTION FACTOR CORRELAIION $1,22 X, 24 H S H O O T H$ SECTION, .57X, 13 HROUGH SECTIONI

1920 FORMAT I/29H PRICTION FACTOR CORRELATION I

1921 FORMATIOAXIAL NODE LOCATIONS (MMI., , 1.1OFIB.11)

1928 FORMAT IIH+,94X,35H R-FUNCTION CORRELATION

1929 FORMATII4H CHANNEL TYPE, 13,8H FRICT $=, E 10,5,6 \mathrm{H} * \mathrm{RE} \$ 1, \mathrm{E} 10.4,4 \mathrm{HI}$ , E 10.5,4X,12HCHANNEL TYPE,I3,8H FRICT=,E10,5,6H\$RE\$ $\$ 1, E 10,4,4 \mathrm{HI}$. - E10.51

1930 FORMAT 126 H CHANNEL TYPE, 13,11H FRICT =,FB.3,6H\#RE\$1,F6.3,4 (H) $\mathrm{HI} 6.41$

1940 FORMATI/4X, OCHANNEL TYPE', I3,5X, 'LAMINAR FRICTION CORRELATION',4X,

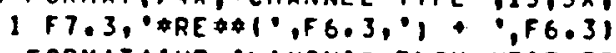

1941 FORMATIIHO, 'LAMINAR FLON HEAT TRANSFER CORRELATION, $14 X$, - CEntral channels : nu = alpha/ (1.ALPHI) $114.12: 4.13$ alog .10 .951 ALPHA-111,.14X.

- NALL/CORNER CHANNELS: NU $=4.07+1.24$, ALPHA.00.8030',

1947 FORMATI CHANNEL TYPE 1-1.11. STN = PETLKHOV-ROIZEN METHOD. $120 \times,{ }^{\circ}$ CHANNEL TYPE 1-1, II, ' STN = G-FUNCTICN CORRELATIONPI

1948 FORMAT 114H CHANNEL TYPE, 13,30H STN = PETUHHOV-ROIZEN METHOD, $22 X$,

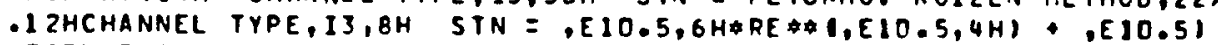
1949 FORMAT IIH+,94X,35H G-FUNCTION CORRELATION

1950 FORMAT 1/42H OITTUS-BOELTER HEAT TRANSFER CCRRELATION /6X,9H HFILM

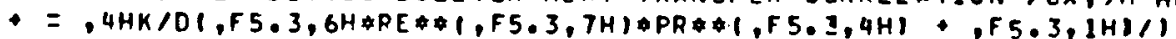

1951 FORMATI/,27H STANT ON NUMBER CORRELATION, $1,22 X, 14 H S M O O T H$ SECTION. .57X, 13 HROUGH SECTIONI

1952 FORMAT114H CHANNEL TYPE, 13,8H STN =, F10,5,6H*RE* $1, E 10,4,4 \mathrm{H})$. $\because$ E10.5,4X,12HCHANNEL TYPE, I3,8H STN = E E 10.5,6H:RE*1,E10.4,4HI". - E10.51

1953 FORMAT 1 OB 10 T COEFFICIENT, 013.3 )

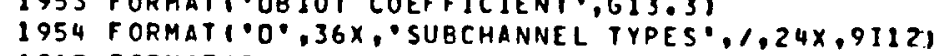

1960 FORMAT $128 \mathrm{H}$ WP $=, F 7.4,9 \mathrm{H}$ \#AP*GAVG)

1961 FORMAT ('CTUBE RAOIUS (MM)',9X,9F12.31

1962 FORMATI OORADIUS OF ZERO SHEAR IMMI:.9F12.31

1963 FORMAT I OROUGHNESS RIB HEIGHT $=0, F 133^{\circ} \mathrm{MM}$.,

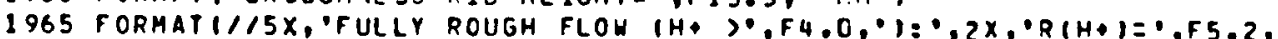

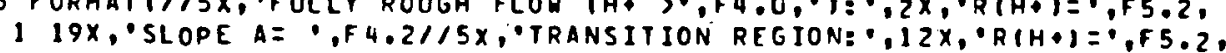

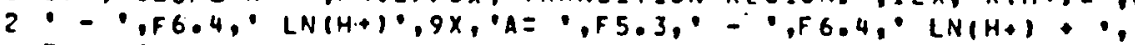

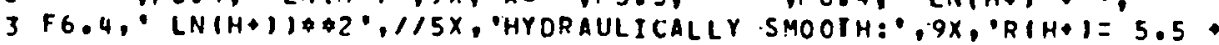
42.5 LN $1 H B+1,12 \times, \cdot A=2.50 \div, 111$

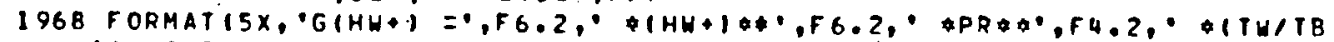

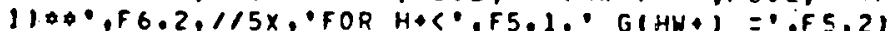

738900 738900 789000 789100 789200 789300

789800 789900

.

790700 790800 790900 791100 791200 791200 791300 791400 791500
791600 
1969 FORMAT ('OROD RADIUS (MH) , 10X, 9F 12.3)

1970 FORMAT 128H

$U P=, F 7.4,6 H \$ R E \$ 1,56.4,10 H 1 \$ 6 A P \quad 794000$ * GAVG

$W P=, F 7.4,6 H \# R E \#(, F 6.4,11 H) . D A V \quad 794200$

FORMAT 128H

794200
794300

. G*GAVG

1990 FORMAT I2OHOMIXING CORRELATIONS!

2020 FORMAT IF7.4,10F8.4)

2030 FORMAT $128 \mathrm{H}$

- LIENGTH*DAVG GAVG

794400
794800

794900

2031 FORMAT128H

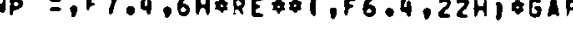

795000 II

WP $=, F 7.4,20 H * D A V_{G} * G A V G * S O R T / F / 8$

795100
795200

2230 FORMAT $142 \mathrm{H}$ CONOUCTION MIXING, GEOMETRY FACTOR = F6.41 797400

2270 FORHAT I/62H WIRE WRAP SPACER DATA FOR FORCED OIVERSION CROSSFLOH 797500 -MIXING $1 / 20 \mathrm{H}$ WRAP PITCH =F6.1.7H MM $120 \mathrm{H}$ WRAP THICKN 797600 - ESS =F6.2,7H MM $120 \mathrm{H}$ PIN DIAMETER $=56.2,7 \mathrm{H}$ MH 111

2280 FORMAT $123 \mathrm{H}$ HRAP CROSSING DATA $160 \mathrm{H}$ GAP SUBCHANNEL MI 797800 - XING RELATIVE LOCATION /6OH NO PAIR NO. PARAM 797900

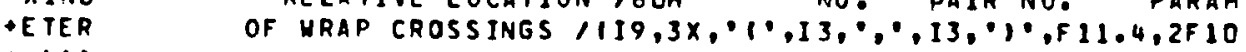

4 (1)

2290 FORMAT I/12H SPACER DATA/20H. SPACER TYPE NO.,1217

2300 FORMAT (2IH LOCATION (X/L) DI IFT.3)

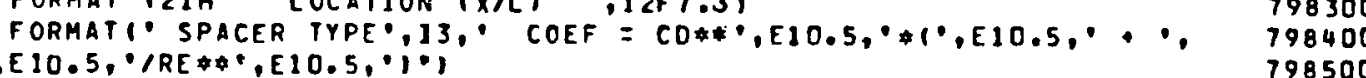

2302 FORMATI'OSPACER LOSS CQEFFICIENT', 11

2303 FORMAT IOSPACER YIOTH $=-1,7.2$, MM.

- heat tRanSFER COEFFICIENT ENHANCEMENT DUE TO SPACERS $, 1,5 x$,

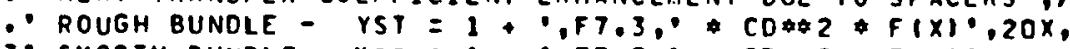

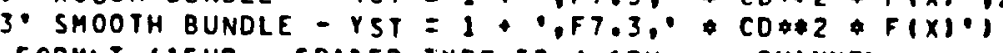

2310 FORHIT 115 HO SPAEER TYPE,12,1,60H CHANNEL CHANNEL

- Ditr nO. SOLIOITY NO. SOLIDITY,f,13X,4116,F9.4)11)

2320 FORMAT 146H INIIIAL NRAP INVENTORY FOR EACH SUBCHANMEL 11101511 799300 2330 FORMAT 143 HO FLOH DIYERSION FACTORS FOR 5PACEP IYPE 1215X46HGAP 799400

- CHANNEL FRACTION GAP CHANNEL FRACTION/5X46HNO. PAIR DIVER 759500

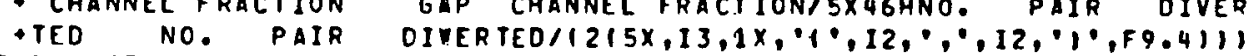

2340 FORMATIIHO. FUEL DATA:'1, ZONAL RAOII :,5F10.31

235 FORMATIIH, CONOUCTIVITY $\because 5 F 10.31$

2355 FORMATIIH: GAP CONDUCTANCE, $6 \times, 4 F 10.31$

2360 FORMATIIH: SPECIFIC HEAT $\because 5 F 10.31$

2365 FORMATIIH: POWER FRACTION: 5 F10.3)

2390 FORMAT 133 HO FORCING EUNCTION FOR PRESSURE $123 \mathrm{H}$ TIME PRE 800000 -SSURE/23H ISECI FACTOR IFIO.4,F13.4II TIME PRE 800800

2400 FORMAT 138 HO FORCING FUNCTION FOR INLET ENTHALPY/28H TIME I 801000 +NLET ENTHALP /23H ISECI FACTOR / (F10.4,F13.41) 2410 FORAAT 138 HO FORCING FUNCIION FOR INLET FLON $128 \mathrm{H}$ TIME 1 801200

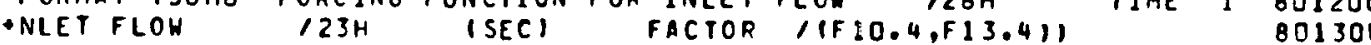
2420 FORMAT I38HO FORCING FUNCTION FOR HEAT FLUX /38H TIME BDIUOO +HEAT FLUX I23H ISEC) FACTOR/F10.4.F13.41 391500 i 2430 FORMAT 138 HO FORCING FUNCTION FOR EXIT ENTHALPY $138 \mathrm{H}$ IIME 801600 - ENTHALPY I24H ISECI FACTOR /1F10.4,F13.1 8D1800 $+11$

2440 FORMAT 145 HO FORCING FUNCTION FOR PRESSURE DIFFERENCE 122K 802000 2450 TIME OELTA-PI2IH ISECI FACTORIIFIO.4,F13.411 83.100 - TIME DELIA-P/2OH (SEC) (PSII/(FID.4.F13.4)) 
2460 FORMAT 130 HO UNIFORM INLET ENTHALPY

802400

2470 FORMAT 135 HO UNIFORM INLET TEMPERATURE

1

802500

2480 FORMAT 165 HO INOIVIDUAL SUBCHANNEL ENTHALPY SPECIFIEO ICHANNEL

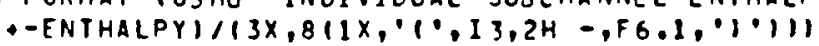

2490 FORMAT 171 HO INDIVIOUAL SUBCHANNEL TEMPERATURE SPECIFIED. ICHAN

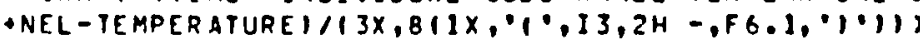

802600

2500 FORMAT $135 \mathrm{HO}$ UVIFORM INLET HASS VELOCITY I

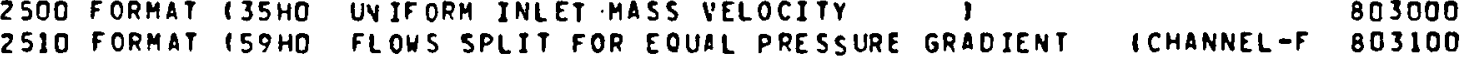

2520 FORMAT 157 THO INDIVIOUAL SUBCHANNEL FLOUS SFECIFIED ICHANMEL-FLO

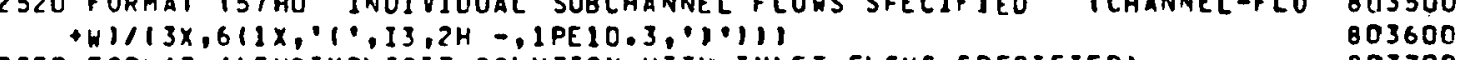

2550 FORMAT 145HOIMPLICIT SOLUTION WITH INLET FLCHS SPECIFIEDS

2620 FORMAT ( OHELIUM PROPERTIES AT',FB.2,4H KPAI

2622 FORMATI1OX,1HT, 8X,1HH,
$10 \times 2 H C P, 12 \times 1 H V, 12 \times 4 H V 1 S C$

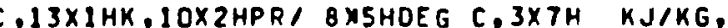

805400

2630 FORMAT $16 x, F 7,1,3 x, F 7,1,5 x, F 6,3,7 x, F 7,4,5 x, F, 10,7,6 x, F 9,6,6 x, F 5.31$ END

aHOG

- S P B A

2PRT, 5

COBRA $\triangle C F$ TL SPBFA

FURPUR 2ER1 GA-B E35 S74T11 05/26/81 17:37:09

0
1
0 


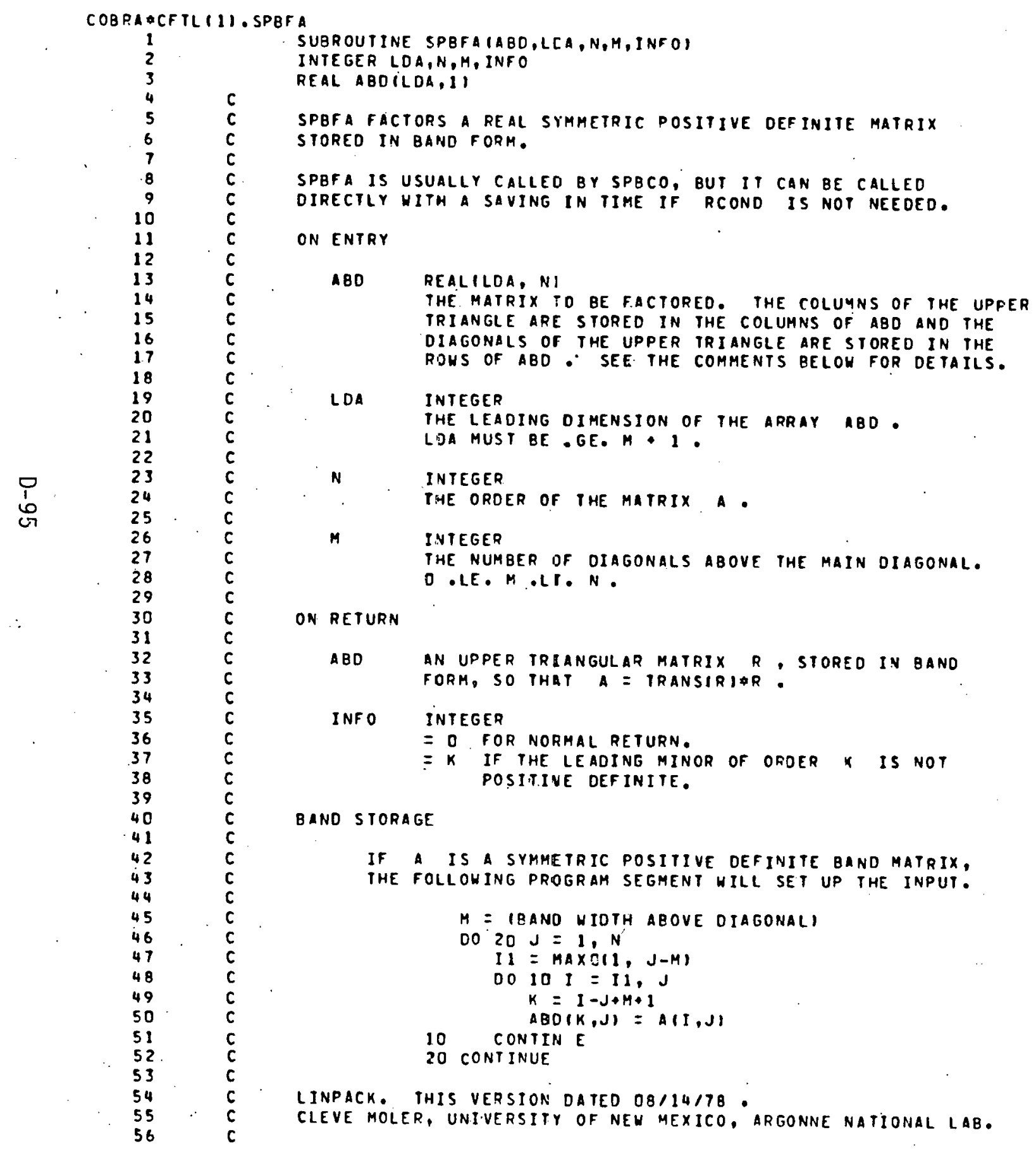




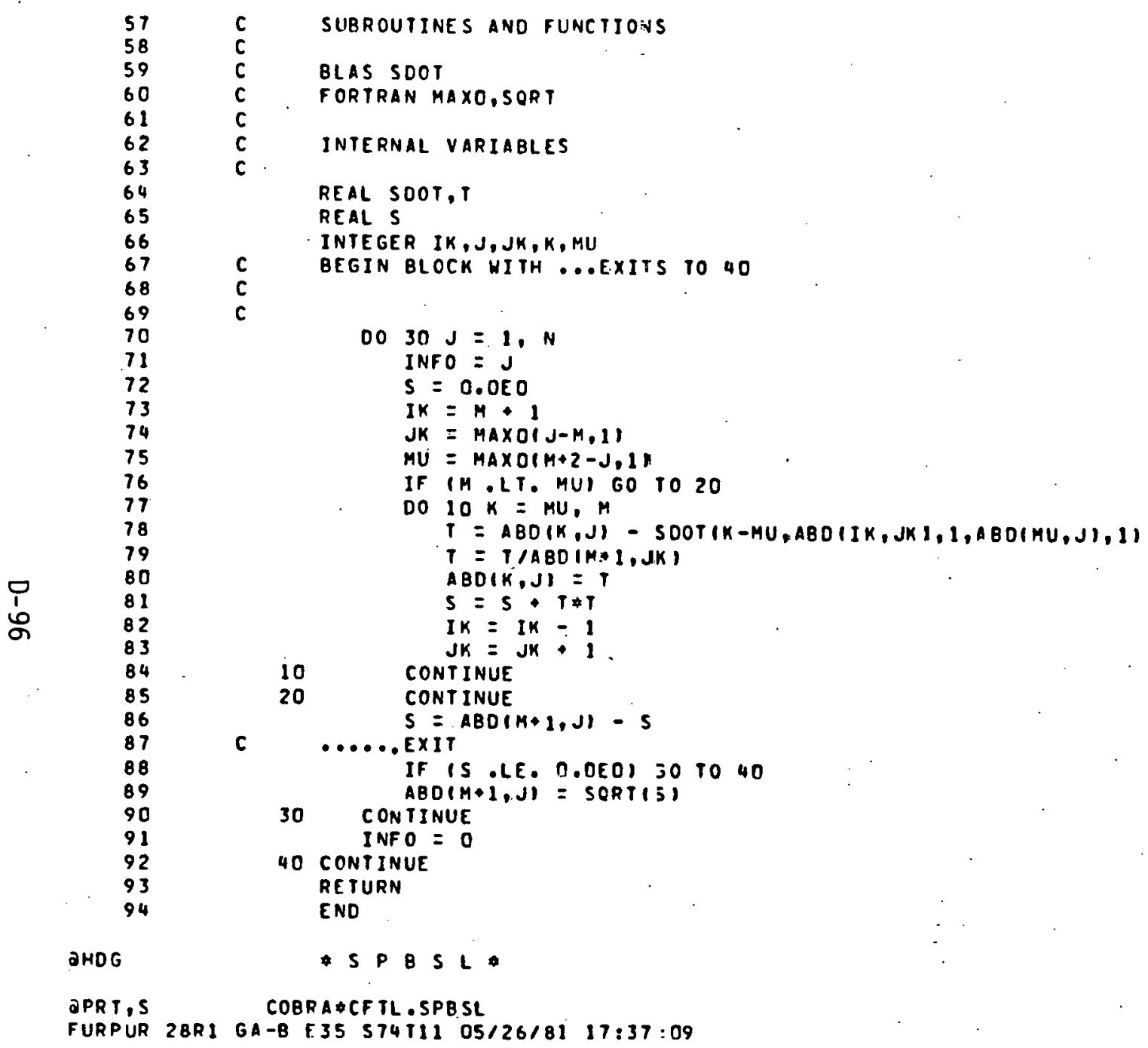


COBRAACFIL (1).SPESL

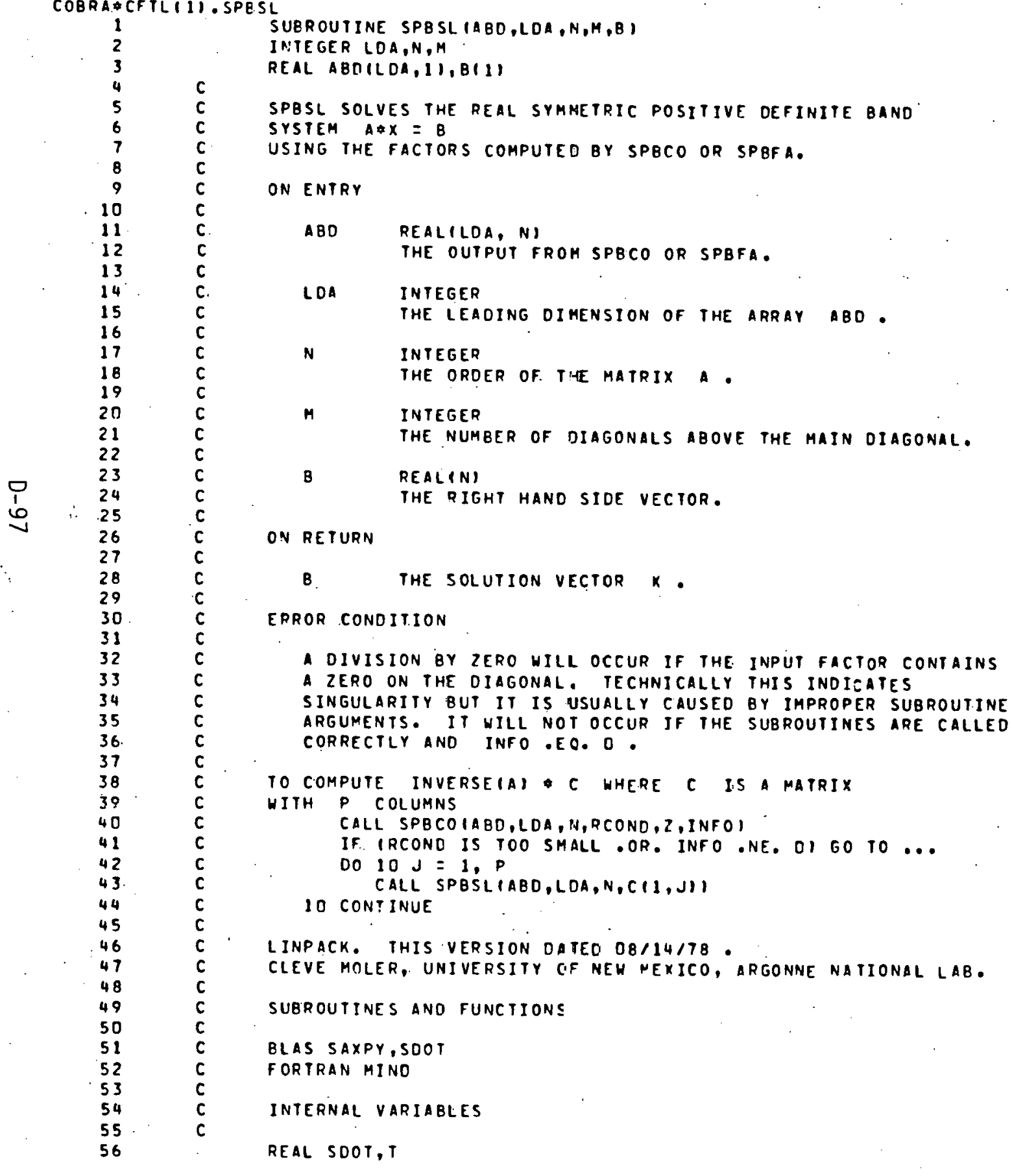


- SPESL*

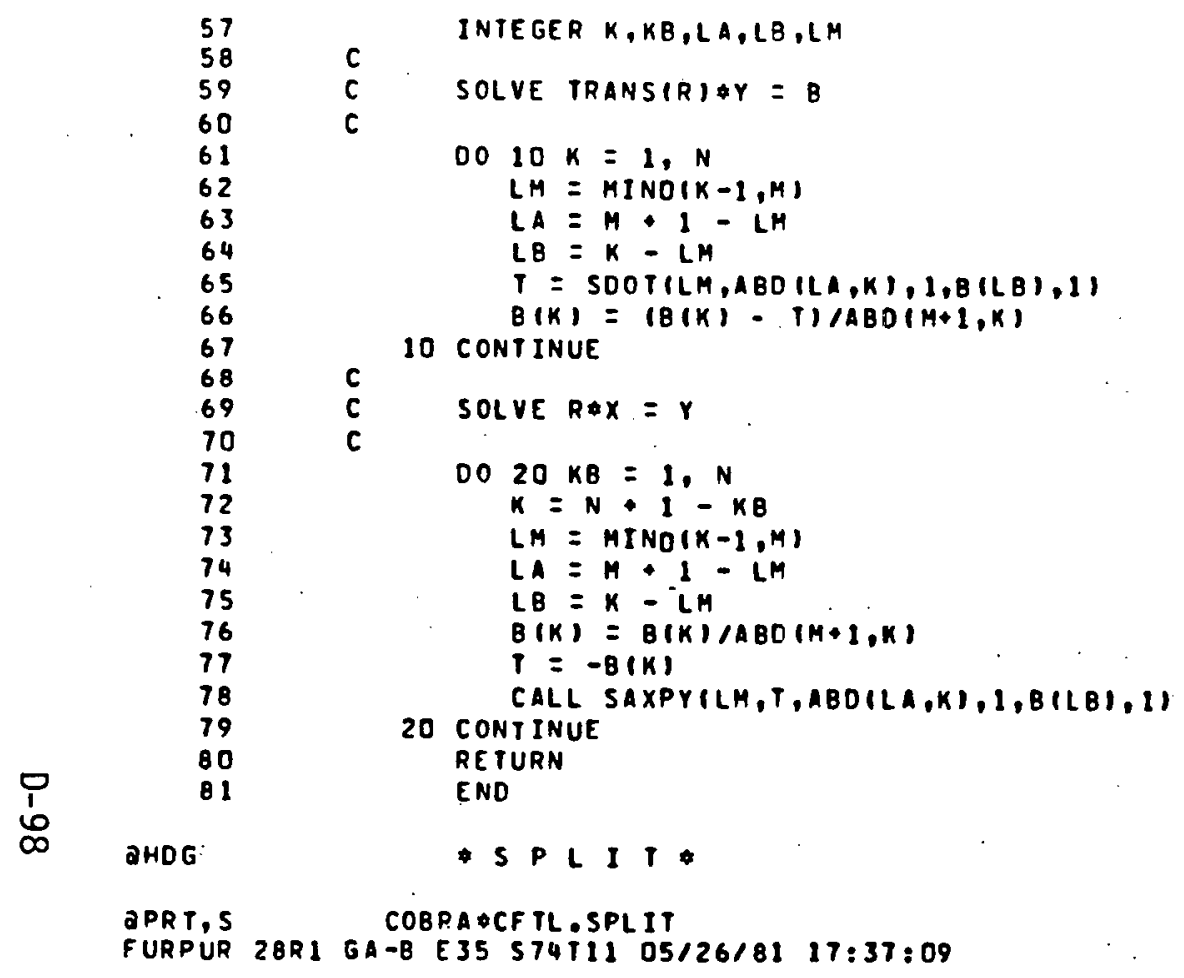


- SPLII

CCBRAOCTIL1.1).SPLIT

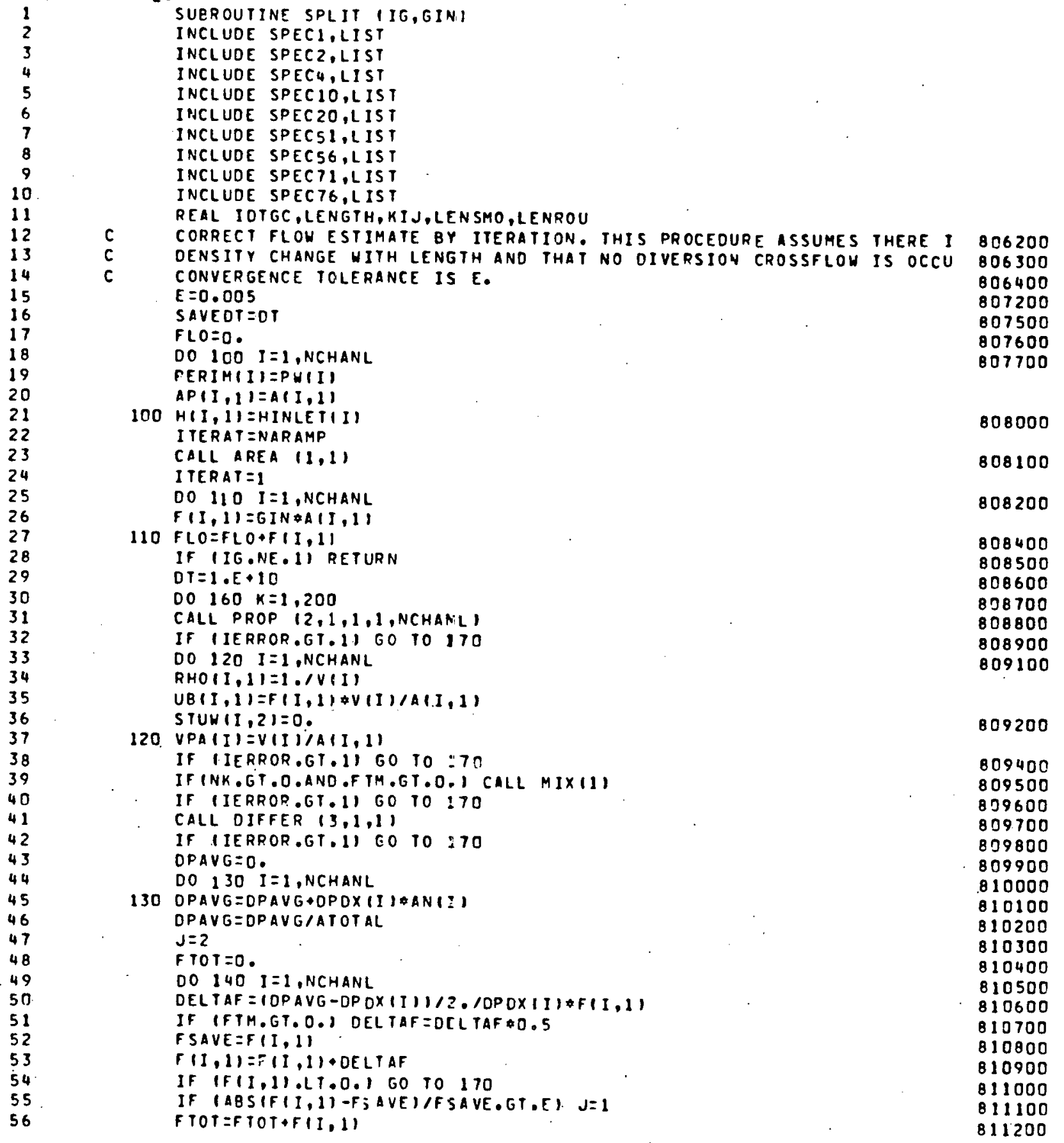


* SPLIT:

140 CONTINUE

OO $150 \quad I=1$, NCHANL

811300

811400

811500

SO FINLETIII, II FLOIFTOT

IF (J.GT.I) GO 0180

160 CONTINUE

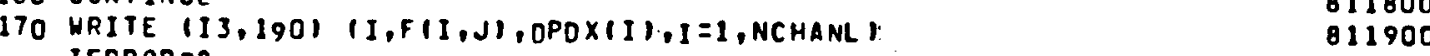

811500

11700

811700

$\begin{array}{ll}\text { IERROR }=8 & 812000\end{array}$

180 DTISSAVEOT

812000
812100

RETURN

812200

812300

812400

190. FORMAT 14OH FLOW S.PLIT TO GIVE EOUAL DP/OX FAILED /115,2E14.611

812400 END

812600

AHOG

* I E R O I T.

COBRA*CF TL - ZEROIT

FURPUR 28R1 GA-B E 35 $574 T 11$ 05/26/81 17:37:10 
COBRA *CFTL 11 . ZEROIT

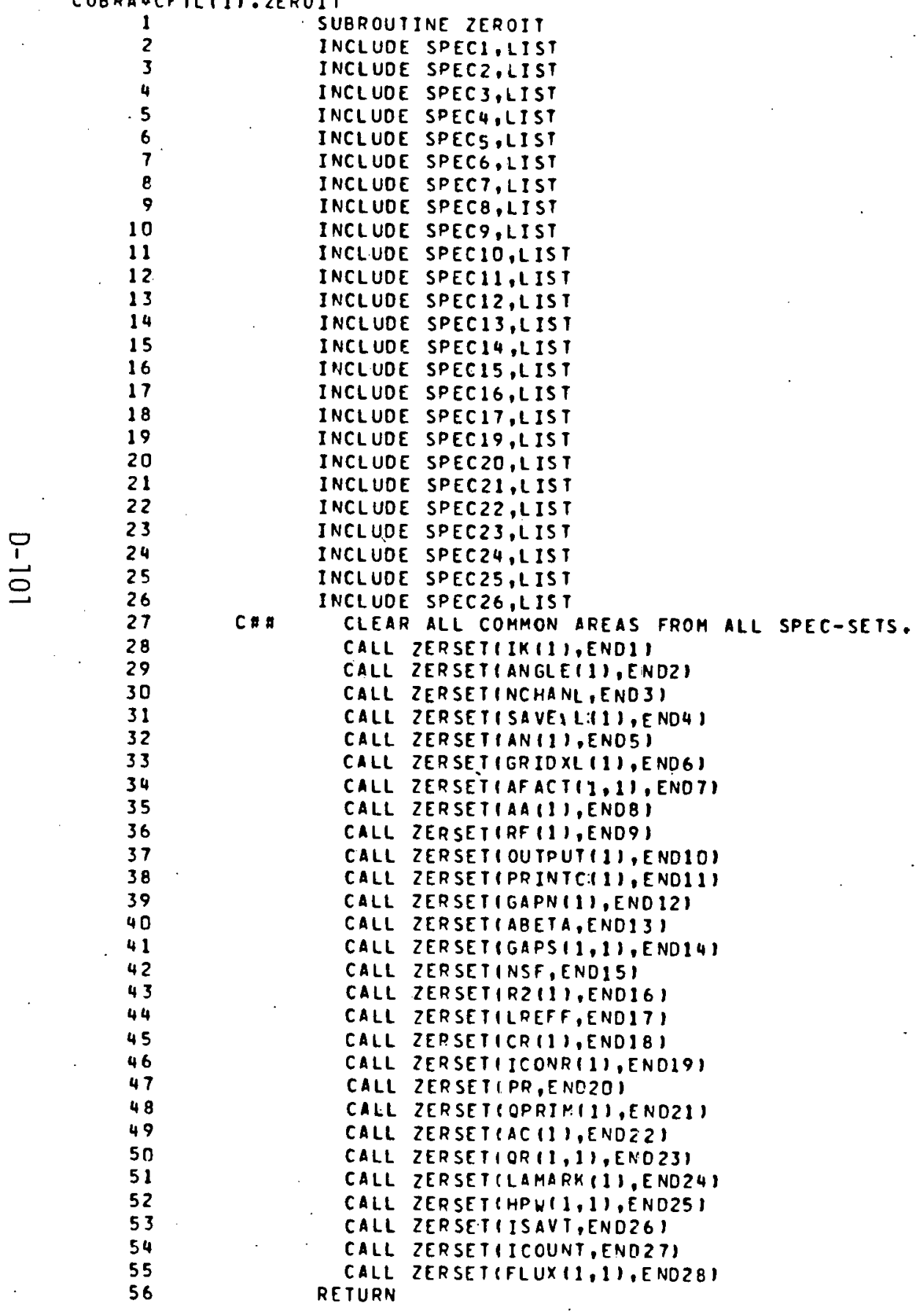


COBRAOCFILI11. ZERSET

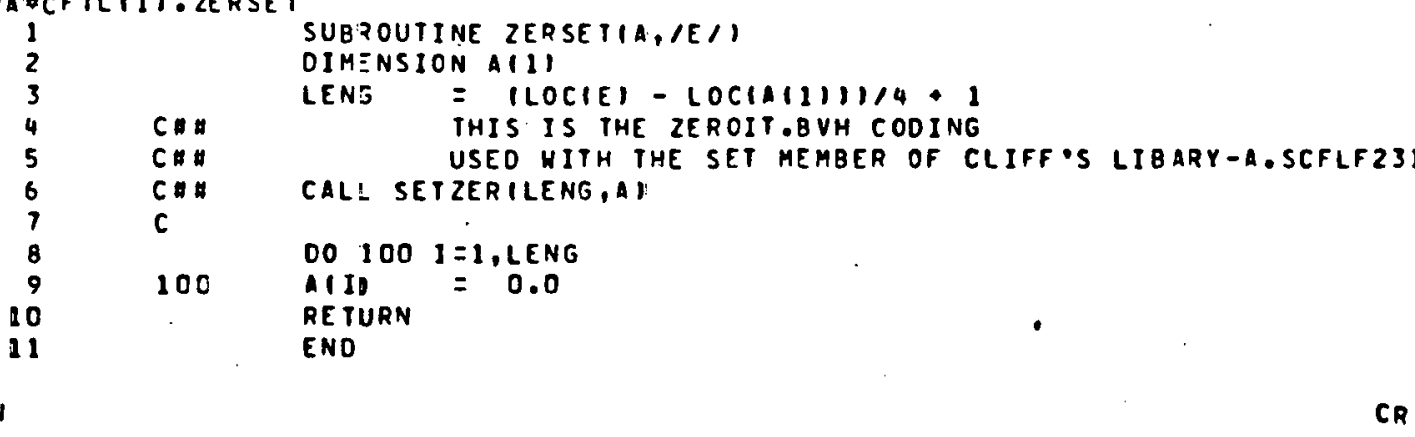


ORNL/SUB-81/92304/1

GA-A16359

Dist. Category UC -77

INTERNAL DISTRIBUTION

1. R. L. Anderson

2. S. J. Ball

3. N. C. J. Chen

4. W. R. Clark

5. C. W. Collins

6. J. C. Conklin

7. R. L. Cox

8. U. Gat

9. G. E. Giles

10. A. G. Grindell

11. P. G. Herndon

12. S. A. Hodge

13. F. J. Homan
14-22. P. R. Kasten

23. R. E. MacPherson

24. R. W. McCulloch

25. T. W. Robinson, Jr.

26-35. J. P. Sanders

36. H. E. Trammell

37. H. C. Young

38. ORNL Patent Office

39. Central Research Library

40. Document Reference Section

41-42. Laboratory Records Department

43. Laboratory Records, RC

\section{EXTERNAL DISTRIBUTION}

44-45. Director, Office of Nuclear Power Systems, DOE, Washington, D.C. 20545.

46. G. A. Newby, Director, High-Temperature Reactor Division, ONPS, DOE, Washington, D.C. 20545

47. L. M. Welshans, High-Temperature Reactor Division, ONPS, DOE, Washington, D.C. 20545

48. J. P. Radcliffe, Office of Program Management, Research and Space Programs, D0E, P. 0. Box 81325, San Diego, Calif. 92138

49. Manager, San Francisco Operations Office, DOE, 1333 Broadway, Wells Fargo Building, Oakland, Cal if. 94162

50. Office of Assistant Manager for Energy Research and Development, Oak Ridge Operations Office, DOE, Oak Ridge, Tenn. 37830

51. Joseph Pidkowicz, Oak Ridge Operations Office, DOE, Oak Ridge, Tenn. 37830.

52. C. B. Baxi, General Atomic Company, P. 0. Box 81608, San Diego, Calif. 92138

53-217. Given distribution as shown in DOE/TIC-4500 under category UC-77 (Gas-Cooled Reactor Technology:) 